\title{
Localized Wave Pulse Experiments
}

\author{
D. Kent Lewis \\ David H. Chambers \\ Kenneth L. Krueger \\ Terry L. Henderson \\ Richard W. Ziolkowski
}

June 1999

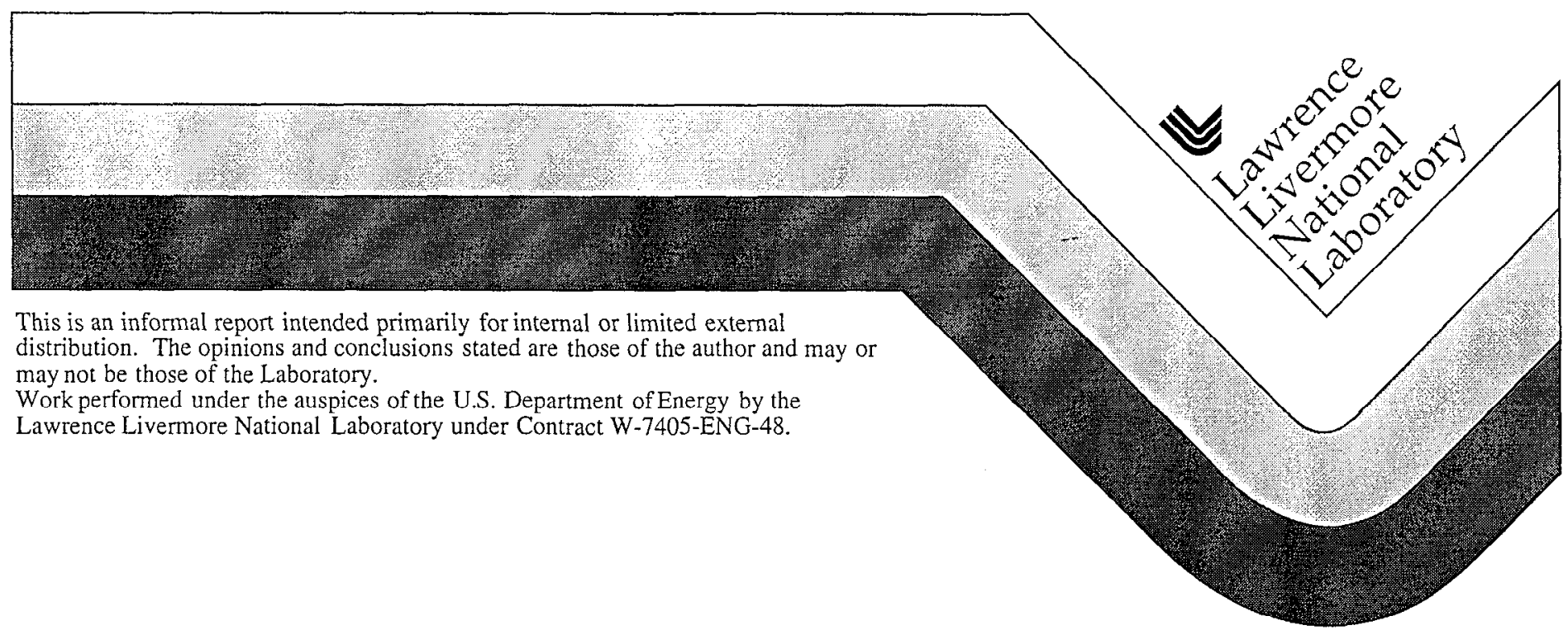




\section{DISCLAIMER}

This document was prepared as an account of work sponsored by an agency of the United States Government. Neither the United States Government nor the University of California nor any of their employees, makes any warranty, express or implied, or assumes any legal liability or responsibility for the accuracy, completeness, or usefulness of any information, apparatus, product, or process disclosed, or represents that its use would not infringe privately owned rights. Reference herein to any specific commercial product, process, or service by trade name, trademark, manufacturer, or otherwise, does not necessarily constitute or imply its endorsement, recommendation, or favoring by the United States Government or the University of California. The views and opinions of authors expressed herein do not necessarily state or reflect those of the United States Government or the University of California, and shall not be used for advertising or product endorsement purposes.

This report has been reproduced directly from the best available copy.

Available to DOE and DOE contractors from the Office of Scientific and Technical Information

P.O. Box 62, Oak Ridge, TN 37831

Prices available from (615) 576-8401, FTS 626-8401

Available to the public from the

National Technical Information Service

U.S. Department of Commerce

5285 Port Royal Rd.,

Springfield, VA 22161 
Final report for the

\section{Localized Wave Pulse Experiments}

conducted at ARL Lake Travis Facility during March and April, 1998

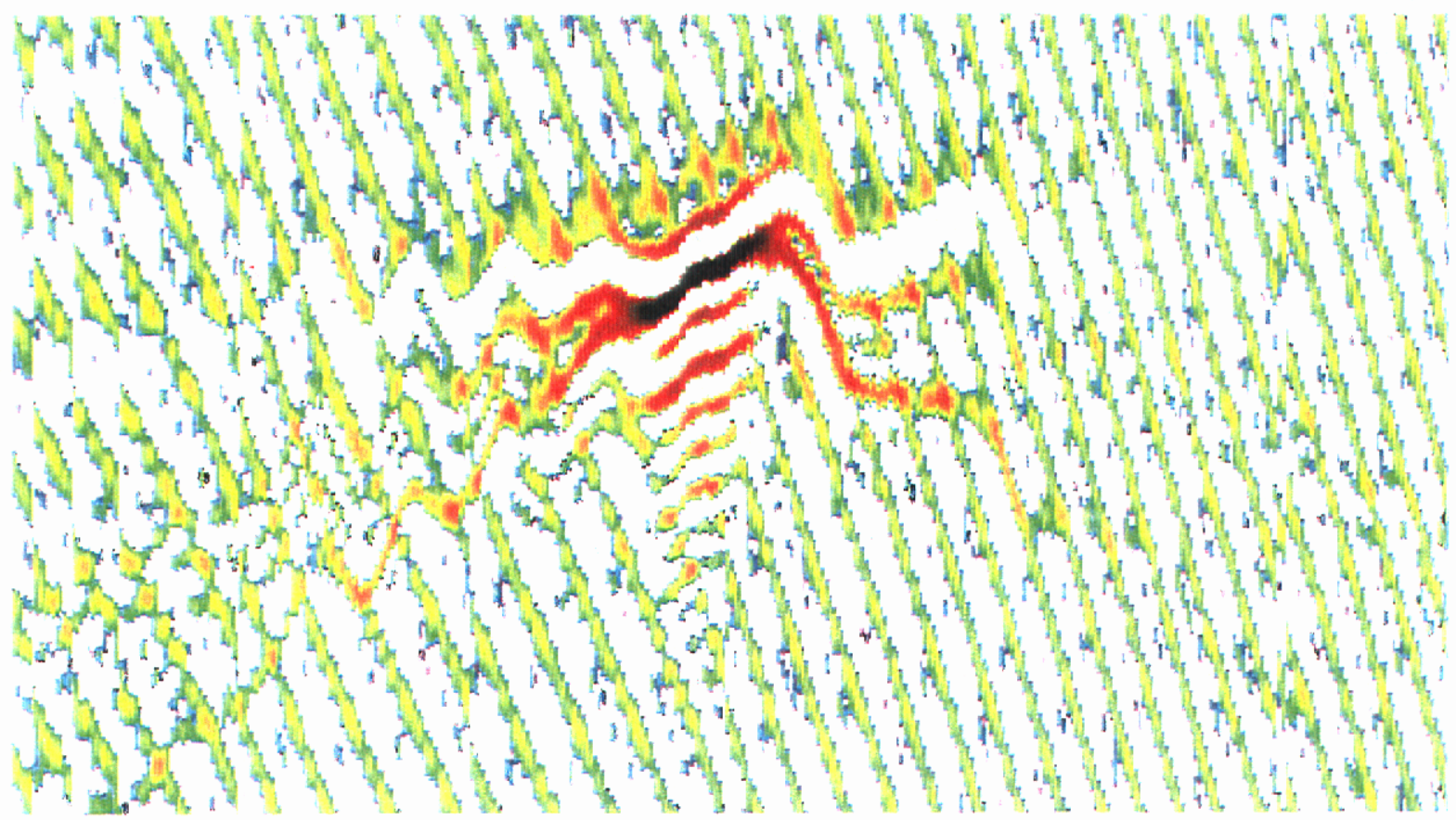

Strategic Systems Support Program

Lawrence Livermore National Laboratory

and

Applied Research Laboratory

University of Texas at Austin

D. Kent Lewis

David H. Chambers

Kenneth L. Krueger

Terry L. Henderson

Richard W. Ziolkowski

Lawrence Livermore National Laboratory

Lawrence Livermore National Laboratory

Univ. Texas: Applied Research Laboratory

Univ. Texas: Applied Research Laboratory

University of Arizona 


\section{Executive Overview:}

We proposed to

'Take the LW phenomenon from a laboratory curiosity

and prove its effectiveness as a usable field system'

Specific Goals were

(1) Produce a LW pulse beam which would maintain its form and propagate well into the far field

(2) Use a power source generating higher voltage levels $(100 \mathrm{~s}$ or $1,000 \mathrm{~s}$ of volts) than previously used (10s of volts)

Field Tests conducted

(1) Field experiments were conducted at the ARL:UT facility at Lake Travis using a 24 ring array designed for the experiment The drive channels used 10 s of volts

(2) LLNL designed the source signals and analyzed the data, Pulse launch and data acquisition were done by ARL:UT

Results:

(1) Localized Wave pulses were created and propagated in open water, through a thermocline, well past the near field transition point

(2) The LW pulses had very narrow beam width, extremely wide frequency band width, and no observable side lobes, as predicted

(3) Pulse pressures and beam widths were measured at 100' and 600'

(4) LW performance was better than all off-resonance tone bursts, and out-performed the equivalent piston pulses 
The Localized Wave project of the Strategic System Support Program has recently finished an experiment in cooperation with the Advanced SONAR group of the Applied Research Laboratory of the University of Texas at Austin

The purpose of the experiment was three-fold. We wanted to see if (1) the LW pulse could propagate over significant distances, to see if (2) a new type of array and drive system specifically designed for the pulse would increase efficiency over single frequency tone bursts, and to see if (3) the complexity of our 24 channel drivers resulted in better efficiency than a single equivalent pulse driving a piston. In our experiment, several LW pulses were launched from the Lake Travis facility and propagated over distances of either 100 feet or 600 feet, through a thermocline for the 600 foot measurements.

Our results show conclusively that the Localized Wave will propagate past the near field distance. The LW pulses resulted in extremely broad frequency band width pulses with narrow spatial beam patterns and unmeasurable side lobes. Their array gain was better than most tone bursts and further, were better than their equivalent piston pulses.

This marks the first test of several Low Diffraction beams against their equivalent piston pulses, as well as the first propagation of $\mathrm{LW}$ pulses over appreciable distances. curiosity.

The LW pulse is now proven a useful tool in open water, rather than a laboratory

The experimental system and array were built by ARL, and the experiments were conducted by ARL staff on their standard test range. The 600 feet measurements were made at the farthest extent of that range.

\section{Objectives:}

The purpose of the Lake Travis experiments was three-fold. We were to demonstrate that the Localized Wave beam would propagate into the far field, specifically that it would propagate over the 200 yard range normally used for long range measurements at Lake Travis. We were also to compare the beam profile, frequency content and array gain of a Localized Wave beam to 5 cycle single frequency tone bursts over the range of useful frequencies of the array. We were also to compare the LW pulses to their piston equivalents.

We demonstrated that the Localized Wave beam would propagate to the end of the Lake Travis range by detecting 3 different pulses at a distance of 600 feet. We will use these pulses in the following comparisons. These pulses were the Superposed Gaussian 1 (SG1) pulse, the Modified Pulse Spectrum 1 pulse (MPS1), and the Modified Pulse Spectrum 3 (MPS3) pulse. The following data compares beam profile and array gain between the $\mathrm{LW}$ pulses, the $20 \mathrm{kHz}$ and $100 \mathrm{kHz}$ tonebursts, and the $\mathrm{LW}$ piston equivalent. Results of these tests are shown in figures Objectives-1, 2, and 3.

We also showed that among the 3 Localized Waves used, an array of different beam widths and array gains could be achieved. This is the adaptive nature of the beam which we have discussed. To be able to design an optimum beam would mean that we knew all the characteristics of the array, receiver, and electronics, characteristics we could not measure in the Lake Travis experiments.

One of the things we were interested in was a search for side lobes. The theory predicts no side lobes at all, but our ring based simulator predicted that there would be some structure below $50 \mathrm{~dB}$ down from the maximum due to a grating effect of the evenly spaced rings. Unfortunately, the data only achieved slightly more than $30 \mathrm{~dB}$ due to the low source voltages used in the measurements. The input voltage was turned down for the 600 foot data relative to the 100 foot case (see Table 1 in this report). This comparison is shown graphically in Figure 25 of the main body of the report.

The Localized Wave piston equivalent was formed by driving all of the array elements with the signal designed for the array center. The reason for this was that this is the drive signal which results if we build a signal set for a single driver with the same size and frequency bandwidth as the 24 ring array. These piston signals have a frequency 
distribution (Figure Objectives -4) which encloses all of the other 23 drive signals. This suggests that driving a piston with a broad frequency band width can still be done optimally using Localized Wave design tools. To achieve additional array gain, an individually addressable array must be used.

We proved that the LW pulse propagates into the far field, maintaining shape and frequency band width. We showed that the LW pulse, with a decade of band width (comparison in Figure Objectives-5), can achieve array gains comparable to single frequency beams, and that the LW pulse outperformed its piston equivalent in all our tests.

We were unable to confirm the theoretical prediction that $L W$ pulses beams generate no side lobes. Further tests at higher power, or repeatable tests in a more controlled environment could determine this.

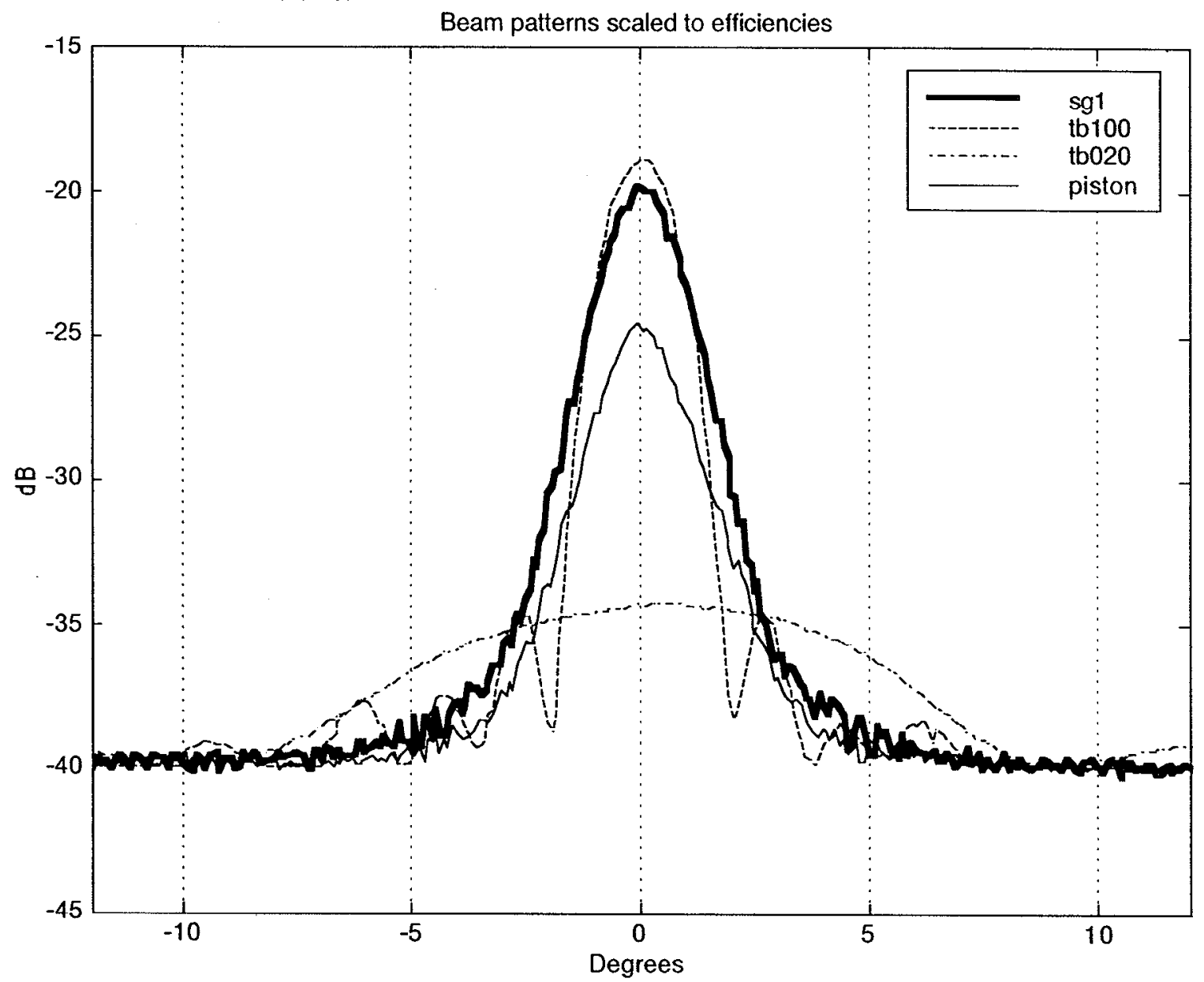

Figure Objectives-1. The normalized beam patterns for the Localized Wave pulse (Superposed Gaussian 1) with a frequency bandwidth spanning the range from $10 \mathrm{kHz}$ to $250 \mathrm{kHz}$. The 4 curves have been normalized so that the efficiencies of the beams are shown as well as the beam profiles by dividing the received energy as a function of angle by the input energy used to create the beam. All have been normalized to the same noise floor. The four beams (and their raw data representation locations) are SG1, the Localized Wave pulse (Figure E1-23), the $100 \mathrm{kHz}$ toneburst TB100 (Figure E1-28), the SG1 equivalent piston (Figure E1-24), and the $20 \mathrm{kHz}$ toneburst TB020 (Figure E1-26). 


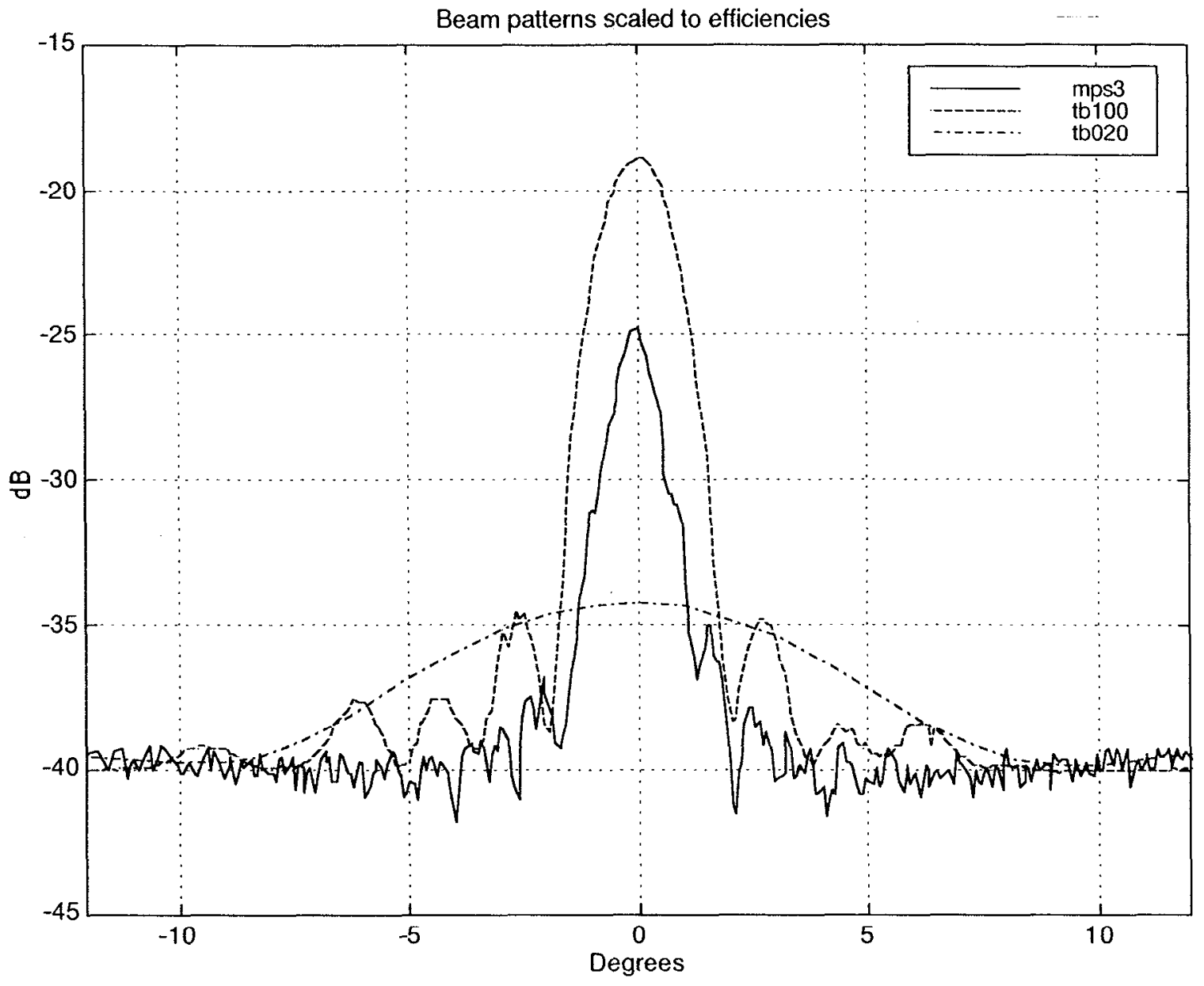

Figure Objectives -2 . The normalized beam patterns for the Localized Wave pulse (Modified Pulse Spectrum 3) with a frequency bandwidth spanning the range from $10 \mathrm{kHz}$ to $250 \mathrm{kHz}$. The 3 curves have been normalized so that the efficiencies of the beams are shown as well as the beam profiles by dividing the received energy as a function of angle by the input energy used to create the beam. All have been normalized to the same noise floor. The 3 beams (and their raw data representation locations) are MPS3, the Localized Wave pulse (Figure E1-12), the $100 \mathrm{kHz}$ toneburst TB100 (Figure E1-28), and the $20 \mathrm{kHz}$ toneburst TB020 (Figure E1-26). The piston equivalent for this case was suspected to be bad data (Figure E1-13), and so was not included. 


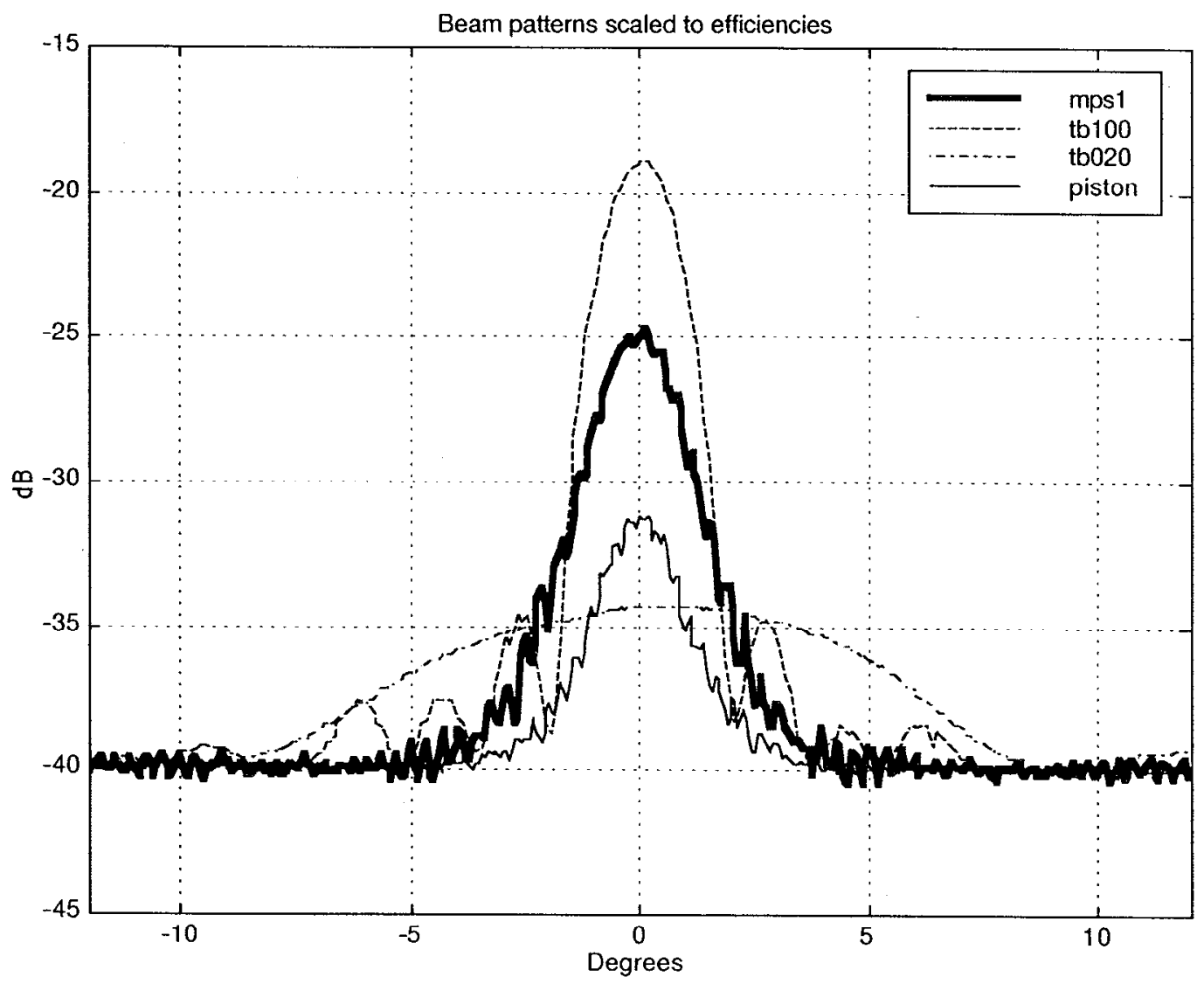

Figure Objectives -3 . The normalized beam patterns for the Localized Wave pulse (Modified Pulse Spectrum 1) with a frequency bandwidth spanning the range from $10 \mathrm{kHz}$ to $250 \mathrm{kHz}$. The 4 curves have been normalized so that the efficiencies of the beams are shown as well as the beam profiles by dividing the received energy as a function of angle by the input energy used to create the beam. All have been normalized to the same noise floor. The 4 beams (and their raw data representation locations) are MPS1, the Localized Wave pulse (Appendix E1-6), the $100 \mathrm{kHz}$ toneburst TB100 (Appendix E1-28), the piston equivalent for MPS1 (Appendix E1-7), and the $20 \mathrm{kHz}$ toneburst TB020 (Appendix E1-26). 


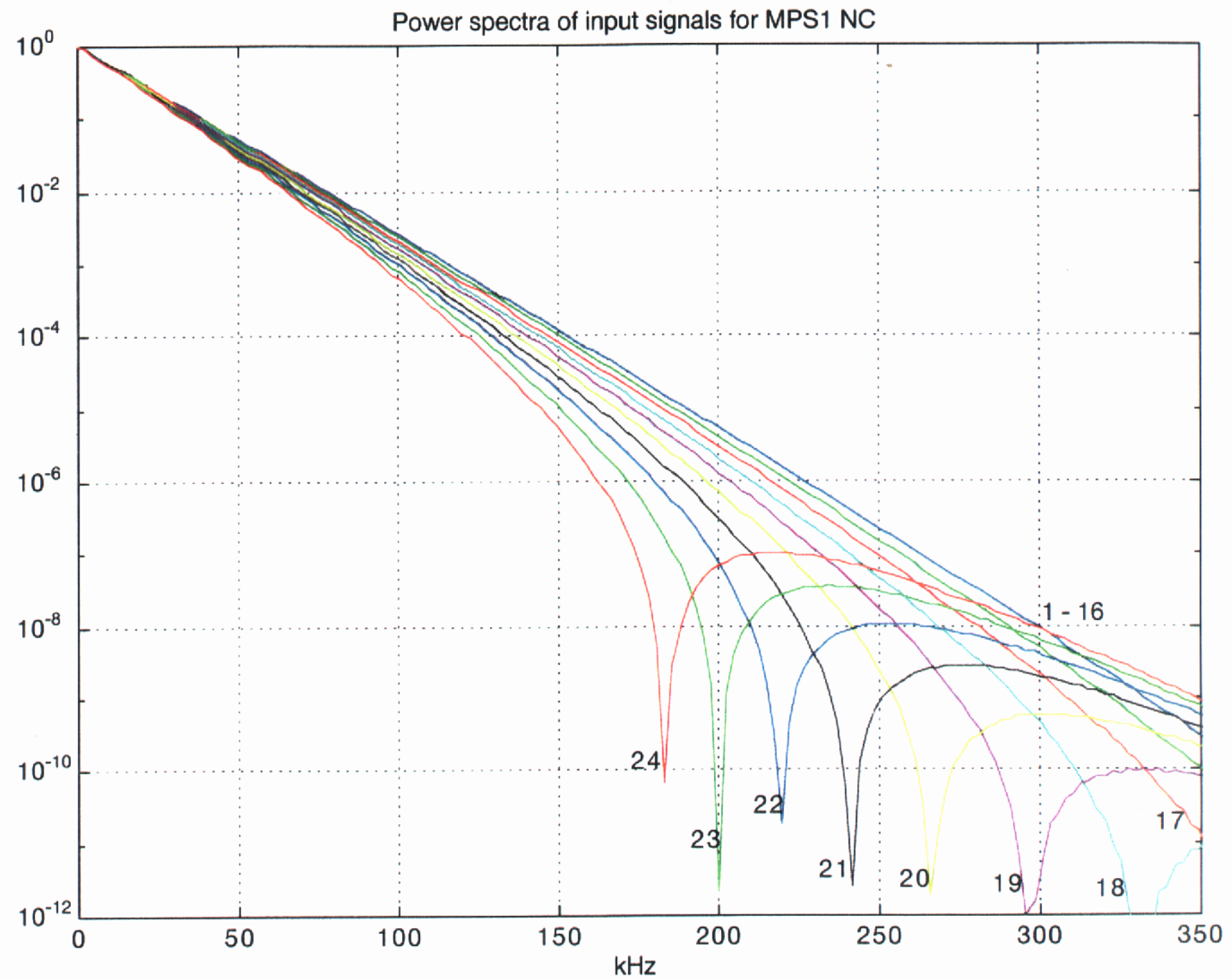

Figure Objectives -4. The input spectra for SG1 (Figure F.1.b). Each factor of 10 on the horizontal axis is $10 \mathrm{~dB}$. The SG1 equivalent piston is created by using the signal designed for the center ring, signal 1 , to power all 24 rings in the array. This signal was chosen since it envelopes all of the other signals, and because it contains the most energy in the high frequencies. The numbers in the picture coorespond to the ring numbers they are sent to, 1 (center) through 24 (outside ring). In this frequency range, rings 1 through 15 are essentially driven by the same signal for the Localized Wave pulse, and rings 1 through 24 are the same signal for the equivalent piston for SG1. 

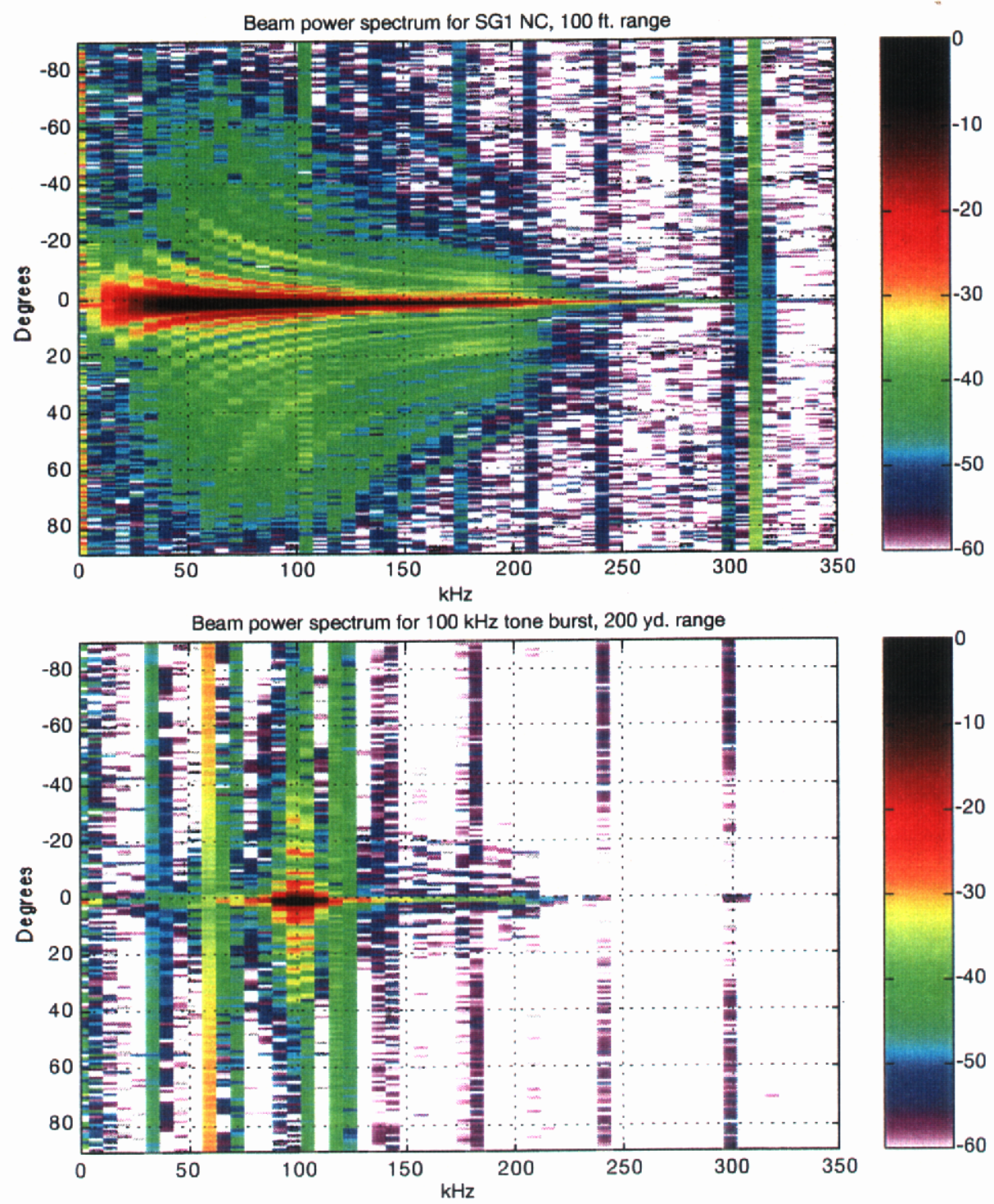

Figure Objectives -5 . The intensity as functions of beam angle and frequency for the Localized Wave pulse beam SG1, above, and the $100 \mathrm{kHz}$ tone burst, below. The on axis energy of the SG1 beam spans more than 5 octaves (for the $10 \mathrm{~dB}$ energy range) while the 5 cycles of $100 \mathrm{kHz}$ spans less than an octave. The lake noise is clear in the SG1 plot because the array driver output voltage in that case was less than for the tone burst. 
Table of Contents:

\begin{tabular}{|c|c|}
\hline i & Abstract \\
\hline ii & Objectives \\
\hline iii & Table of Contents \\
\hline I. & Introduction \\
\hline II. & $\begin{array}{l}\text { Background } \\
\text { Strategic System Support Program }\end{array}$ \\
\hline II.1 & $\begin{array}{l}\text { Strategic System Support Program } \\
\text { Applied Rescarch Laboratories of The University of Texas at Austin }\end{array}$ \\
\hline II. 2 & $\begin{array}{l}\text { Applied Research Laboratories of The University of Texas at Austin } \\
\text { Historical development of the LW concept }\end{array}$ \\
\hline II. 3 & \\
\hline II.3.1 & $\begin{array}{l}\text { Theoretical: Initial development } \\
\text { Experimental: Acousto-Optical array simulation }\end{array}$ \\
\hline II.3.2 & Experimental: Acousto-Optical array simulation \\
\hline II.3.3 & Experimental: Two transducer array simulation \\
\hline II.3.4 & Experimental: Laboratory arrays \\
\hline II.3.5 & Theoretical: Extension to the Superposed Gaussian Pulse \\
\hline II.3.6 & Experimental: NUWC Keyport experiment \\
\hline II.3.7 & Theoretical: Passive Matched Filtering \\
\hline III. & Theory \\
\hline III. 1 & System Simulation \\
\hline III. 2 & Comparison of piston to Individually Addressable Array \\
\hline IV & Experiment \\
\hline IV.1 & Equipment and System \\
\hline IV.2 & System Characterization \\
\hline IV.3 & Experimental Method \\
\hline IV.3.1 & Pulse Stretching for Noise Reduction \\
\hline IV.3.2 & Environmental Effects \\
\hline IV.3.3 & Pulse Choice for Comparison \\
\hline V & Data Analysis \\
\hline V.1 & Amplitude Correction \\
\hline V.2 & System Compensation \\
\hline V.3 & Time Window Resizing \\
\hline VI & Results \\
\hline VI.1 & Comparison to Tone Bursts \\
\hline VI.1.1 & $100 \mathrm{kHz}$ Observation \\
\hline VI.1.2 & $100 \mathrm{ft}$. Data \\
\hline VI.1.3 & $600 \mathrm{ft}$. Data \\
\hline VI.1.4 & Comparison to Pulses \\
\hline VII & Tabulated Data \\
\hline VII.1 & Relative Energy \\
\hline & Table 1. Energy calculations in $\mathrm{dB}$. \\
\hline VII.2 & Beam Widths \\
\hline VIII & $\begin{array}{l}\text { Table 2. Beam Width (Full Width at Half Max.) in degrees } \\
\text { Conclusions and Recommendations }\end{array}$ \\
\hline IX & $\begin{array}{l}\text { Conclusions and Recommendations } \\
\text { Appendices }\end{array}$ \\
\hline IX.1 & Appendix A: Frequency Dependent Behavior of the System \\
\hline IX.2 & Appendix B: System Diagram \\
\hline IX.3 & Appendix C: Source Voltage and Current Analysis \\
\hline IX.4 & Appendix D: Received Signal Spectra \\
\hline IX.5 & Appendix E: Beam Patterns \\
\hline IX.5.1 & Angular Patterns \\
\hline IX.5.2 & Polar Patterns \\
\hline IX.6 & Appendix F: Source Signals \\
\hline IX.7 & Appendix G: Test Plan \\
\hline
\end{tabular}




\section{Introduction}

The Localized Wave pulse is a broad frequency band width pulse built by the linear superposition of well characterized basis functions, either the Focus Wave Modes of Brittingham as developed by Ziolkowski, or the Gaussian Pulse as developed by Chambers. The beam results in lower side lobes and a more narrow width than other beams through constructive interference on axis and destructive interference off axis.

LW pulses can be described by the linear superposition of infinite plane waves, as can any linear beam, but the first beam was first made by superposing the FWM wave solutions using the Modified Pulse Spectrum of Ziolkowski. The idea behind this was, and is, that superposing solutions that do what one wants is a simpler way to a beam than casting about in Fourier space. In 1996 a second LW pulse was designed using Gaussia individually optimized for each array ring. The two pulses are designated the MPS and SG pulses respectively.

The Localized Wave project has been designing pulses for both acoustic and microwave arrays since 1981. There has been an active experimental effort since 1986, and a total of 6 acoustic proof of principle experiments have now been conducted, while the first microwave experiment is planned for 1998 .

The first two experiments were simulations of radiation from arrays, as described in the Background section. The following 4 experiments used individually addressable arrays with $6,7,11$ and 24 channels, and have launched LW pulses into a variety of media, including fresh, deionized water, lake water, and sea water.

\section{Background}

\section{II.1 Strategic System Support Program}

The Localized Wave project is part of the Strategic System Support Program. This program operates under the Defense and Nuclear Technologies Directorate of Lawrence Liverinore National Laboratory.

The Strategic Systems Support Program excels in assembling multi-disciplinary scientific teams to solve problems related to the "Science of Seawater". To that end we have established both an acoustic and a microwave project for Low Diffraction Beam rescarch and development. We have established a working relationship and participated in experiments with the Navy Underwater Weapons Center, Penn. State's Applied Physics Laboratory, Northrop Grumman, University of Arizona's clectronics and microwave department, and now the University of Texas.

\section{II.2 Applied Research Laboratories of The University of Texas at Austin}

The Applied Research Laboratories of The University of Texas at Austin (ARL:UT) conducts programs of basic and applied research, development, engineering, testing, evaluation, and assessment, primarily for the United States Department of Defense. ARL:UT evolved from two defense-oriented laboratories established by The University during World War II. It performs those functions appropriate for a university laboratory - teaching, research, and public service.

Although recent trends have greatly increased the proportion of work performed for the other services, the Navy has been the primary customer historically, and includes ARL:UT in its organizational chart as one of 6 university laboratories, along with the Applied Research Laboratory of Pennsylvania State University, the Applied Physics Laboratory of the University of Washington, the Applied Physics Laboratory of the Johns Hopkins University, Woods Hole Oceanographic Institute, and Scripps Institute of Oceanography.

ARL:UT has a long history in the sonar community, and has developed and improved sonar systems for everything from mine hunting and obstacle avoidance to passive listening and surveillance, spanning frequencies from a few Hertz to more than a megahertz. 


\section{II.3 Historical development of the I.W concept:}

The Localized Wave work described in this brief overview is a part of the overall work on Low Diffraction beams in both acoustics and microwave research and development. Other workers in the acoustics field are Jim Greenleaf, with the X wave beam, and Peter Stepanishen, with the Acoustic Bessel Bullet.

The Localized Wave predates both of the above by a few years, and the following description of the steps in its development are purposefully brief. Further information is available from the references, or from the authors.

\section{II.3.1 Theoretical: Initial development}

The LW work began with the work of Jim Brittingham (1) at Livermore in the '70s. He worked for 11 years to find a transverse solution to Maxwell's equations, and finally found wave solutions which he called Focus Wave Modes. These modes travel at the wave speed and never change in any way. Like infinite plane waves, they require an infinite amount of energy to create and an infinite aperture to launch, which makes both waves impossible to create. But beams can be made out of either which are physically possible.

Rick Ziolkowski looked at these wave equation solutions as building blocks, and proved that they comprised a complete set of basis functions for the wave equation. He found their othoganality relationships and showed that real pulses with finite energy could be made from FWM functions by using the Modified Pulse Spectrum (2) which he worked out.

Ziolkowski's microwave simulations showed astonishingly good beam qualities, and solutions were developed for free space, wave guides, and optical fiber. All this work was in the 10s of Ghz range though, and there were no sources capable of creating the fields necessary for an experimental verification of the theory. We are currently conducting a microwave experiment in the 100s of Mhz which we hope to complete in Fall ' 98.

\section{II.3.2 Experimental: Acousto-Optical array simulation}

Lewis and Cook had been working in Non Destructive Evaluation using an arbitrary wave form generator to create a better beam for scanning. They had found a way to create virtually any pressure field from ordinary transducers and begun considering array radiators (3). They realized that they could create $\mathrm{LW}$ pulses acoustically in the $10 \mathrm{~s}$ of $\mathrm{kHz}$ range fairly simply.

The first proof of principle experiment was an acousto-optical transient measurement of the wave fields created by a single, small ( $6 \mathrm{~mm}$ diameter) transducer. By measuring the effect of the transient acoustic field travcling along the $\mathrm{z}$ axis at right angles to a steady state laser on the laser along the $\mathrm{x}$ axis, the experiment of measuring transient radiation from cylindrical transducers could be carried out. The resultant agreement between theory and experiment was extraordinary (4), and plans for a simulated axisymmetric element array were put forward.

\section{II.3.3 Experimental: Two transducer array simulation}

The second proof of principle experiment replaced the acousto-optical detector with a second transducer capable of motion in both $\mathrm{x}$ and $\mathrm{y}$. A square array of 221 elements was simulated by moving the source to a position in $x$ and $y$ at some fixed range $z$, and launching the 11 source signals as well as calibration single frequency tone bursts.

The resultant fields were then reconstructed in a computer. As expected the beam created by this axially symmetric array was better than the linear array modeled in the first experiment, and closely matched the predicted results (5).

\section{II.3.4 Experimental: Laboratory arrays}

The first actual acoustic LW pulse was launched in 1987 at LLNL. Created by a 5 by 5 square array divided into 6 groups of elements, this experiment used newly developed multi channel, programmable voltage sources exciting an individually addressable array with all the necessary source signals simultaneously.

The first array was made of a $1 \mathrm{~cm}$ square of PVDF and coated with a sputtered matrix of conductive dots in a 5 by 5 arrangement on the damped side of the transducer 
material (6). A follow-on array was 11 concentric rings on a $2 \mathrm{~cm}$ piece of PVDF (7). Both arrays were highly damped to provide a very low $Q$ and a broad frequency band width.

The detector in both cases was the same detector used in the simulated array experiments. Again the beam showed high localization of energy and no side lobes. Agreement of the experimental data to the theoretical predictions was excellent.

\section{II.3.5 Theoretical: Extension to the Superposed Gaussian Pulse}

A newly developed Low Diffraction beam was introduced by David Chambers, and is based on a superposition of single frequency Gaussian beams. The width of each single frequency basis function is chosen so that all the frequencies begin to spread spherically at the same down range point. This insures that all the wavefronts in the pulse overlay exactly for all frequencies.

The amplitude as a function of frequency is shaded by an exponential function. This function introduces another parameter allowing the beam to be tailored for any application.

\section{II.3.6 Experimental: NUWC Keyport experiment}

A joint experiment with the Navy Underwater Weapons Center at Keyport, WA was conducted using a standard Navy array. This array was stripped of all electronics, and t/- 10 volt, wide band width amplifiers were attached to each of the array's 52 elements.

Four Low diffraction pulse beams were selected and seven signals designed to drive the transducers for each case, as well as several single frequency tone bursts to check the array performance and give known benchmarks as to beam shaping ability of the array. All beam shape measurements were carried out from -90 degrees to 90 degrees off axis.

Beam shapes and on axis power versus down range distance were measured for the candidate beams in the ATATF redwood tank at Keyport. Clear evidence of the maintenance of on axis power characteristic of LW pulses was observed, and the beam shapes of the LW pulses matched predictions closely.

The transducer array was extremely resonant at certain frequencies, and refused to radiate above its upper frequency range, but pre-corrections to the source signals allowed us to create the necessary fields and launch LW pulses with excellent spectra compared to those predicted. The pre-correction necessary resulted in a lowering of the relative efficiencies of the LW beams, but the gains in lack of side lobes and 3 octaves of usable bandwidth more than offset this effect.

\section{II.3.7 Theoretical: Passive Matched Filtering}

A zeroth order study was carried out to see if the LW idea could be applied to passive arrays as successfully as it had been to active beam forming. Gaussian pulses were modeled as radiating from two different locations into a linear, vertical array in an idealized acoustic medium. An optimized Gaussian filter was applied to the array, and the signals were easily detected.

Next a LW pulse (a MPS source set) was constructed for an aperture having the same diameter as the array length, and the signals prescribed for each element location used in the matched filter. Again, the signals were easily detected at up to a $20 \mathrm{~dB}$ signal to noise ratio, but the detected signals were much more closely matched to the actual distribution of energy in both time and position than in the matched Gaussian filter, which suggested improved temporal resolution.

To test the temporal resolution of the LW filter, the sources were placed at the same relative spatial location and only slightly separated in time. While the matched Gaussian found the signals, it could not resolve them into separate components, but the LW filter could.

\section{Theory}

Three localized wave pulses were launched from the 24 ring array at Lake Travis. Two of these were Modified Power Spectrum (MPS) pulses (derived by Ziolkowski ${ }^{2}$ ), and 
one was a Superposed Gaussian (SG) pulse which is a new type of localized wave derived by Chambers. Each LW pulse is specified by parameters unique to each type. In this section the theory behind the generation of the specific voltage wave forms is explained, including the selection of LW pulse parameters.

The first step is to assume that the LW analytical expressions represent the acoustic potential $\Phi(r, z, t)$ in the aperture of the array. The velocities of the element heads are given by the derivative of the potential sampled at the element locations $\left(x_{n}, y_{n}\right)$ :

$$
u_{n}(t)=u_{z 0}\left(r_{n}, t\right)=\left.\frac{\partial \Phi}{\partial z}\right|_{z=0}, \quad r_{n}=\sqrt{x_{n}^{2}+y_{n}^{2}} .
$$

Ideally the voltage applied to each element is the time integral of the velocity desired for that element. However, the actual voltage waveform is the convolution of the ideal voltage with the inverse filter derived from the calibration runs. This compensates for the limitations imposed by the actual hardware in the frequency band between $10 \mathrm{kHz}$ and $280 \mathrm{kHz}$. Outside of this frequency band the elements did not radiate any appreciable energy.

The potential for the MPS pulse is given by 1,2 :

$$
\begin{aligned}
& \Phi(r, z, t)=\operatorname{Re}\left[\frac{2 R_{m} z_{0}}{z_{0}+i(z-c t)} \frac{\exp \left(-z_{0} s(r, z, t) / w_{0}^{2}\right)}{s(r, z, t)+2 R_{m}}\right], \\
& s(r, z, t)=\frac{r^{2}}{z_{0}+i(z-c t)}-i(z+c t) .
\end{aligned}
$$

The parameters $z_{0}, w_{0}$, and $R_{m}$ completely specify the MPS pulse properties. In previous experiments the range parameter $R_{m}$ was chosen to be large. Here it was set to 1000 meters. The MPS pulse is insensitive to the exact value of $R_{m}$ as long as its large. The other two parameters are the main means of controlling the MPS pulse properties.

The effective frequency is obtained from the Fourier transformed driving voltages:

$$
f_{e f f}^{2} \equiv \frac{\sum_{n} f^{2} \hat{V}_{n}(f)}{\sum_{n} \hat{V}_{n}(f)},
$$

where $\hat{V}_{n}(f)$ is the Fourier transform of the nth element driving voltage.

The other LW pulse was the Superposed Gaussian (SG). This were originally formulated to provide a comparison to the MPS pulse. As originally formulated by Ziolkowski, the MPS pulse is a superposition of Brittingham's Focused Wave Modes 1 . Following this example the Superposed Gaussian pulses are superpositions of Gaussian Beam Modes. The fundamental Gaussian beam for a given wave number $\mathrm{k}(=\omega / c)$ is ${ }^{2}$

$$
u_{k}(r, z, t)=\frac{k w_{0}^{2} \exp (i(\omega t-k z))}{k w_{0}^{2}-2 i\left(z-z_{0}\right)} \exp \left(-\frac{k r^{2}}{k w_{0}^{2}-2 i\left(z-z_{0}\right)}\right),
$$

where $z_{0}$ is the focal plane position, and $w_{0}$ is the beam waist in the focal plane. A superposition of Gaussian beams of different frequencies can be written as 


$$
\Phi(r, z, t)=\int_{0}^{\infty} \hat{\Phi}(\omega) \frac{\omega w_{0}^{2} \exp (i \omega(t-z / c))}{\omega w_{0}^{2}-2 i c\left(z-z_{0}\right)} \exp \left(-\frac{\omega r^{2}}{\omega w_{0}^{2}-2 i c\left(z-z_{0}\right)}\right) d \omega,
$$

where $\hat{\Phi}(\omega)$ is the weighting for the beam with angular frequency $\omega$. In order to obtain a simple analytical form the beam waist is chosen to be a function of frequency, $w_{0}(\omega)=r_{0} \sqrt{\omega_{0} / \omega}$, and the weighting is chosen to be $\hat{\Phi}(\omega)=\exp \left(\omega / \omega_{0}\right) / \omega_{0}$. With these choices the integral can be performed giving the final expression for the acoustic potential of the SG pulse:

$$
\Phi(r, z, t)=\operatorname{Re}\left[\frac{1}{r^{2} / r_{0}^{2}+\left(1-i \omega_{0}(t-z / c)\right)\left(1-2 i c\left(z-z_{0}\right) / \omega_{0} r_{0}^{2}\right)}\right],
$$

The parameters $\mathrm{r}_{0}$ (transverse halfwidth), $\mathrm{z}_{0}$, and $\omega_{0}\left(=2 \pi f_{0}\right)$, completely specify the SG pulse.

\section{III.1 System Simulation}

To design signals for the test, we built a simulator based on the work of Stepanishen (8). This code was built in MatLab, and tested against the Gaussian pulse result cited in the paper as a test of accuracy.

The simulator is driven by entering the first time derivative of the source voltages as the sound velocity, and the final result, the pressure time signal, is then differentiated again to yield the voltage measured at the receiver hydrophone. Our simulator then measures the voltage at the detector digitizer resulting from the input of a voltage pulse at the source.

\section{III.2 Comparison of piston to Individually Addressable Array Single Frequency Case:}

In the experimental sections of this report, we will address the effects of using only the temporal filter characteristics of the Localized Wave pulse when we use the LW pulse characteristic signal to drive a piston, which has no spatial variability at all. But first we examine in simulation the case of ignoring the temporal filtering effects, and concentrate only on the spatial filtering used by the MPS pulse. This will not create a LW pulse, since that depends on using the inseparable space-time filter, but may give us insight into better ways of generating a single frequency tone burst with a segmented array.

What we will do is as ordinary as focusing a single frequency tone burst at a certain axial distance would be, as an example, but focusing is only possible over a limited range down axis. We consider the case of beam formation in the very far field, hundreds of diameter down range, well past the range that focusing could have any effect.

Consider the case of constructing a single frequency beam using the Localized Wave technique. In this case we'll use the Superposed Gaussian-1 pulse, which has an effective frequency of $46.8 \mathrm{kHz}$, with no compensation and only look at the contributions to the field at $97.66 \mathrm{kHz}$, a close enough approximation to $100 \mathrm{kHz}$. Using the simulator to reconstruct the field amplitudes at this frequency from each of the 24 rings individually, we see that there are side lobes present for each of the rings, but that they are not summing constructively except along the axis.

These different beam patterns were calculated by simulating the launch of an MPS pulse from an array consisting of a single ring of varying diameter and width, or by considering the case of a segmented 24 ring array with only a single driving signal fed to a single array ring for 24 cases. The resulting field line at $97.66 \mathrm{kHz}$ of the broad band width spectrum was then extracted for further analysis. This simulates a set of ring 
elements driven by a very long single frequency tone burst with the amplitude and initial phase of the driver prescribed by the SG theory.

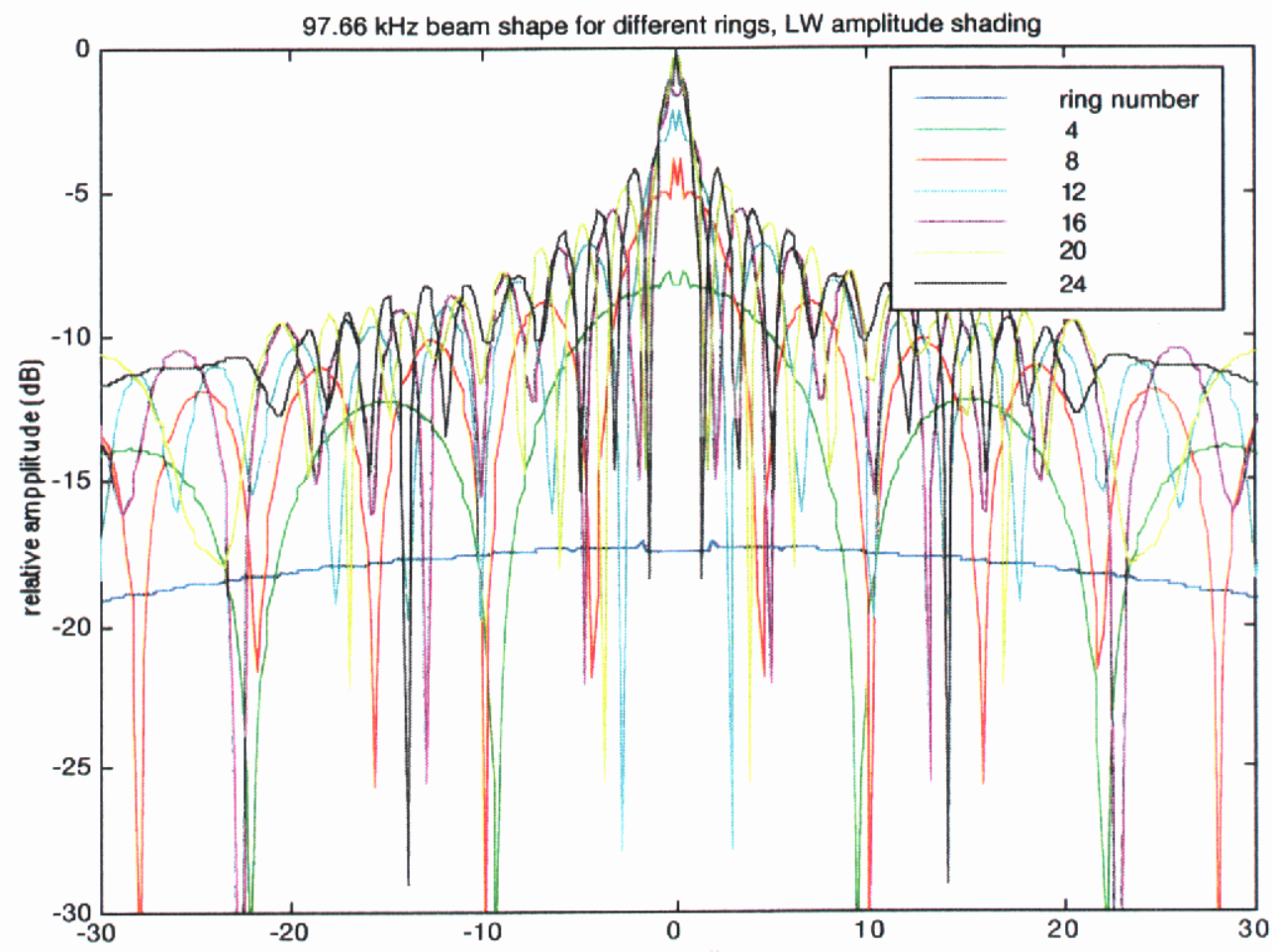

Figure 1. The field amplitudes as functions of angle for a $97.66 \mathrm{kHz}$ tone burst from some of the 24 rings of the array. The rings generate a central lobe and side lobes. The main lobes sum constructively, while the side lobes don't.

Each ring's signal has a phase associated with each angular position, phase as a function of angle, but we partially ignore that in the following analysis. When we sum all of the amplitudes with zero relative phase, which is to say use a spatial filter but not any temporal shaping, the beam can be made as compact along the axis as a piston beam, as shown below. We have achieved a localization of the single frequency tone beam, but with an elevation and smoothing of the accompanying side lobes. The full LW theory of course uses the phase that we have thrown away in the above analysis, and so achieves an even better localization of energy along the axis. 


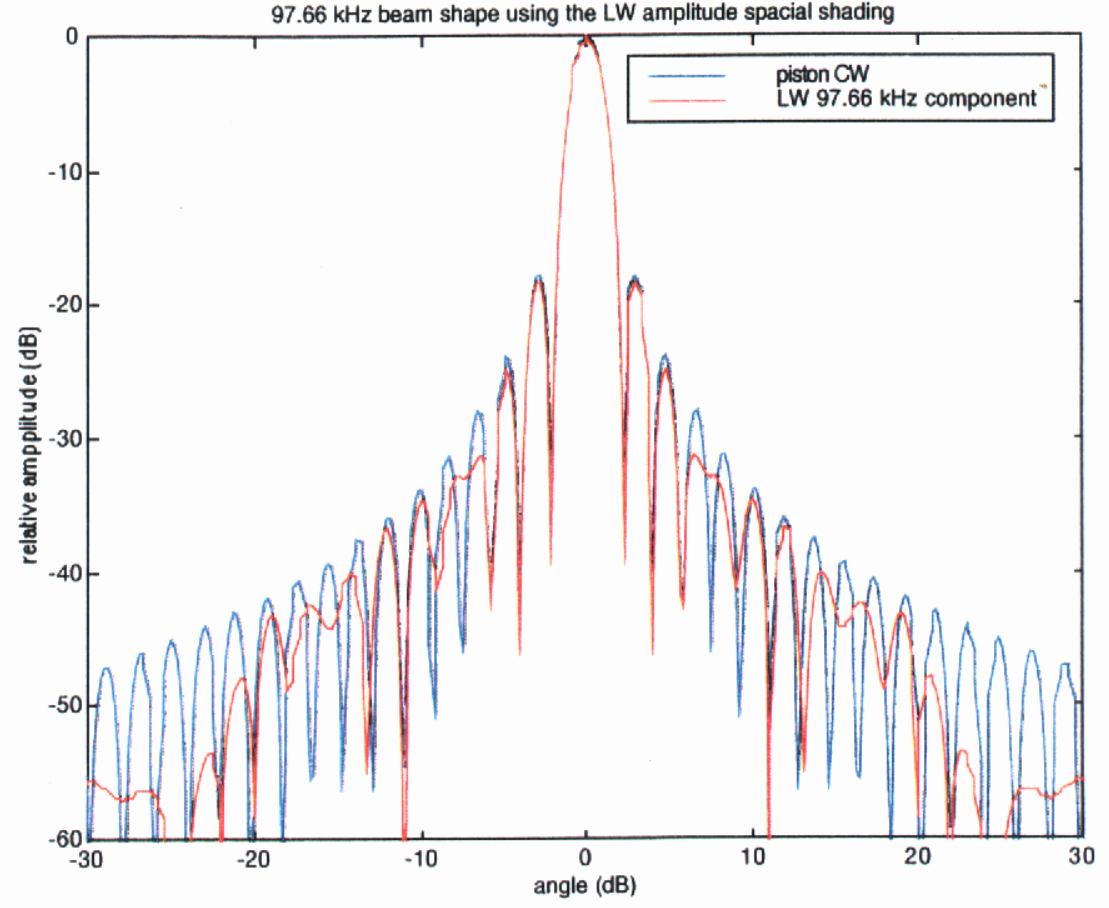

Figure 2. The resultant beam pattern for a $19.53 \mathrm{kHz}$ tone burst from a 0.4 $m$ diameter 24 ring array. The ring contributions were created using the MPS-1 pulse designed for this array, and the single frequency components were spatially filtered but summed with a zero relative phase.

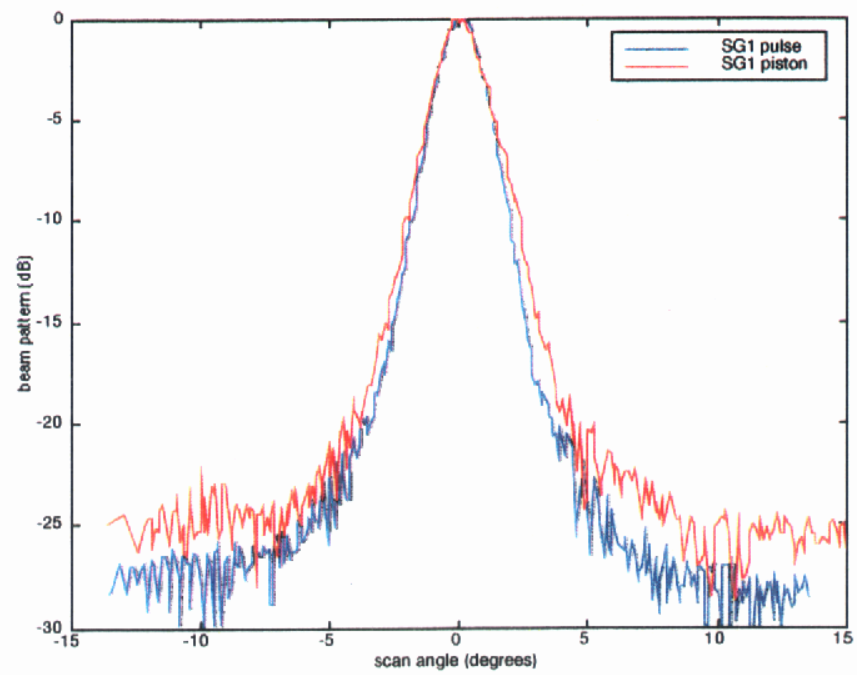

Figure 3. The beam shape comparison for a piston and a Localized Wave pulse. This is experimental data. Both beams contain the same frequency bandwidth in the beam center, but the LW pulse is more contained, with a tighter half width at half maximum and a lower overall level off axis.

There is no efficiency information in this simple example, it is a beam shape comparison. But it is apparent that if an equivalent amount of energy is launched in each 
case, this energy must be going to the center lobe more efficiently in the LW case than in the piston case.

It is the summation of these slight increases in efficiencies that allow the segmented ring array to out perform a piston with a broad band width pulse

\section{Experiment}

\section{IV.1 Equipment and System}

The system was composed of 3 parts, (1) a signal output system which launched 24 separate signals to the array, (2) a signal input system which digitized the input voltages, and (3) a position controller which moved the array into measurement position and controlled the depth, tilt and axial angle. The signals to be launched were put into section (1) and the data to be analyzed taken from section (2).

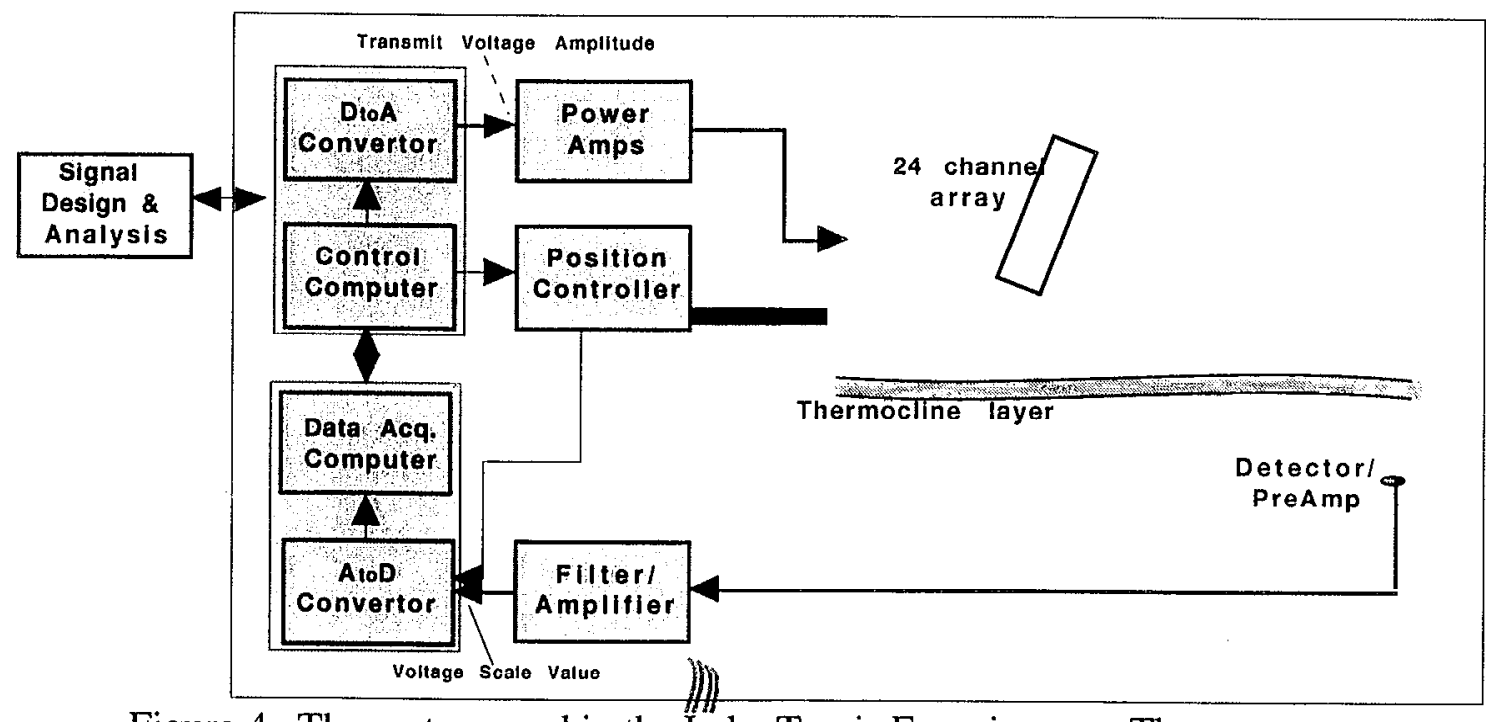

Figure 4. The system used in the Lake Travis Experiments. The source voltages were measured at the input of the Power Amps and converted to head drive voltages using the measured impedances of the array rings and a simulation of the transmission line from the Power Amps to the array. The drive currents were measured at the array head and corrected for their travel through long cables by the same transmission line simulation. A more detailed drawing of the system is contained in Appendix B.

Section (1) was composed of 12 dual output D to A converters and 2 multiplexer units capable of 24 inputs each all driven by proprietary software. The output signals were read into the system memory and output to a set of 24 power amplifiers.

These signals, typically in the 10 s of volts, were then sent down the feed cables to the array. A 100:1 sample of the signal voltage was taken before the power amps and through a multiplexer circuit sent to the data acquisition computer's A to D converter. Additionally, a 20:1 sample of the current to each of the array rings was measured in the head and that voltage sent over cables to another multiplexer for measurement by the Data Acquisition computer. The array driven by this electronic system was a sheet of 1-3 composite piezoelectric driver segmented into 24 rings of equal radial width.

Section (2) was another PC with a LabView program controlling a dual channel A to $\mathrm{D}$ converter. Through changing a switch, the input could be changed from the source voltage and current to the signal from the far field detector. The gain of the A to D converters was controllable through a variable in the panel settings, and was automatically recorded with each data set. 
The receiver was a PVDF patch and preamp which was calibrated for amplitude to a series of standard Navy transducers. A complete engineering drawing of the system detail is available in Appendix $B$.

\section{IV.2 System Characterization:}

Our system was designed to be extremely wide band width, with a low voltage output, 10 s of volts, to reduce distortion. Two tests were conducted to determine the system response.

Since some of the tests are a comparison of the system to the predictions of the simulator, a test was performed to see if the simulator was performing correctly. Radiation of Gaussian pulses with different sigmas from a piston was simulated, and the normalized plot of energy on a curve at infinity was compared to the analytic result. Agreement was better than $1 \%$. This confirmed the frequency response and the angular response of the simulator.

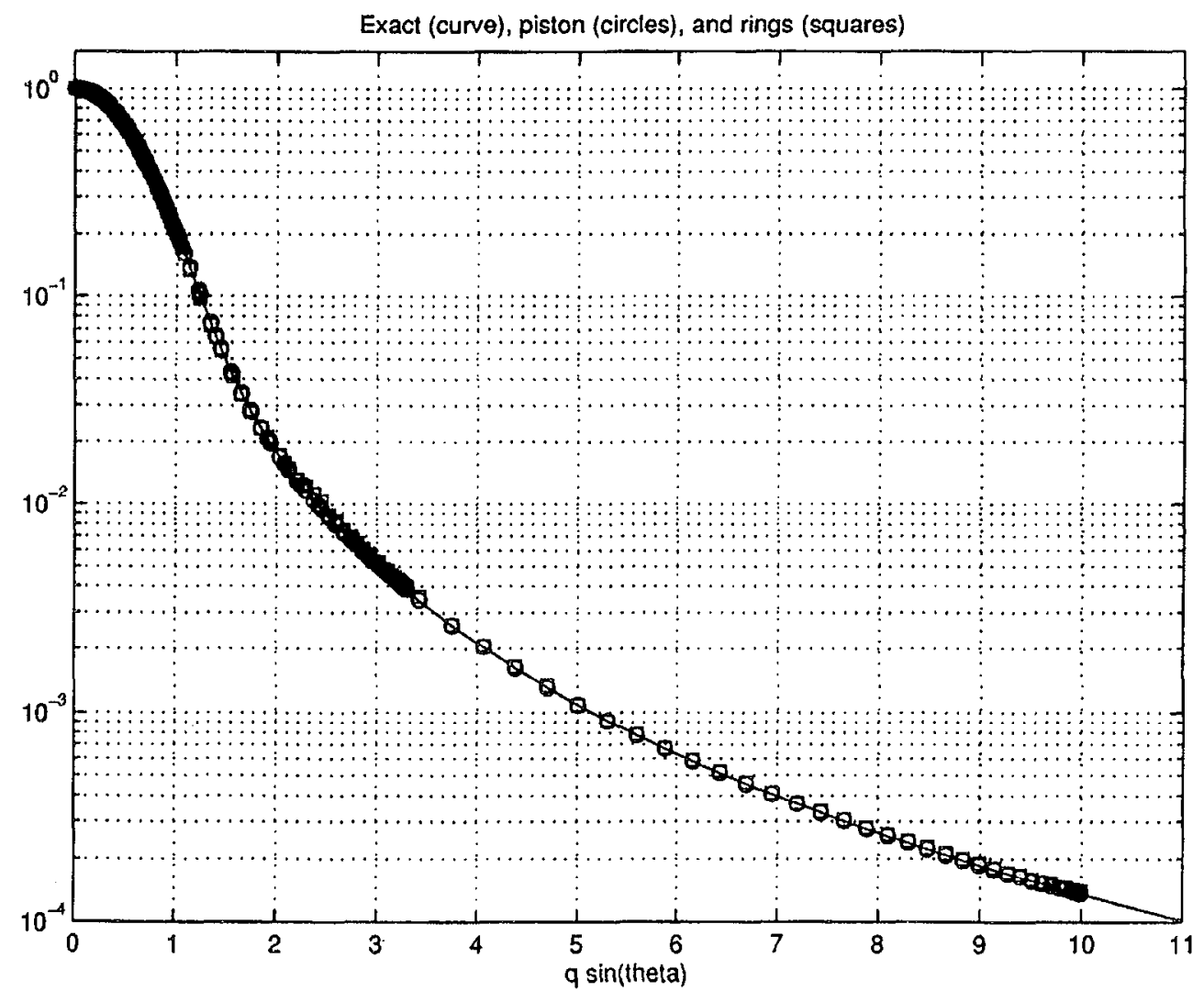

Figure 5. The calibration simulation for the broad band width simulator used in these experiments. This nondimensional comparison shows the field response expected and that predicted for a piston and segmented rings driven as a piston.

Our first test was comparing the beam pattern expected from the $0.2505 \mathrm{~m}$ radius array for several single frequency tone bursts of 10 cycles duration. The frequencies chosen were $20 \mathrm{kHz}, 100 \mathrm{kHz}$ and $240 \mathrm{kHz}$, since these were respectively near the lower end, the middle and the upper limit of the pass band.

The beam patterns were compared to the predicted results of the simulator as well as the expected intensity patterns from $\mathrm{J} 1(\mathrm{z}) / \mathrm{z}$. This was a check on both the performance of the array and the simulator. 


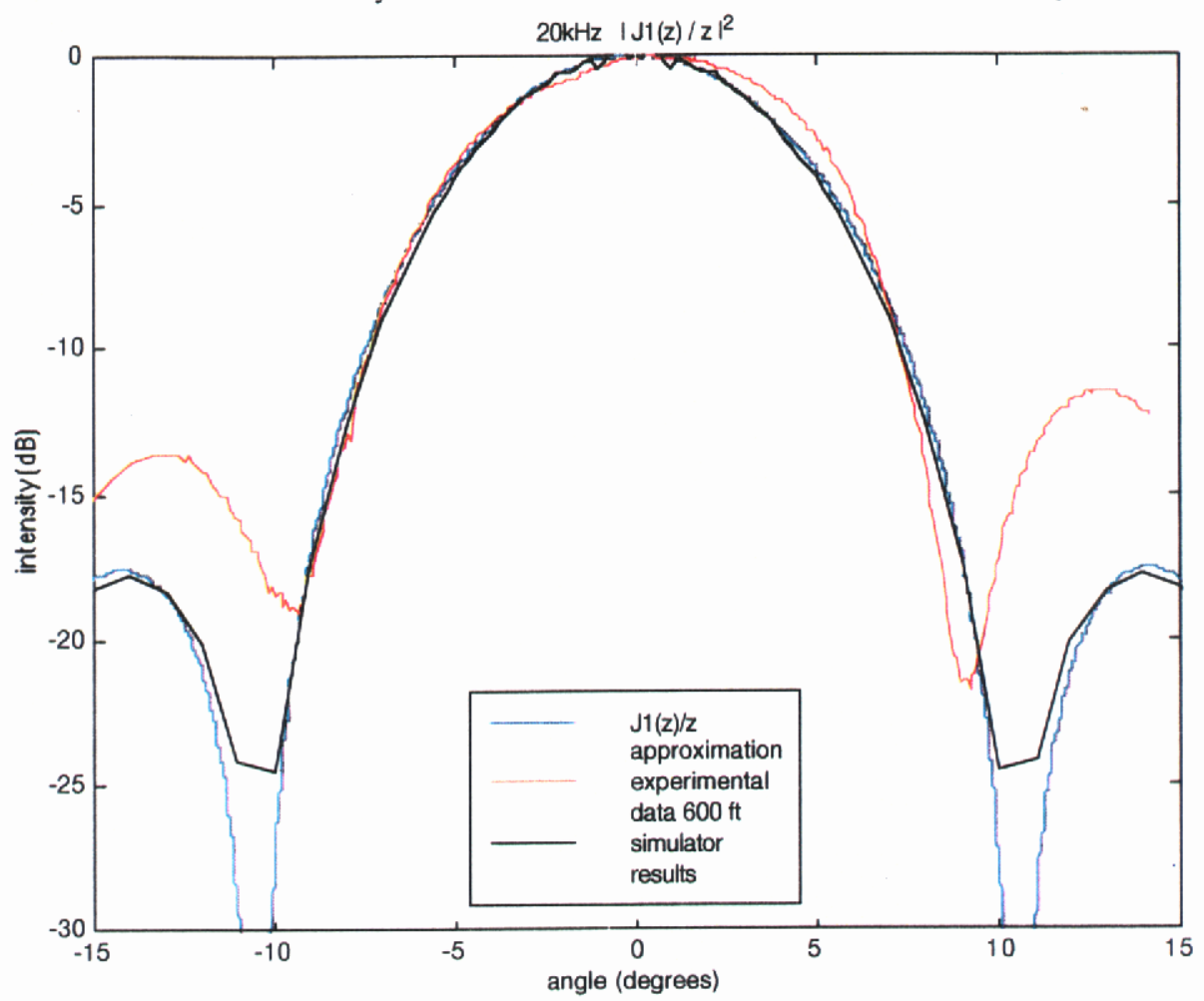

Figure 6a

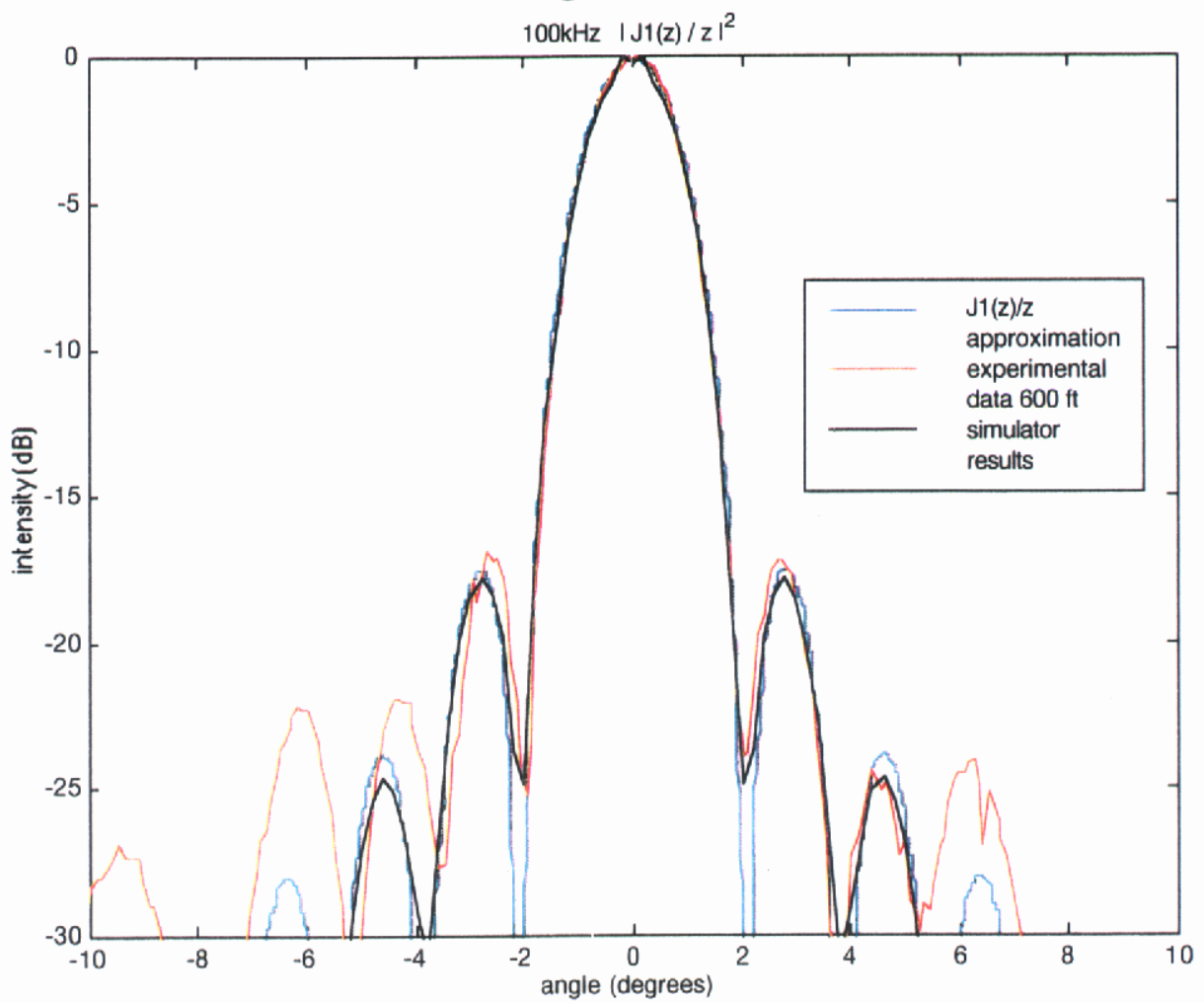

Figure $6 \mathrm{~b}$ 


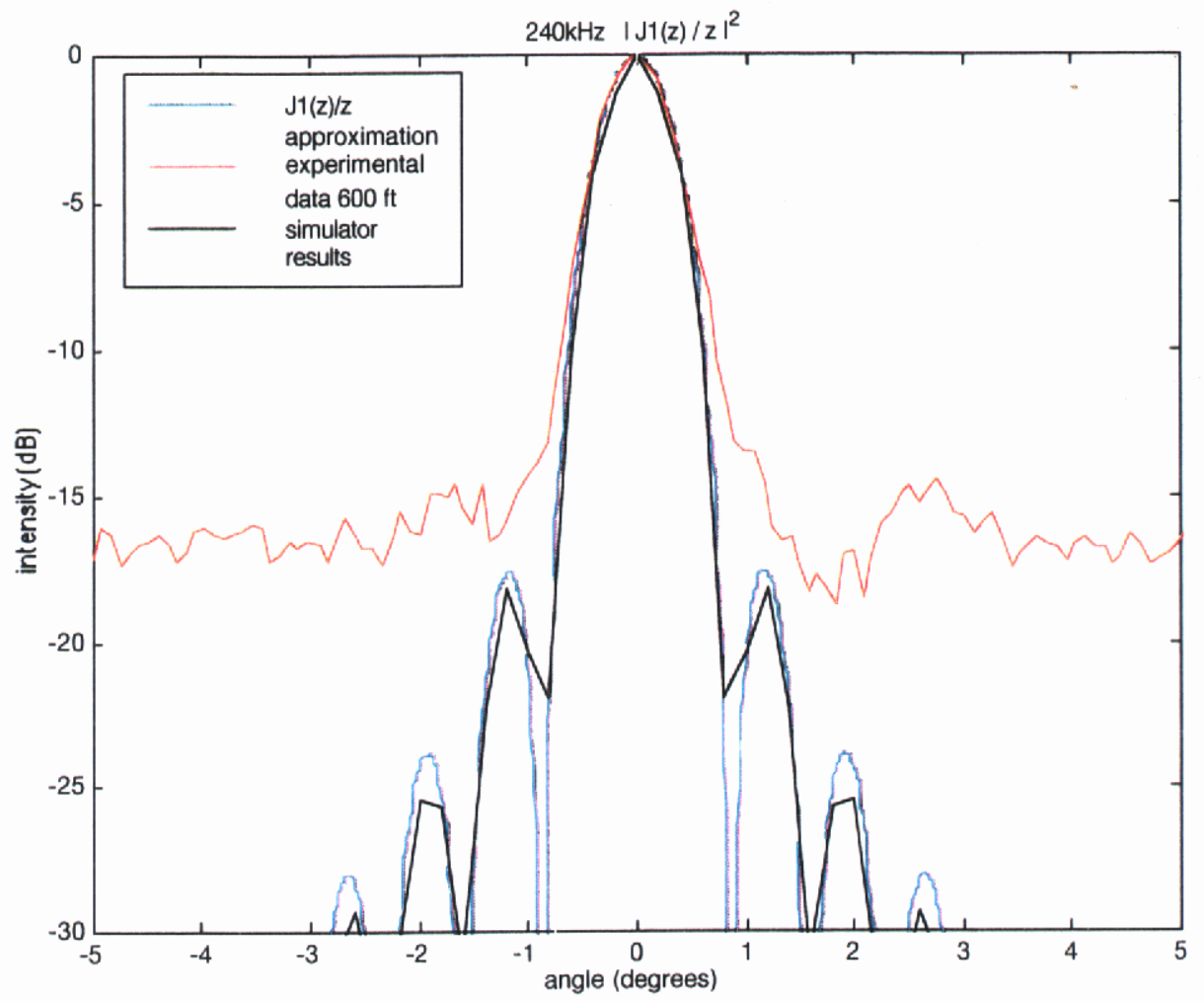

Figure 6c

Figure 6. The comparison of the measured beam pattern to the simulated beam pattern as well as the expected beam pattern using $\mathrm{J}_{1}(\mathrm{z}) / \mathrm{z}$. The 3 beam patterns shown are from -15 to 15 degrees for the $20 \mathrm{kHz}$ beam, -10 to 10 degrees for the $100 \mathrm{kHz}$ beam, and -5 to 5 degrees for the $240 \mathrm{kHz}$ beam respectively for clarity. The vertical scale is 0 to $-30 \mathrm{~dB}$ in all cases. The sampling of the simulator, shown in black, is more coarse than the expected value, but matches the expected pattern remarkably well.

The second characterization was the system response measurement. We use the comparisons of the measured fields at 600 feet to the predicted fields generated by the simulator. The 3 cases used for this test are the MPS1 pulse, which has an effective frequency of $22.9 \mathrm{kHz}$, the SG1 pulse, which has an effective frequency of $46.8 \mathrm{kHz}$, and the MPS3 pulse, which has an effective frequency of $231 . \mathrm{kHz}$.

As seen in the figure below, all 3 cover the frequency range from 10 to $300 \mathrm{kHz}$ with detectable energy. The 3 circles mark the 3 calibration frequencies we used, at 20 , 100 , and $240 \mathrm{kHz}$. Their spectral response is also shown.

A further voltage only test of the system response is a comparison of the fields measured compared to those expected from an idealized system, one with no bandwidth limits. We expect again to see a characteristic voltage response of the system, along with the low pass filter which is inherent in our passed system. In this test we compare the measured LW pulse to the expected pulse on axis.

We observe. in Figure 8, that all three pulses give the same response within a few $\mathrm{dB}$. There is no single frequency information in this figure, since that has been fully addressed in Figure 7. 


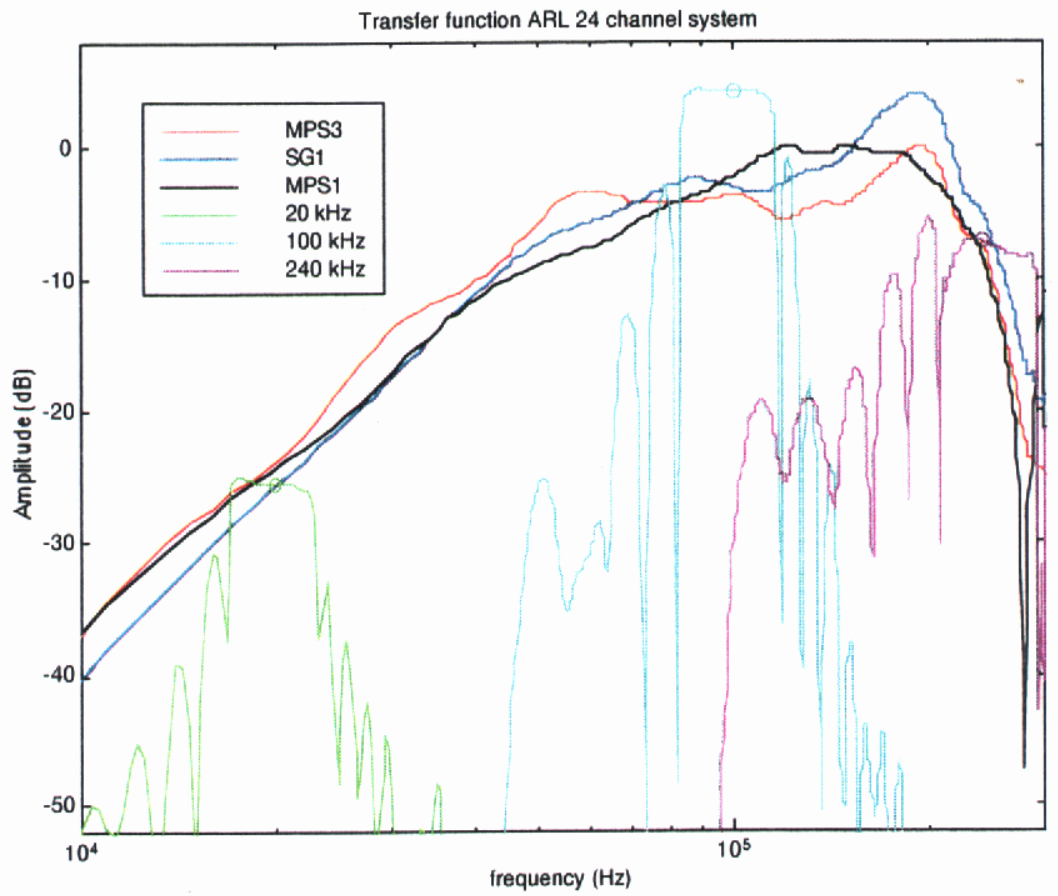

Figure 7. The system transfer function. The vertical axis is in arbitrary units as a check for spectral shape, and the $\mathrm{x}$ axis is 10 to $300 \mathrm{kHz}$. The spectra of the tone bursts have been calculated and then fit to the other curves maintaining their relative levels to the other tone bursts. The circles on the $\mathrm{CW}$ spectra indicate the measured transfer function at the specified frequencies.

This is the on axis response of the highest power signal from the array driven as a piston compared to the input signal. This represents the output voltage of the system for a fixed input voltage over all frequencies. 


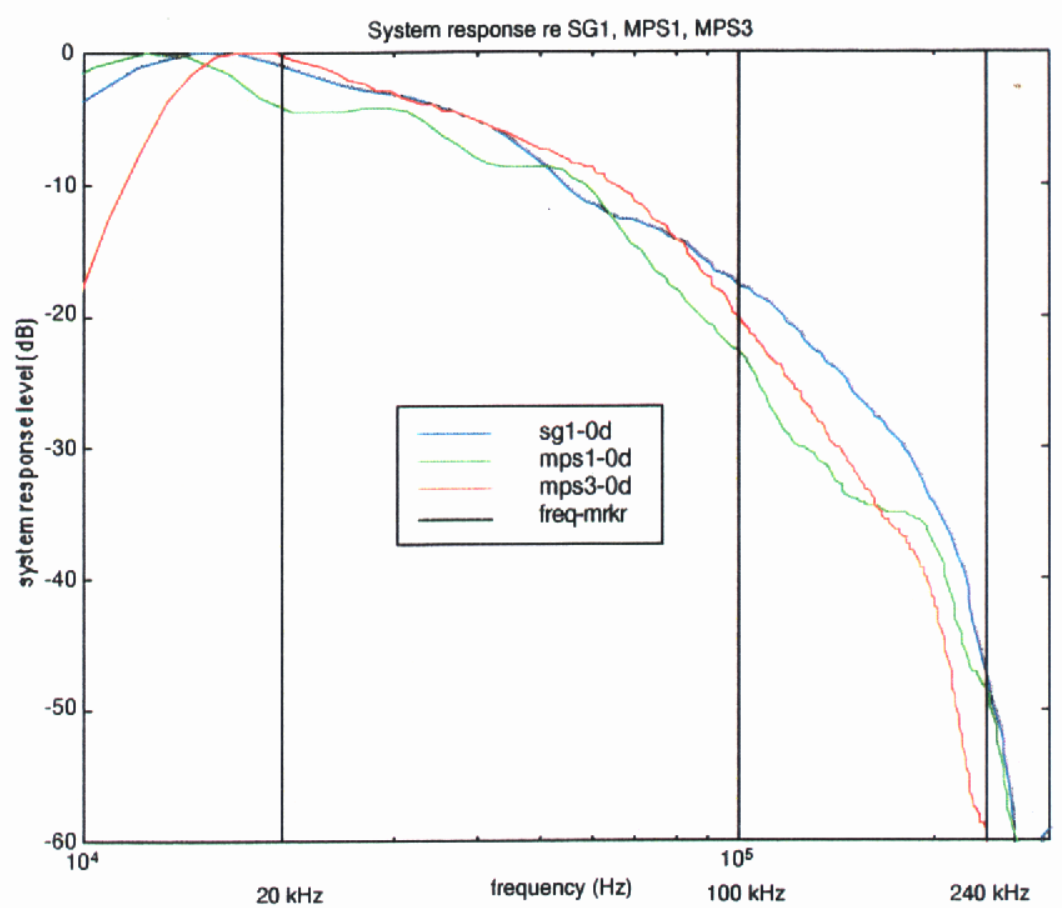

Figure 8. The broad frequency band width response of the system from 10 to $300 \mathrm{kHz}$. This is the measured response compared to the expected response of an ideal transmitter. The response is determined by comparing the experimentally measured on axis signal to the predicted signal for 3 cases; 1) the MPS1 LW pulse, with an effective frequency of $22.9 \mathrm{kHz}, 2$ ) the SG1 LW pulse, with an effective frequency of $46.8 \mathrm{kHz}$, and 3) the MPS3 LW pulse, with an effective frequency of $231 \mathrm{kHz}$. 


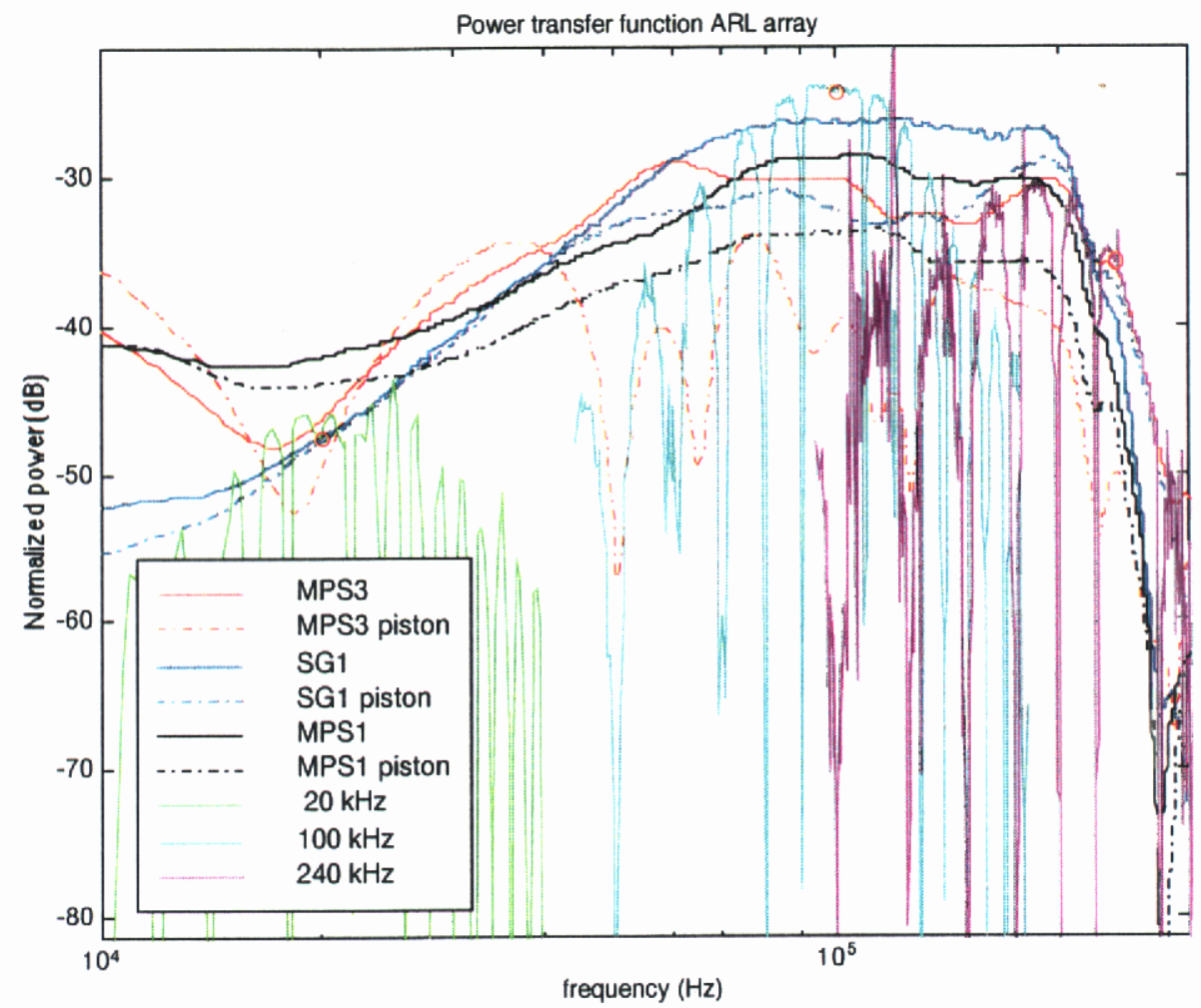

Figure 9. The power transfer function for the system. This is the power spectrum of the measured field signal divided by the product of the voltage spectra and current spectra and summed for all rings, the power received for the power input as a function of frequency. The circles on the $\mathrm{CW}$ spectra show the value at the specified frequencies. Since the previous comparisons of voltage out to voltage in were fairly uniform, this figure suggests that either the current supplied to the array was not accurately produced in all cases or that the environmental effects leading to $+/-6 \mathrm{~dB}$ error bars varied with frequency.

\section{IV.3 Experimental Method}

The source signals were loaded into the source computer and downloaded into the output cards. The cards were held in a PCI bus rack and on queue sent 24 separate signals to 24 power amplifiers, and then down a cable to the array. The output voltage time waveform was measured at the output of the amplifiers, and the currents were measured at the array head by a current loop measurement device. The system is shown in Appendix B.

The signals launched by the array propagated through Lake Travis for either 100 feet to the receiver mounted on the edge of the barge, or 600 feet to the receiver mounted on a tower mounted into the bottom of the lake. As each measurement was made records of the system settings were recorded, either automatically or by notation in the lab book.

The received signals were digitized by the Data Acquisition Computer into time records and then transferred to a third analytical computer for analysis. The signals were also stored on a removable disk for archiving.

The actual measurement of the source voltages and the source currents proved to be a challenge because of the effects of the long cables between the power amplifiers and the projector rings. In the final design, the voltages were measured at amplifier outputs and frequency-dependent corrections were applied (based upon the transmission line equations) to get the ring voltages. The ring currents were measured directly at the projector inputs 
by toroid transformers and current-to-voltage sensing amplifiers, and then transmitted up telemetry cables to the signal recording hardware. Frequency-dependent corrections derived from transmission line equations were applied to correct for these cable effects to compute the ring currents.

As a check on the validity of the ring current and voltage measurements that were made during the lake tests, the ratio of measured voltage to measured current (in the frequency domain, after taking Fourier transforms) was used to calculate the 24 projector ring impedances. The results were compared to the impedance plots of the individual transducer rings that had been measured during fabrication of the array. The results were in good agreement, if allowances were made for the effect of some random noise in the lake test measurements.

The monitoring of ring current did reveal some electrical defects that developed spontaneously in the projector array. On 22 April, 1998, the current in ring \#14 dropped nearly to zero due to a broken wire in the projector housing. This was repaired, and the erroneous data were flagged. On the following day it was discovered that the impedance of ring \#16 had approximately doubled, and it was determined that stress on the transducer face had caused a portion of the transducer ring to become electrically disconnected from the rest of the ring. This information was forwarded to LLNL, and their analysis indicated that the reduction in drive level due to this defect in 1 of 24 rings would not alter the results enough to justify the delay required for surgical repair of the transducer face. The analysis is shown in Appendix C.

The ring current and voltage data were judged to be of sufficient accuracy. Several measurements were repeated to be absolutely certain that the transmitter current and voltage data were measured under the same conditions of drive voltage level as the received acoustic data. Also, tests at different drive levels confirmed that the input energy was proportional to the square of the drive voltage level, as expected for linear operation.

\section{IV.3.1 Pulse Stretching for Noise Reduction}

Some of the pulse waveforms were also transmitted in a stretched form with the intention of increasing the amount of acoustic energy put into the water, to improve the signal-to-noise ratio. This was done by applying a pulse-stretching filter to each electrical input signal before transmission, and then applying a single pulse-compression filter to the signal at the electrical output of the hydrophone receiver. This stretching and compression procedure is frequently used in sonar and radar systems in circumstances where the peak amplitude is limited either by the nonlinearity of the medium or the by the limited drive level of the power amplifier, the latter being the case here.

Figure 10 describes the pulse stretching and compression processes in a more generic form, for an echo-ranging sonar. The box shows a complementary pair of filters. The first one ("S") has an impulse response that looks like a frequency-modulated (FM) wave, of relatively long duration, sweeping upward across the sonar-operating band. The second filter ("C") has in impulse response that looks approximately like a downwardsweeping FM wave of the same duration. Mathematically, its impulse response is defined as the inverse Fourier transform of the reciprocal, over the operating band, of the Fourier transform of the impulse response of the first filter; i.e., it is a deconvolution filter. The filters can both be scaled so that their gains across the pass band are approximately unity; i.e., they preserve the energy of whatever band-limited signal is passed through them. The more important feature of these filters is that when used in combination, they have essentially no effect on any signal that is confined to the sonar band. 


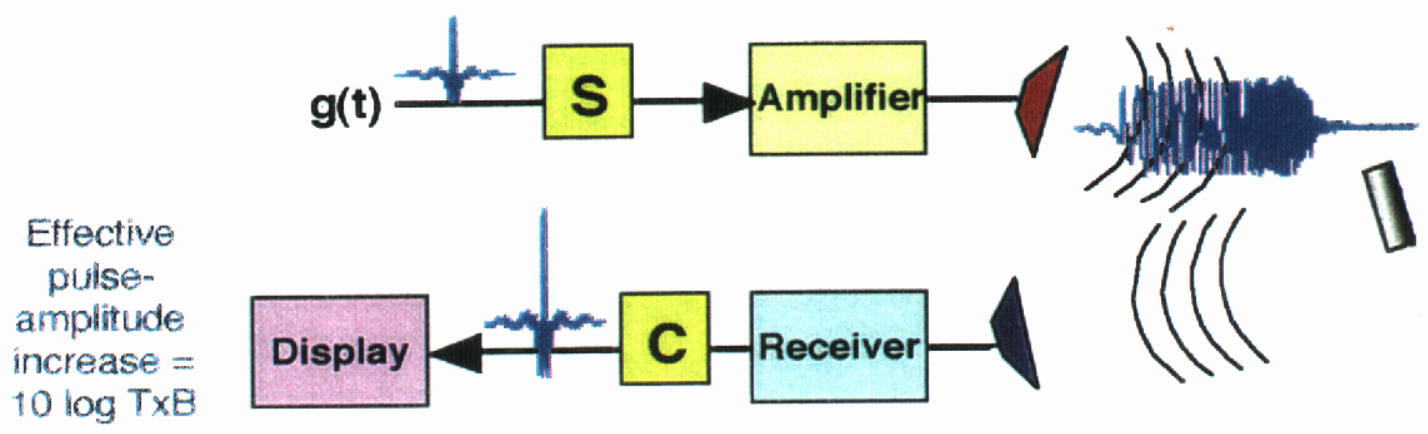

Figure 10. Pulse stretching of a time signal. The original signal, $g(t)$, is convolved with an increasing FM chirp which covers the transmission band width. The resulting signal, with much more energy in it, is then radiated into the water. Upon reception, the field signal is recompressed by convolution with the inverse of the stretching filter, and the result of launching the original signal is obtained.

At this point we make the assumption that the transmission, propagation, echo formation, return propagation, and reception processes all behave as linear, time-invariant systems (at least during the duration of a single transmission event), at least up to the point of the "Display" input as shown. This assumption is well known to apply to linear electronic systems of this simple type, and also to linear elastic acoustic processes in an ordinary environment. Under this assumption the effects of all the system components can be treated mathematically as a sequence of convolution products of impulse responses. Since the convolutions can be done in any order, this means we can move the second filter ("C") farther along in the chain, all the way through the echo process and through the receiver to the Display input.

This situation is depicted in Figure 10. The compression filter " $\mathrm{C}$ " boosts the pulse amplitude to a height larger than what it was. That is the whole purpose of pulse stretching and compression: It has the same effect as using a short pulse of larger peak amplitude that would have violated the limits of either the amplifier or the medium. This makes it easier to overcome noise (whose level is unchanged by the compression filter because it has unity gain across the operating band).

For the Localized Wave experiments described in this report there was no echoproducing object in the acoustic path, and there were 24 signal inputs instead of 1 , but the same principles apply. We assume that, for each of the voltage inputs, the transmission, propagation, and reception behave as a linear, time-invariant system (at least during the duration of a single transmission event), which can be represented as a convolution by an impulse response. Thus, the signal at the electrical output of the hydrophone receiver can be expressed as a summation over all array rings.

However, when pulse stretching was performed, the sequence of events was as follows: Each pulse waveform was convolved with the pulse-stretching filter impulse response and scaled by a gain factor before transmission. Then the signal received at the hydrophone was convolved with the pulse-compression filter impulse response giving a result which is just $\mathrm{K}$ times what was obtained before application of pulse stretching and compression, as desired.

For the localized wave experiments the pulse stretching filter impulse response was defined as follows: First an FM wave of $1 \mathrm{~ms}$ duration, sweeping from $5 \mathrm{kHz}$ to $300 \mathrm{kHz}$, was generated and extended with zeros on each end to make a waveform of $2 \mathrm{~ms}$ duration. 
Then its Fourier transform was computed and normalized to have a constant, unit-gain magnitude over the band $5 \mathrm{kHz}$ to $300 \mathrm{kHz}$, the inverse transform of this frequencydomain function was taken to give. The compression filter impulse response was then defined as the inverse transform within the pass band and 0 outside.

In actual practice the 24 transmitted waveforms were pulse-stretch-filtered beforehand and stored in the computer for later transmission. Comparison tests between stretched/compressed and unaltered waveforms were performed at the $100 \mathrm{ft}$ range, with excellent results. The beam patterns matched in the center where the signal-to-noise ratio was high for both, and the noise floor in the side lobes was seen to be better for the stretched/compressed pulses. However, especially at the $600 \mathrm{ft}$ range, the scale factor $\mathrm{K}$ was inadvertently set too low due to an overly conservative policy for avoiding output "clipping". This caused the stretched/compressed pulses to have an increased energy of less than $2 \mathrm{~dB}$ at the $600 \mathrm{ft}$ range, making them of little or no advantage. Thus, the stretched/compressed pulses proved to work in principal, and to be of potential benefit where peak amplitudes must be limited, but were not demonstrated to be markedly superior at long range, where they were most needed, due to the overly conservative setting of $\mathrm{K}$.

\section{IV.3.2 Environmental Effects}

The accuracy of the test results was influenced by time varying environmental conditions, especially during the final phase of measurements conducted in late April, due to the regular cycle of seasonal springtime warming at Lake Travis. During this season variations in the temperature profiles can produce vertical focusing and defocusing effects that change the received levels by several $\mathrm{dB}$ over the course of a minute. The effect becomes much more pronounced when the acoustic transmission path is long, due to the cumulative effects of refraction.

Fortunately the environmental variations could have no effect upon the measured electrical input energy. The measurement of projector input energy was a very time consuming, one-ring-at-a-time, sequential process. Therefore, the test plan was revised to perform these measurements at separate times rather than to interrupt the acoustic tests. (Such interruptions would have made the acoustic data even more susceptible to the environmental variations discussed in the next paragraphs). Thus, our analysis results use electrical input measurements made on 23 April, and, to a lesser extent, measurements made on 1 April and 2 April.

Significant environmentally-induced variations in received acoustic level were seen in the Localized Wave transmission tests, especially during late April for the $600 \mathrm{ft}$ transmission path. Fluctuations in the apparent elevation angle were also observed when performing the axial alignment procedure. This alignment procedure made use of corner elements that had been incorporated into the projector design for this purpose. A special setup of the waveform generation computer caused two identical pulses to be transmitted, first from one of the upper corner elements and then from the lower corner element underneath it, at a time $\tau$ seconds later. The two received pulses were then displayed with a time offset of $\tau$ on a CRT so that they overlaid perfectly if the transmission delays were identical. If not, the elevation angle of the projector array housing was adjusted until they did match cycle for cycle, to within an accuracy of \pm 0.5 microseconds. By this method, the face of the projector was aligned to be orthogonal to the sound path with an elevation accuracy of about $\pm 0.1^{\circ}$, assuming a vertical separation of 17 in between the corner elements. Horizontal axial alignment was done in similar fashion but was not as critical, since acoustic data were collected continually as the projector housing was swept through azimuth.

The variability of the vertical refraction conditions was revealed by the need for frequent readjustments of vertical aiming angle as indicated by the axial alignment procedure. In a typical data-taking run, the received acoustical energy was measured as the projector was swept slowly in azimuth to produce a beam pattern, so that its peak would 
give the measured voltage from the receiver. Azimuth sweeps were done at two speeds, to give "coarse" and "fine" beam pattern plots. To insure that the projector's elevation angle was set correctly the axial alignment procedure was performed shortly before the azimuth sweeps, and repeated fairly soon afterward. For most of the azimuth sweeps the projector was initially pointed at the receiver, then swung to one side and swept slowly from left to right. In some cases the data recording began before the projector was swung to the left, so that the recording sometimes included two separate times when the projector was pointed at the receiver, and any discrepancies in recorded acoustical energy could be measured. These discrepancies, which were presumably due to environmental variations, were sometimes less than $0.5 \mathrm{~dB}$, but were often 2 to $4 \mathrm{~dB}$, and occasionally $8 \mathrm{~dB}$ or more. Since, for mechanical reasons, the time interval between these acoustic energy level measurements was often considerably less than the time interval between the axial alignment procedure and the on-axis acoustic energy measurements, it is possible that the azimuth sweeps sometimes "missed" the on-axis energy peak (due to vertical refraction) with a discrepancy greater than these values. It is hard to quantify the errors precisely, but based upon the observations our subjective estimate is that measurements of acoustical energy had environmentally-induced error magnitudes of 0 to $8 \mathrm{~dB}$, with random variations between those limits, but with the probability distributions weighted more heavily toward the lower end (i.e., smaller errors).

Based upon these numbers, errors of up to $16 \mathrm{~dB}$ are possible when comparing measured efficiency ratios for any two waveform conditions. However, much smaller errors are more likely. We estimate that the errors in such a comparison have an uncertainty of $\pm 6 \mathrm{~dB}$.

\section{IV.3.3 Pulse Comparisom Experiments}

Part of the purpose of the experiments was to compare the result of the Localized Wave Pulses to equivalent piston launched broad band width beams. The question to be answered was whether the expense of a multi-channel system including an individually addressable array would be justified by improved performance.

To make this comparison, some choice of a single pulse signal must be made. For our purposes, the choice of pulse was left to ARL:UT, and they choose the center signal of several Localized Wave pulses. The reason for this choice is that the center function is essentially an envelope of all the other pulse spectra, without the characteristic "missing frequencies" of the other rings of the Localized Wave. This is shown in the source spectra shown in Appendix F.

\section{Data analysis}

\section{V.1 Amplitude Correction}

Three corrections were made to the digitized data, two of them previous to analysis, and one afterwards. The first two corrections are multiplication by the 'volt scale A or B', the amplitude parameter stored automatically when the data is taken, and multiplication by the transmit voltage, a parameter that must be read from the Output computer and entered into the Data Acquisition computer. The first parameter is the attenuation used to make sure that the data fills most of the binary levels in the A to D converter. The second is essentially the volume control for the output voltages.

The third correction is only performed on the measurements of the array head voltages and currents. It compensates for the changes made to the voltages (current measurements are presented ad voltages as well) by traveling through the known lengths of Belden cable.

\section{V.2 System Compensation}

Any system acts on an input signal by convolving its own impulse response into the input signal. For our 3 stage system, shown below, $y=h 3 \cdot h 2 \cdot h 1 \cdot x$, where ${ }^{\prime} \cdot$ denotes 
convolution. In out case, we model the response of each stage as a time derivative and an accompanying impulse response $\eta$.

The response of all three stages is in general a fairly simple function of amplitude and phase as functions of frequency. If we can determine these components, then we can separate out the function $\mathrm{h} 1 \cdot \mathrm{x}$ to achieve the optimum field in the water, and correct measurements for h2 and h3 effects on those fields.

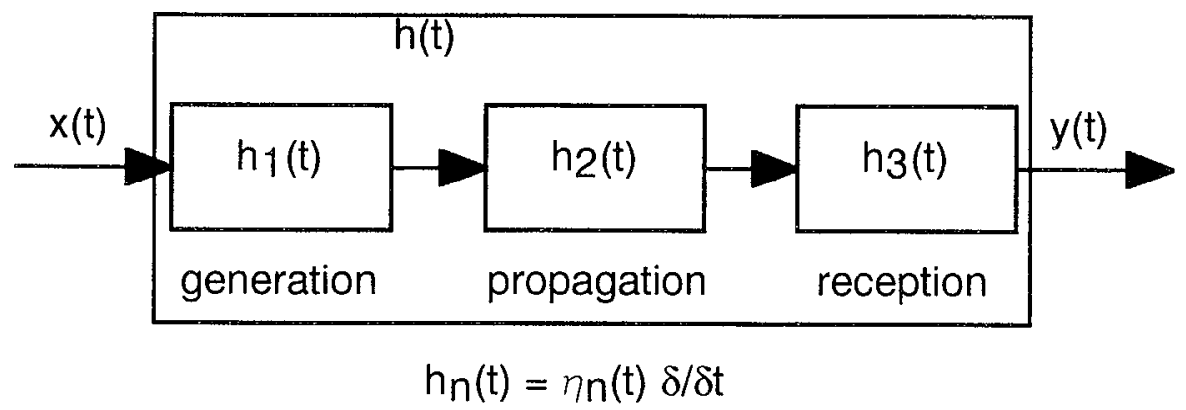

Figure 11. The system model for signal pre and post processing. Each stage is modeled as a frequency dependent amplitude and phase change and a time derivative, following well established theory. The goal is a complete determination of $\mathrm{hl}(\mathrm{t})$ in order to launch the necessary fields to create our pulse.

In practice this demands that the receiver be completely characterized over its entire bandwidth for both amplitude and phase. In our work this was impossible, so we did several different compensations, one which made the signals appear to be a second derivative of the input signal at 100 feet, one that yielded a third derivative, and one which made no corrections at all to the signals.

The results of these compensations are denoted as MPS-10d, 2d, 3, SG1-0d, 2d, $3 \mathrm{~d}$, and so on. The resulting fields had different beam patterns and efficiencies, as shown in the data. 


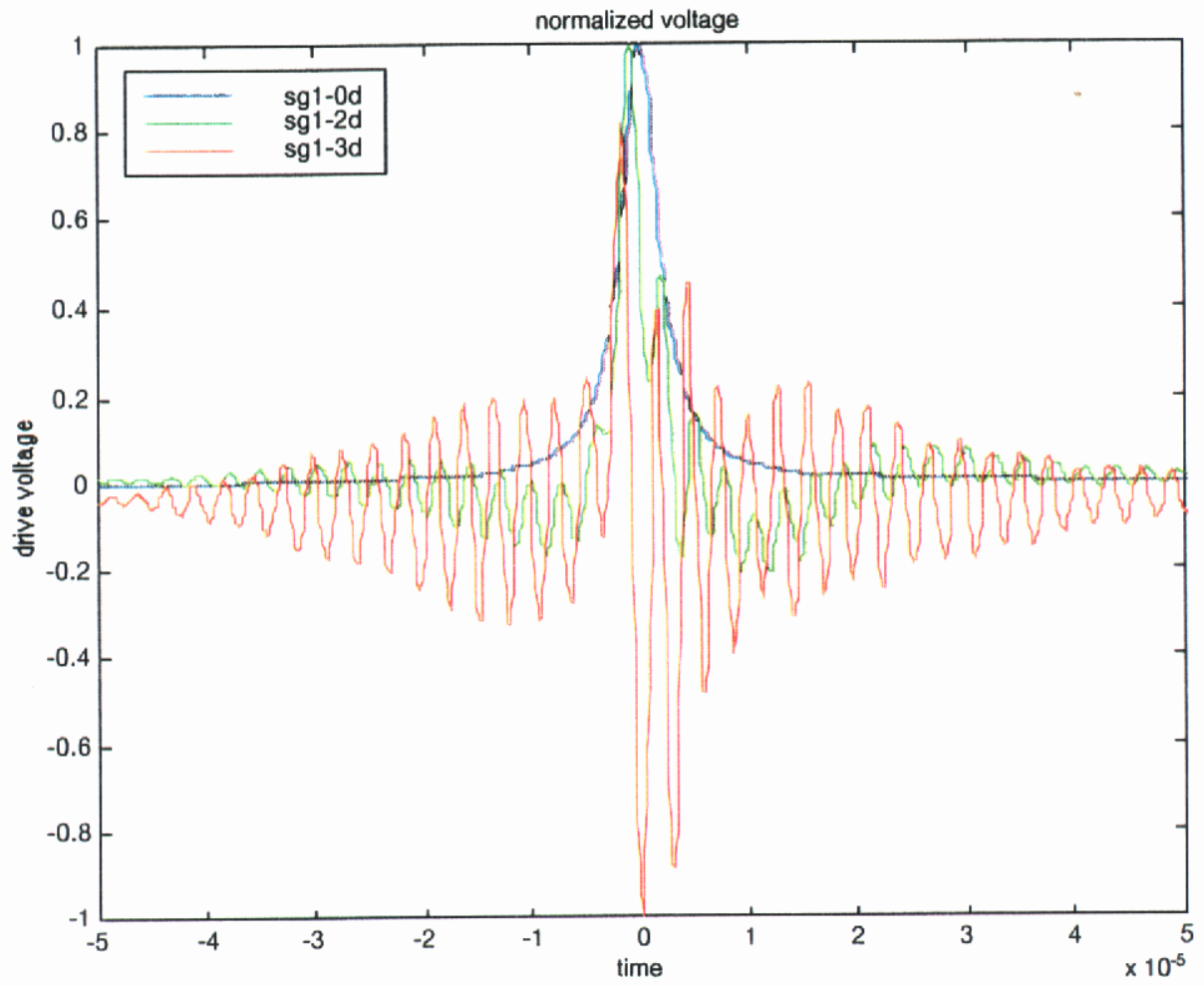

Figure 12. The signal for SG-1 with no compensation, second derivative compensation, and third derivative compensation. The total energy in the signals varies over a factor of approximately 2 for a fixed input level. Each signal would result in a different field being created in the water at the face of the array.

\section{V.3 Time Window Resizing}

Time windowing is used in the data analysis to reduce the signal to noise ratio.

Since all of the signals had a noise base, analysis of the entire record would yield both the desired signal and the noise. The noise would increase with window size.

The array was mounted on a pivoting tower with a standoff holding the assembly so that the angular rotation of the pivot was not centered on the face of the array. This means that the first and last possible signals, an impulse from the nearest and farthest points, varies as the array is rotated. It also introduces a slight inaccuracy in the measurement angle. The mounting and path lengths are shown in Figure 13. 


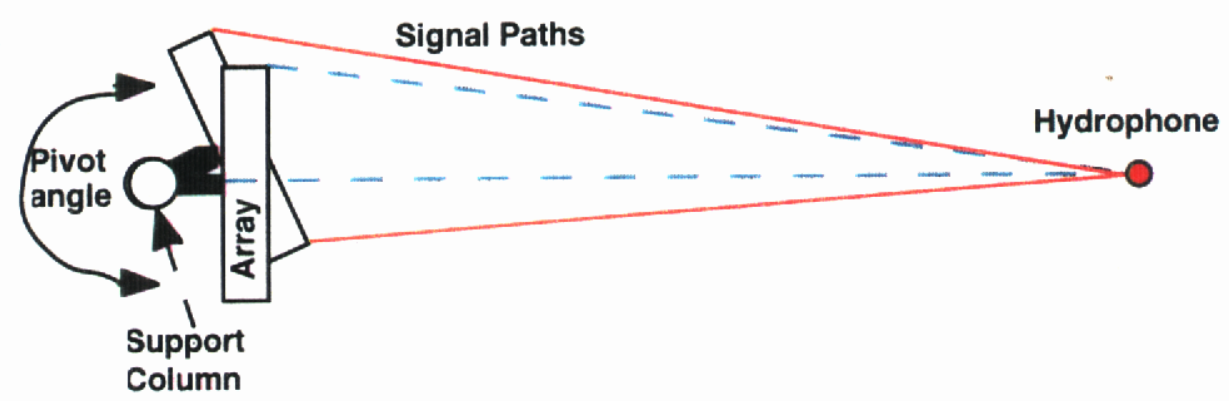

Figure 13. The mounting diagram shows the path length for 2 different angle orientations. Coupled with the actual expected width of the signal, the time gate, used to reduce the signal to noise ratio in the measurement, must be changed as the angle changes.

As an illustration, a data set with its variable time window is shown in Figure 14. The beginning of the time record is set to zero for clarity, and the axes are angular measurement number, horizontally, and relative time, vertically. Horizontally, the signals are those of an alignment, fast sweep to -90 degrees, slow scan through 0 to 90 degrees. The window width is shown as well as the background noise in the lake.

The figure shows the absolute value of the signals detected. The maximum signal strength occurs during the alignment, around signal 1 , and around measurement 250 , as the array sweeps through 0 degrees again.

Figure 15 shows the layout of the data. It is the integral of the square of the time gated signal, in this case a stretched signal and it's recompressed signal, as a function of measurement number. The lower part of Figure 15 is the angle corresponding to the measurement number.

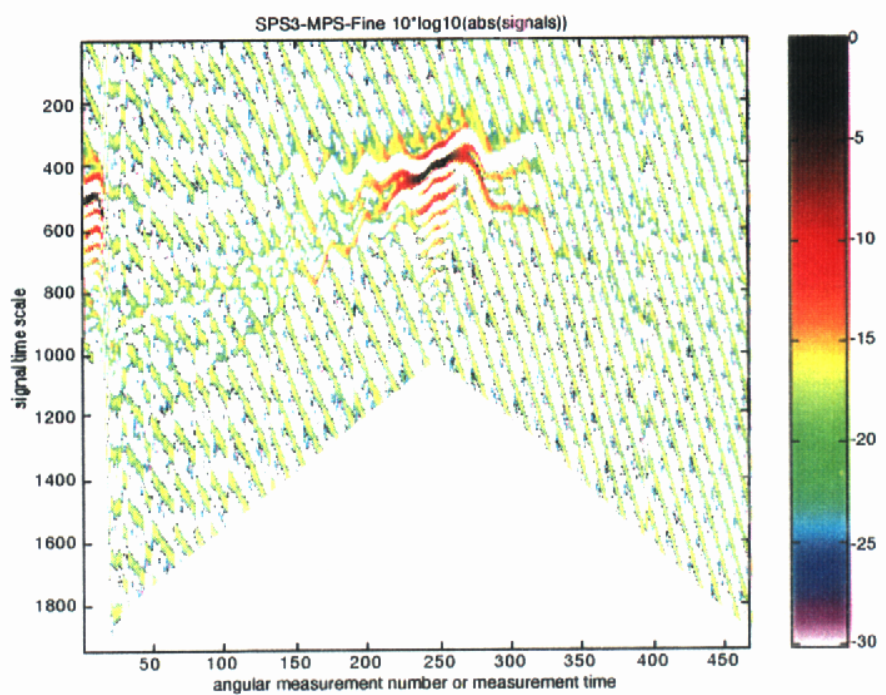

Figure 14. The time gate varies with angle to compensate for the geometry of the array and to assure that no significant part of the signal is missed. The axes are angle measurement number, horizontally, and relative time, vertically. 

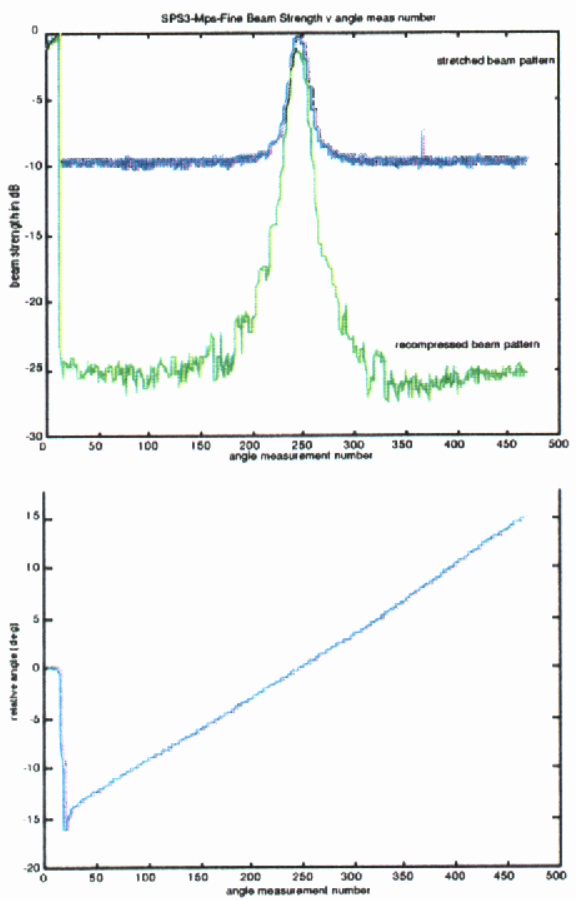

Figure 15. A data layout for a typical experimental run, in this case a stretched pulse. The data in the top picture is the stretched pulse energy as a function of measurement number in blue, and the recompressed pulse, with a higher signal to noise ratio, in green. The figure at the bottom is the angle corresponding to the measurements above. The angles start out at the left for the alignment measurements, swing off axis very quickly, and then swing back through the alignment position and off to the other side.

\section{Results}

\section{VI.1 Comparison to tone bursts}

The following data is a graphical representation of the relative efficiencies of the LW pulses, identified in the horizontal figure axis caption, compared to a single tone burst, which is identified in the vertical axis caption. Each tone burst is a single frequency sine wave which is ten cycles in length. Thus the tone burst signals are of different lengths for the different frequencies, but contain the same number of oscillations.

The figures are representations in $\mathrm{dB}$ units, and are over a $20 \mathrm{~dB}$ range in all cases for consistency. It should be remembered that on the basis of previously described analysis, we have error bars on these results of $+/-6 \mathrm{~dB}$ in all cases.

\section{VI.1.1 $100 \mathrm{kHz}$ tone burst observation}

During the experiment it was observed that the relative efficiency of the $100 \mathrm{kHz}$ tone burst was unexpectedly high. This led to an analysis, detailed in Appendix A, which showed that if a tone burst at or near the maximum of the power transfer curve is chosen, a higher efficiency than that of any broad bandwidth pulse can be achieved.

Strictly speaking, this tone burst must have an amplitude and phase distribution which matches that of the broad band pulse. It should not be surprising that a piston tone burst, which has uniform amplitude and phase, would also achieve a similarly high efficiency.

Efficiency is not the only measure of importance in most applications though. The narrow bandwidth and side lobe levels of a tone burst would not support some applications, such as precision ranging, or high band width communication. 


\section{VI.1.2 100 ft. Data Results}

The LW pulses compared in this section were the Modified Pulse Spectrum pulse 1 (MPS1), and the Superposed Gaussian pulse 1 (SG1) with compensation or pre-correction as described above to make the received signal look like the 2 nd derivative of the input signal at a receiver position 100 feet away from the array (mps1-2d and sg1-2d), the $3 \mathrm{rd}$ derivative of the input signal (mps1-3d and $\mathrm{sg} 1-3 \mathrm{~d}$ ), and the results of adding no compensation whatsoever (mps1-0d and sg1-0d).

Compensating the pulses in this fashion was somewhat arbitrary since at 100 feet we were still in the near field of some of the pulse frequency components, and since we could not separate out the effects of the receiver from the radiator and medium. Still we can see that the $3 \mathrm{~d}$ compensation, which tries to launch a beam containing more high frequency energy than either the $0 \mathrm{~d}$ or $2 \mathrm{~d}$ case, yields lowered efficiency as we would expect based on the prior comparison of the expected to actual fields measured.

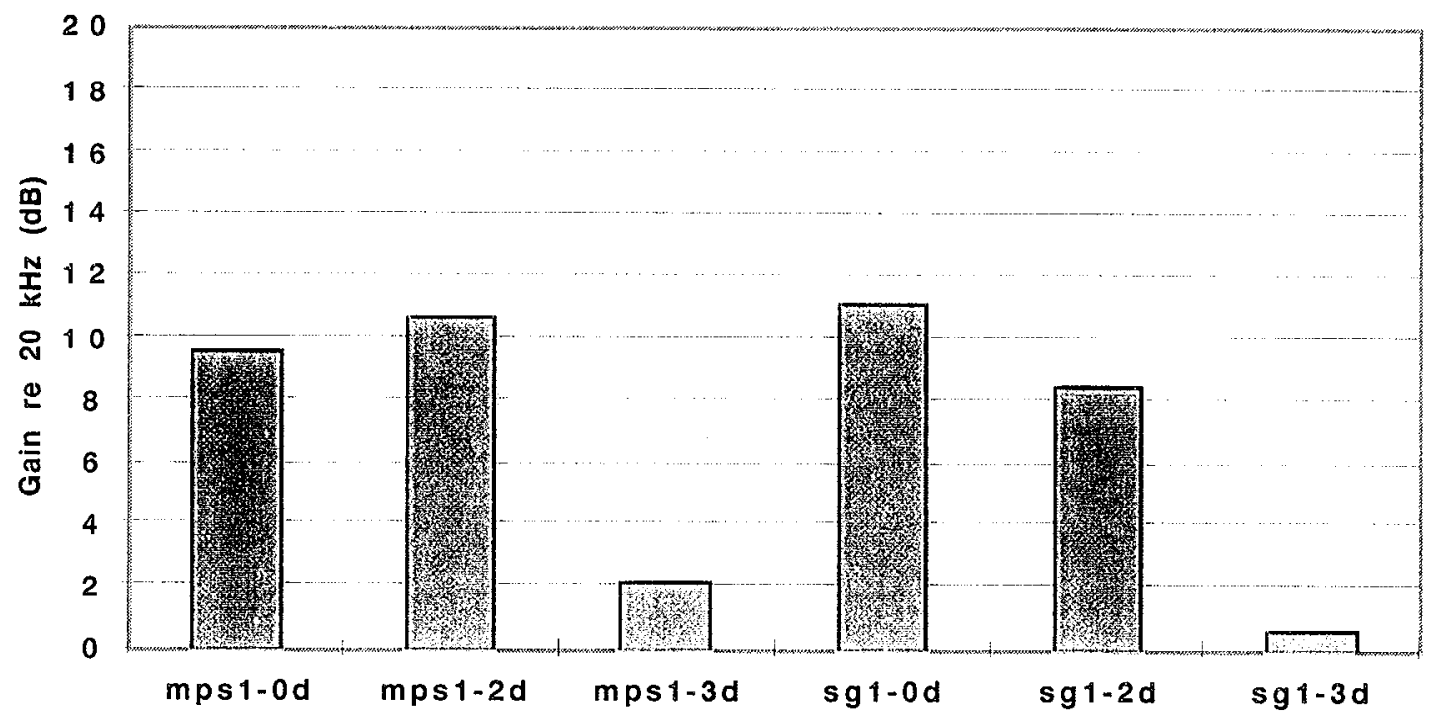

Figure 16. Relative gain of the different $\mathrm{LW}$ pulses relative to the $20 \mathrm{kHz}$ tone burst at 100 feet. The vertical axis is drawn for $20 \mathrm{~dB}$ for consistency. 


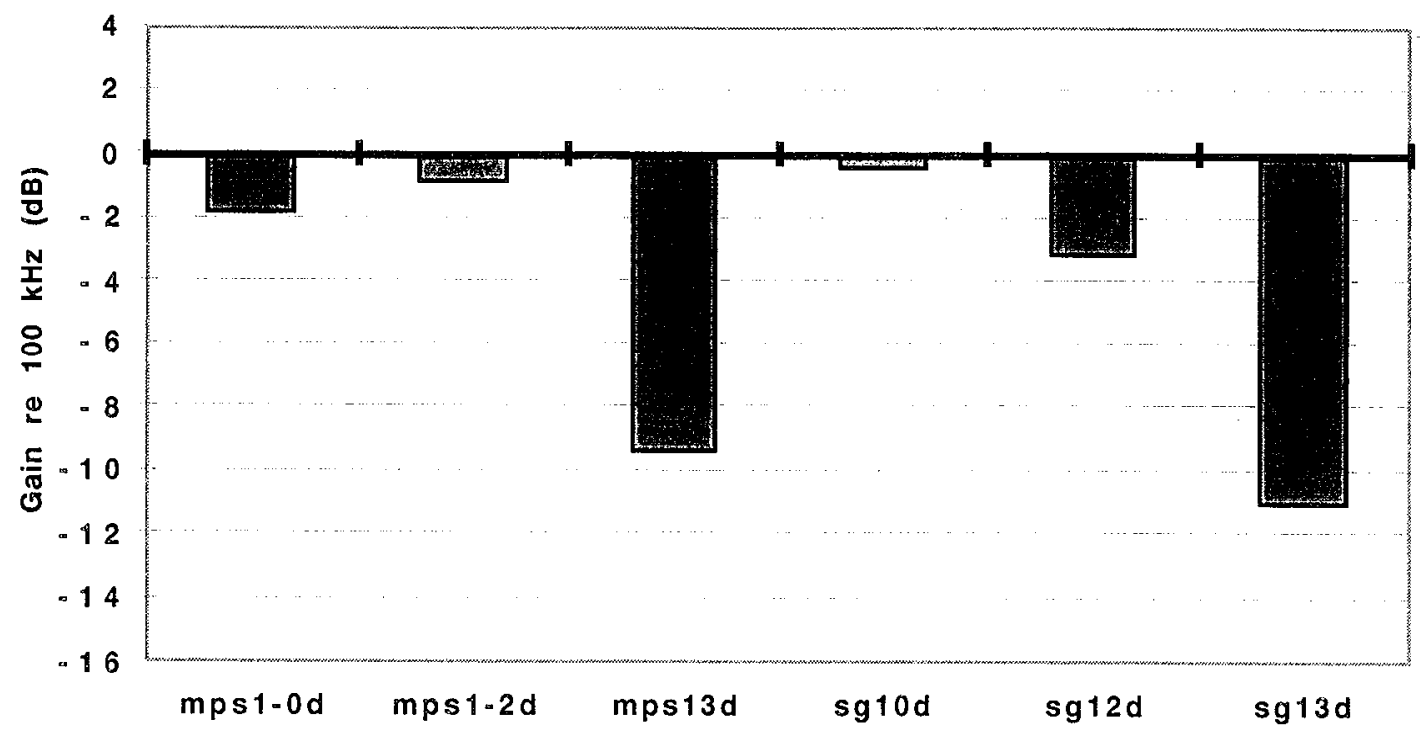

Figure 17. Relative loss of the different $\mathrm{LW}$ pulses relative to the $100 \mathrm{kHz}$ tone burst at 100 feet. The vertical axis is drawn for $20 \mathrm{~dB}$ for consistency. The data for $100 \mathrm{kHz}$ fits like a key in a lock with either the 20 or $240 \mathrm{kHz}$. The increased efficiency of the $100 \mathrm{kHz}$ tone burst is addressed in the text. and Appendix A.

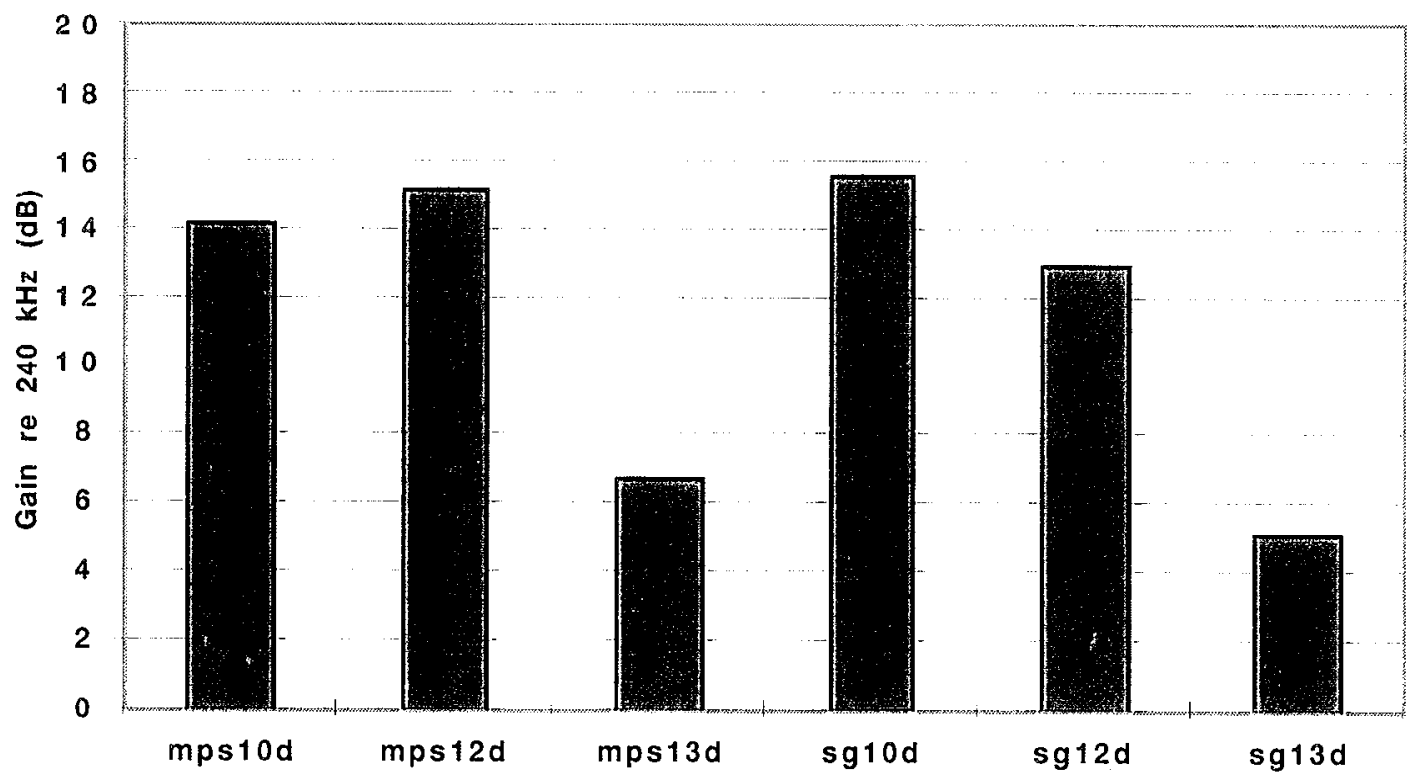

Figure 18. Relative gain of the different LW pulses relative to the $240 \mathrm{kHz}$ tone burst at 100 feet. The vertical axis is drawn for $20 \mathrm{~dB}$ for consistency.

\section{VII.1.3 $600 \mathrm{ft}$. Data}

For the true far field measurements at $600 \mathrm{ft}$., a reduced set of waveforms were chosen as a result of time constraints. These waveforms were mps $1-2 \mathrm{~d}$ and sg1-0d, both of which were launched at $100 \mathrm{ft}$. as well, and a new waveform, mps 3 based on a different set of design parameters for the Modified Pulse Spectrum. The effective frequency of 


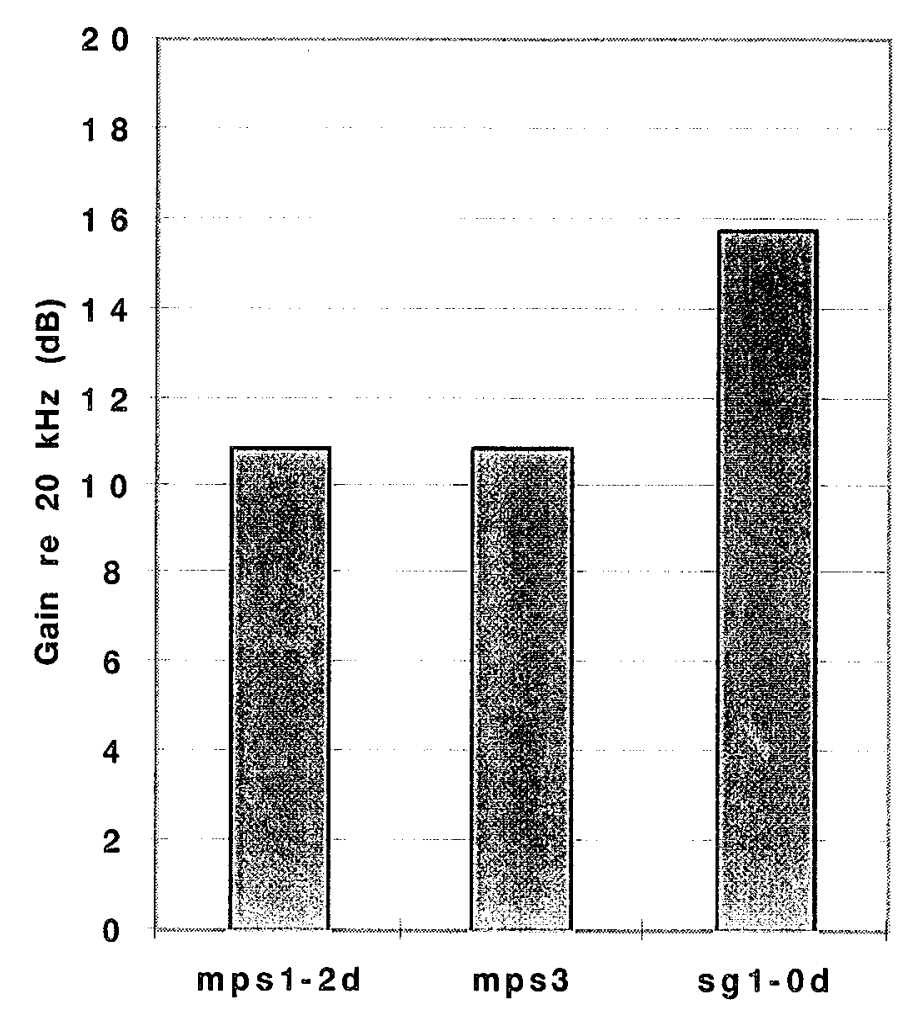

Figure 19. Relative gain of the different $\mathrm{LW}$ pulses relative to the $20 \mathrm{kHz}$ tone burst at 600 feet. The vertical axis is drawn for $20 \mathrm{~dB}$ for consistency. The 3 beams are the best beams measured at 100 feet, and a new beam designed for optimal beam width, the MPS3 with a very high effective frequency of $231 \mathrm{kHz}$.

mps 3 was $231 \mathrm{kHz}$, where the effective frequency is defined as the first moment of the combined spectrum sent to the array. It is interesting to note that while it performed as well as mps 1-2d relative to the tone bursts, it was surpassed by the uncompensated Superposed Gaussian sg 1-0d which had a much lower effective frequency. MPS3 also performed less well relative to its equivalent piston than either $\operatorname{sg} 1-0 \mathrm{~d}$ or mps1-2d, to be shown in the next section, probably because of its much higher equivalent frequency source functions. 


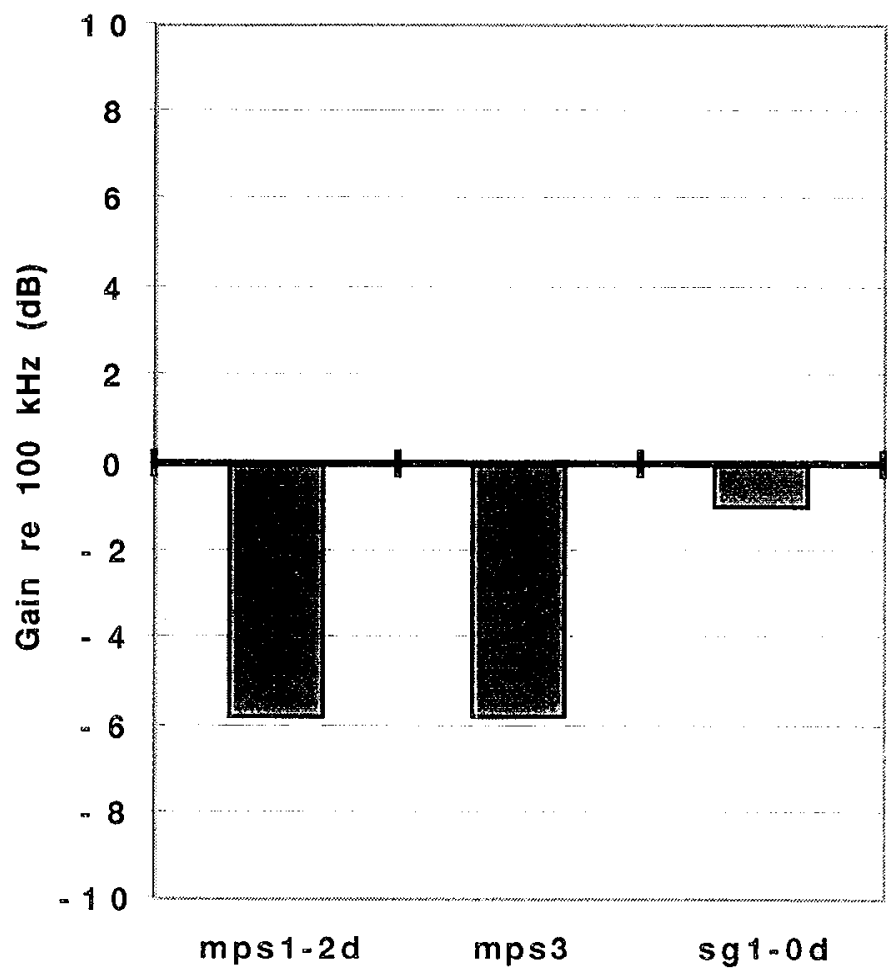

Figure 20. Relative loss of the different LW pulses relative to the $100 \mathrm{kHz}$ tone burst at 600 feet. The vertical axis is drawn for $20 \mathrm{~dB}$ for consistency. The increased efficiency of the $100 \mathrm{kHz}$ tone burst is addressed in the text and Appendix A. 


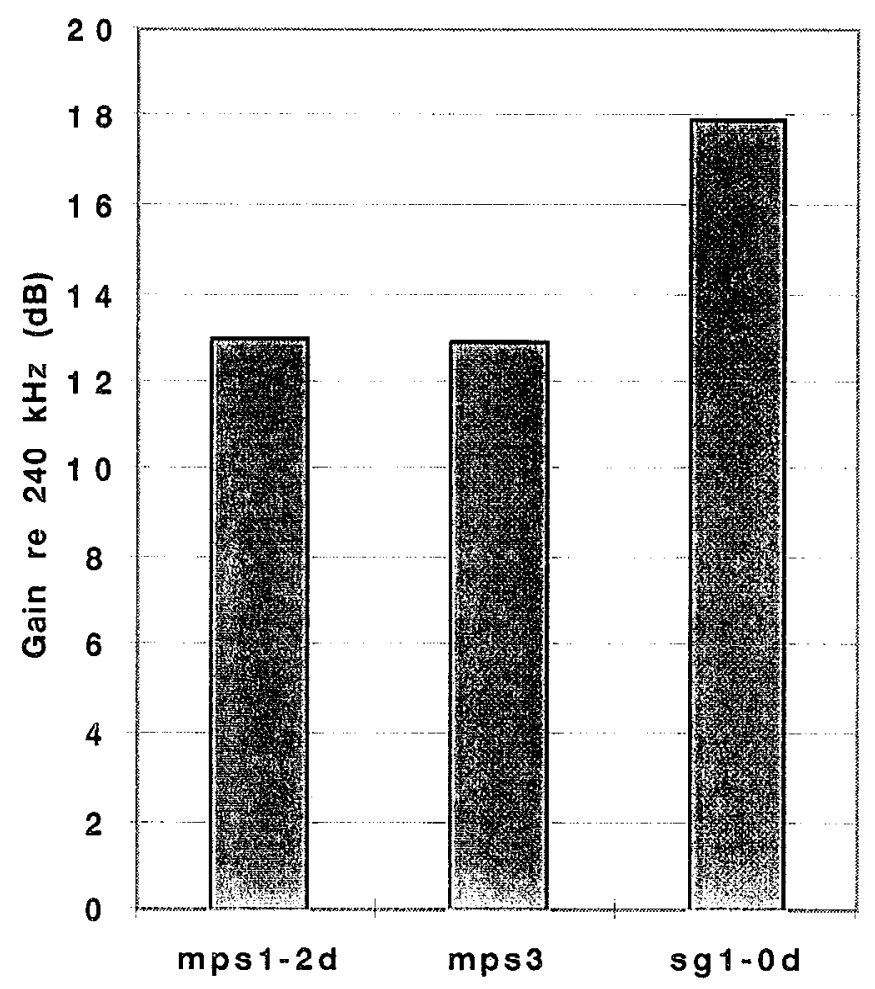

Figure 21. Relative gain of the different LW pulses relative to the $240 \mathrm{kHz}$ tone burst at 600 feet. The vertical axis is drawn for $20 \mathrm{~dB}$ for consistency.

\section{VH.1.4 Comparisons to Pulses.}

Relative efficiencies were compared of LW pulses to their equivalent piston pulses. The equivalent piston pulse was defined as the result of driving the entire array with the source function fed to the center element when the LW pulse is created. This seemed a logical choice since in general the center function contains all the frequencies of the $\mathrm{LW}$ pulse, and none of the missing frequencies that the outer rings exhibit.

Again time constraints dictated that only a few of the pulses launched could be repeated as piston pulses, and the only LW pulse which can be compared at both 100 and 600 feet is mps $1-2 \mathrm{~d}$. Remembering the $+/-6 \mathrm{~dB}$ error bars we have imposed on the data, there is very little we can say conclusively.

It is interesting to note that the fact that sg 1-0d performed better than mps $1-2 \mathrm{~d}$ against the tone bursts did not imply that it would perform better against its equivalent piston pulse. It is also interesting to note that as mps1-2d progressed from 100 to $600 \mathrm{ft}$. its relative efficiency apparently increased, expected if mps1-2d extended the near field as expected, since the piston would not be expected to extend the near field. But again, the error bars we impose on the results preclude any conclusions. 


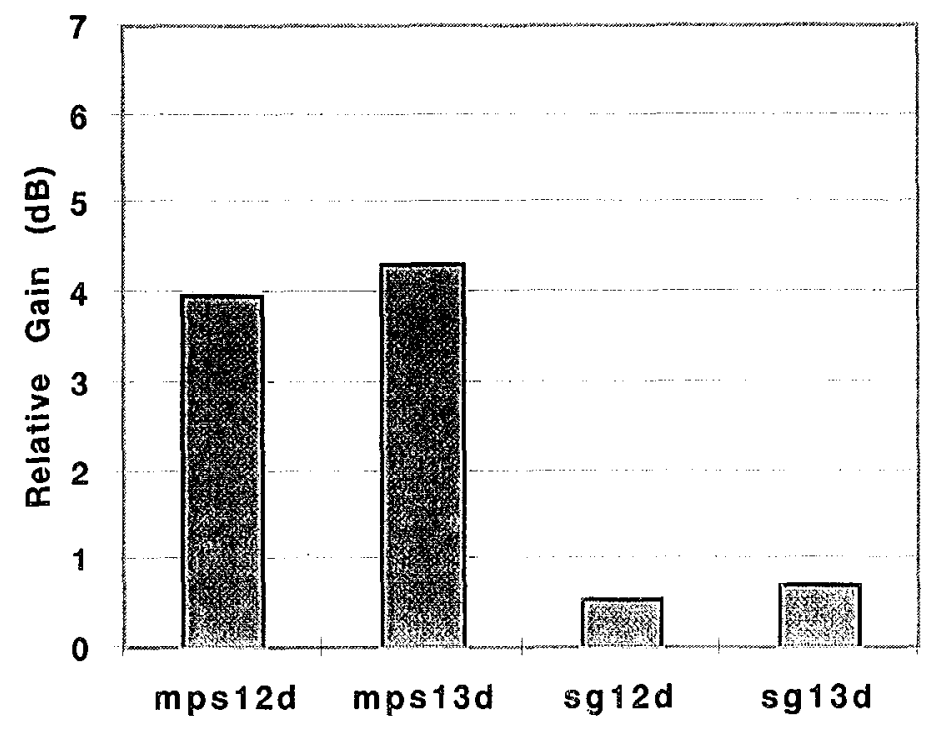

Figure 22. Comparison of Localized Waves to equivalent piston pulses at the 100 foot range. The vertical scale is in $\mathrm{dB}$ of gain over the equivalent piston pulse. The 4 beams tested at this range were MPS1 with a compensation to make the measured signal be a second derivative and a third derivative, and the SG1 pulse with the same compensation.

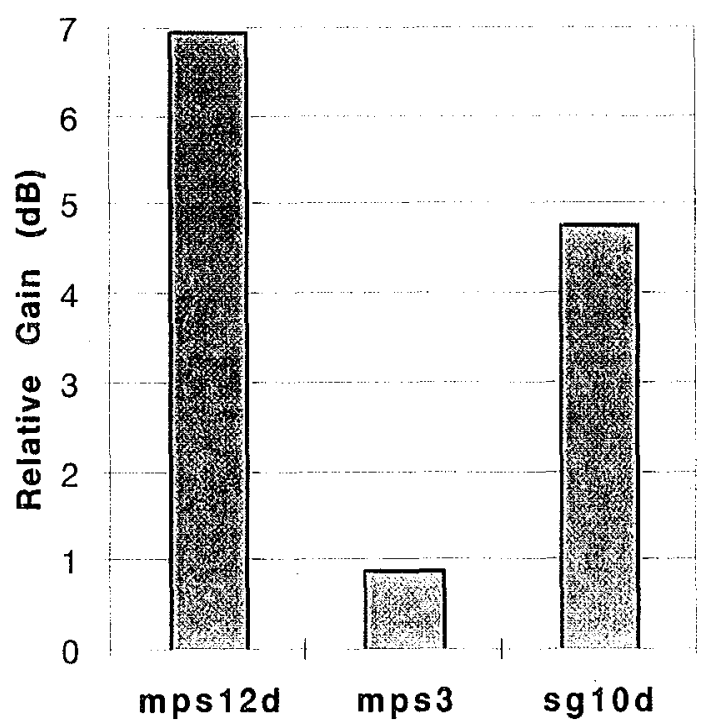

Figure 23. Comparison of Localized Waves to piston pulses at the 600 foot range. The vertical scale is in $\mathrm{dB}$ of gain over the equivalent piston pulse. The 3 beams tested at this range were MPS1 with a compensation to make the measured signal be a second derivative, the MPS3 pulse with no compensation, and the SGl pulse with no compensation. 


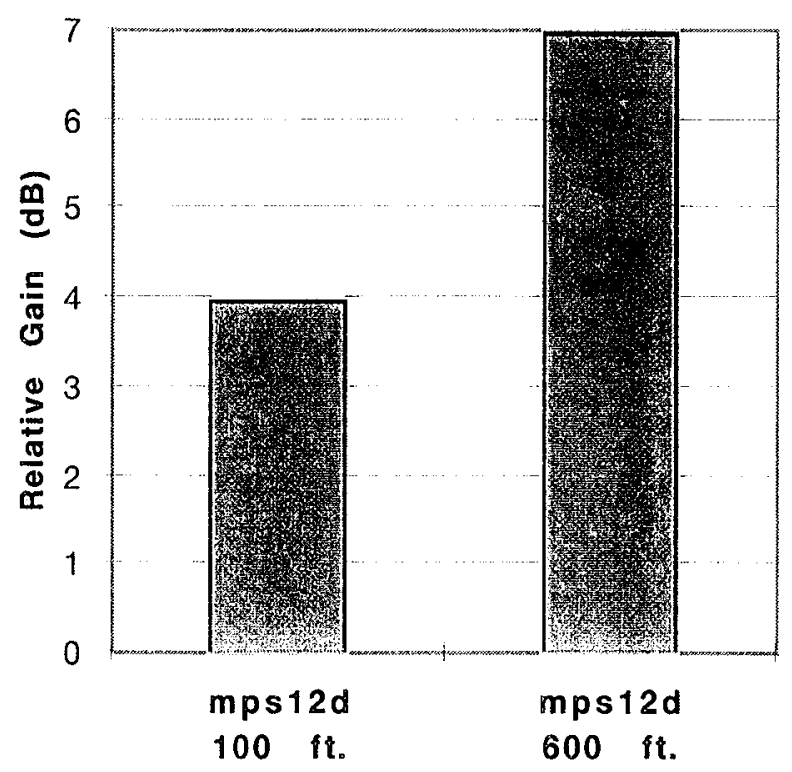

Figure 24. As the MPS1 pulse propagated, it became more efficient relative to the piston, suggesting that at 100 feet the piston beam was still in the near field, but spread naturally becoming less efficient than the LW extended near field pulse as it passed into the 600 foot range.

\section{Tabulated Data}

The graphical energy efficiencies presented above were arrived at by calculating the relative normalized energy gain on axis for all the experimental beams at two down range distances. In addition, the beam widths, in full angular width at the $-3 \mathrm{~dB}$ point for each beam is presented in Table 2 .

\section{VIII.1 Relative Energy}

This section contains the on axis measurements used to calculate the above graphical presentations of the comparison results. To arrive at the graphs, we use a time based procedure. First, the on axis energy was calculated by squaring and integrating the time signal measured at the down range detector. The input energy is arrived at by multiplying the source voltage by the source current for each of the 24 rings power signal, then these are summed and integrated over time. Next the source energy is subtracted from the on axis energy to get the relative energy gain in $\mathrm{dB}$, and the ratio of these numbers are plotted above. 
Lawrence Livermore National Laboratory

University of Texas at Austin

\begin{tabular}{|c|c|c|c|c|c|}
\hline $\begin{array}{l}\text { Signal } \\
\text { name }\end{array}$ & $\begin{array}{l}\text { LLNL } \\
\text { On Axis }\end{array}$ & $\begin{array}{l}\text { ARL } \\
\text { Input }\end{array}$ & $\begin{array}{l}\text { LLNL } \\
\text { On Axis }\end{array}$ & $\begin{array}{l}\text { ARL } \\
\text { Input }\end{array}$ & $\begin{array}{l}\text { Difference } \\
\text { LLNL-ARL }\end{array}$ \\
\hline $\begin{array}{l}\mathrm{tb} 020 \_100 \\
\text { tb020_en } 100\end{array}$ & $\begin{array}{l}-44.92 \\
-30.78\end{array}$ & $\begin{array}{l}-43.5 \\
-30.9\end{array}$ & -14.13 & -12.6 & -1.53 \\
\hline $\begin{array}{l}\mathrm{tb} 020 \_600 \\
\mathrm{tb} 020 \_ \text {en600 }\end{array}$ & $\begin{array}{l}-66.62 \\
-30.95\end{array}$ & -63.0 & -35.67 & -32.1 & -3.57 \\
\hline $\begin{array}{l}\text { tb100_100 } \\
\text { tb100_en100 }\end{array}$ & $\begin{array}{l}-44.32 \\
-41.73 \\
\end{array}$ & $\begin{array}{l}-42.0 \\
-42.0 \\
\end{array}$ & -2.59 & 0.0 & -2.59 \\
\hline $\begin{array}{l}\text { tb100_600 } \\
\text { tb100_en600 }\end{array}$ & $\begin{array}{l}-60.96 \\
-42.01\end{array}$ & $\begin{array}{l}-58.0 \\
-42.0\end{array}$ & -18.95 & -16.0 & -2.95 \\
\hline $\begin{array}{l}\mathrm{tb} 240 \_100 \\
\mathrm{tb} 240 \_\mathrm{en} 100\end{array}$ & $\begin{array}{l}-63.28 \\
-44.66\end{array}$ & $\begin{array}{l}-60.0 \\
-44.6\end{array}$ & -18.63 & -15.4 & -3.23 \\
\hline $\begin{array}{l}\mathrm{tb} 240 \_600 \\
\mathrm{tb} 240 \_ \text {en600 }\end{array}$ & $\begin{array}{l}-82.31 \\
-44.53\end{array}$ & $\begin{array}{l}-81.0 \\
-44.6\end{array}$ & -37.78 & -36.4 & -1.38 \\
\hline $\begin{array}{l}\text { sg10d_100 } \\
\text { sg10d_en100 }\end{array}$ & $\begin{array}{l}-51.74 \\
-48.75 \\
\end{array}$ & & -29.96 & & \\
\hline $\begin{array}{l}\text { sg10d_600 } \\
\text { sg10d_en600 }\end{array}$ & $\begin{array}{l}-68.75 \\
-48.86\end{array}$ & $\begin{array}{l}-69.0 \\
-46.6\end{array}$ & -19.89 & -22.4 & 2.51 \\
\hline $\begin{array}{l}\text { sg10d_600P } \\
\text { sg10d_en600P }\end{array}$ & $\begin{array}{l}-71.34 \\
-46.68\end{array}$ & $\begin{array}{l}-72.0 \\
-48.6\end{array}$ & -24.66 & -23.4 & -1.26 \\
\hline $\begin{array}{l}\text { sg12d_100 } \\
\text { sg12d_en100 }\end{array}$ & $\begin{array}{l}-53.67 \\
-65.46\end{array}$ & & 11.79 & & \\
\hline $\begin{array}{l}\text { sg12d_100P } \\
\text { sg12d_en100P }\end{array}$ & $\begin{array}{l}-50.81 \\
-68.07 \\
\end{array}$ & & 17.25 & & \\
\hline $\begin{array}{l}\text { sg13d_100 } \\
\text { sg13d_en100 }\end{array}$ & $\begin{array}{l}-53.09 \\
-39.56\end{array}$ & & -13.53 & & \\
\hline $\begin{array}{l}\text { sg13d_100P } \\
\text { sg13d_en100P }\end{array}$ & $\begin{array}{l}-49.18 \\
-34.94 \\
\end{array}$ & & -14.24 & & \\
\hline $\begin{array}{l}\text { mps10d_100 } \\
\text { mps10d_en100 }\end{array}$ & $\begin{array}{l}-53.44 \\
-48.97 \\
\end{array}$ & & -4.47 & & \\
\hline $\begin{array}{l}\text { mps12d_100 } \\
\text { mps12d_en100 }\end{array}$ & $\begin{array}{l}-55.54 \\
-52.09\end{array}$ & $\begin{array}{l}-56.0 \\
-53.7\end{array}$ & -3.45 & -2.3 & -1.15 \\
\hline $\begin{array}{l}\mathrm{mps} 12 \mathrm{~d} 1100 \mathrm{P} \\
\text { mps12d_en100P }\end{array}$ & $\begin{array}{l}-54.79 \\
-48.14 \\
\end{array}$ & $\begin{array}{l}-54.0 \\
-50.7 \\
\end{array}$ & -6.65 & -3.3 & -3.35 \\
\hline $\begin{array}{l}\text { mps12d_600 } \\
\text { mps12d_en600 }\end{array}$ & $\begin{array}{l}-77.26 \\
-52.50 \\
\end{array}$ & $\begin{array}{l}-77.5 \\
-53.7 \\
\end{array}$ & -24.77 & -23.8 & -0.97 \\
\hline $\begin{array}{l}\text { mps12d_600P } \\
\text { mps12d_en600P }\end{array}$ & $\begin{array}{l}-80.18 \\
-48.46 \\
\end{array}$ & $\begin{array}{l}-80.5 \\
-50.7 \\
\end{array}$ & -31.72 & -29.8 & -1.92 \\
\hline $\begin{array}{l}\text { mps13d_100 } \\
\text { mps13d_en100 }\end{array}$ & $\begin{array}{l}-52.33 \\
-40.45 \\
\end{array}$ & & -11.88 & & \\
\hline $\begin{array}{l}\text { mps13d_100P } \\
\text { mps13d_en100P }\end{array}$ & $\begin{array}{l}-51.14 \\
-34.96 \\
\end{array}$ & & -16.18 & & \\
\hline $\begin{array}{l}\text { mps3_600 } \\
\text { mps3_en600 }\end{array}$ & $\begin{array}{l}-82.31 \\
-57.53 \\
\end{array}$ & $\begin{array}{l}-84.3 \\
-58.3 \\
\end{array}$ & -24.78 & -26.0 & 1.22 \\
\hline $\begin{array}{l}\text { mps3_600P } \\
\text { mps3_en600P }\end{array}$ & $\begin{array}{l}-96.29 \\
-61.26\end{array}$ & $\begin{array}{l}-88.0 \\
-62.1\end{array}$ & -35.02 & -25.9 & -9.12 \\
\hline
\end{tabular}

Table 1. The energy calculations in $\mathrm{dB} . \mathrm{X} \_100 \mathrm{P}$ is on axis energy for a piston pulse at 100 feet down range. X_en600 is the input energy for a LW pulse during the 600 foot range measurements. The result of on axis analysis by both teams is shown where available, and the difference in the right most column. 


\section{VII.2 Beam Widths}

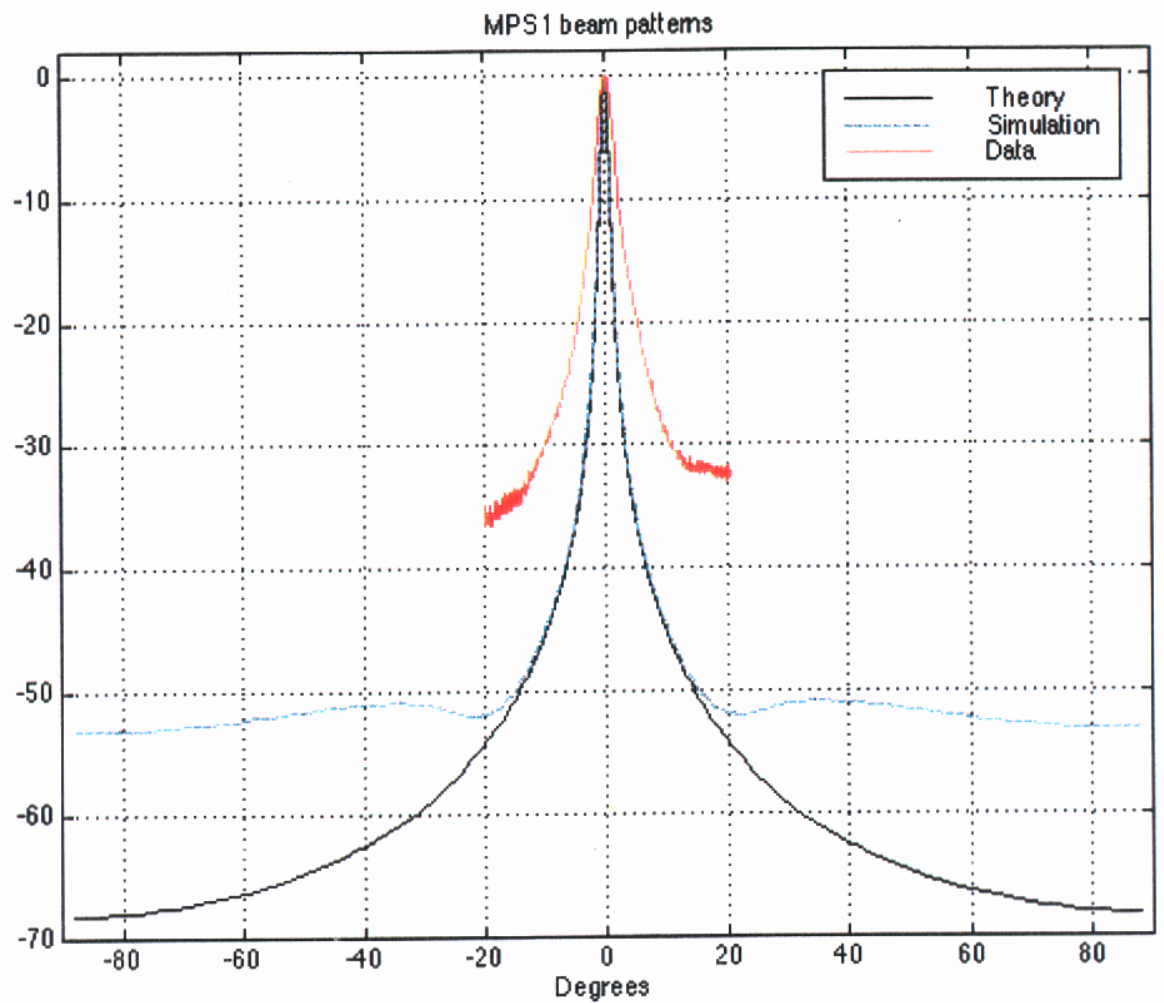

Figure 25. A beam pattern measurement for the exact theory, the 24 finite ring simulation, and the 24 ring array measured data. The two simulations differ from the data in that it was experimentally impossible to generate the high frequencies necessary for our comparison. The two simulations differ in the assumed number of signals available. For the exact theory, the source density is infinite, while the 24 ring simulation has a segmented source, resulting in higher energy far off axis. 


\begin{tabular}{|c|c|c|c|c|}
\hline $\begin{array}{c}\text { Signal } \\
\text { name }\end{array}$ & $\begin{array}{l}100 \text { feet } \\
\text { Pulse } \\
\text { (deg } \\
\text { FWHM) }\end{array}$ & $\begin{array}{l}100 \text { feet } \\
\text { Piston } \\
\text { (deg FWHM) }\end{array}$ & $\begin{array}{l}600 \text { feet } \\
\text { Pulse } \\
\text { (deg FWHM) }\end{array}$ & $\begin{array}{l}600 \text { feet } \\
\text { Piston } \\
\text { (deg FWHM) }\end{array}$ \\
\hline $\begin{array}{l}\text { tb } 20 \mathrm{kHz} \\
\text { tb } 100 \mathrm{kHz} \\
\text { tb } 240 \mathrm{kHz}\end{array}$ & & $\begin{array}{l}7.72 \\
1.52 \\
0.47\end{array}$ & & $\begin{array}{l}9.64 \\
1.59 \\
0.67\end{array}$ \\
\hline $\begin{array}{l}\mathrm{sg1}-0 \mathrm{~d} \\
\mathrm{sg} 1-2 \mathrm{~d} \\
\mathrm{sg} 1-3 \mathrm{~d}\end{array}$ & $\begin{array}{l}1.70 \\
1.45 \\
0.99\end{array}$ & $\begin{array}{l}1.38 \\
1.19\end{array}$ & 1.72 & 1.78 \\
\hline $\begin{array}{l}\text { mps1 - 0d } \\
\text { mps1 - 2d } \\
\text { mps1 - 3d }\end{array}$ & $\begin{array}{l}1.82 \\
1.15 \\
1.08\end{array}$ & $\begin{array}{l}1.31 \\
1.40\end{array}$ & 1.84 & 1.66 \\
\hline $\mathrm{mps} 3-0 \mathrm{~d}$ & & & 0.96 & 1.91 \\
\hline
\end{tabular}

Table 2. The beam width measurement, full width at half maximum in degrees. th denotes 10 cycles of the single frequency which follows, 20,100 and $240 \mathrm{kHz}$. SG1 is the Superposed Gaussian type 1, MPS1 is the Modified Pulse Spectrum type 1, MPS3 is the Modified Pulse Spectrum type 3. The compensation is noted in $\mathrm{X}-\mathrm{Nd}$ which is the measurement of signal $\mathrm{X}$ compensated to be an $\mathrm{N}$ derivative at 100 feet. The compensation is essentially no filter, for 0d, low pass, for $2 \mathrm{~d}$, and high pass, for $3 \mathrm{~d}$.

\section{Conclusions and Recommendations}

We have shown conclusively that the Localized Wave pulses can be generated and propagated in open water well past the near to far field transition point. We showed that the pulses had a very narrow beam width as expected, and no observable side lobes within measurable values, also as expected.

By the use of pulse stretching techniques, we could extend the side lobe sensitivity to more than $40 \mathrm{~dB}$, and still observed no side lobes. We were unable, due to the high error bar estimates of $+/-6 \mathrm{~dB}$, to show an improvement in relative efficiencies between the LW pulses and some of the tone bursts and between the LW pulses and their equivalent piston pulses. An improvement in the error bars, by a more controlled experiment, could clear this question up.

We found that attempting to overdrive the electronics and/or array led to a degradation of the beam both from an expected response evaluation and from an equivalent piston pulse point of view. A follow-on experiment using higher output power is called for to determine if the absence of side lobes persists as expected. Our simulation showed that we would expect small lobes and a higher overall large angle signal (Figure 25) below -50 $\mathrm{dB}$. We would also like to develop a second simulator to predict the generated fields from these arrays. Ideally it would be a classical single frequency simulator which could achieve a broad band response by superposition.

This was the second experiment using Navy grade field equipment which was able to generate the LW pulse family, and the first to propagate the pulses over appreciable distances, nearly 200 meters in this case. This experiment has shown that the Localized Wave technology is more than an interesting laboratory curiosity.

Following this successful test of the Localized Wave technology, we can recommend several additional steps which should be taken. First of all, carefully controlled experiments need to be undertaken to compare the performance of the LW pulse to its equivalent piston. This experiment showed that it is possible to generate a pulse with 
a decade of band width, and simulations show, and experiments hint, that the LW pulse performs better in terms of efficiency than its equivalent piston pulse.

A careful study should be undertaken to verify the predictions of higher efficiency for the predominant frequency of the array as detailed in Appendix A. We note that the argument detailed in Appendix A does not apply to the measurements of the voltage or power transfer functions, shown respectively in Figures 7 and 9 . It may nevertheless explain the apparent high efficiency of the $100 \mathrm{kHz}$ tone bursts relative to the pulse beams.

We recommend a study be undertaken to design $\mathrm{LW}$ pulses which will improve the beam's efficiency relative to the highly efficient tone bursts. Since the LW pulse seems to be more efficient than the equivalent piston pulse, and both seem, in general, more efficient than most other frequency tones, it may be possible to design a pulse specifically for an existing piston drive which gives greatly improved performance as well as equivalent efficiency in individual cases.

We recommend a study to determine the suitability of LW pulses for use in broad band width, covert, underwater communication. The high efficiency and lack of side lobes would seem to be an asset in shallow water, and the band width should increase throughput, even in existing communications systems.

We recommend studies to determine the suitability of the LW pulse for improving mine counter measures. The tight beam widths, lack of side lobes, increased efficiency, and broad frequency band widths of the LW pulse make it a suitable candidate for this type of location and characterization mission.

\section{Acknowledgement:}

The authors wish to thank the many people without whos help this research could not have been completed. This work was performed in part by the Lawrence Livermore National Laboratory under the auspices of the U. S. Depertment of Energy under contract no. W-7405-ENG-48. 


\section{Appendix A:}

During the course of this set of experiments, many questions were resolved, the Localized Wave propagated past the near to far field transition point for the array, the necessary signals could be generated in open water, and so on. But other questions were raised. Our beam efficiency measurements showed that apparently the LW pulse is not the most efficient beam our array could make, but that a single frequency beam at or near the maximum output frequency, the 'sweet spot' of the array, can be made more efficient. A possible reason, discovered by Henderson, is detailed below.

It should be noted that there were several problems with the data sets, caused by uncontrollable environmental factors, and that we have settled on a figure of $+/-6 \mathrm{~dB}$ error bars for each individual measurement, which means that the comparison values could have very large uncertainty. It is also true that the following argument does not apply to all of our measurements, most notably the voltage transfer function shown in figure 7 , and the voltage comparison of the expected to measured field voltage, figure 9 . The following argument applies to figure 10, the power transfer curve, and the data results in figures 16 through 21 , the comparisons of the efficiencies of the pulse beams to the tone bursts.

\section{Efficiency as a frequency-weighted average of power gain:}

Consider the electrical system consisting of the 24 ring inputs (at the transducer housing), with only one output-the output of the hydrophone and preamplifier. If the input voltages and currents are $v_{n}(t)$ and $i_{n}(t)$, respectively, for $n=1$ to 24 , and the output voltage is denoted as $v_{R}(t)$, then

$$
J^{e e}=\frac{\left.\int_{t_{0}}^{t_{0}+T} v_{R}^{2}(t)\right) d t}{\sum_{n=1}^{24} \int_{0}^{T} v_{n}(t) i_{n}(t) d t}=\frac{\int_{-\infty}^{+\infty} V_{R}(f) V_{R}^{*}(f) d f}{\sum_{n=1}^{24} \int_{-\infty}^{+\infty} V_{n}(f) I_{n}^{*}(f) d f}=\frac{\int_{0}^{+\infty} 2\left|V_{R}(f)\right|^{2} d f}{\sum_{n=1}^{24} \int_{0}^{+\infty} 2 \operatorname{Re}\left[V_{n}(f) I_{n}^{*}(f)\right] d f}
$$

where $V_{n}(f)$ and $I_{n}(f)$ are the Fourier transforms of $v_{n}(t)$ and $i_{n}(t)$ windowed over the interval $[0, T] ; V_{R}(f)$ and $I_{R}(f)$ are the Fourier transforms of $v_{R}(t)$ and $i_{R}(t)$ windowed over the interval $\left[t_{0}, t_{0}+T\right]$; and "**" denotes the complex conjugate.

Notice that the quantity

$$
G(f)=\frac{2 \operatorname{Re}\left[V_{R}(f) I_{R}^{*}(f)\right]}{\sum_{n=1}^{24} 2 \operatorname{Re}\left[V_{n}(f) I_{n}^{*}(f)\right]}=\frac{2\left|V_{R}(f)\right|^{2}}{\sum_{n=1}^{24} 2 \operatorname{Re}\left[V_{n}(f) I_{n}^{*}(f)\right]}
$$

is the power gain of our electrical system for a sinusoidal input at frequency $f$. (The sinusoid contains both positive and negative frequencies, of course. Admittedly, the power gain is here computed using finite energy signals that have been windowed to exclude the effects of reflections, rather than steady state signals. Thus, $G(f)$ is really the power gain that would be measured for a sinusoid at frequency $f$ in the absence of reflecting boundaries (assuming a nominal one Ohm load at the hydrophone preamplifier output). Furthermore, in this interpretation of the physical meaning of $G(f)$ the 24 sinusoidal voltage inputs do not usually have the same amplitude and phase. Instead, their amplitudes and phases must conform to those of $\left\{V_{n}(f): n=1\right.$ to 24$\}$ at the frequency in question.)

\section{It follows that}




$$
J^{e e}=\frac{\int_{0}^{+\infty}\left\{\sum_{n=1}^{24} 2 \operatorname{Re}\left[V_{n}(f) I_{n}^{*}(f)\right]\right\} G(f) d f}{\sum_{n=1}^{24} \int_{0}^{+\infty} 2 \operatorname{Re}\left[V_{n}(f) I_{n}^{*}(f)\right] d f}=\int_{0}^{+\infty} \mu(f) G(f) d f
$$

where

$$
\mu(f)=\frac{\sum_{n=1}^{24} 2 \operatorname{Re}\left[V_{n}(f) I_{n}^{*}(f)\right]}{\int_{0}^{+\infty}\left\{\sum_{n=1}^{24} 2 \operatorname{Re}\left[V_{n}(f) I_{n}^{*}(f)\right]\right\} d f} .
$$

Notice that $\mu(f)$ is of the form of a probability density function; i.e., it is nonnegative and has a unit-valued integral. Then the electrical efficiency $J^{e e}$ is a weighted average of the frequency-dependent power gain $G(f)$. This means that $G_{\min } \leq J^{e e} \leq G_{\max }$. Furthermore, suppose $f_{\max }$ denotes the frequency where $G(f)$ attains its maximum value, and suppose that we insert narrow band pass filters in all the inputs, each with a transfer function

$$
H(f)=\left\{\begin{array}{l}
1 \text { for }|| f\left|-f_{\max }\right|<W / 2 \\
0 \text { elsewhere }
\end{array}\right.
$$

Then with this modification the efficiency is

$$
J_{\text {mod }}^{e e}=\frac{\int_{0}^{+\infty}\left\{\sum_{n=1}^{24} 2 \operatorname{Re}\left[V_{n}(f) I_{n}^{*}(f)\right]\right\}|H(f)|^{2} G(f) d f}{\left.\sum_{n=1}^{24} \int_{0}^{+\infty} 2 \operatorname{Re}\left[V_{n}(f) I_{n}^{*}(f)\right] H(f)\right|^{2} d f} \approx \frac{W \times\left\{\sum_{n=1}^{24} 2 \operatorname{Re}\left[V_{n}\left(f_{\max }\right) I_{n}^{*}\left(f_{\max }\right)\right]\right\} G\left(f_{\max }\right)}{W \times \sum_{n=1}^{24} 2 \operatorname{Re}\left[V_{n}\left(f_{\max }\right) I_{n}^{*}\left(f_{\max }\right)\right]}=G\left(f_{\max }\right)
$$

and it follows that

$$
J_{\bmod }^{e e} \approx G_{\max } \geq J^{e e}
$$

The approximation becomes exact as the bandwidth $W$ approaches zero.

If the inputs are replaced by sinusoids at $f_{\max }$, whose amplitudes and phases match those of the original signals' Fourier transforms at $f=f_{\max }$, then the efficiency becomes $\mathrm{G}_{\max }$.

In terms of ability to present the maximum signal energy on axis using a given amount of energy supplied to the transducer array, localized waves cannot beat sinusoids of the sweet spot frequency $f_{\max }$. 


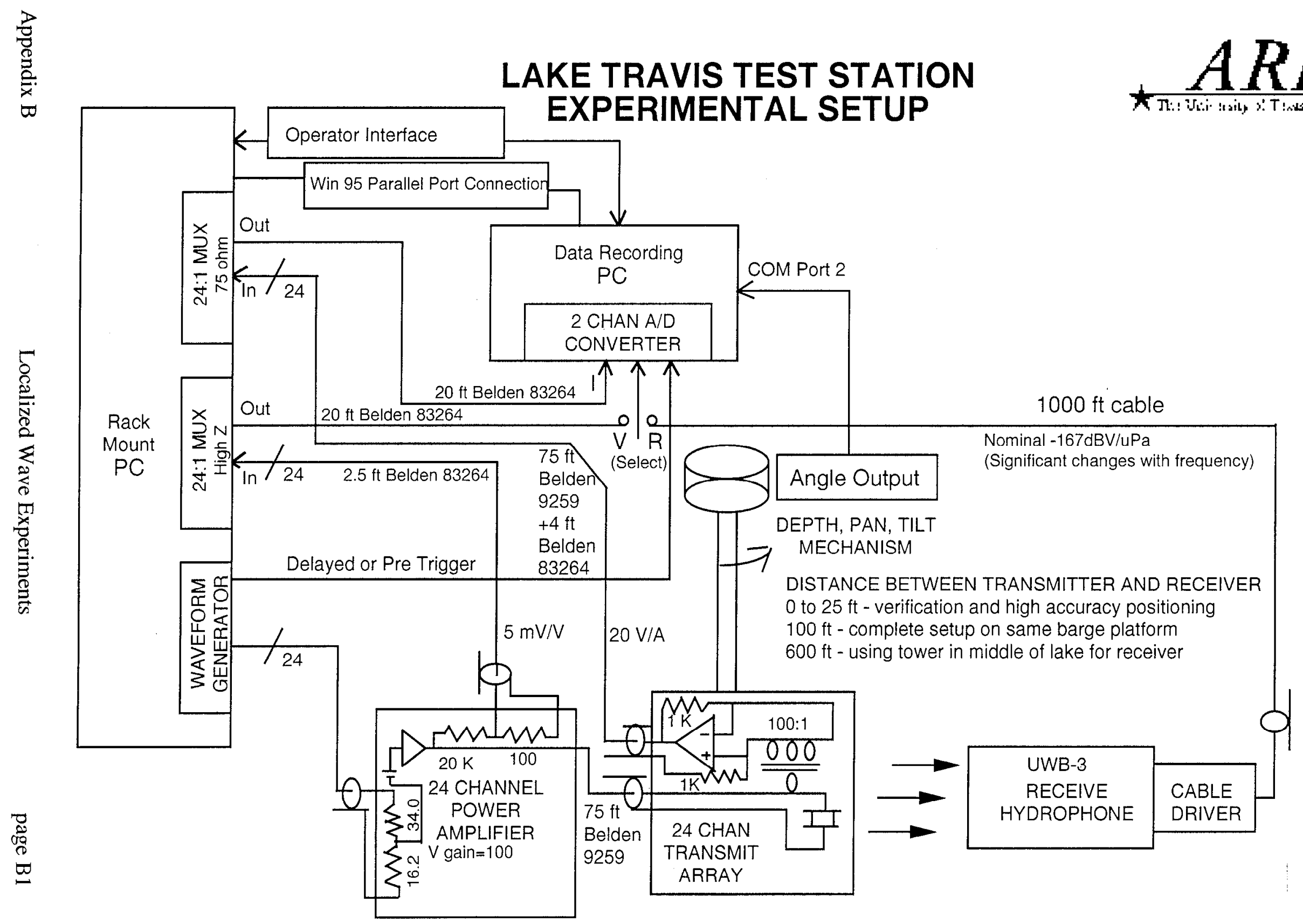




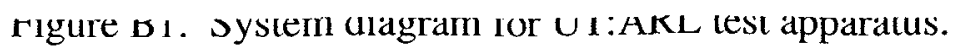



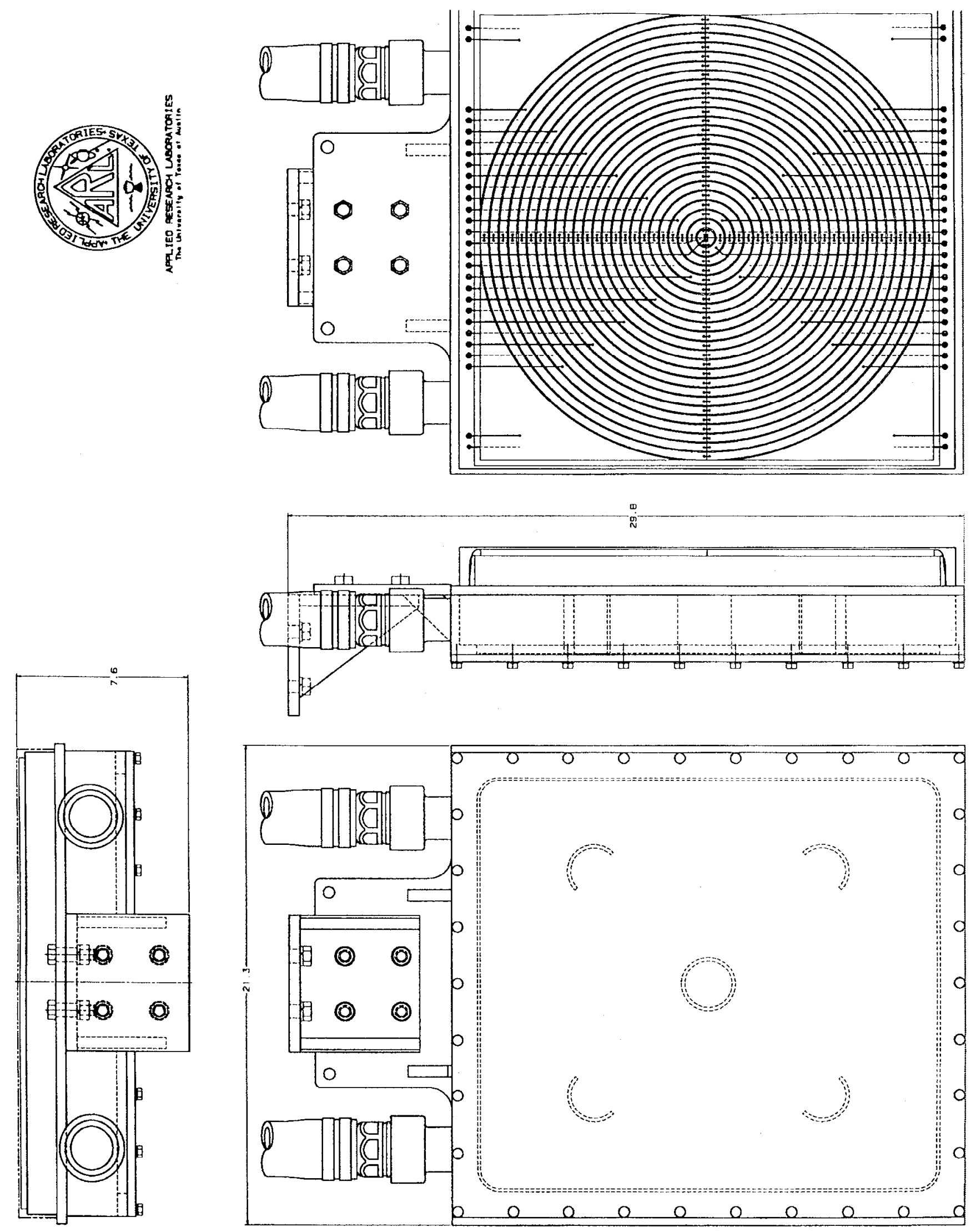

Figure B2. Wiring diagram and support braces for 24 ring individually addressable array. 

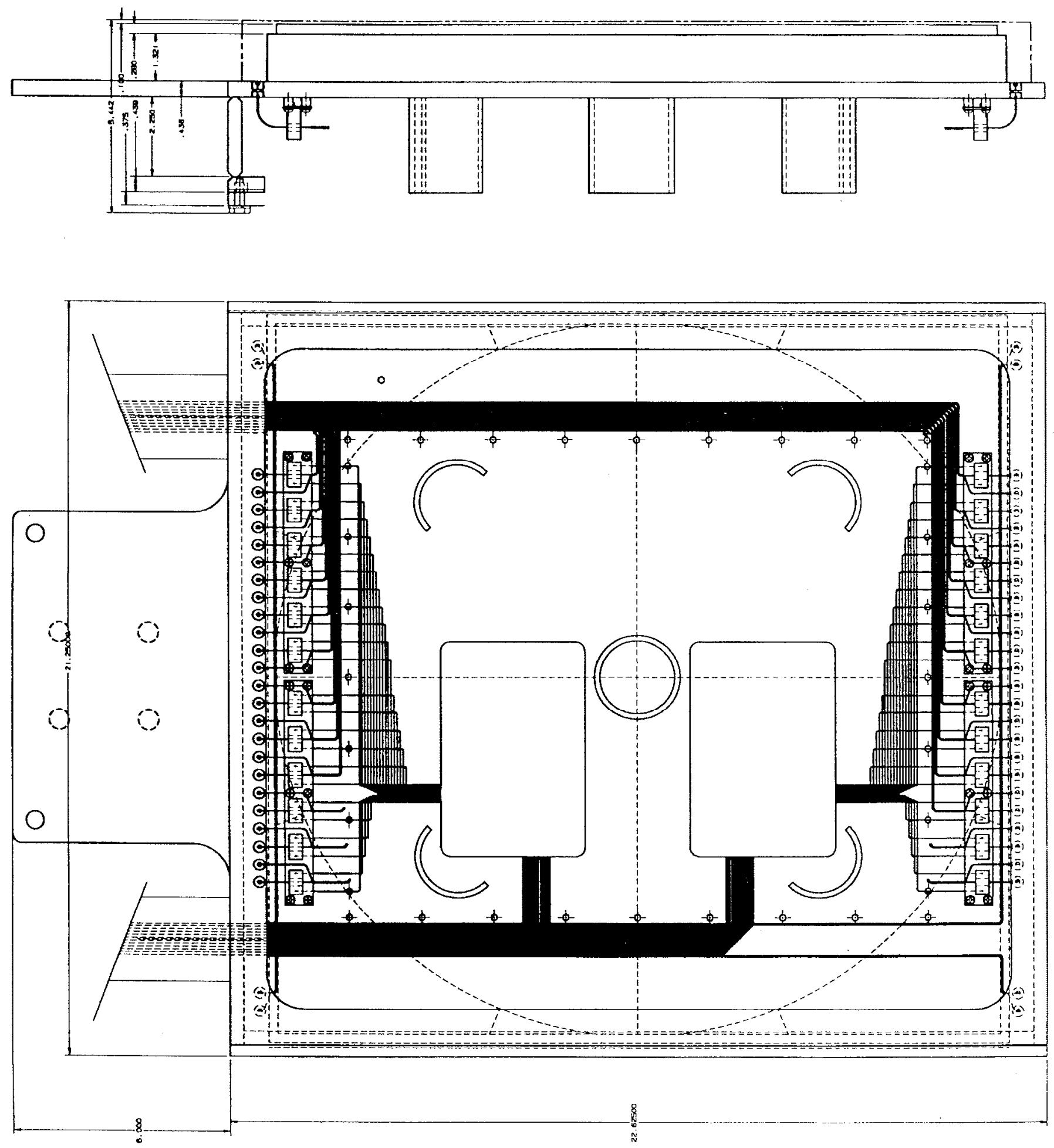

Figure B3. Wiring diagram of 24 ring individually addressable array 


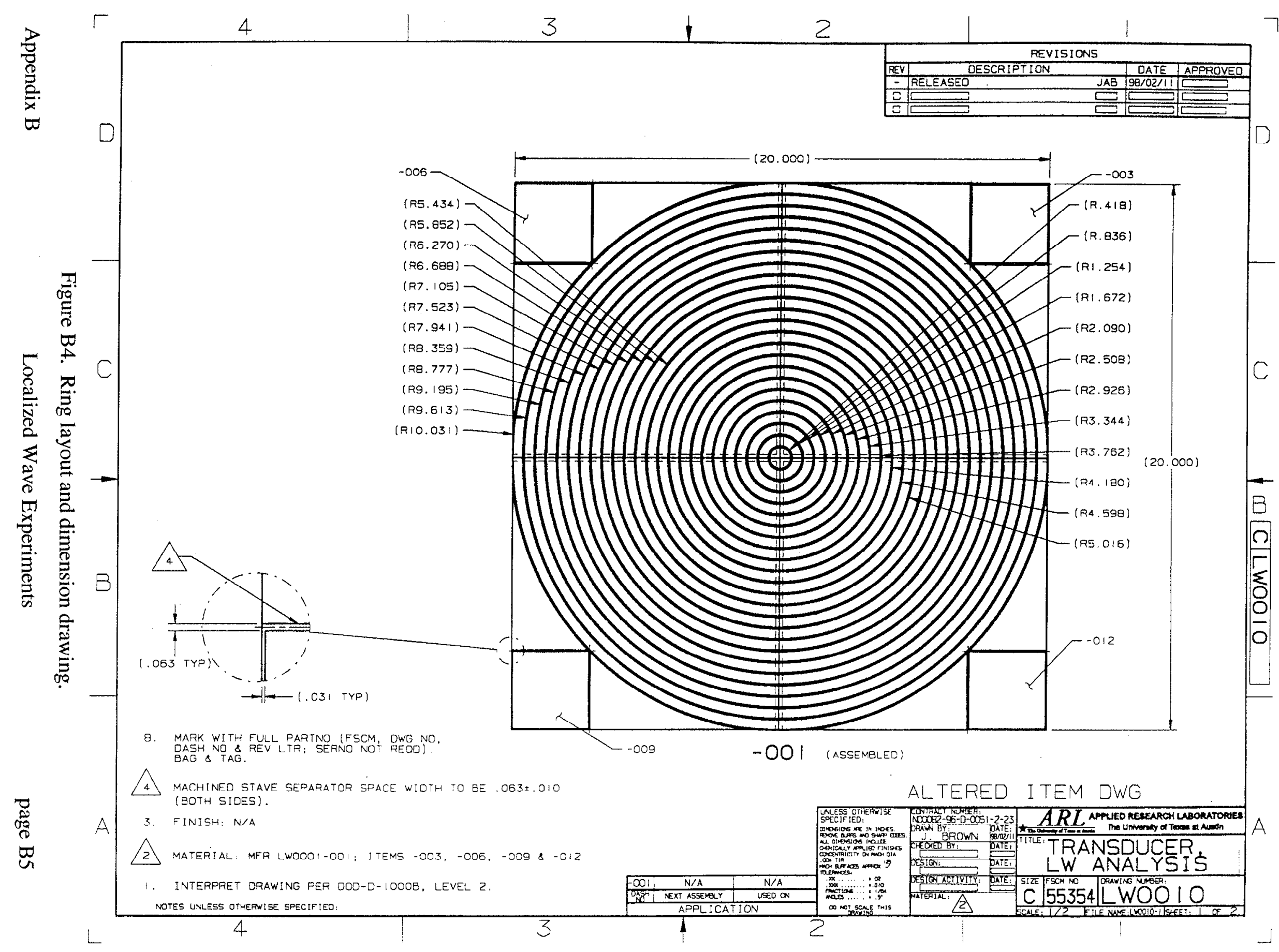




\section{Appendix C.}

On axis time signals.

During our experiments, we made an effort to precorrect the input signals to compensate for imperfections in the frequency response of the array. To do this, we used a model of the system of transducer, propagation and receiver which implied a resultant received signal, in the absence of imperfections, which would be the third derivative of the input signal. The expected signals are shown in figure $\mathrm{C} 1$.

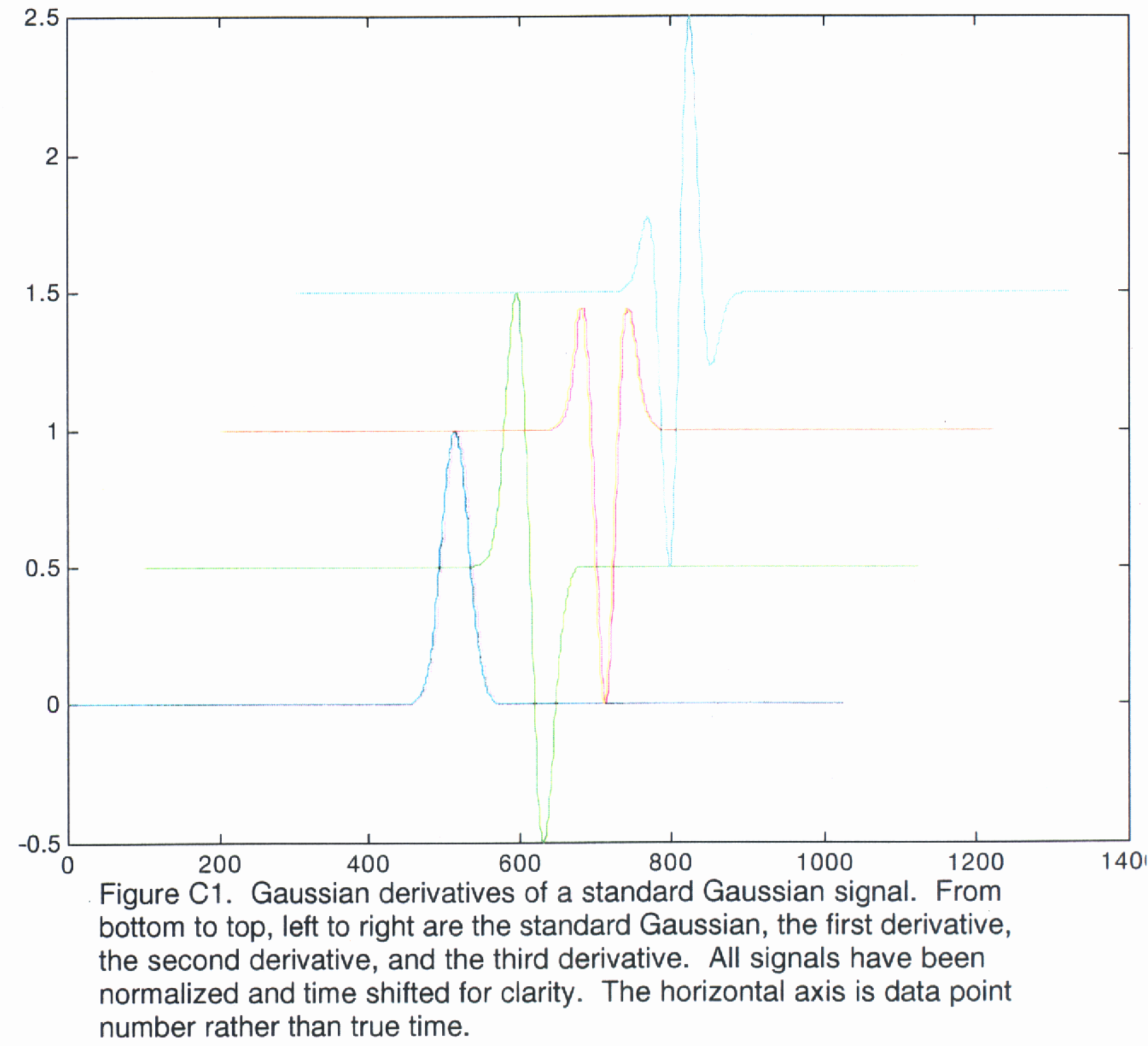

During our experiments at a 100 foot transmitter to receiver distance, we measured signals which could be interpreted as a second derivative, so we made an effort to precompensate for both an expected second and third derivative signal. We also launched signals with no compensation whatsoever, since we could not measure the amplitude and phase response of the receiver as a function of frequency. This turned 
out to be prudent, since our measurements at 600 feet seem to imply a clear third derivative, and apparently we were still in the near field of our array at 100 feet.

The following figures are of the measured voltage resulting from the launch of a Localized Wave, the equivalent piston drive for the LW pulses, and the three calibration tone bursts at 20,100 and $240 \mathrm{kHz}$. The horizontal scales are arbitrary uncorrected voltages, and the vertical scales are time.

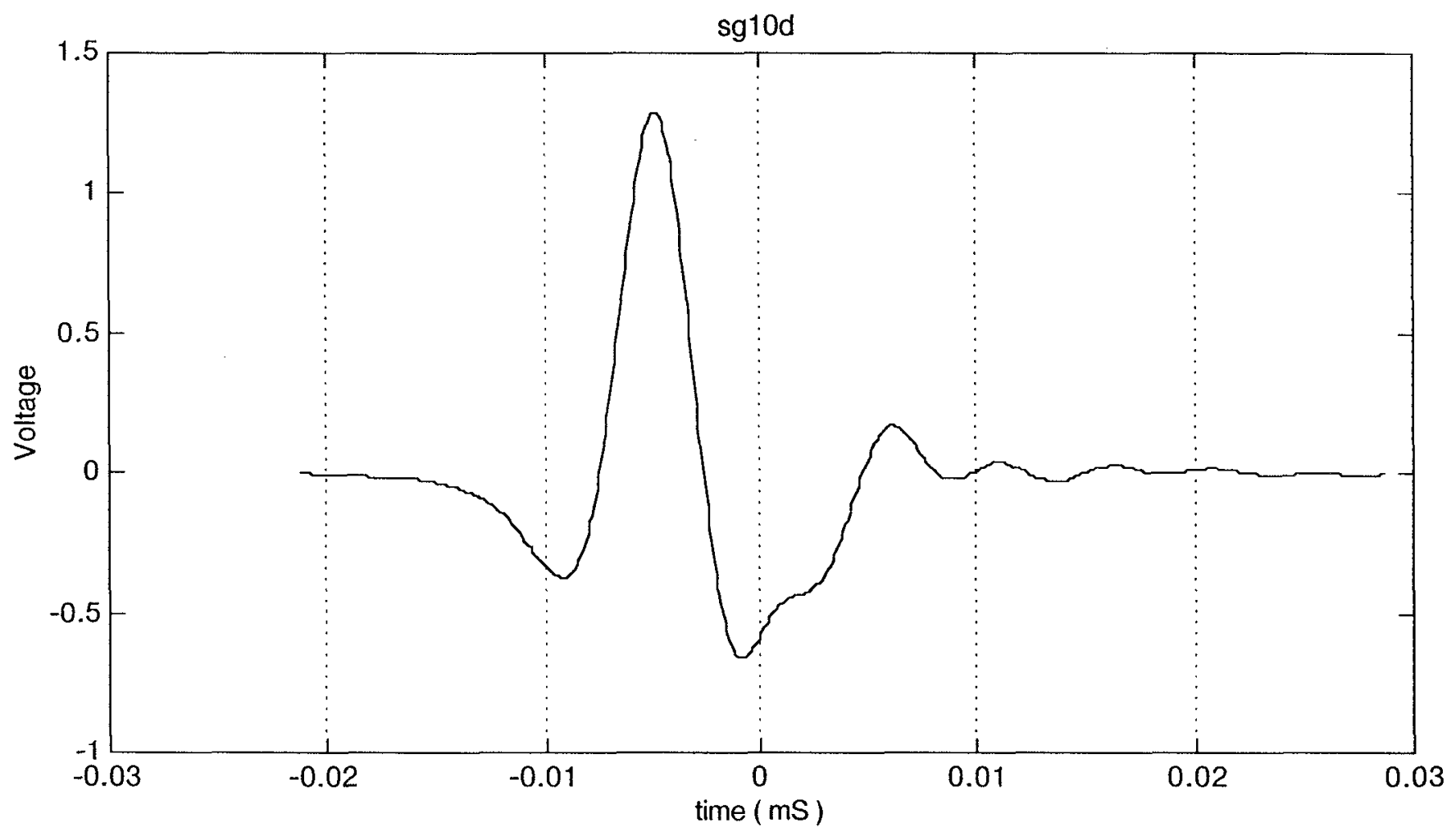

Figure C2. The received signal from an uncompensated Superposed Gaussian at 100 feet separation. 


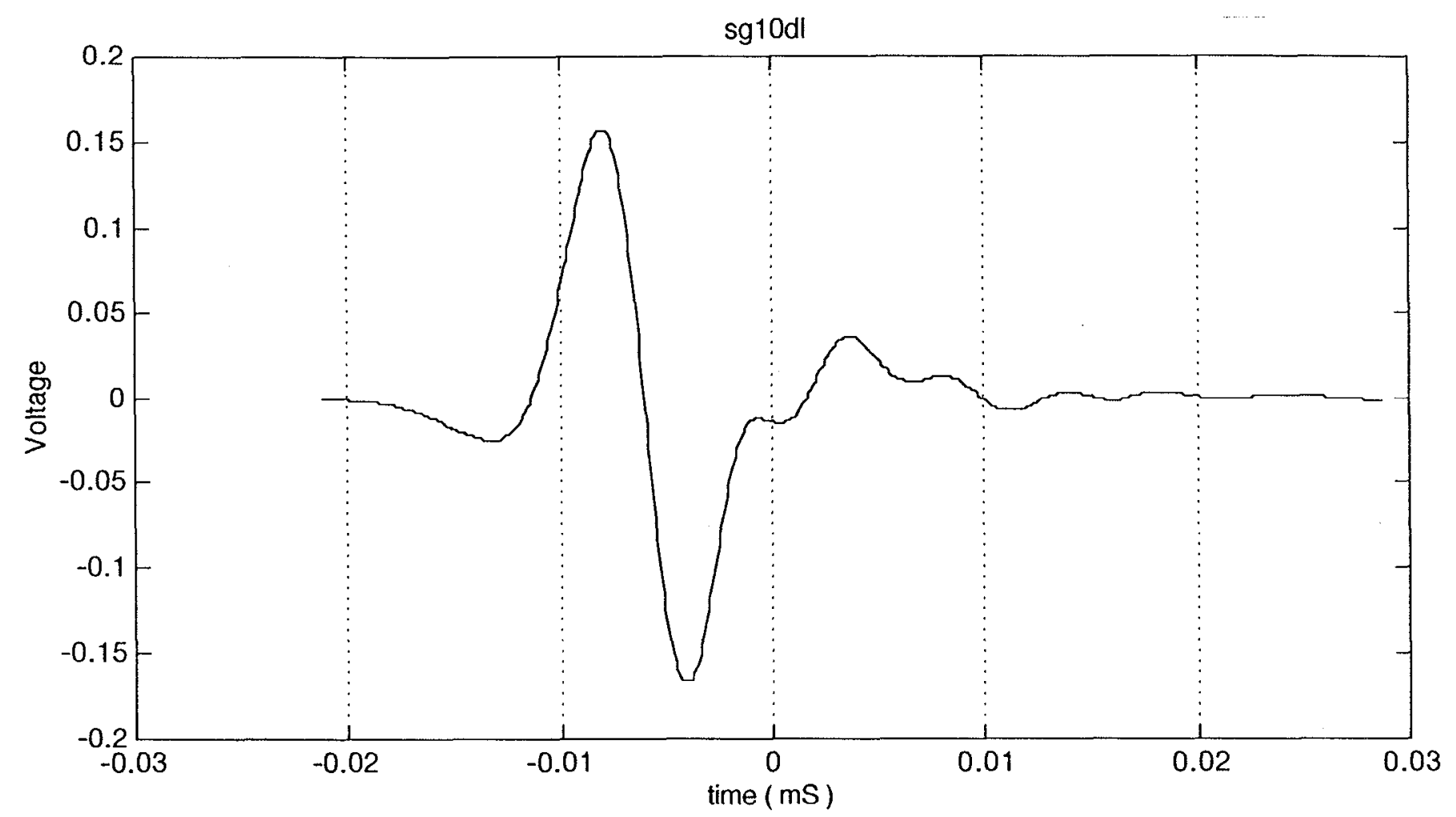

Figure C3. The on axis response for Superposed Gaussian 1 with no compensation measured at 600 feet.

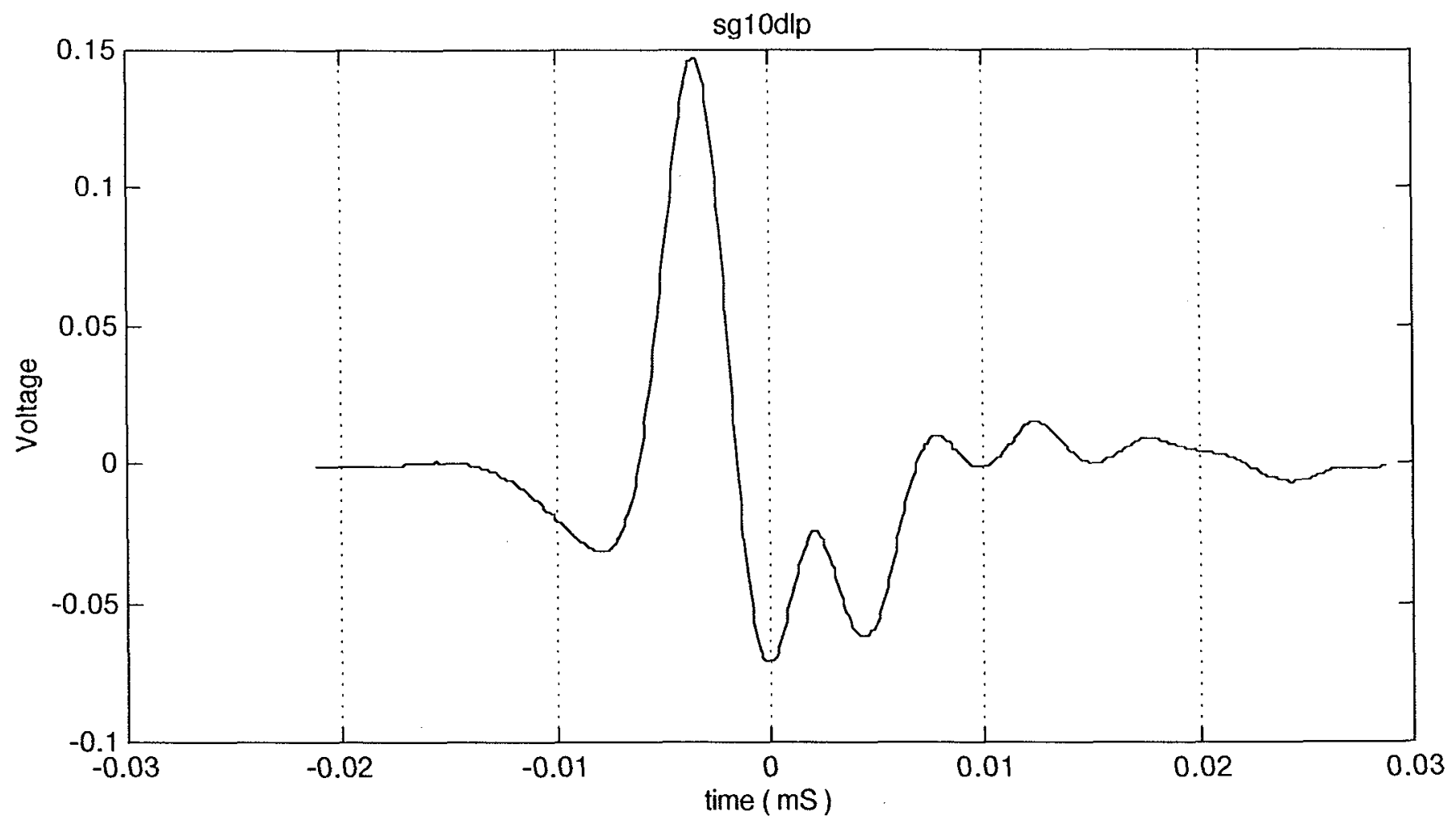

Figure C4. The on axis response for Superposed Gaussian 1 piston equivalent with no compensation measured at 600 feet. 


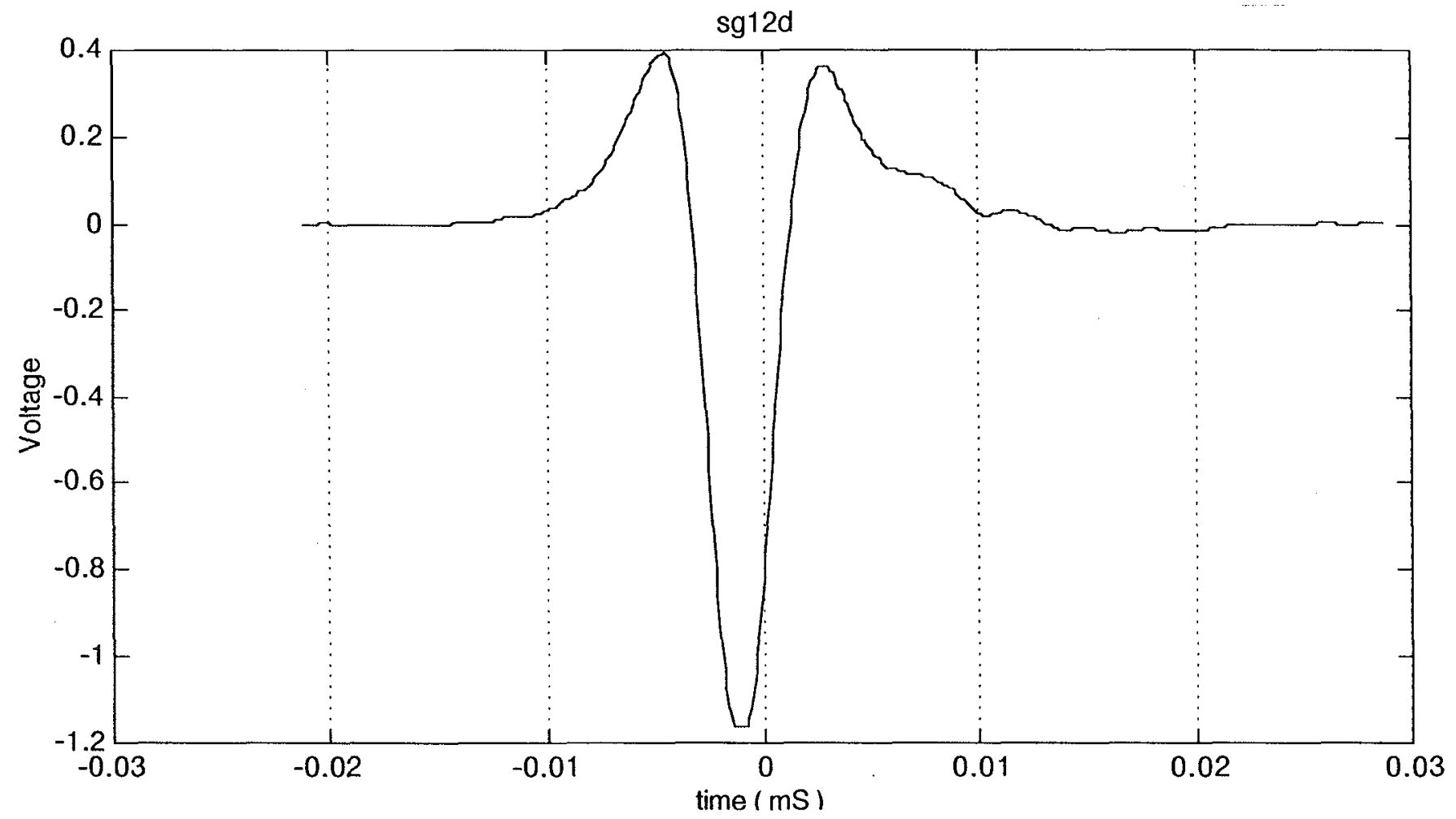

Figure C5. The received signal from a $2^{\text {nd }}$ derivative compensated Superposed Gaussian at 100 feet separation.

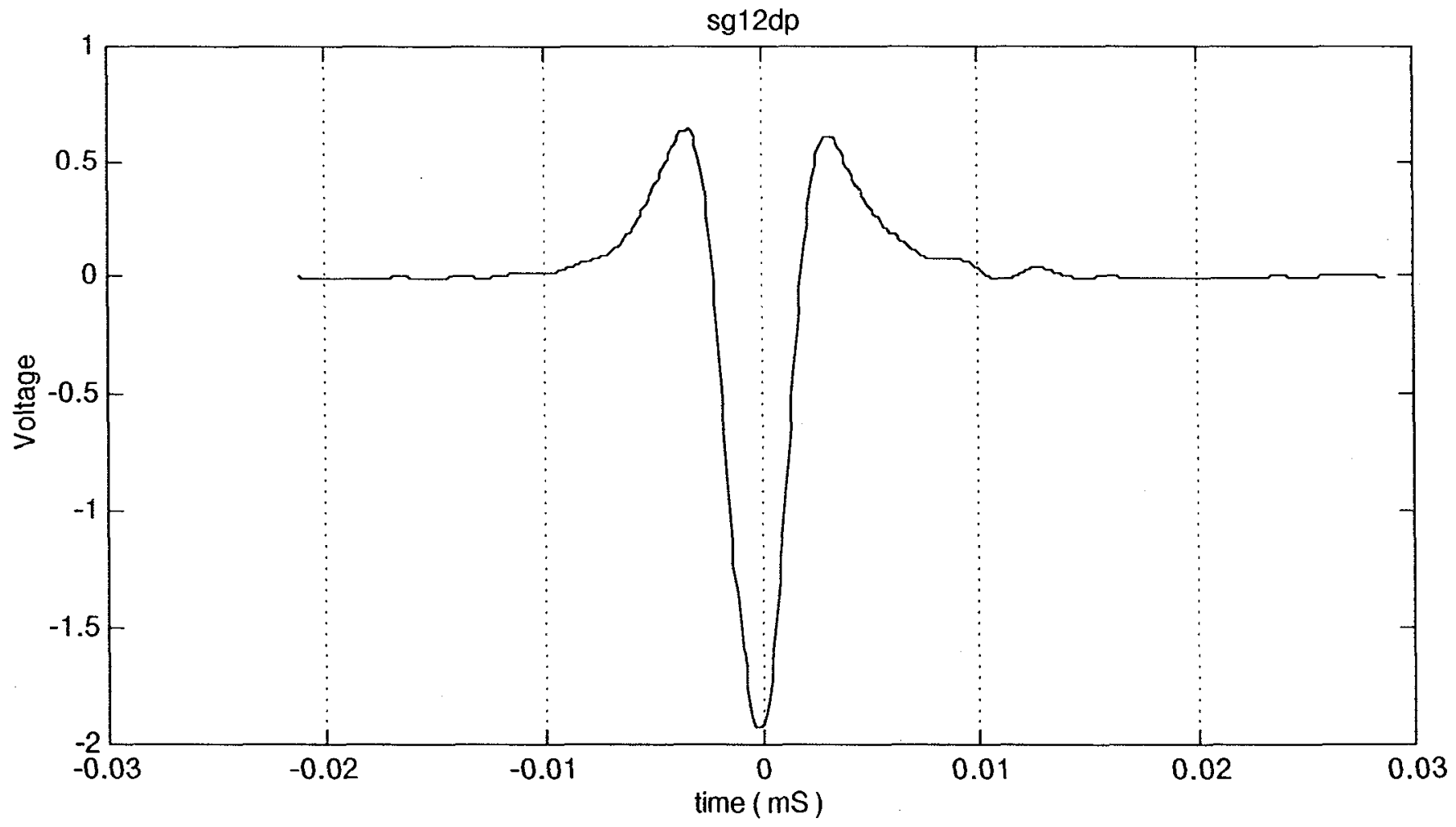

Figure C6. The received signal from an $2^{\text {nd }}$ derivative compensated Superposed Gaussian equivalent piston at 100 feet separation. 


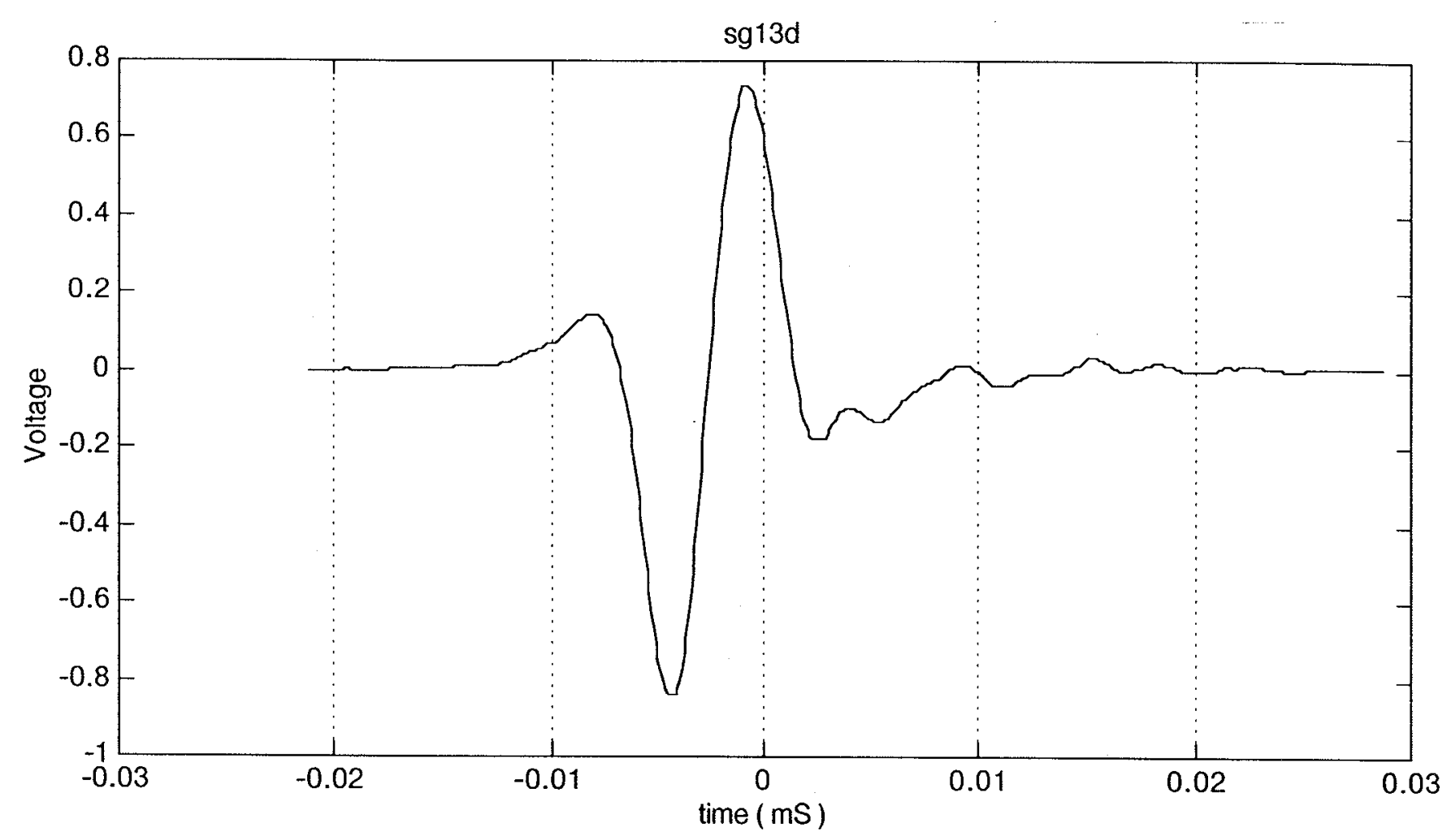

Figure C7. The received signal from a 3rd derivative compensated Superposed Gaussian at 100 feet separation.

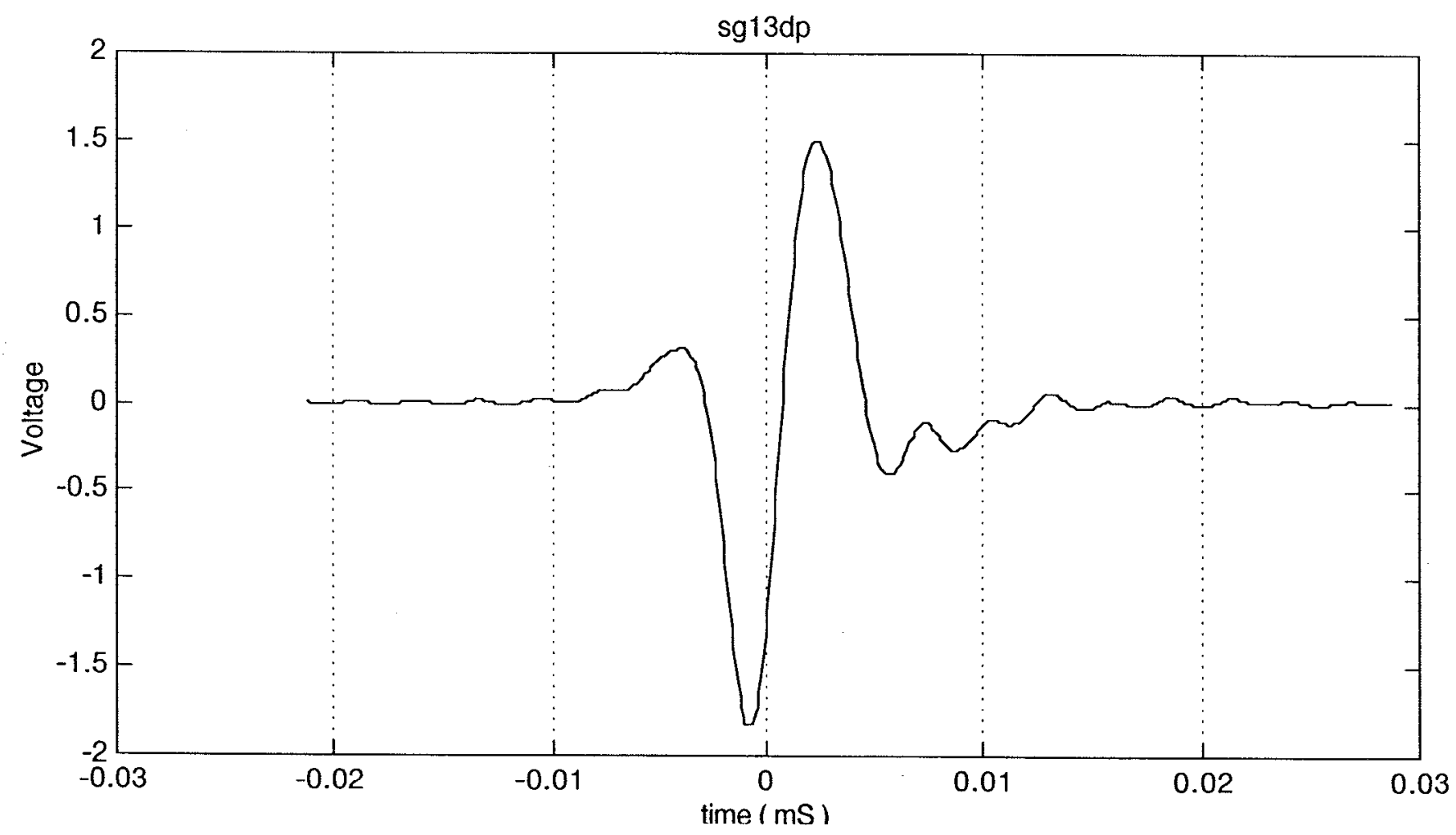

Figure C8. The received signal from a 3rd derivative compensated Superposed Gaussian equivalent piston at 100 feet separation. 


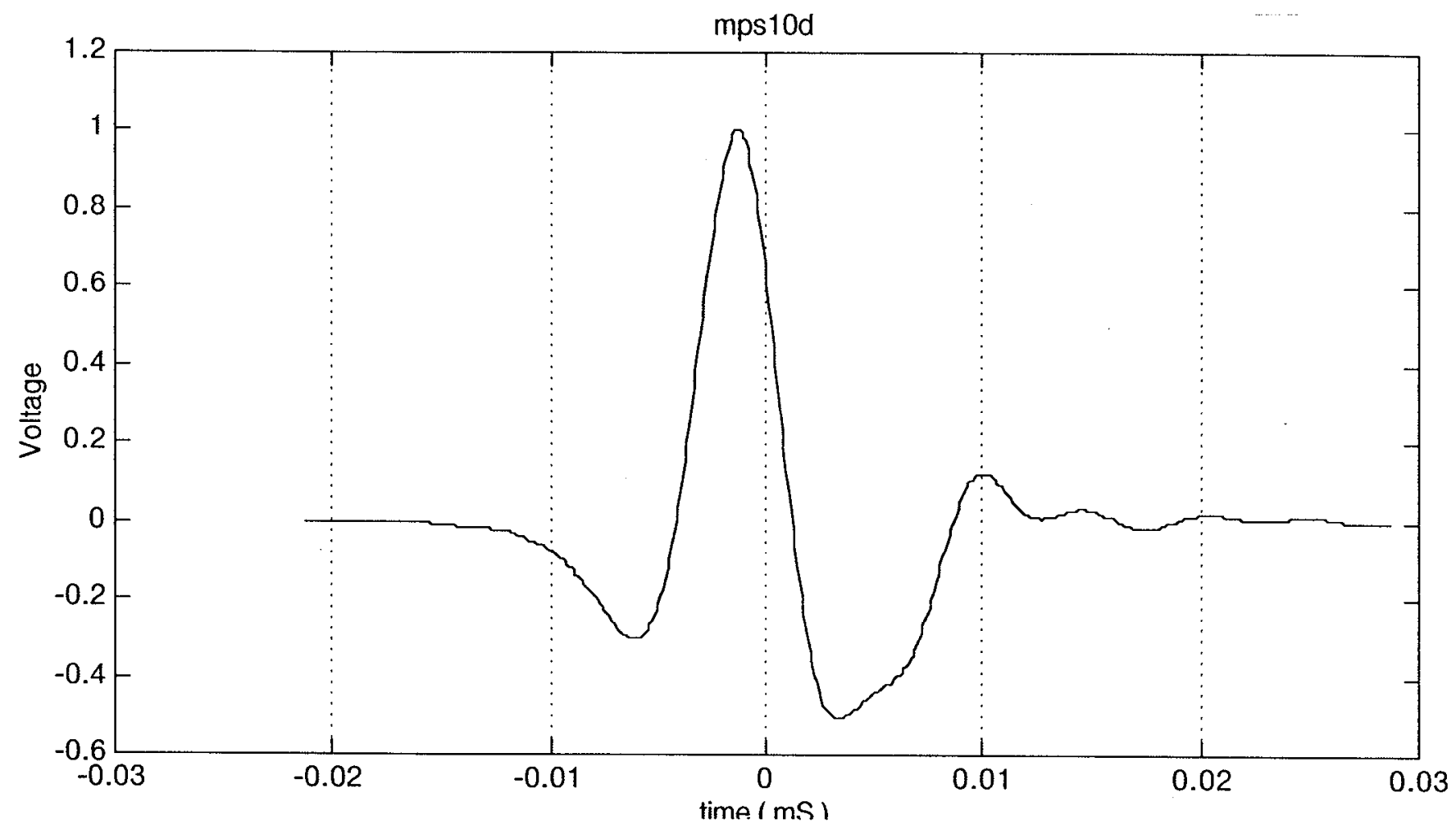

Figure C9. The received signal from an uncompensated Modified Pulse Spectrum pulse at 100 feet separation.

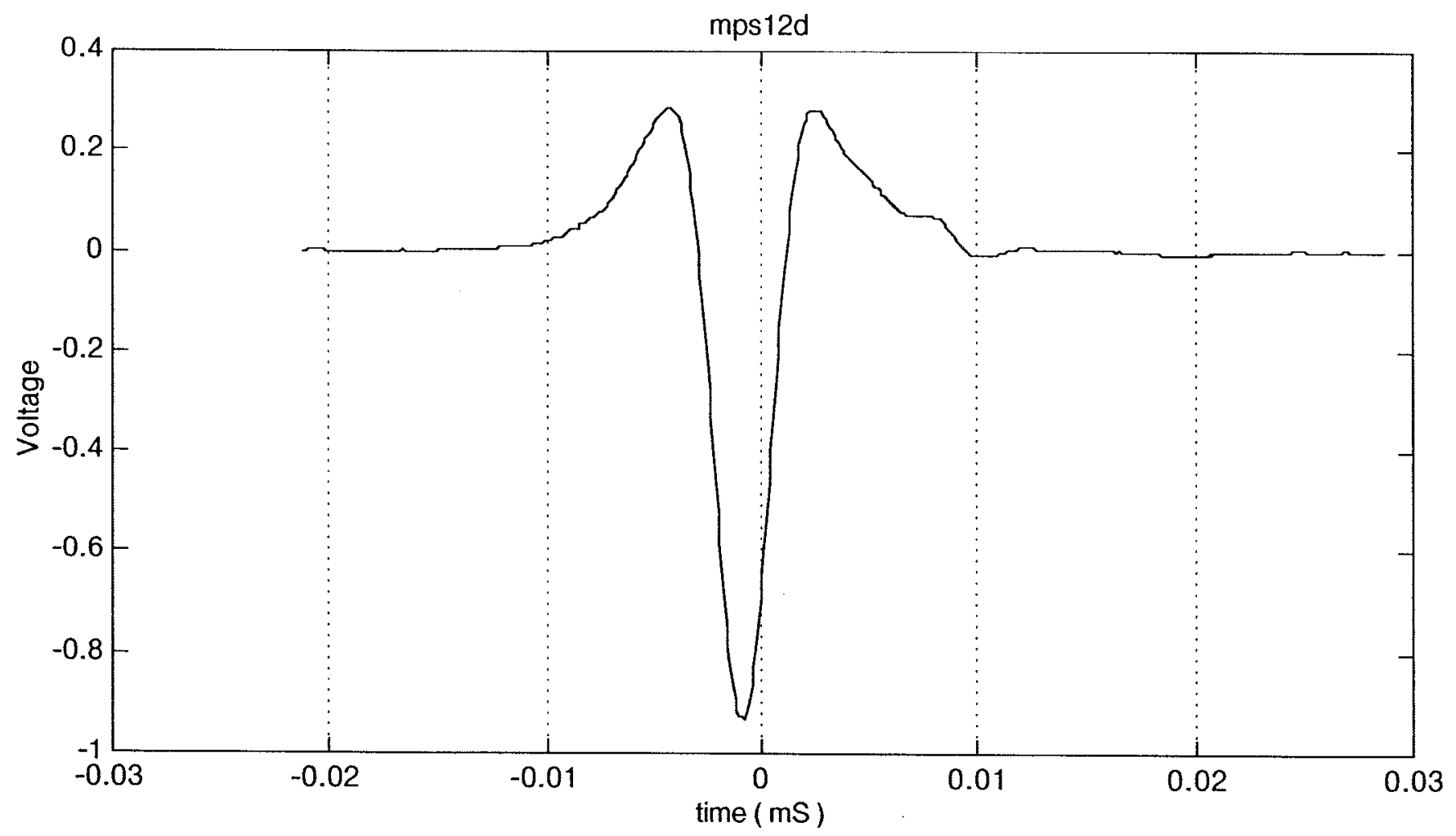

Figure $\mathrm{C} 10$. The received signal from an uncompensated Modified Pulse Spectrum pulse equivalent piston at 100 feet separation. 


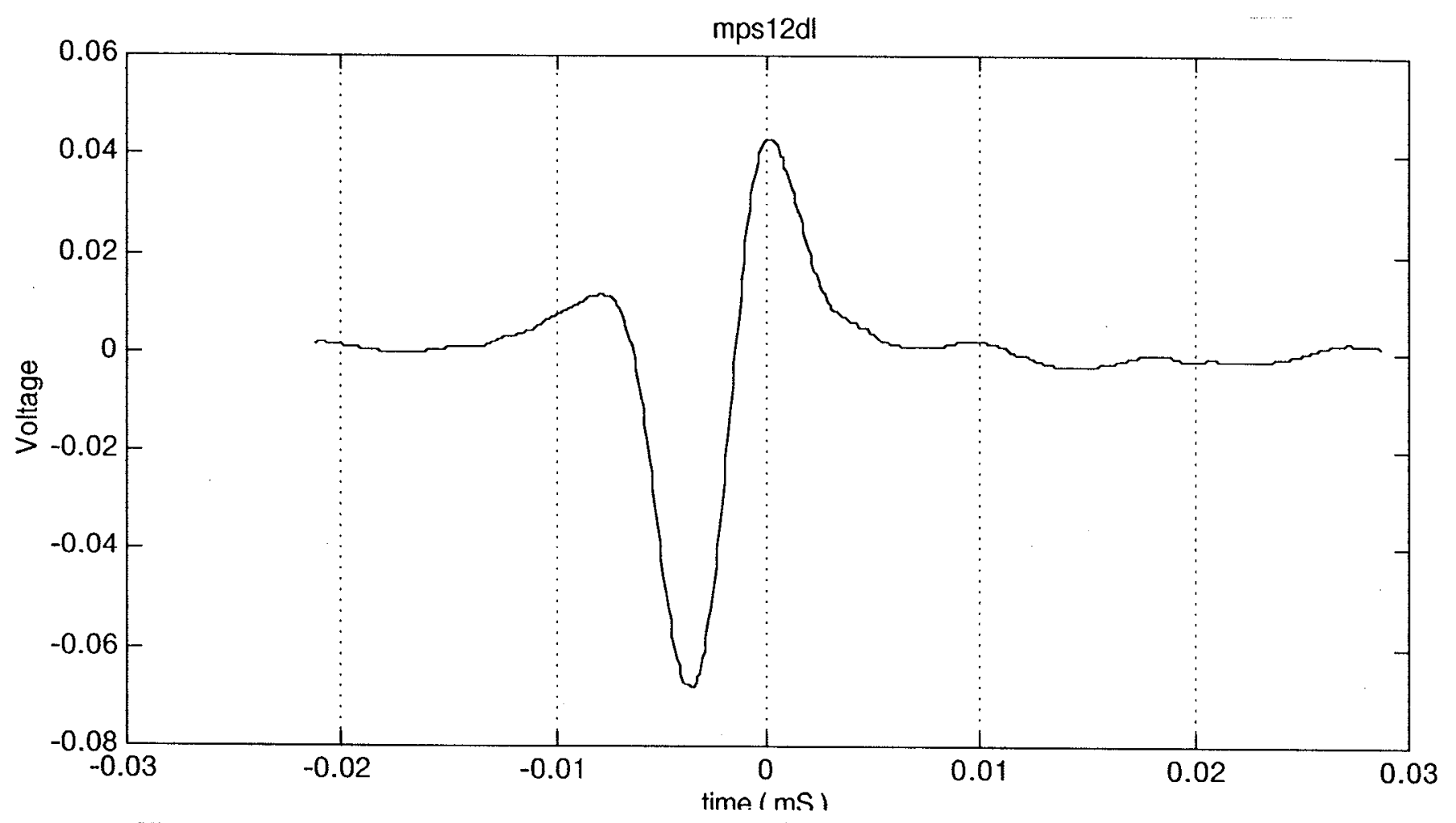

Figure $\mathrm{C} 11$. The received signal from a $2^{\text {nd }}$ derivative compensated Modified Pulse Spectrum pulse at 600 feet separation.

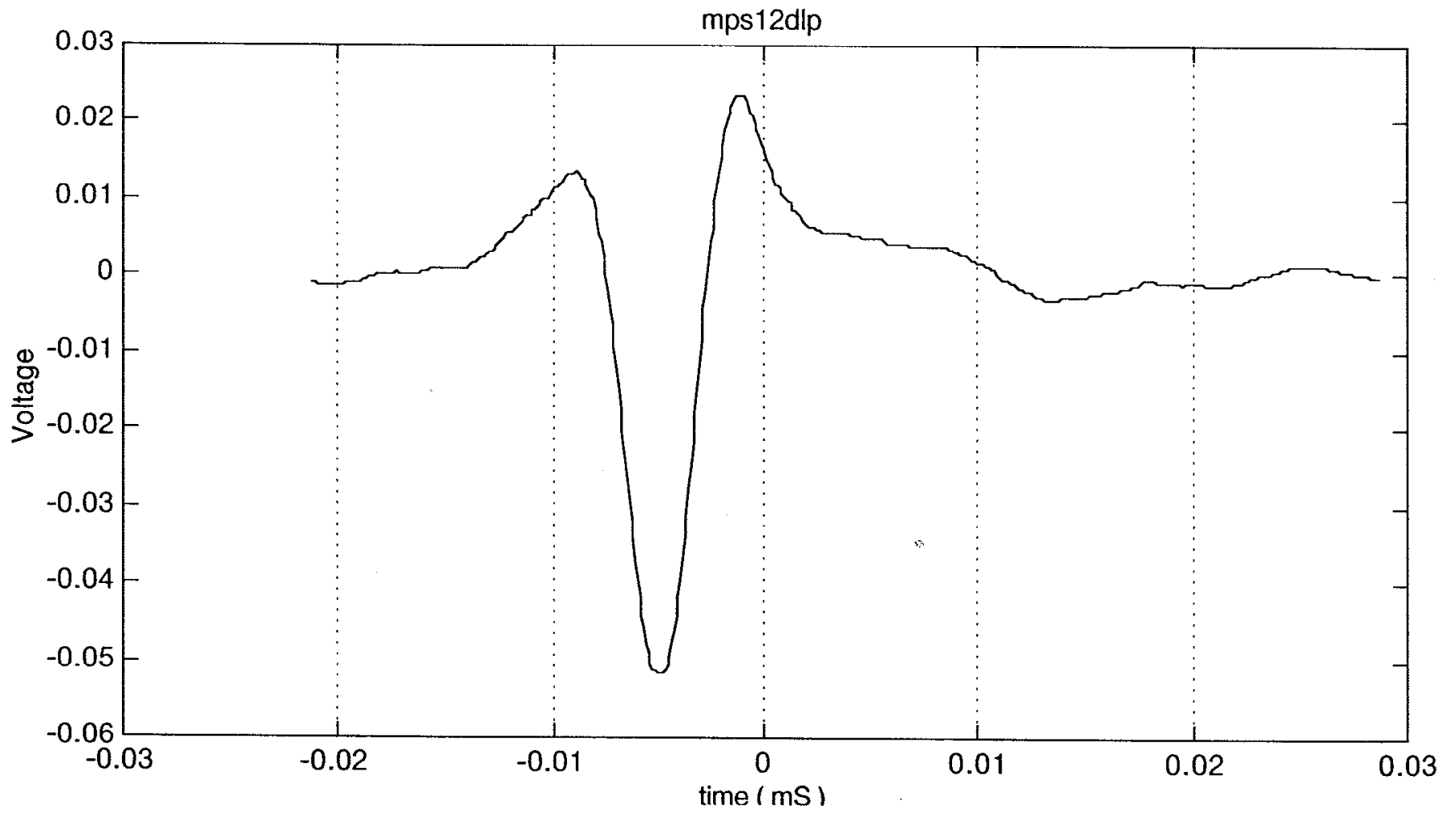

Figure $\mathrm{C} 12$. The received signal from a $2^{\text {nd }}$ derivative compensated Modified Pulse Spectrum pulse equivalent piston at 600 feet separation. 


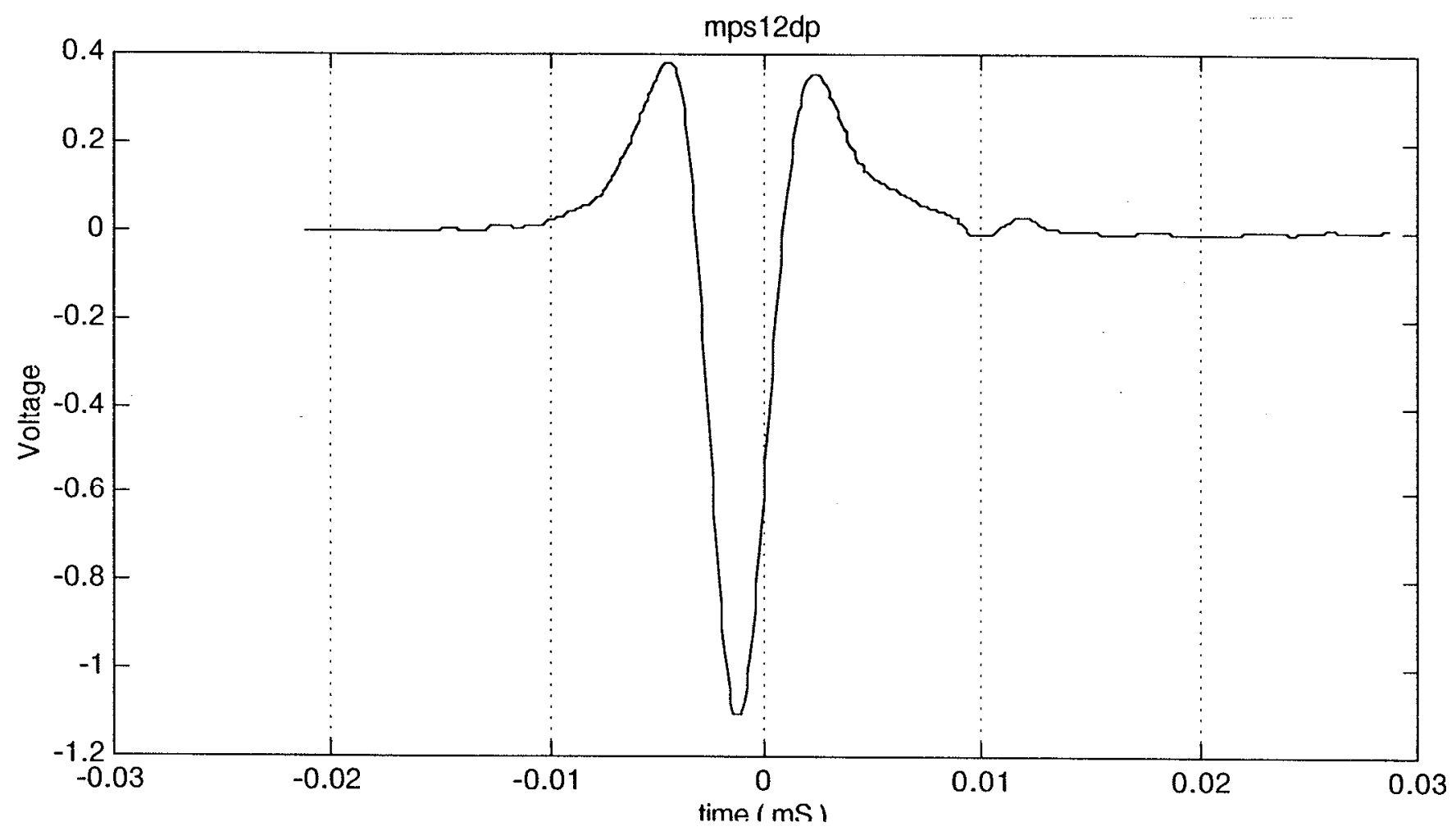

Figure $\mathrm{C} 13$. The received signal from a $2^{\text {nd }}$ derivative compensated Modified Pulse Spectrum pulse equivalent piston at 100 feet separation.

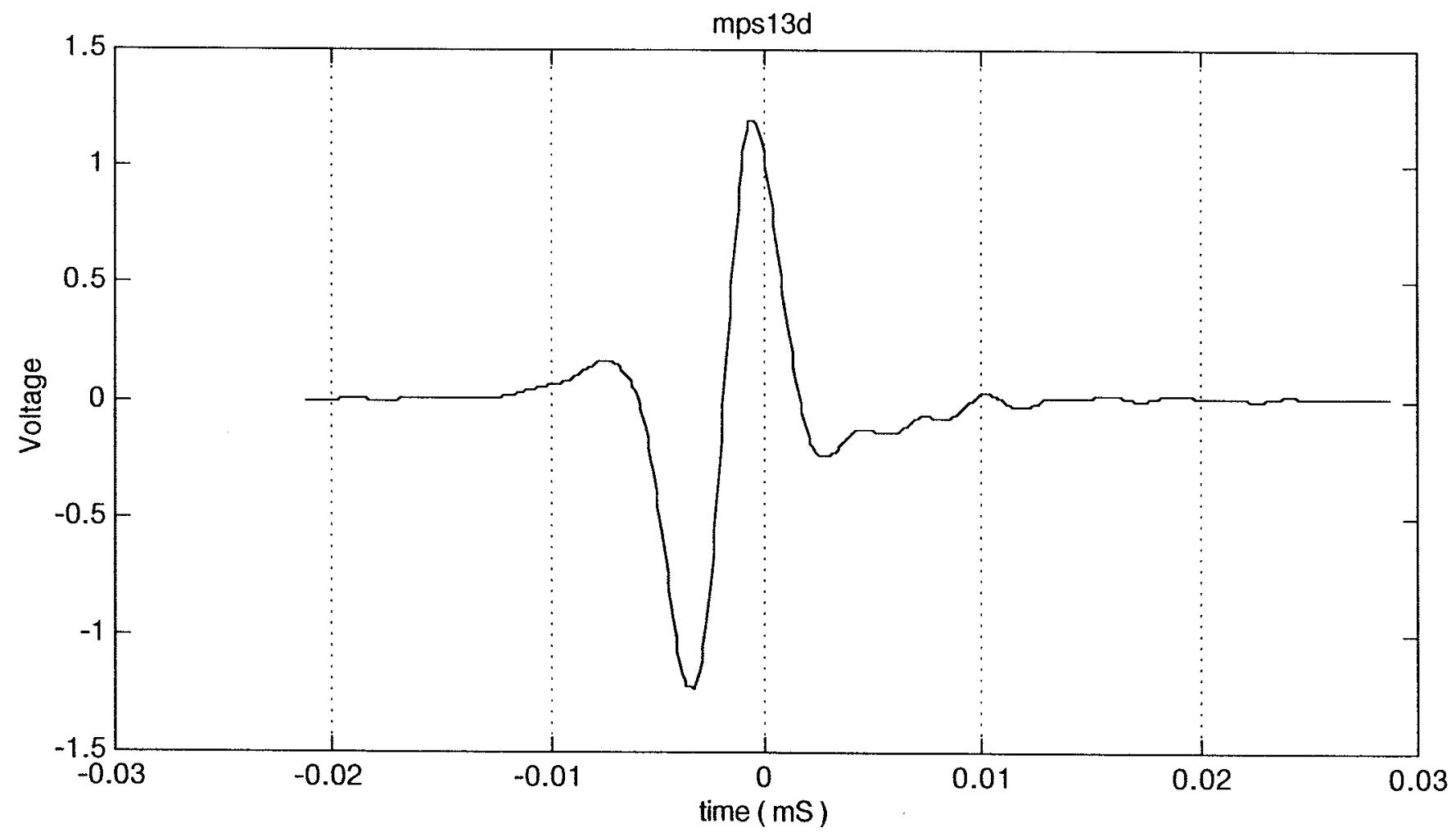

Figure $\mathrm{C} 14$. The received signal from a 3rd derivative compensated Modified Pulse Spectrum pulse equivalent piston at 100 feet separation. 


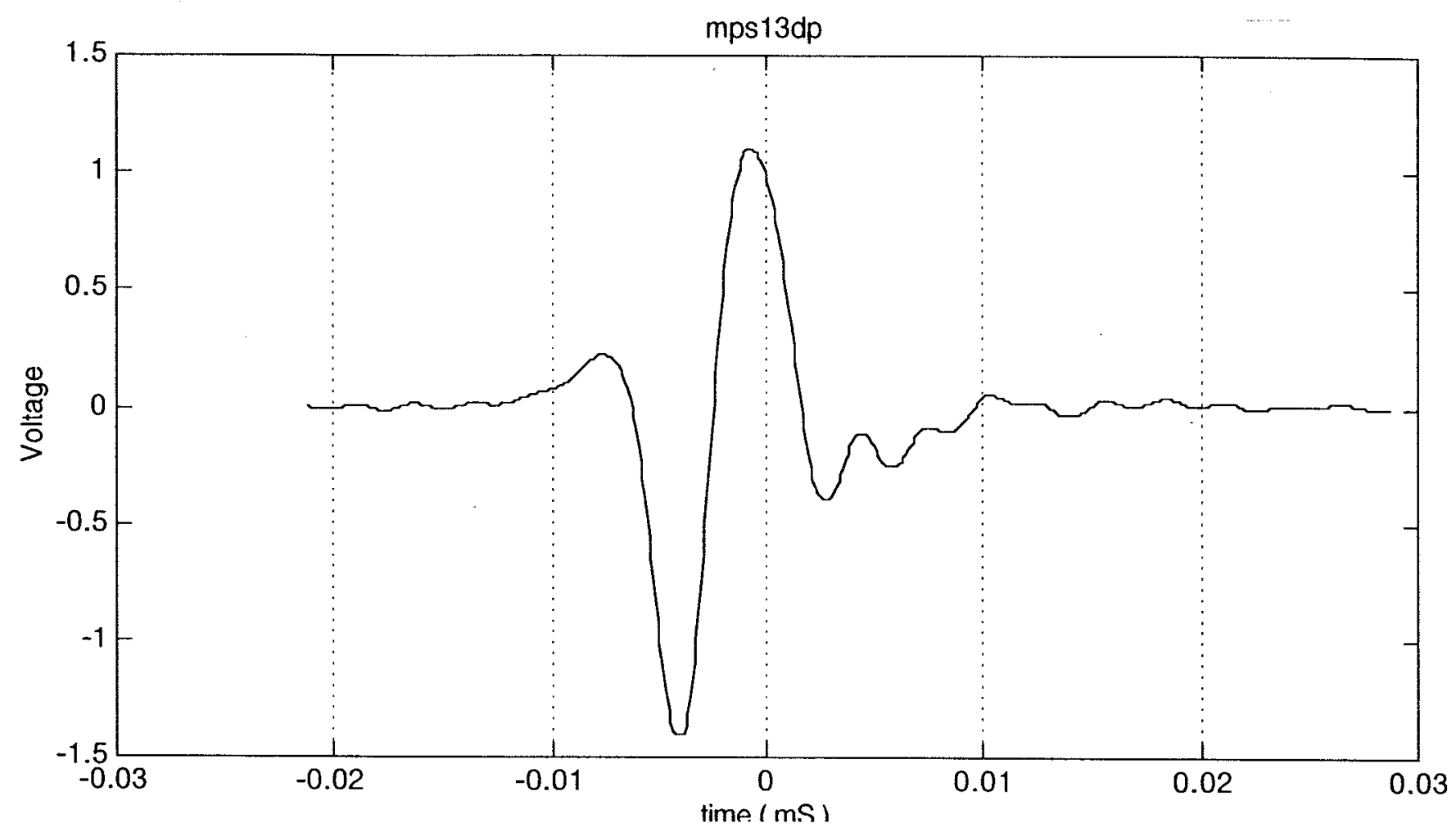

Figure $\mathrm{C} 15$. The received signal from a 3rd derivative compensated Modified Pulse Spectrum pulse equivalent piston at 100 feet separation.

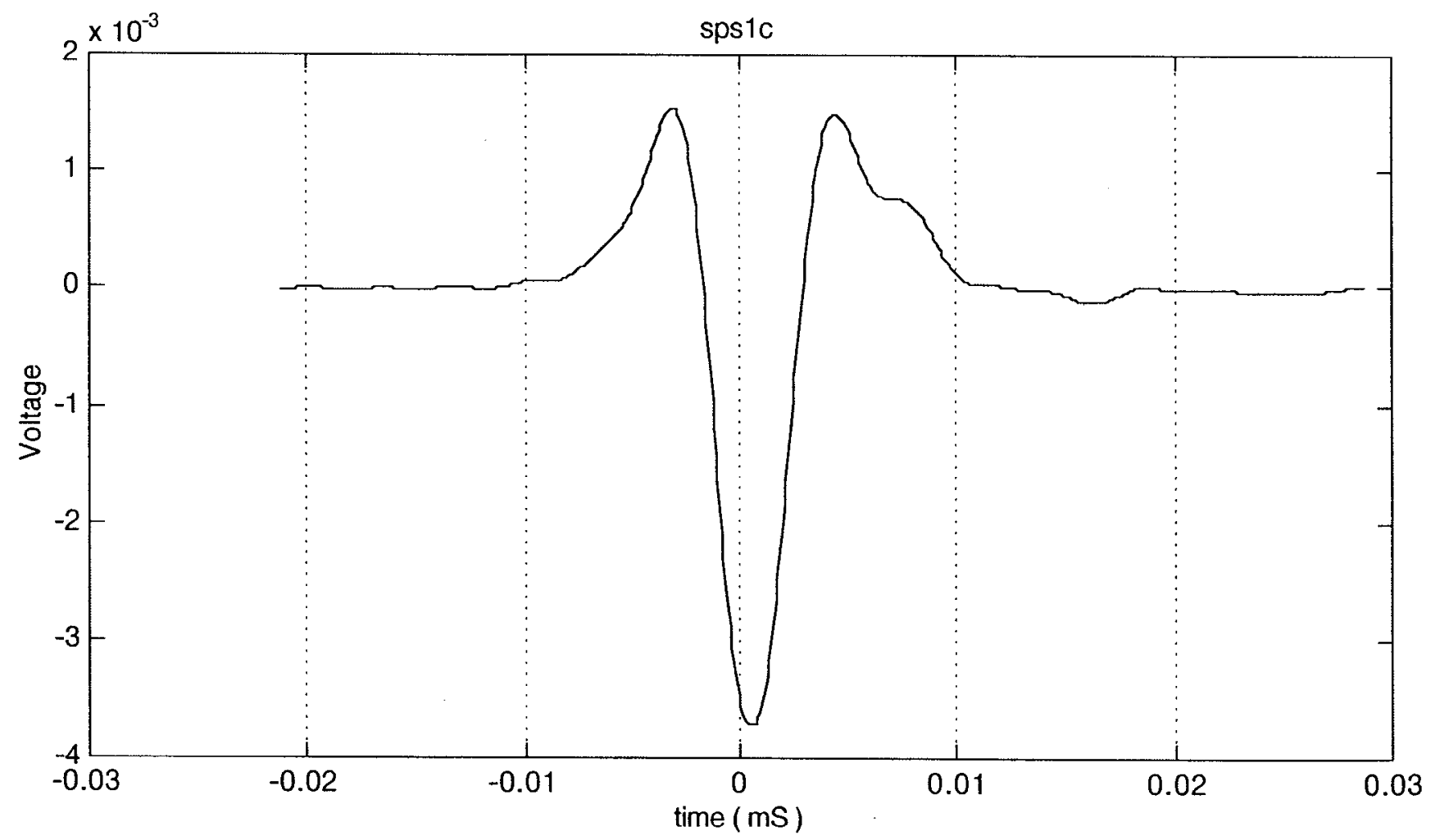

Figure C16. The received signal from a 3rd derivative compensated Superposed Gaussian pulse at 100 feet separation. 


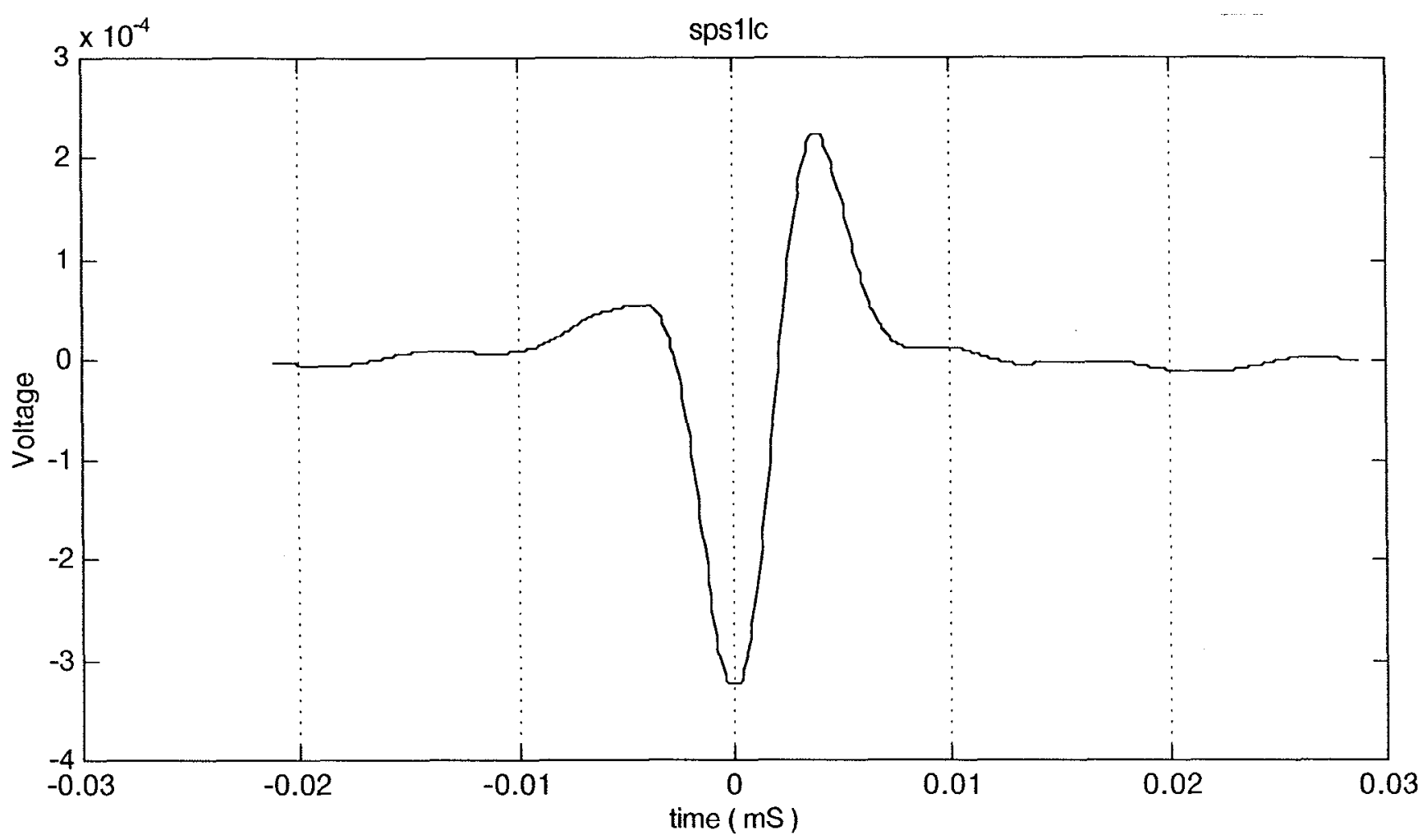

Figure C17. The received signal from an uncompensated Superposed Gaussian pulse at 600 feet separation.

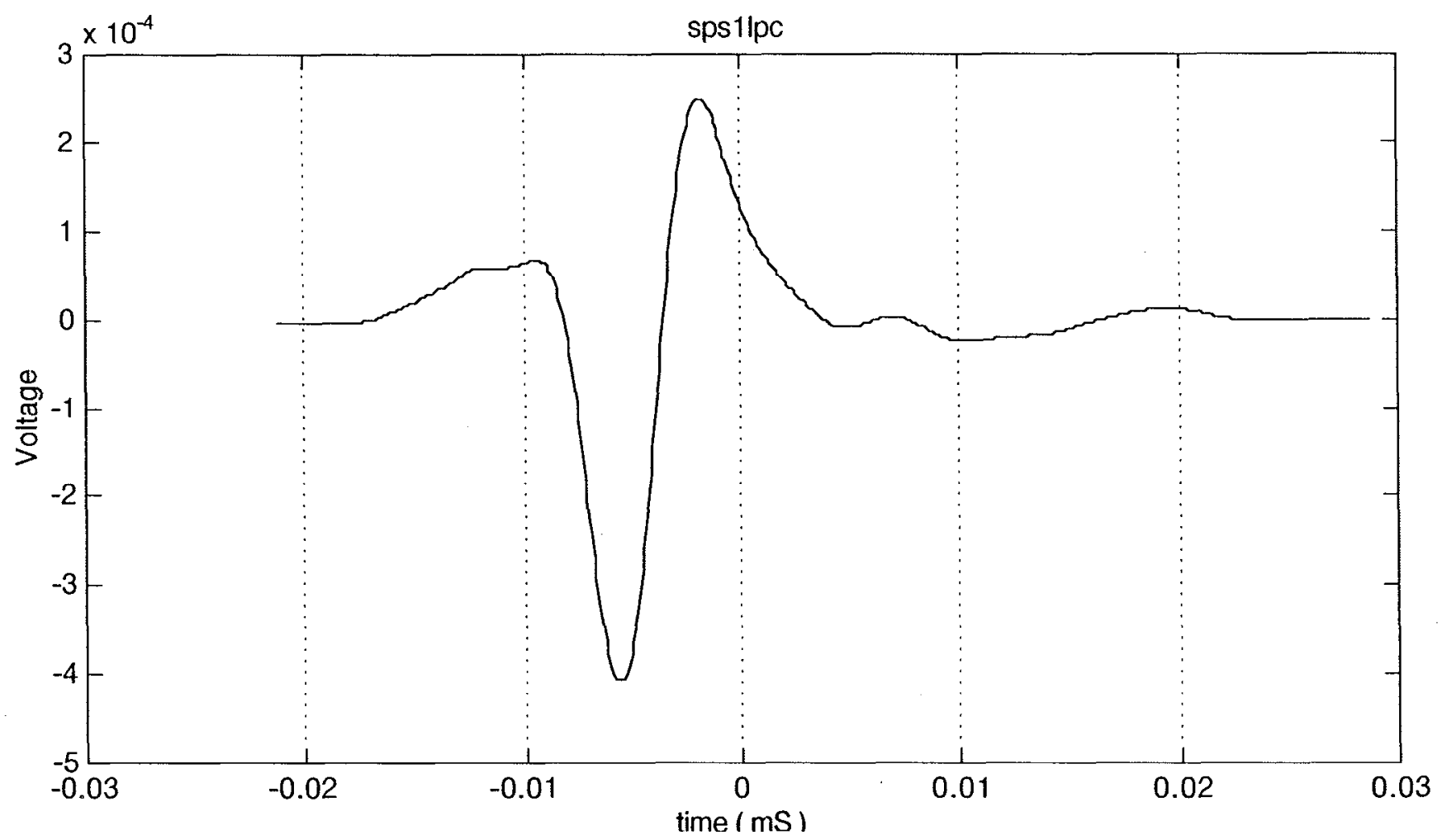

Figure C18. The received signal from an uncompensated Superposed Gaussian equivalent piston pulse at 600 feet separation. 


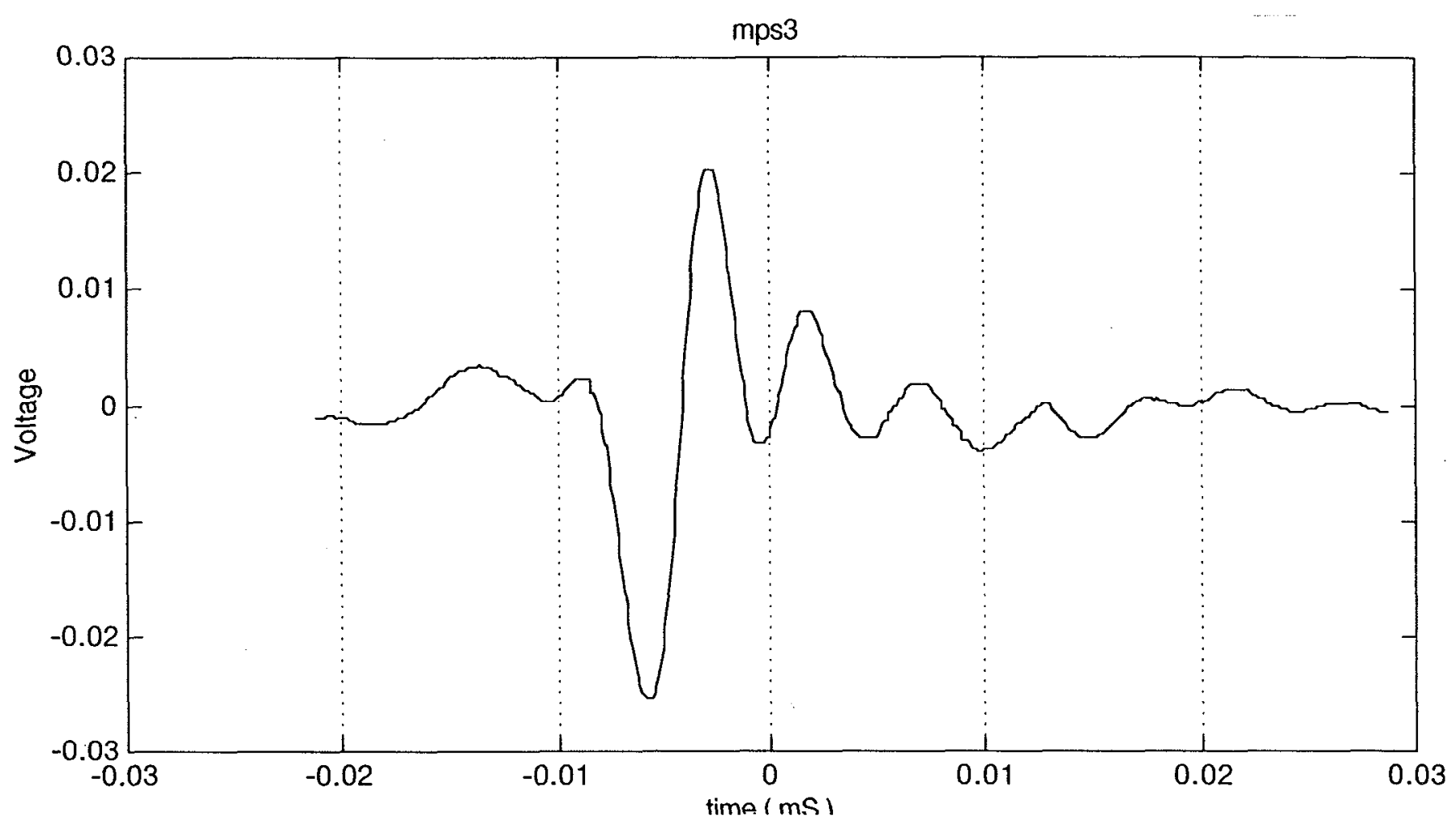

Figure C19. The received signal from an uncompensated Modified Pulse Spectrum 3 pulse at 600 feet separation.

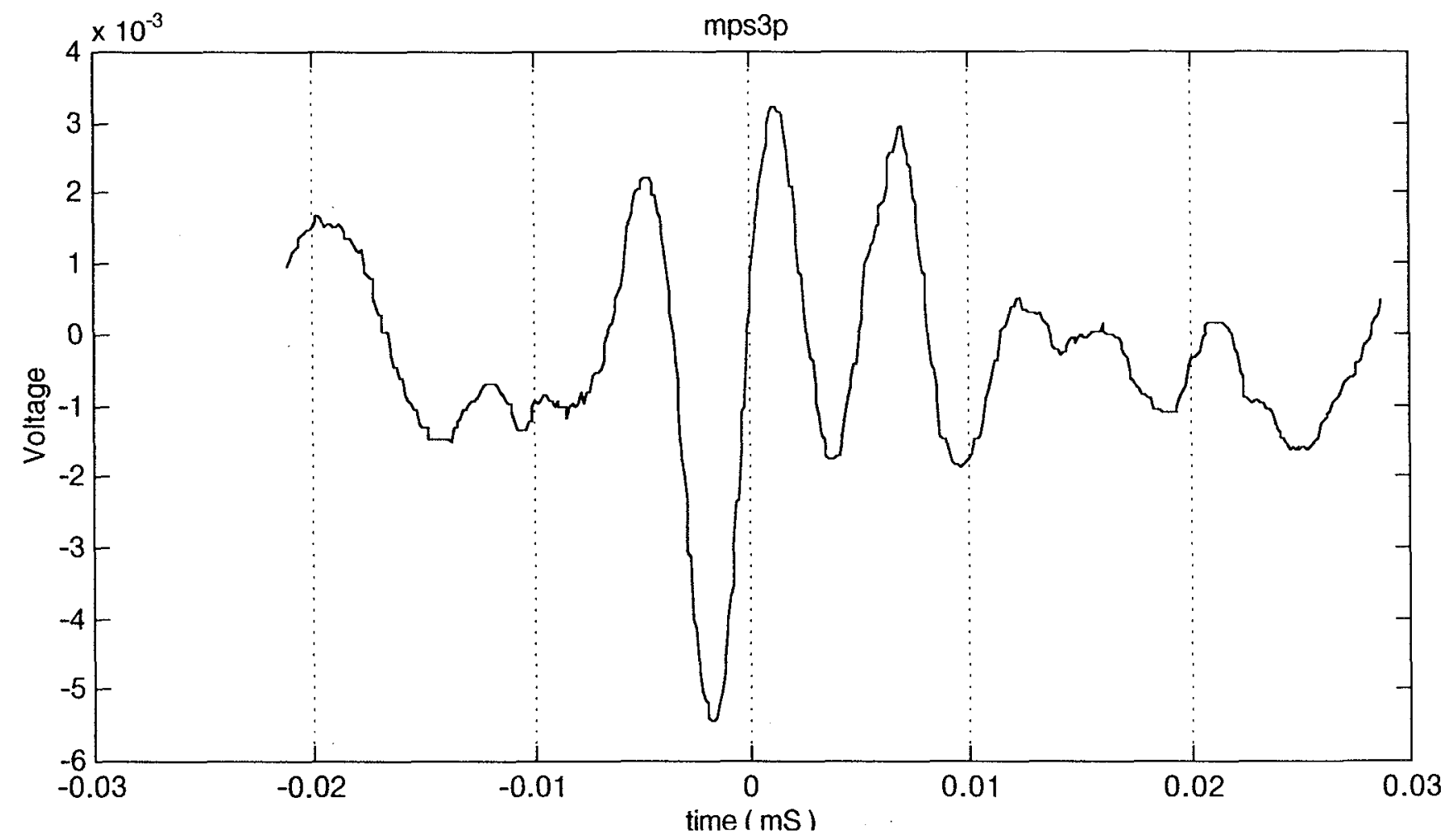

Figure C20. The received signal from an uncompensated Modified Pulse Spectrum 3 equivalent piston pulse at 600 feet separation. 

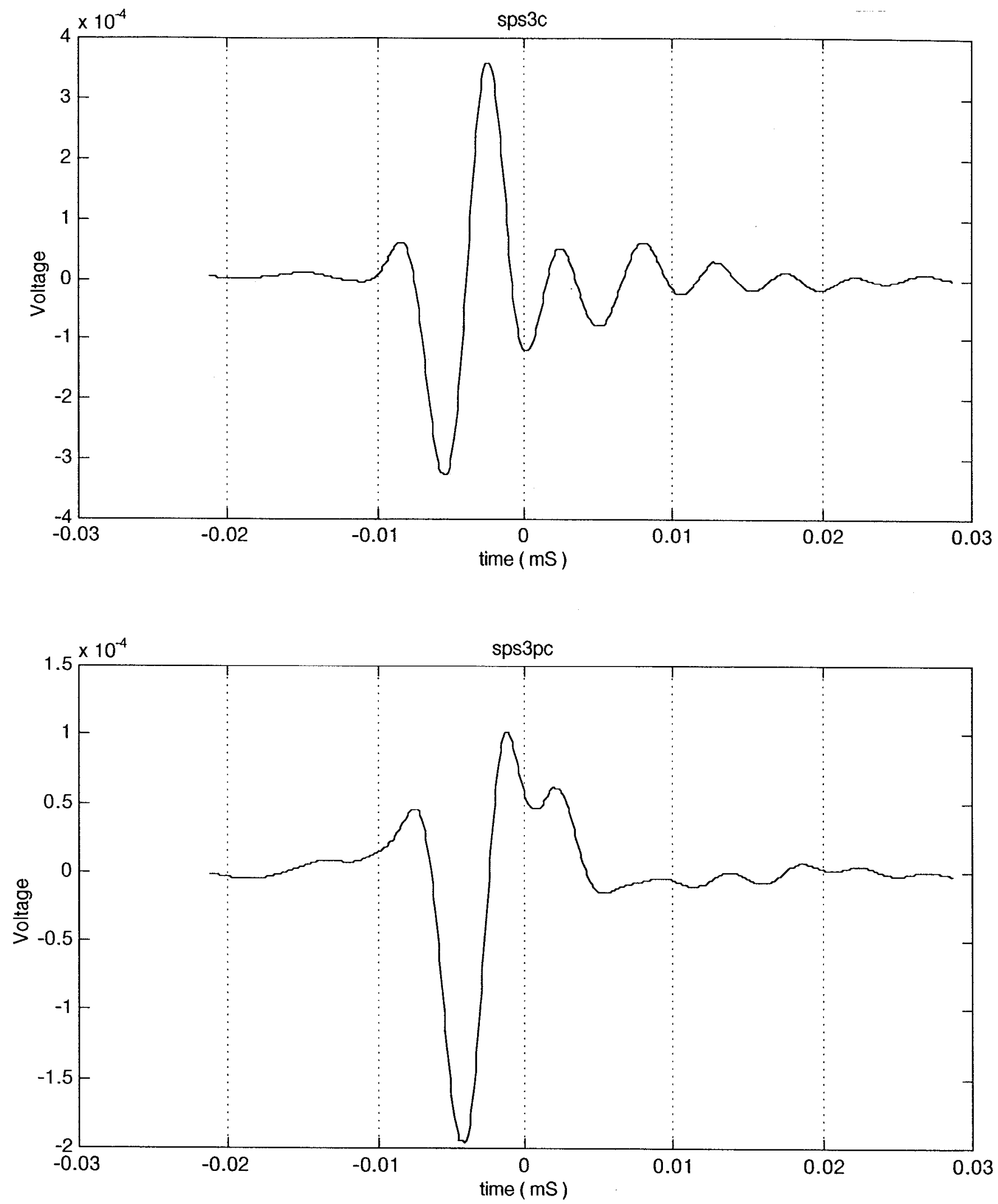

Figure C22. The received signal from a stretched and recompressed Modified Pulse Spectrum pulse equivalent piston at 600 feet separation. 


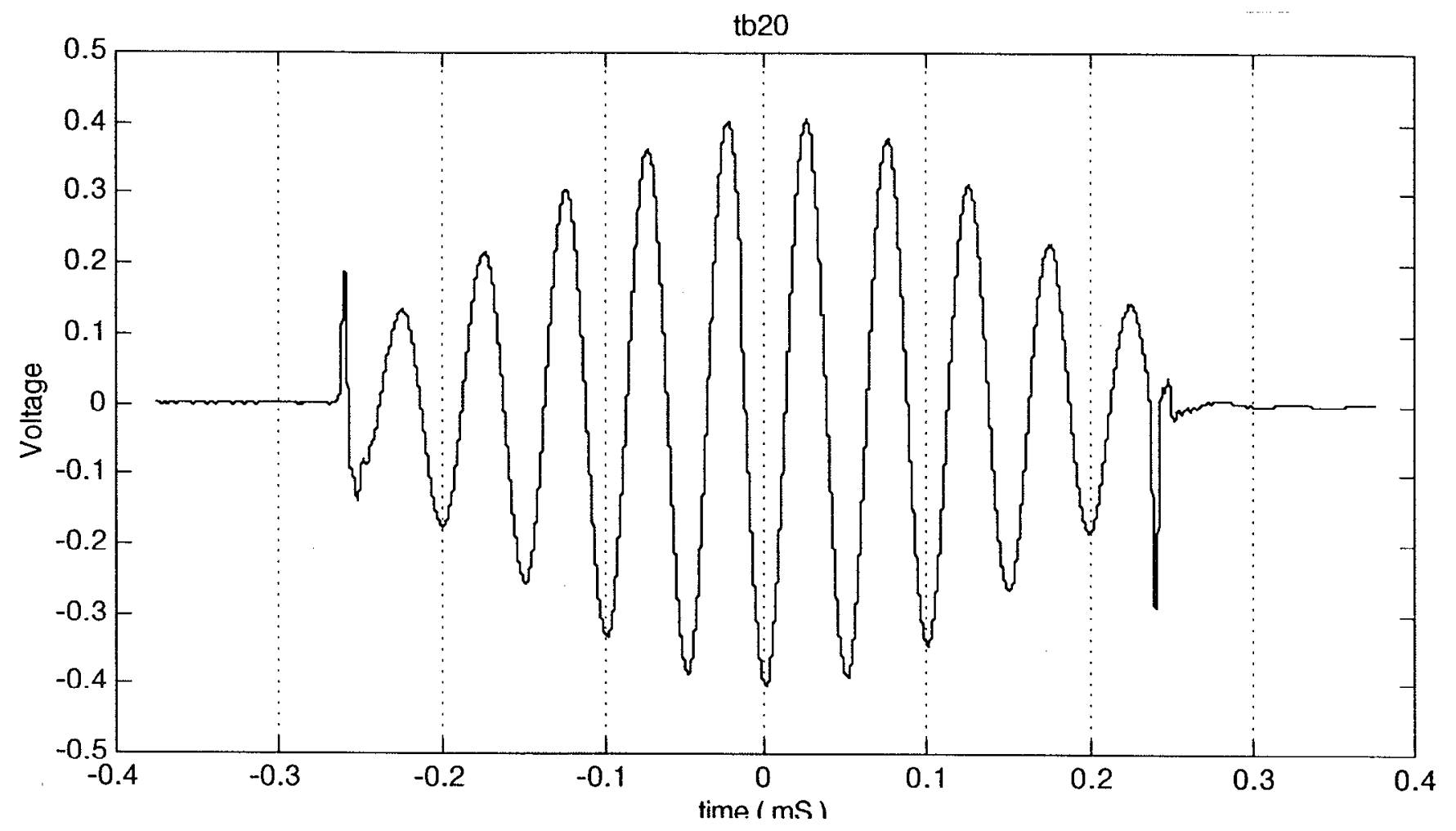

Figure $\mathrm{C} 23$. The received signal from a $20 \mathrm{kHz}$ tone burst at 100 feet separation.

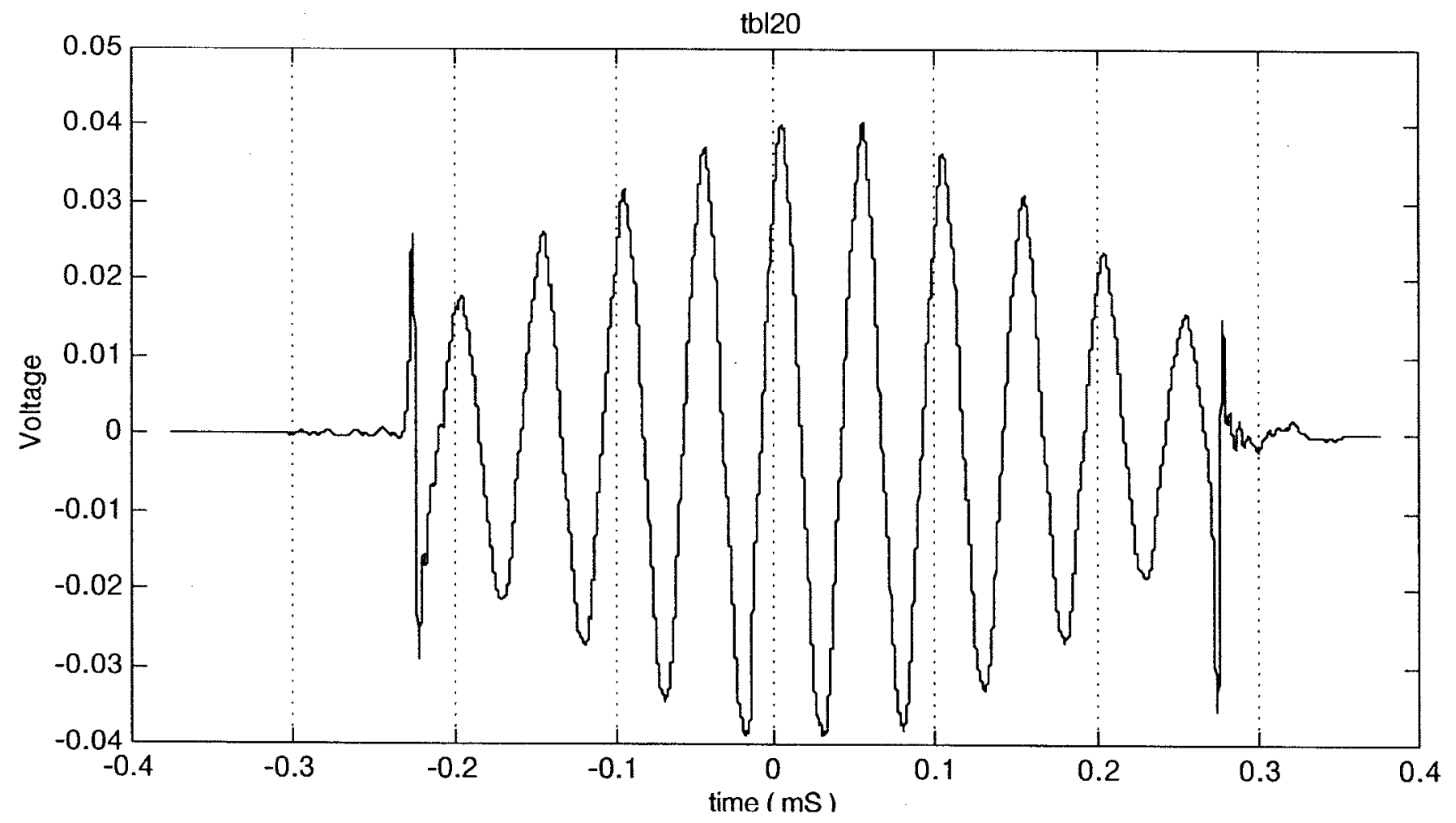

Figure $\mathrm{C} 24$. The received signal from a $20 \mathrm{kHz}$ tone burst at 600 feet separation. 


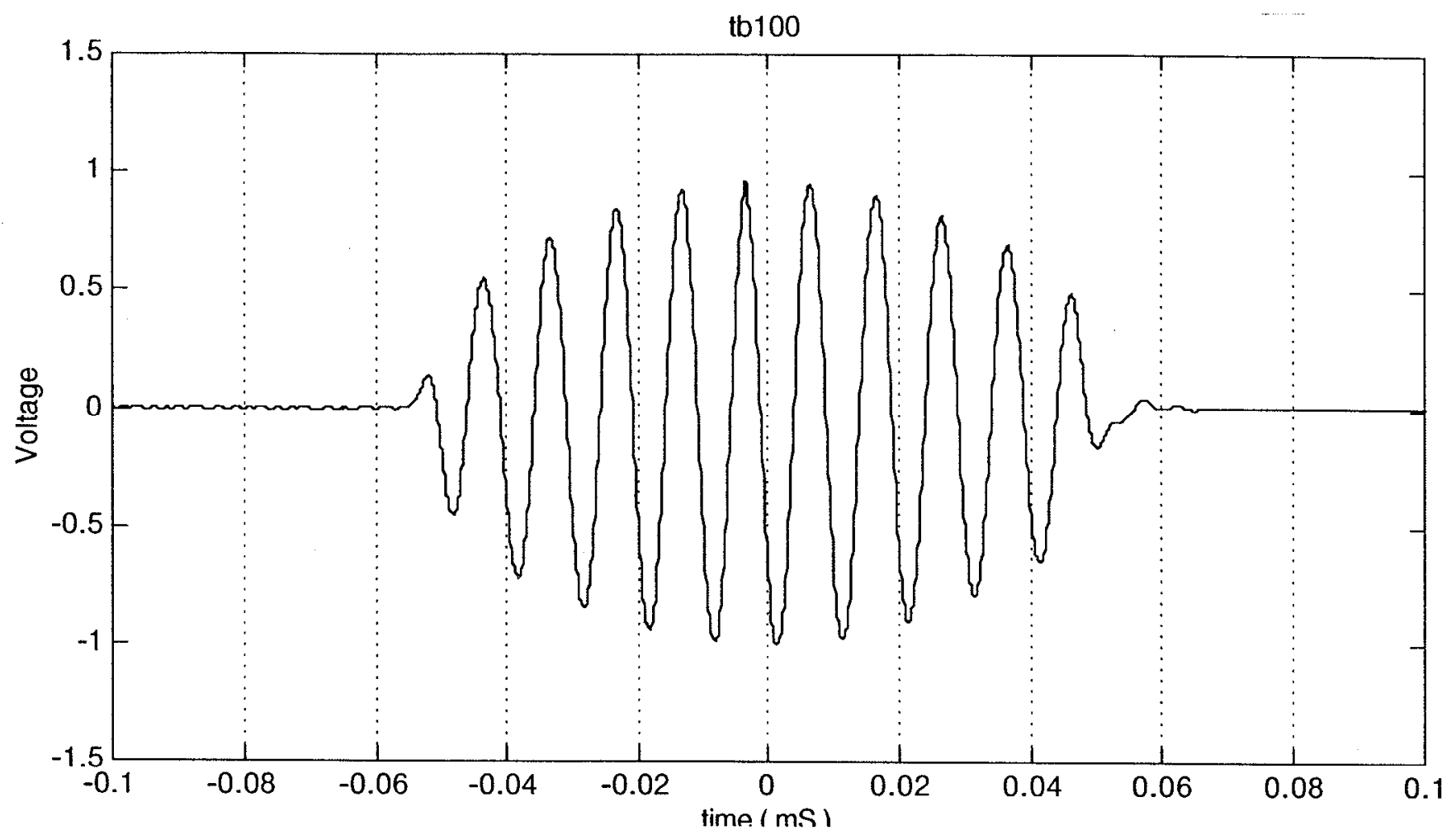

Figure C25. The received signal from a $100 \mathrm{kHz}$ tone burst at 100 feet separation.

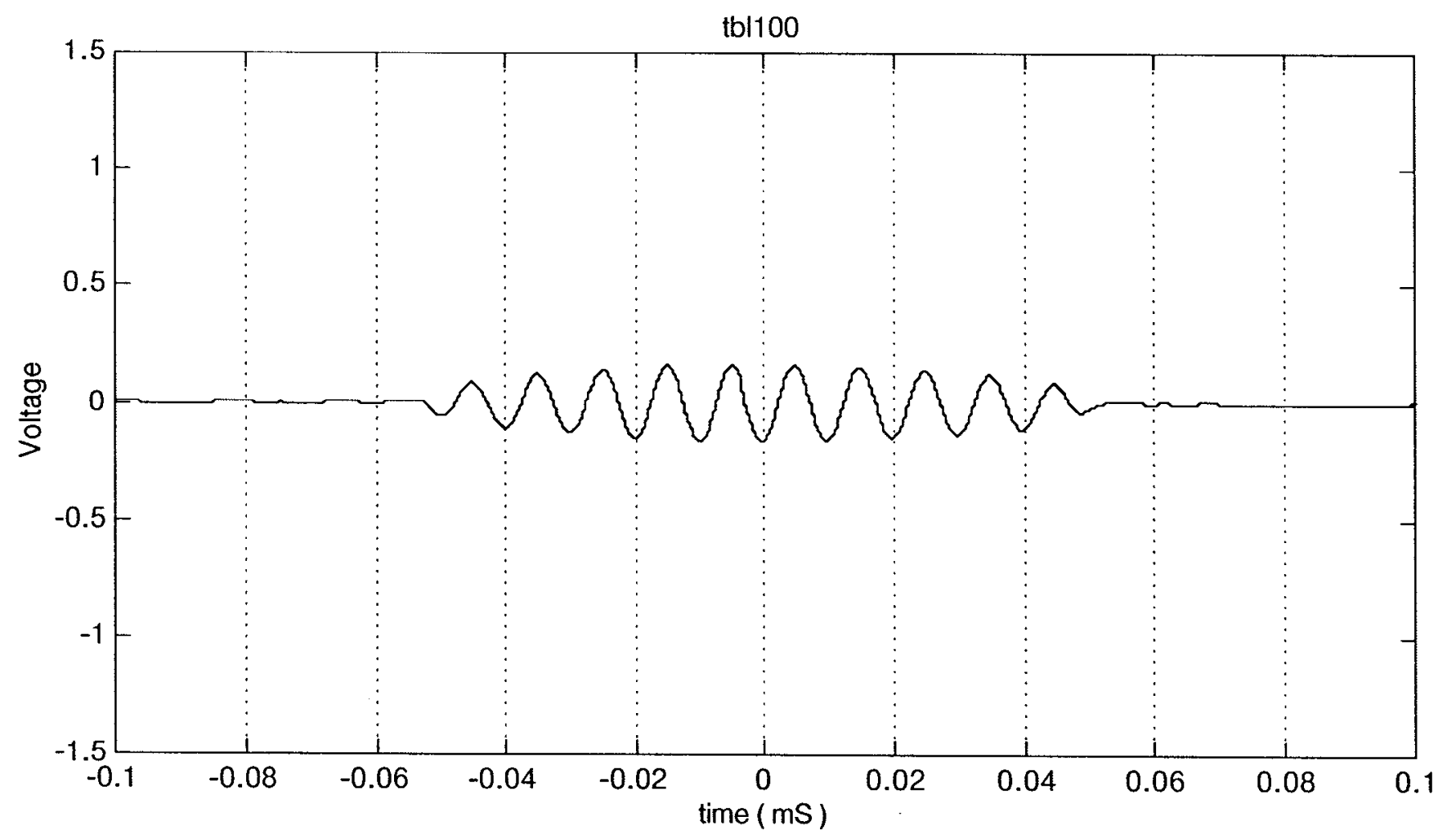

Figure C26. The received signal from a $100 \mathrm{kHz}$ tone burst at 600 feet separation. 


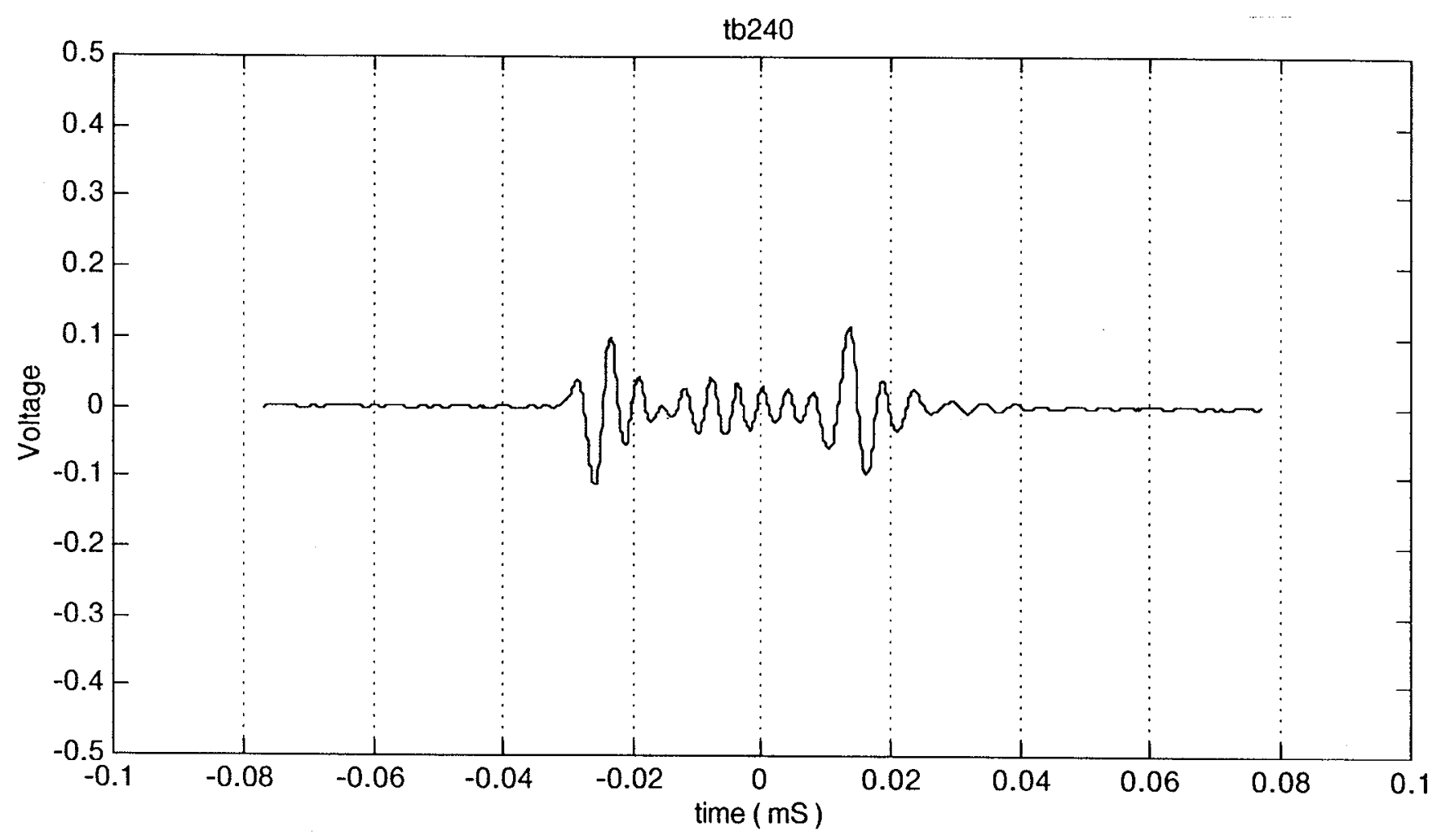

Figure C27. The received signal from a $240 \mathrm{kHz}$ tone burst at 100 feet separation.

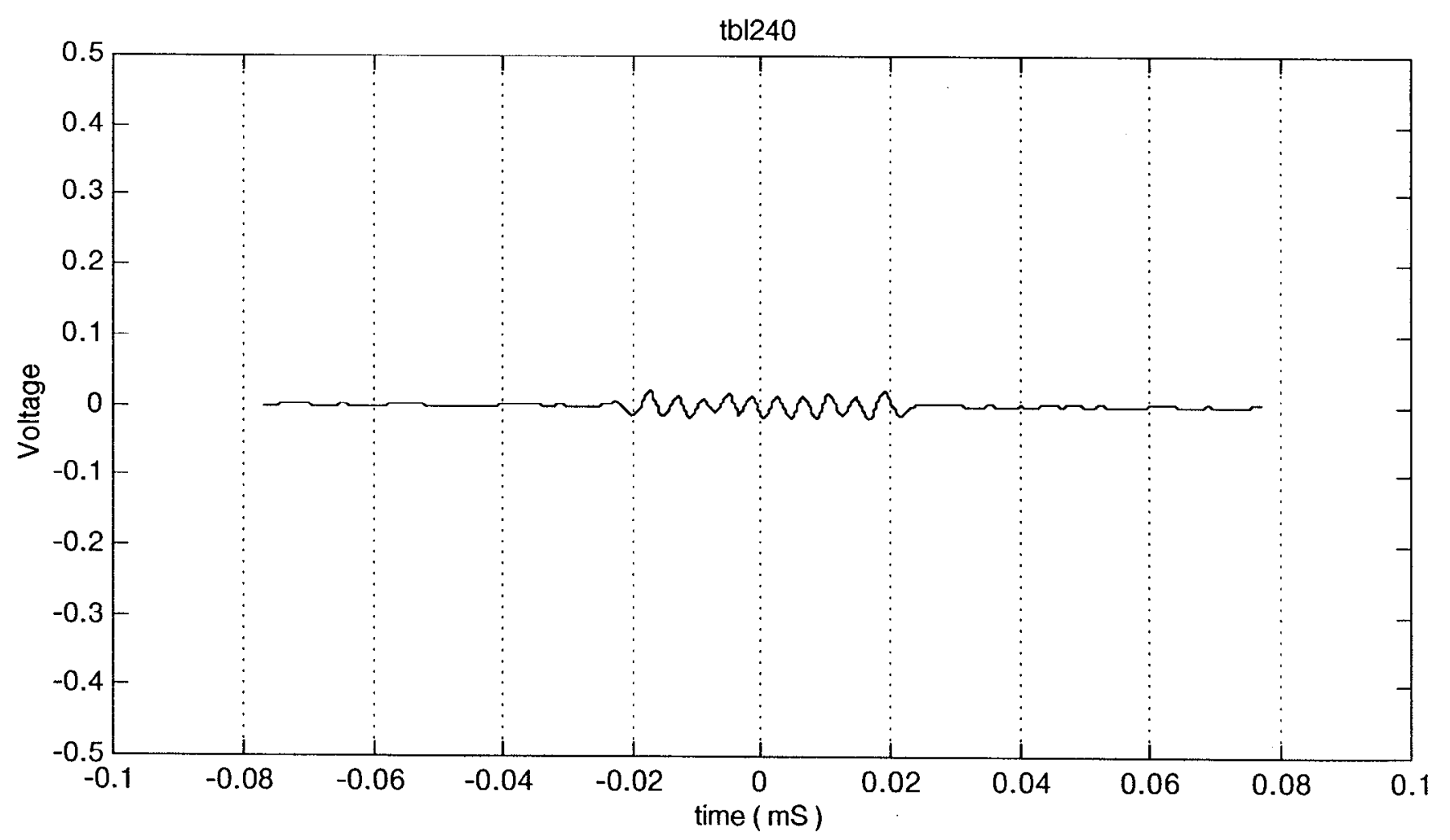

Figure C28. The received signal from a $240 \mathrm{kHz}$ tone burst at 600 feet separation. 


\section{Appendix D. Angular Spectra Data.}

This section presents the data measurements as the energy detected for different source functions as $\mathrm{dB}$ for angle and frequency. The data is presented as a color map on a scale from 0 to $-60 \mathrm{~dB}$ relative power. Frequency is the horizontal axis, and angle is the vertical axis.

For those sets for which we ran both a Localized Wave case and an equivalent piston pulse case, the spectra are displayed together. MPS1NC is an uncompensated MPS1 pulse, MPS12d is a $2^{\text {nd }}$ derivative compensated MPS1 pulse, MPS3 piston compressed is an uncompensated MPS3 pulse stretched and recompressed.

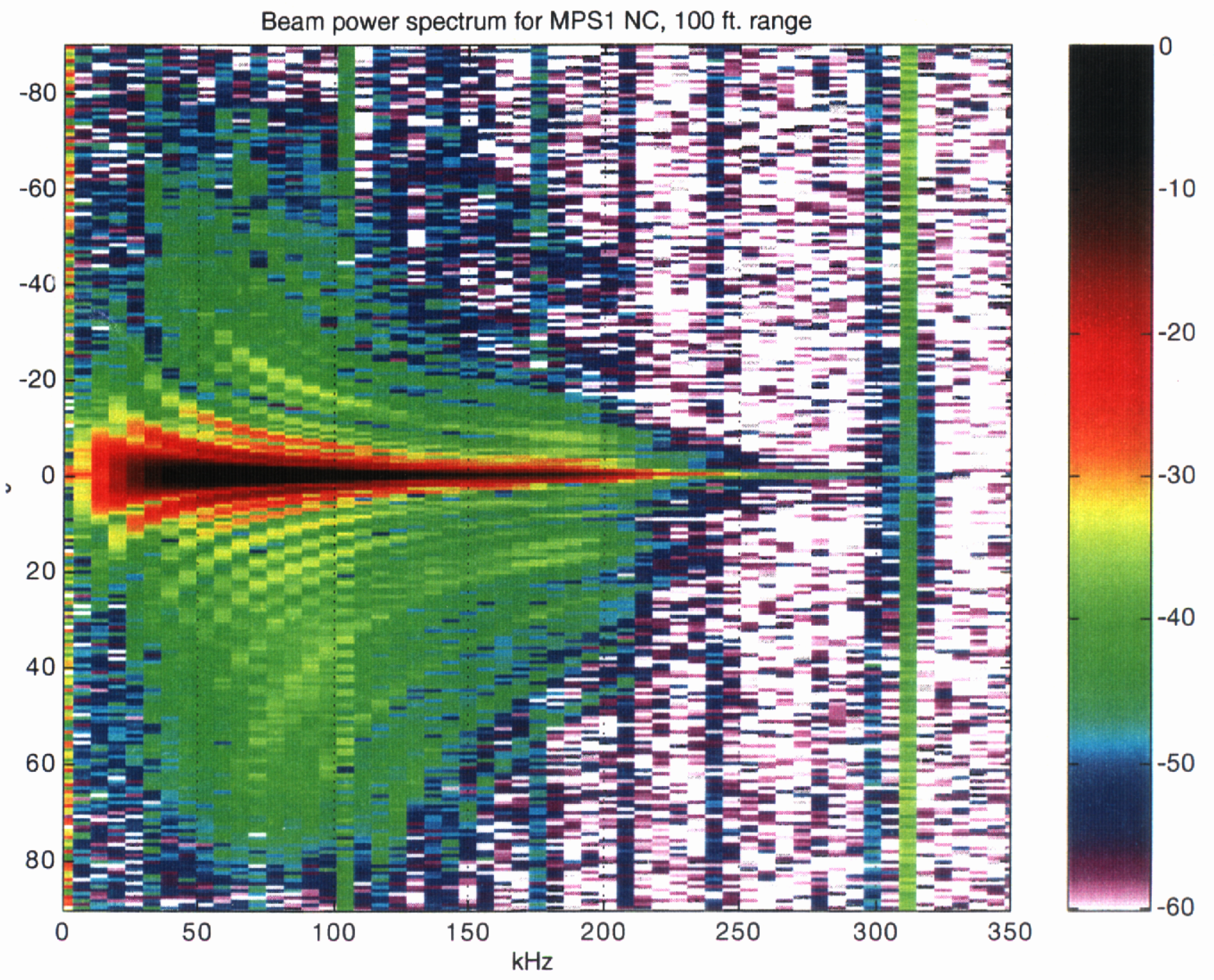




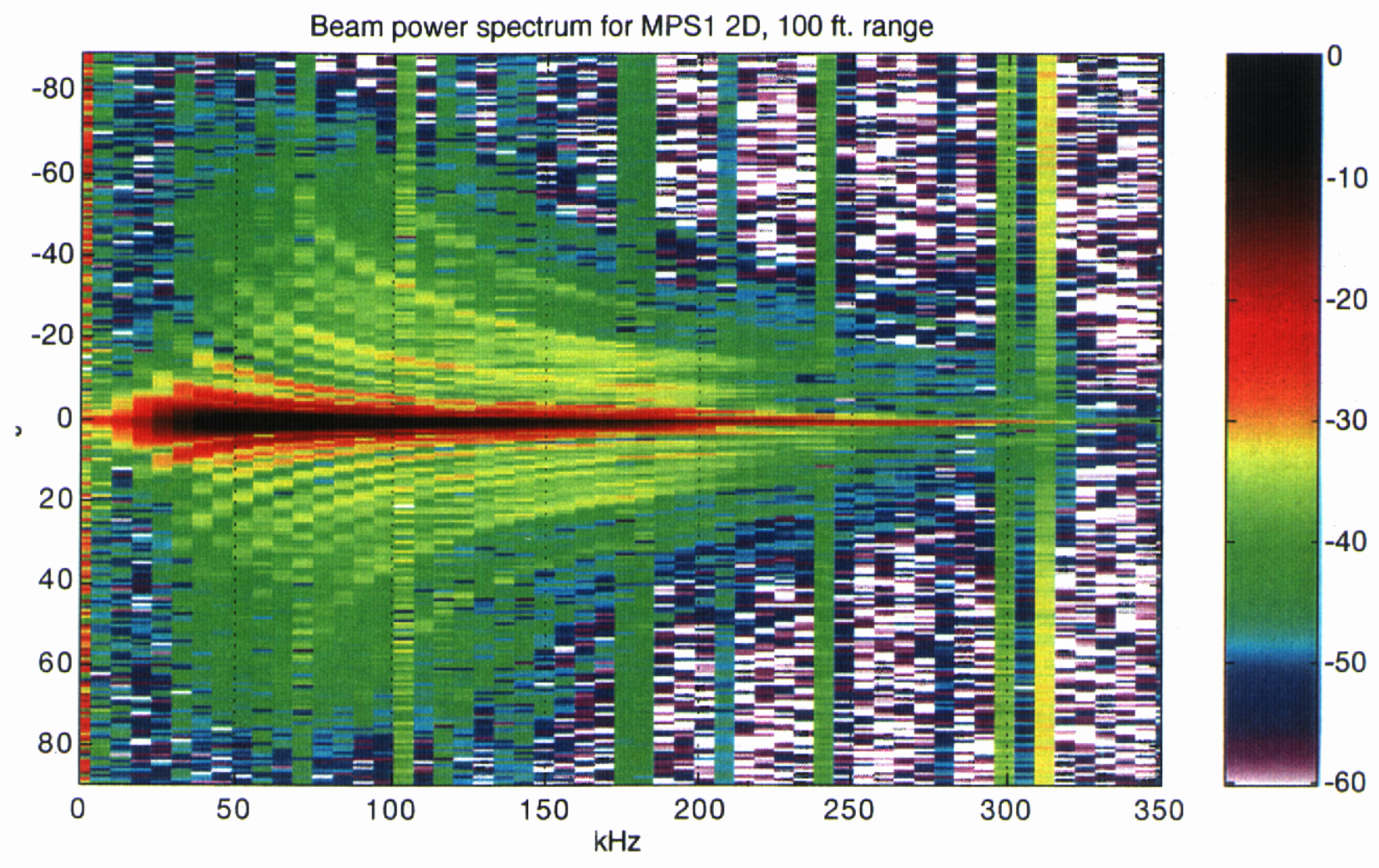

Beam power spectrum for MPS12D piston, $100 \mathrm{ft}$. range

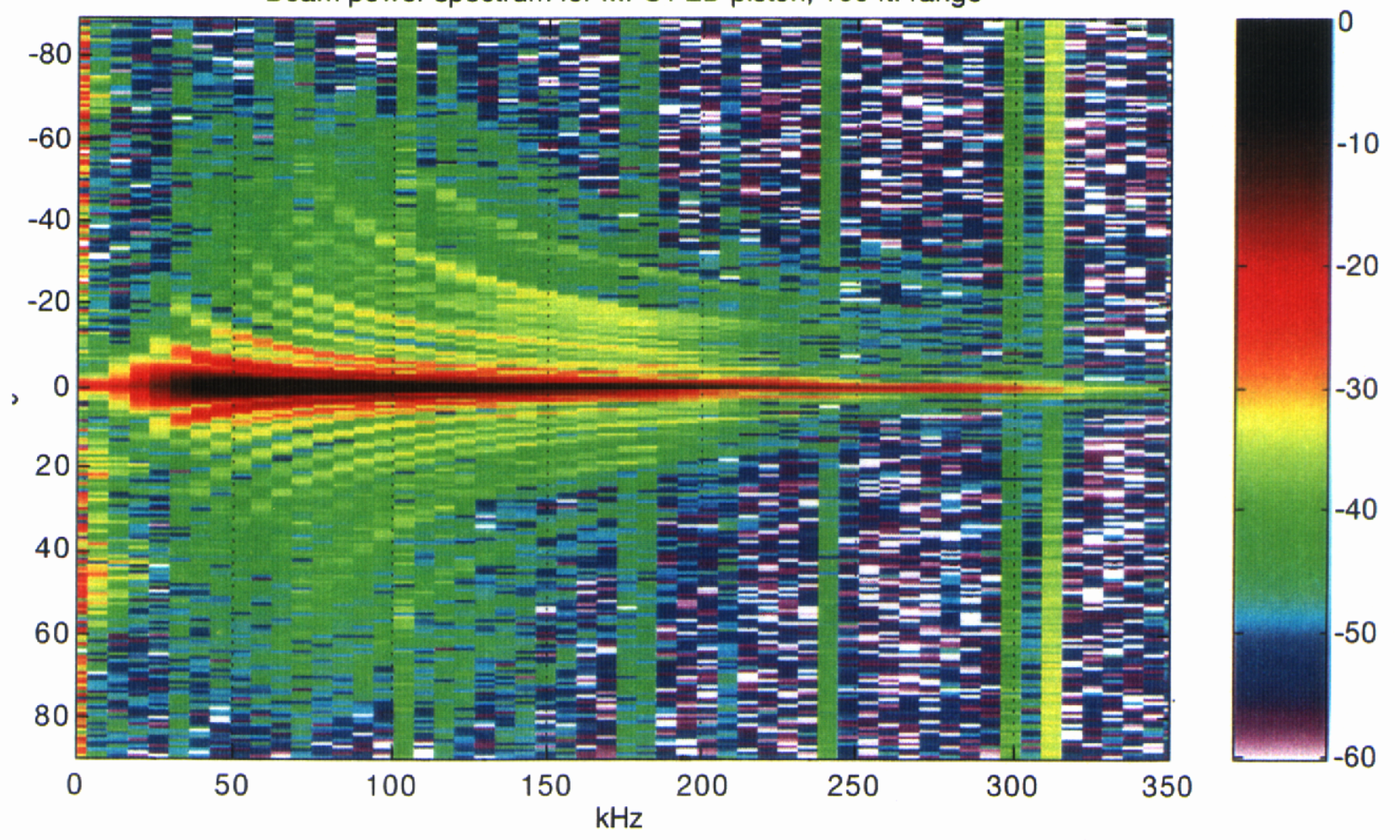

Appendix D 
Beam power spectrum for MPS12D, $200 \mathrm{yd}$. range

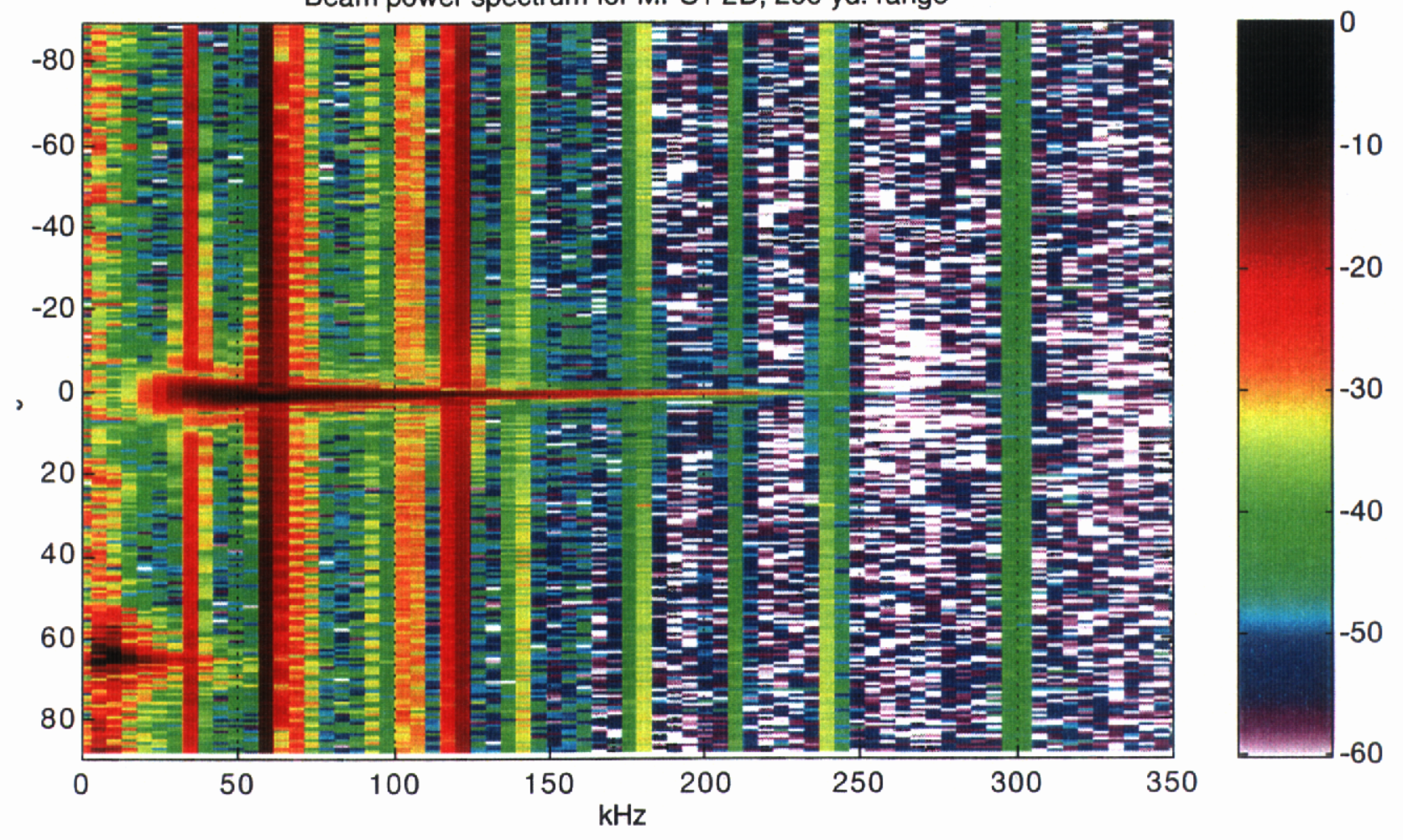

Beam power spectrum for MPS12D piston, $200 \mathrm{yd}$. range
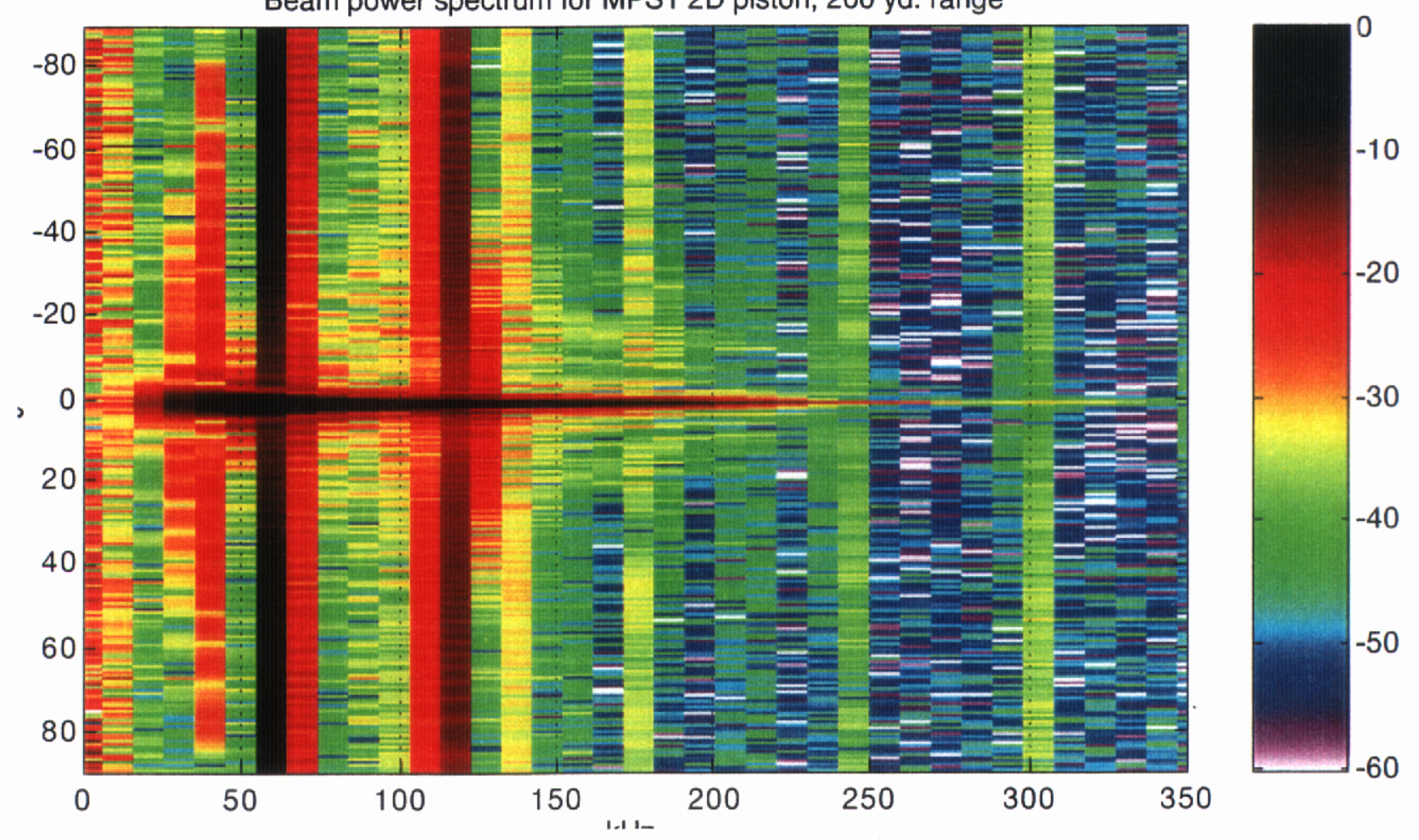
Beam power spectrum for MPS1 3D, $100 \mathrm{ft}$. range
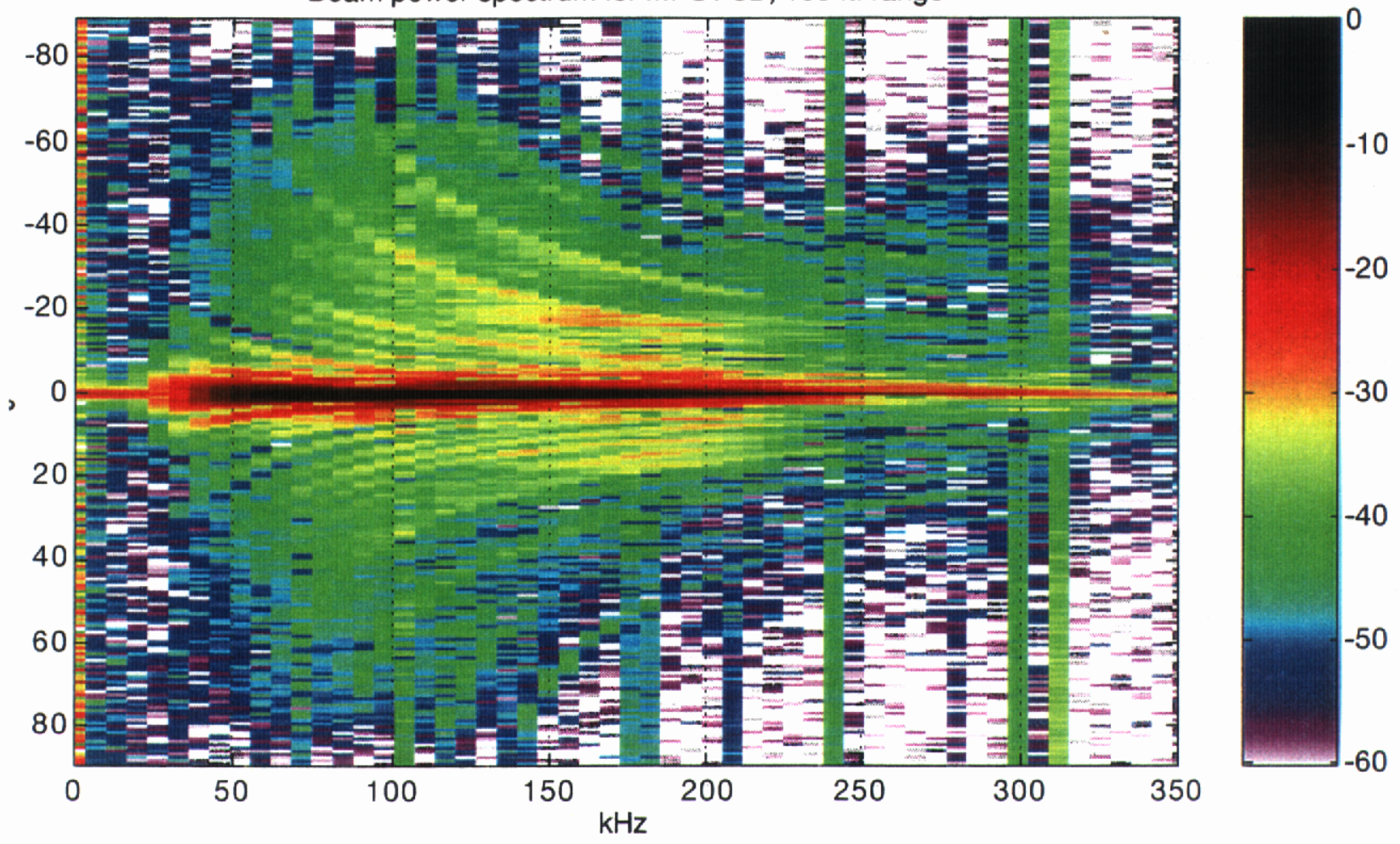

Beam power spectrum for MPS1 3D piston, $100 \mathrm{ft}$. range
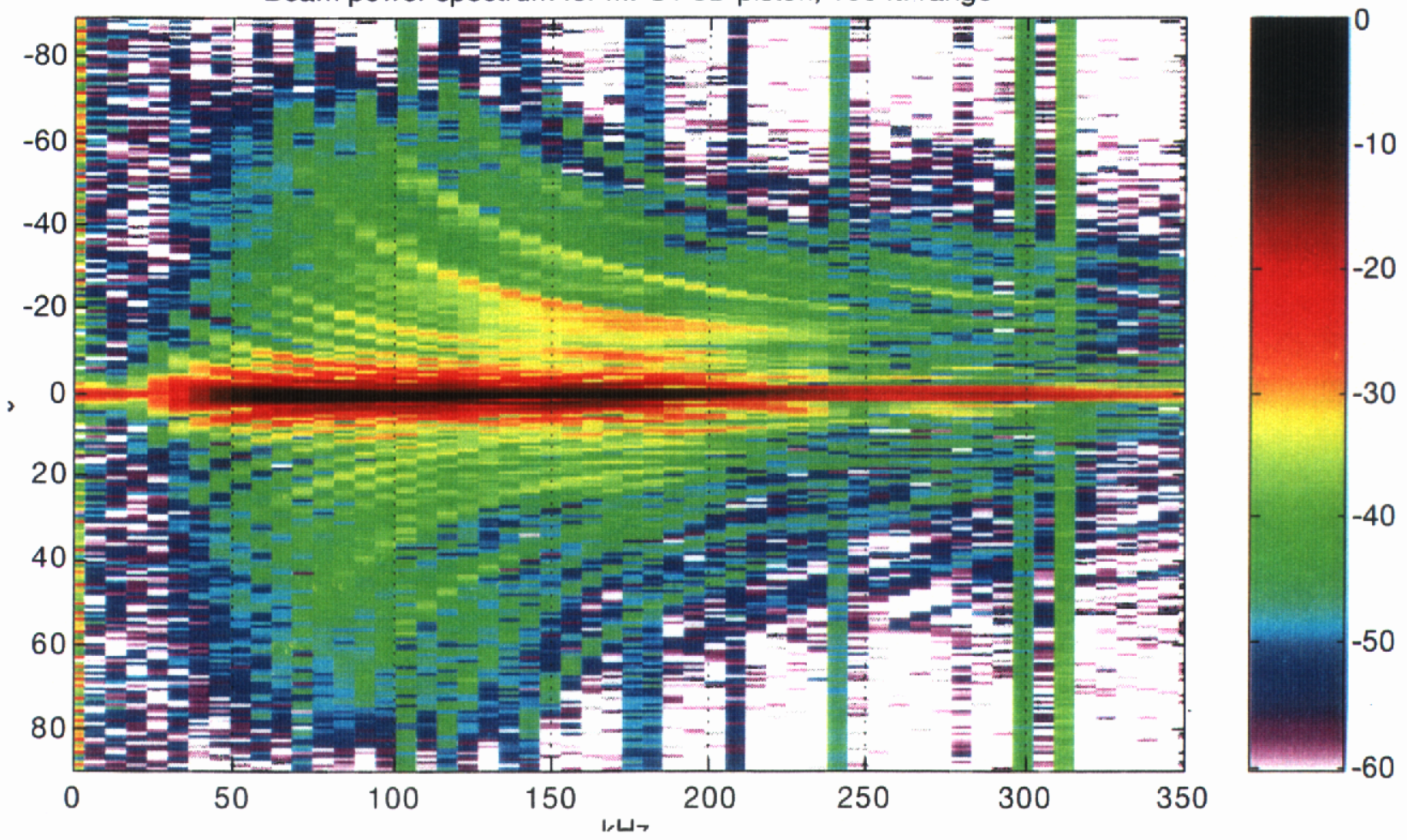

Appendix D 

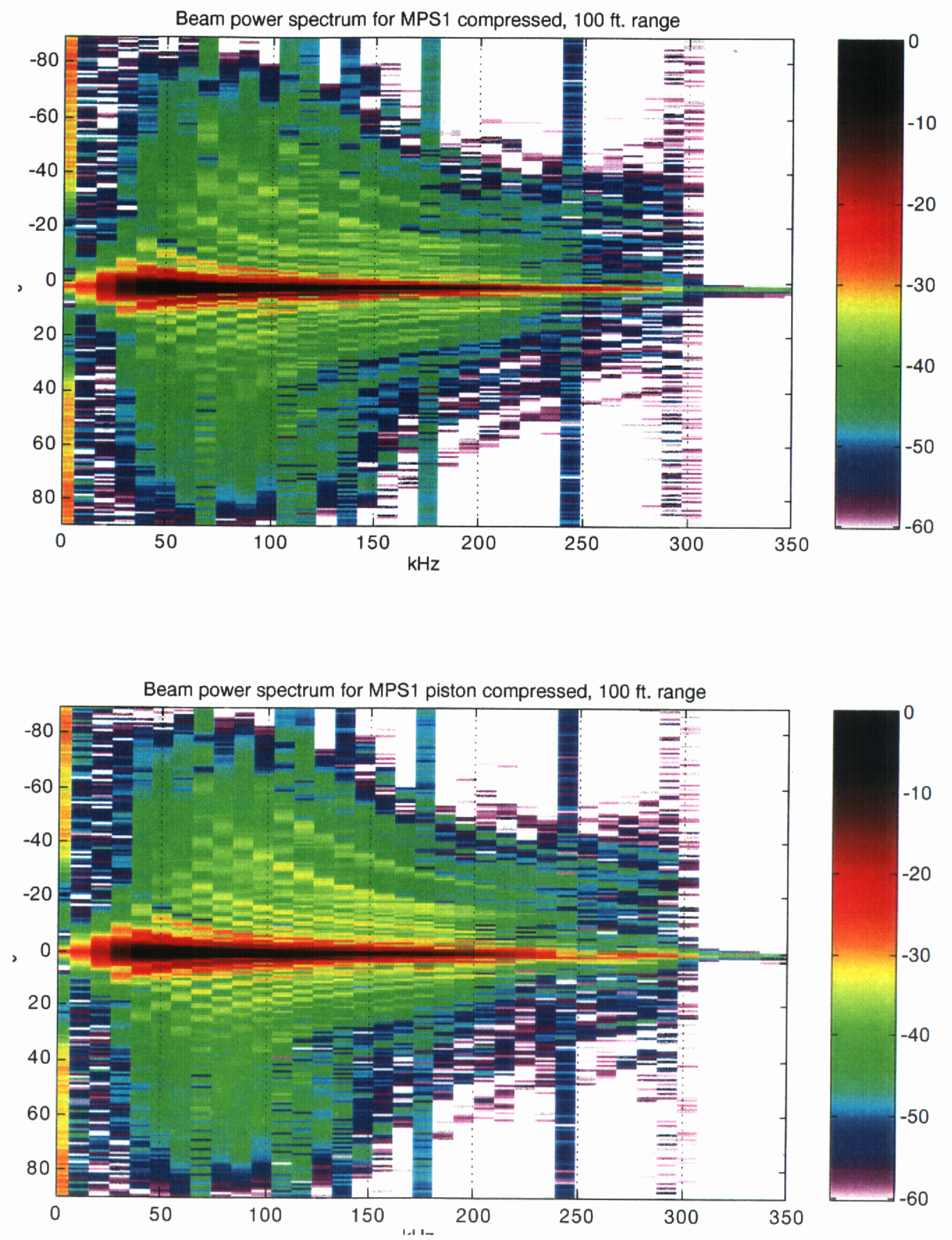
Beam power spectrum for MPS1 compressed, 200 yd. range
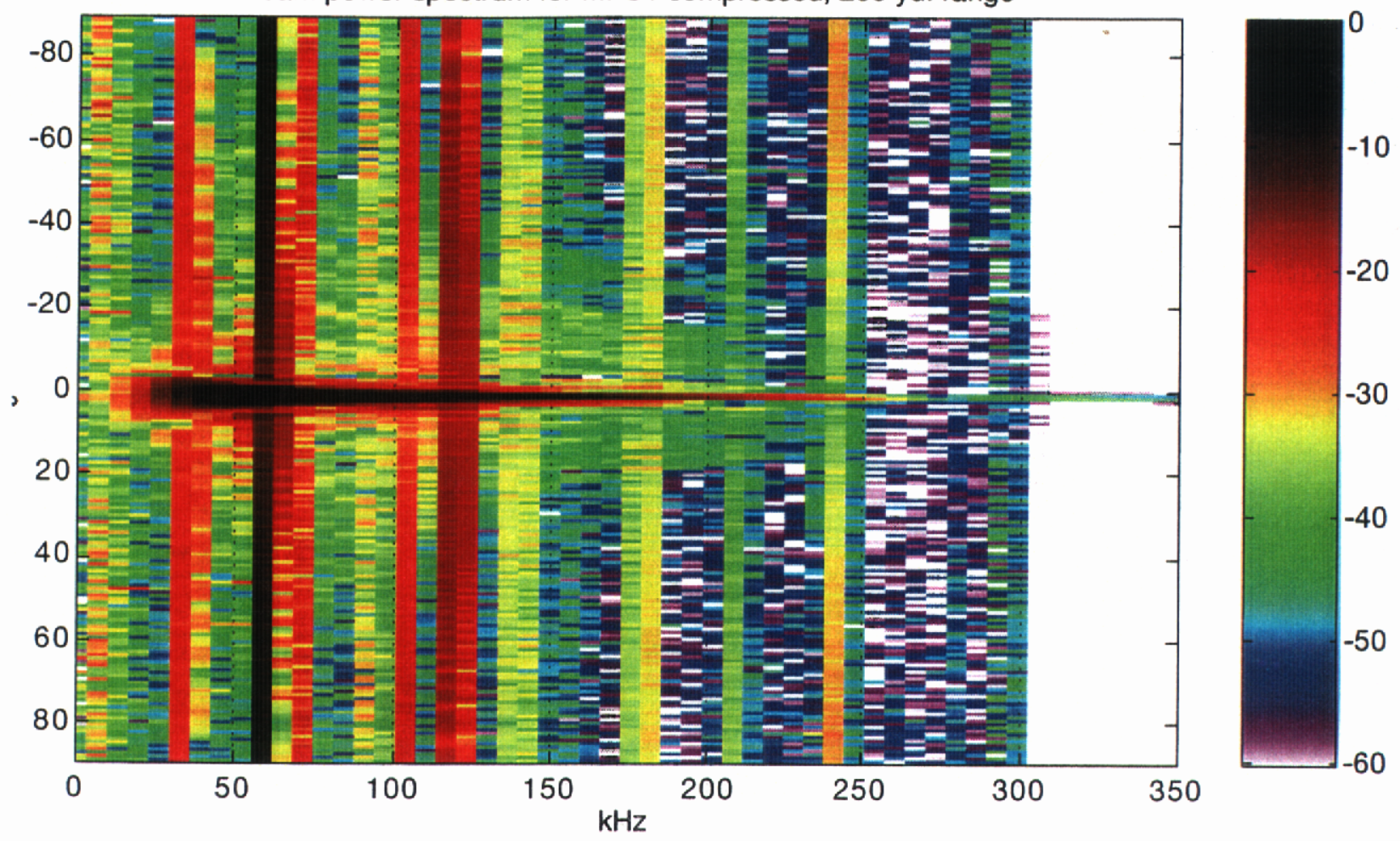

Beam power spectrum for MPS1 piston compressed, 200 yd. range
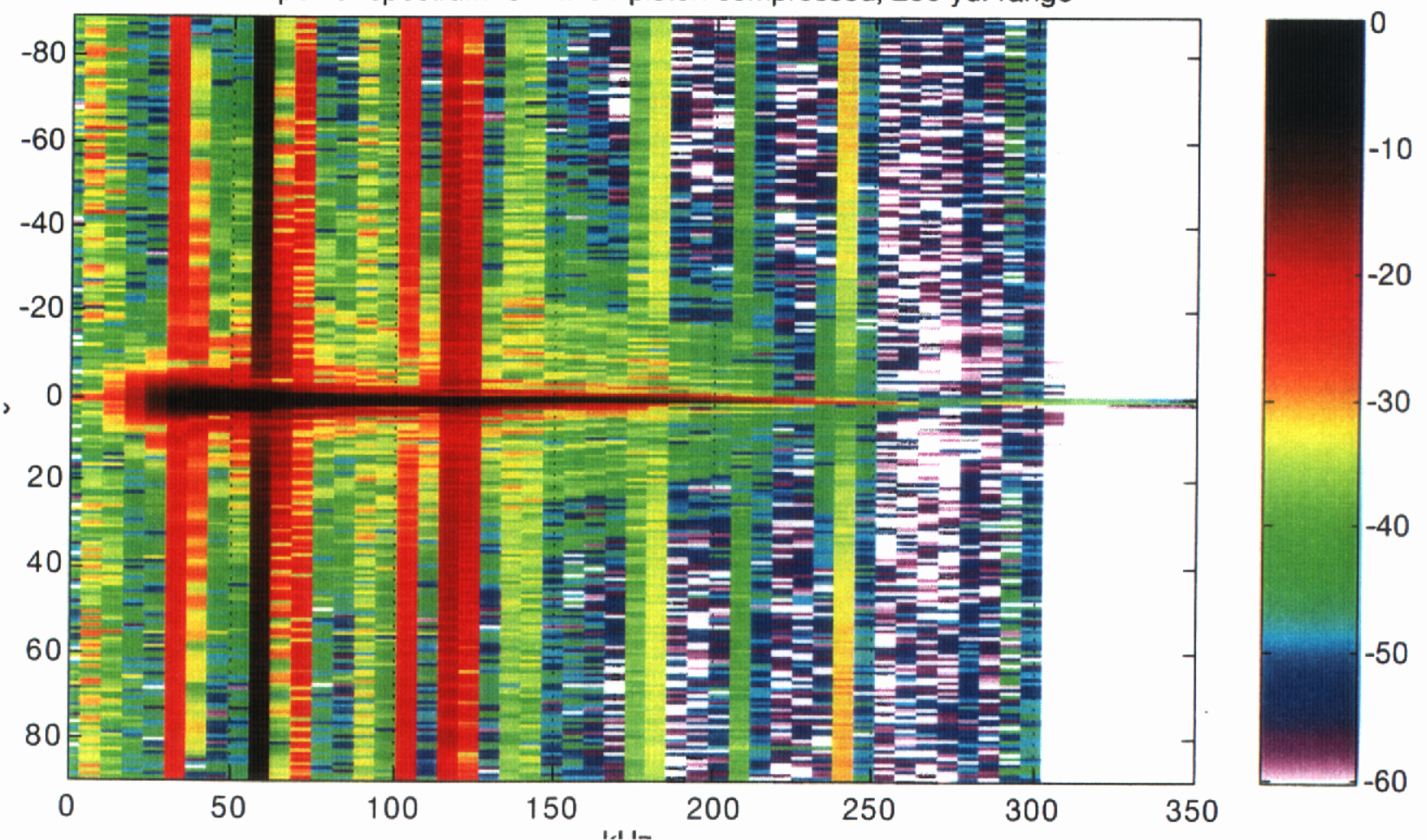
Beam power spectrum for SG1 NC, $100 \mathrm{ft}$. range

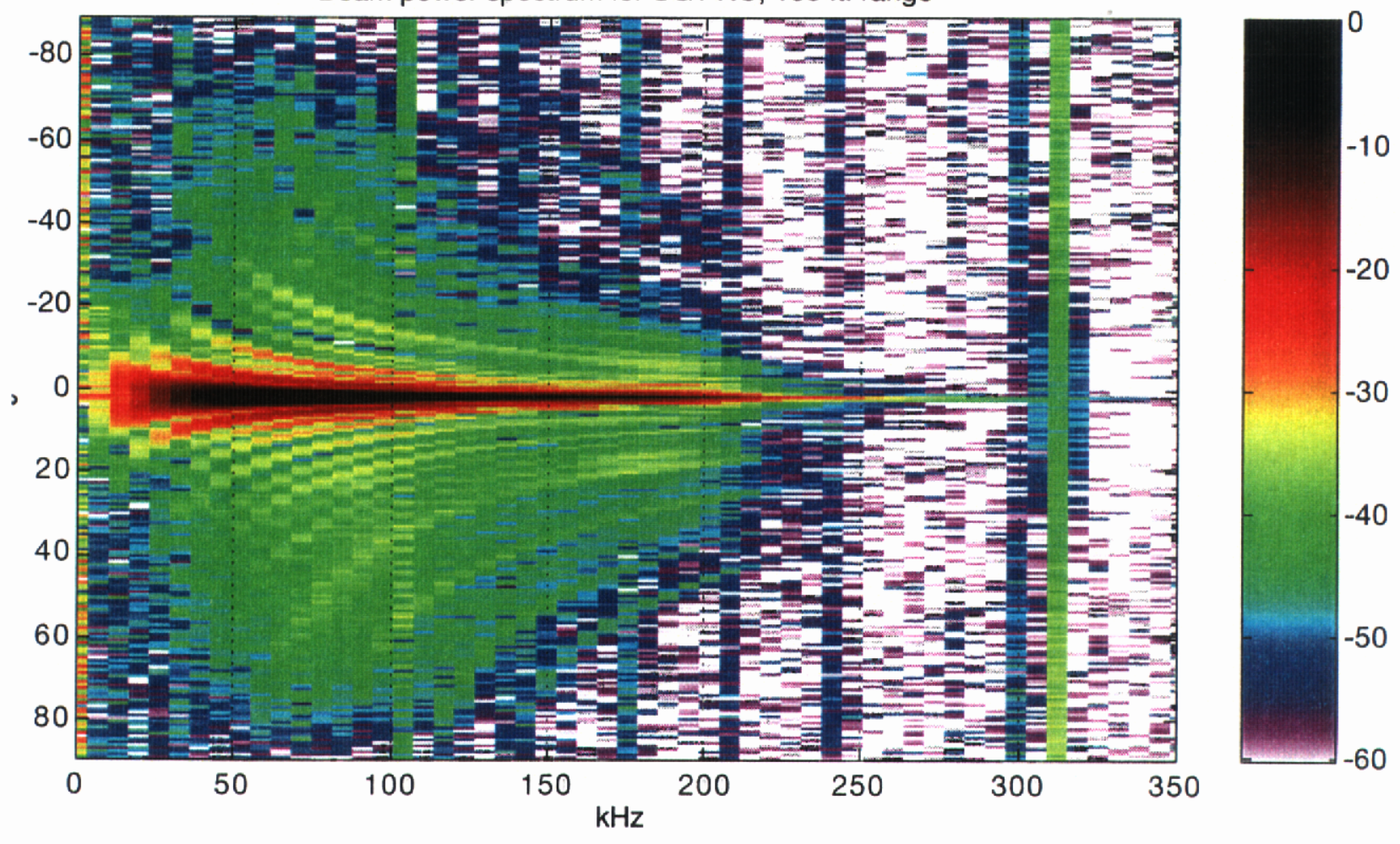

Beam power spectrum for SG1 NC, 200 yd. range

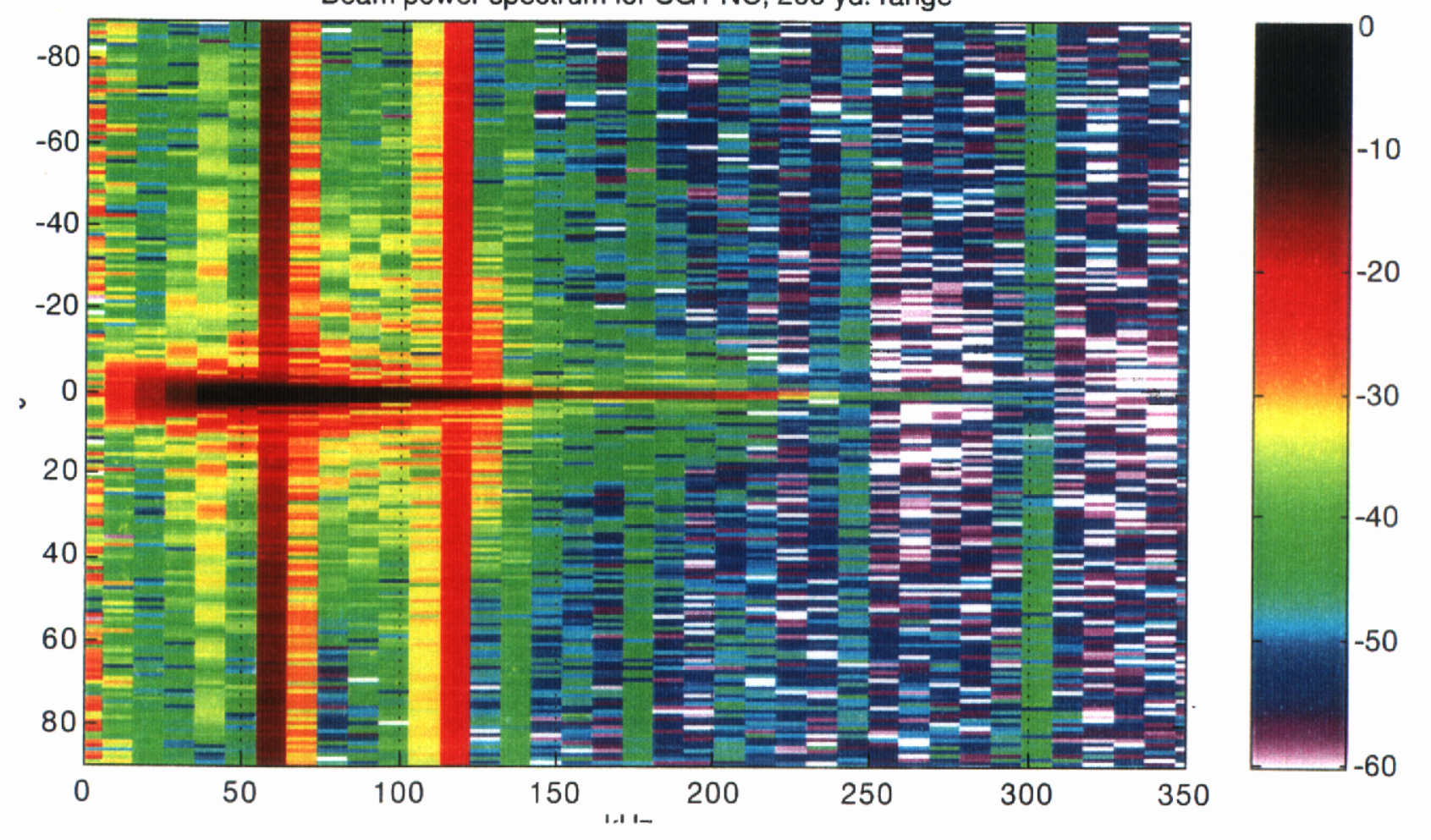


Beam power spectrum for SG1 2D, $100 \mathrm{ft}$. range

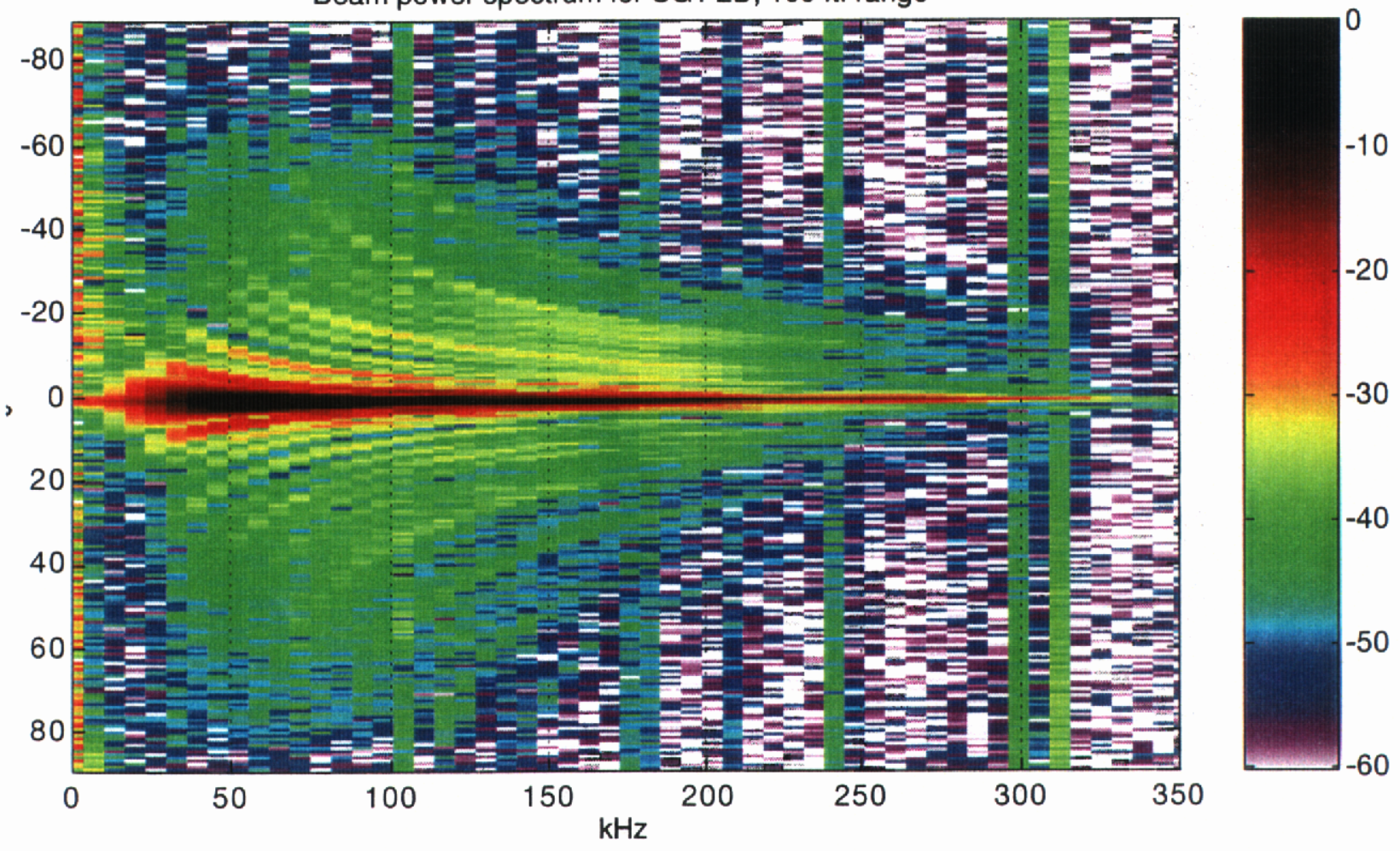

Beam power spectrum for SG1 2D piston, $100 \mathrm{ft}$. range

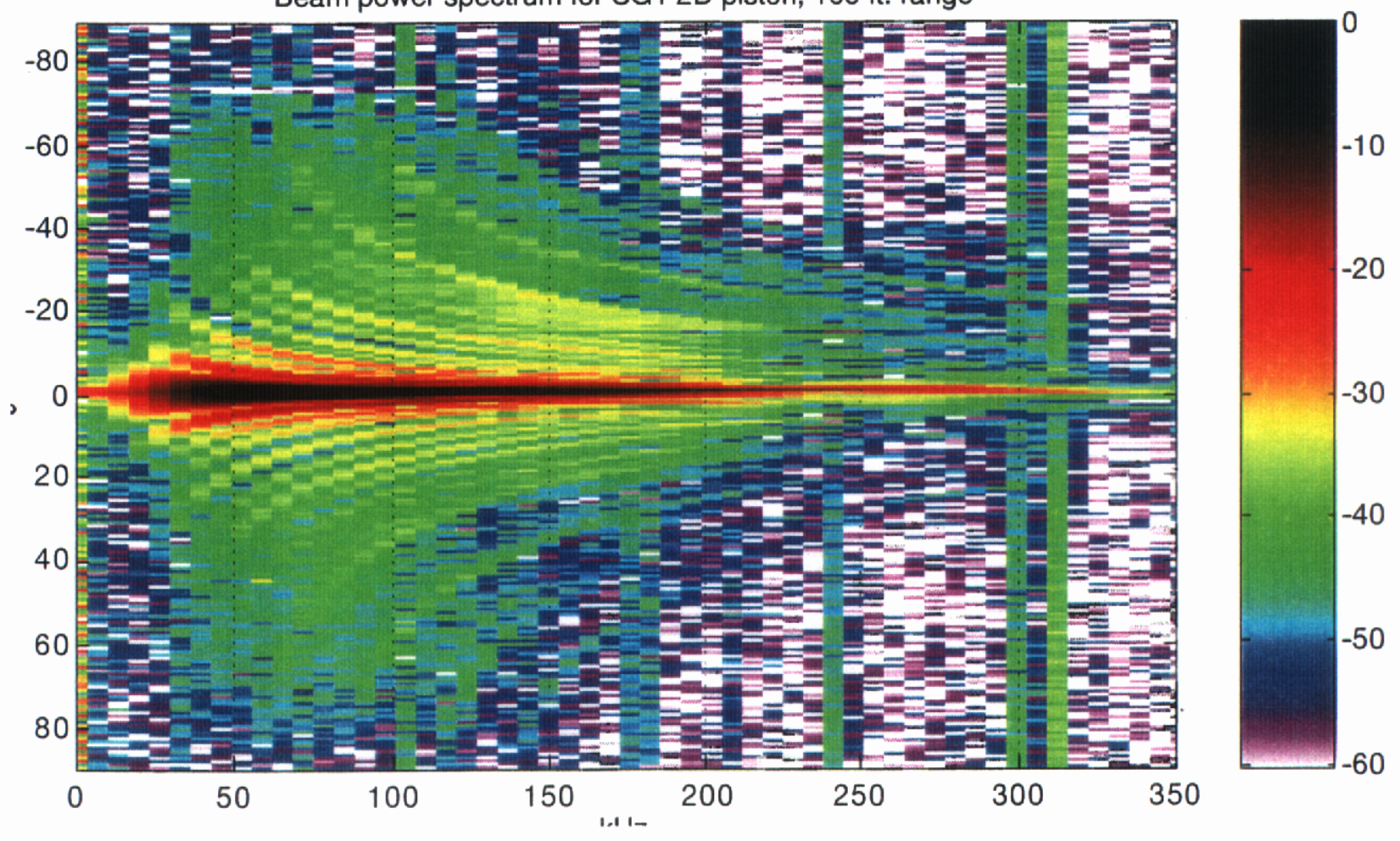


Beam power spectrum for SG1 3D, $100 \mathrm{ft}$. range

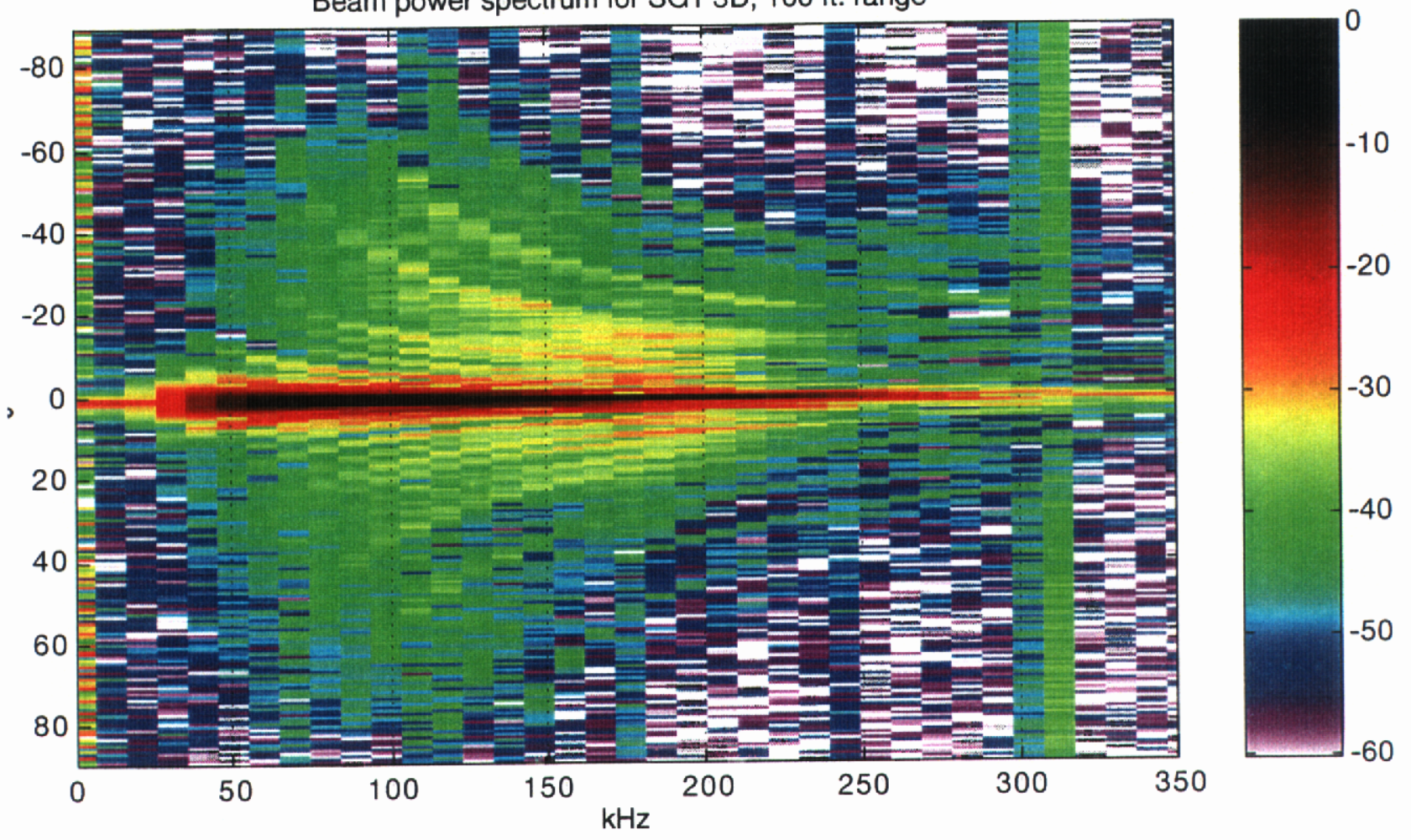

Beam power spectrum for SG1 3D piston, $100 \mathrm{ft}$. range

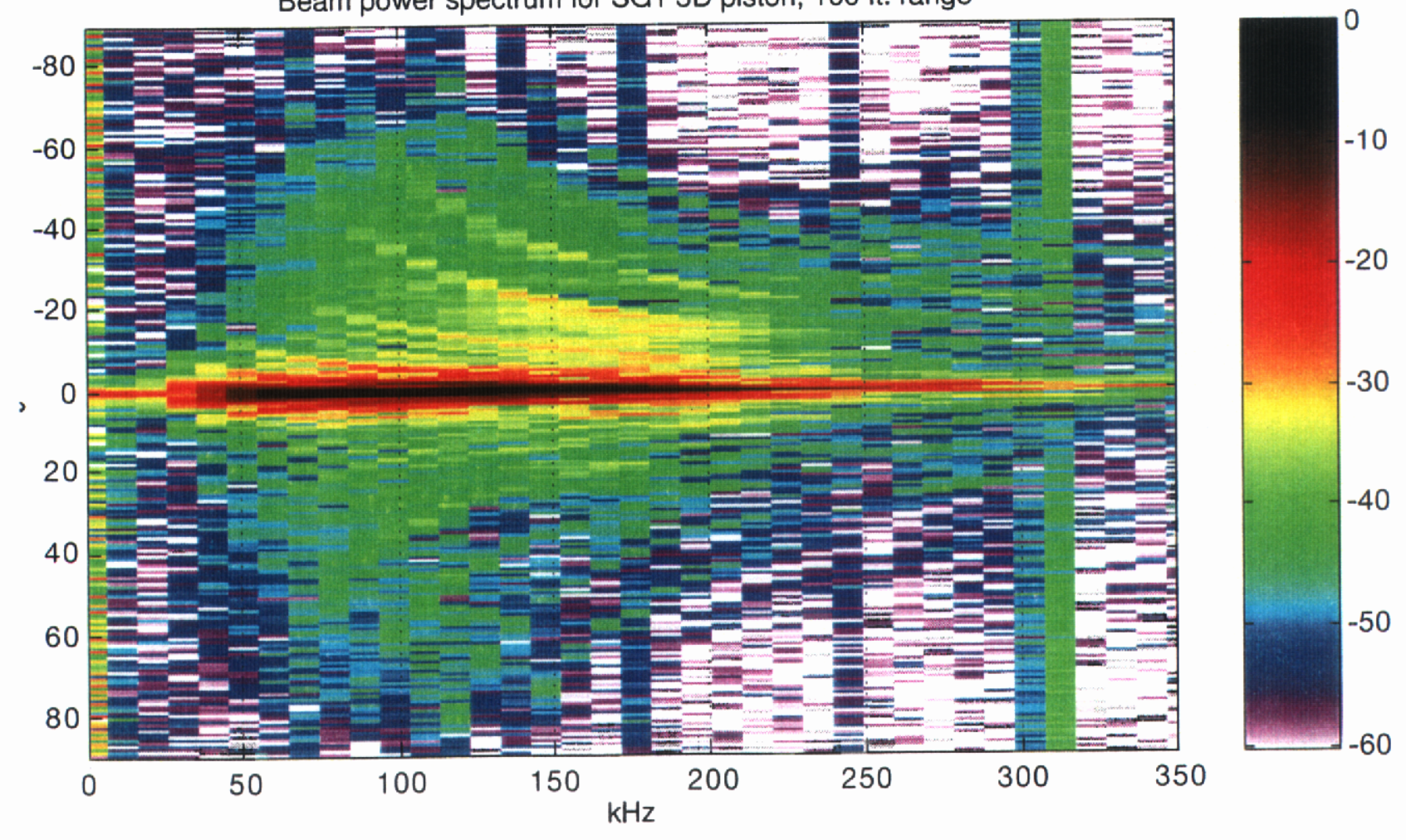


Beam power spectrum for MPS3, 200 yd. range
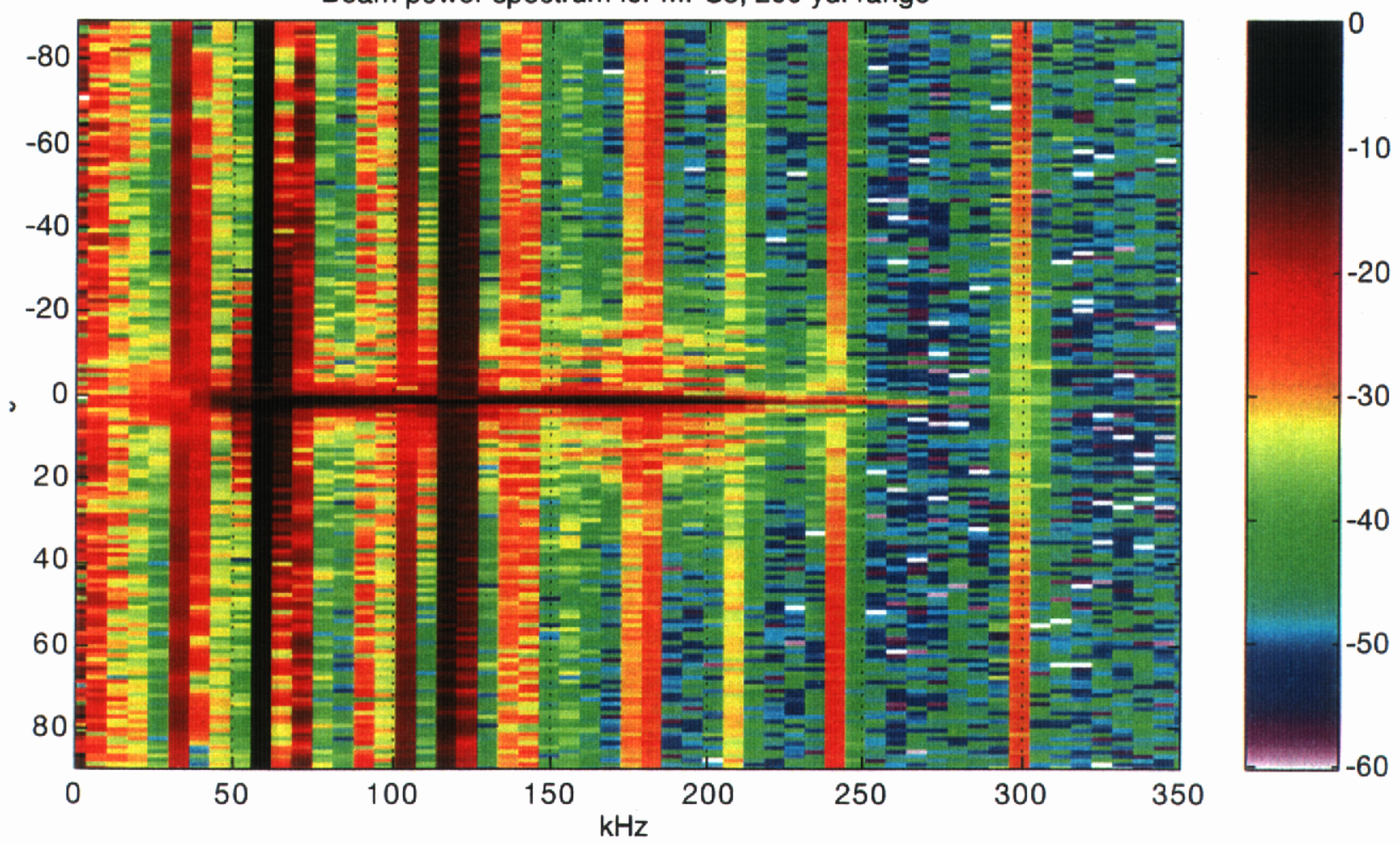

Beam power spectrum for MPS3 piston, 200 yd. range

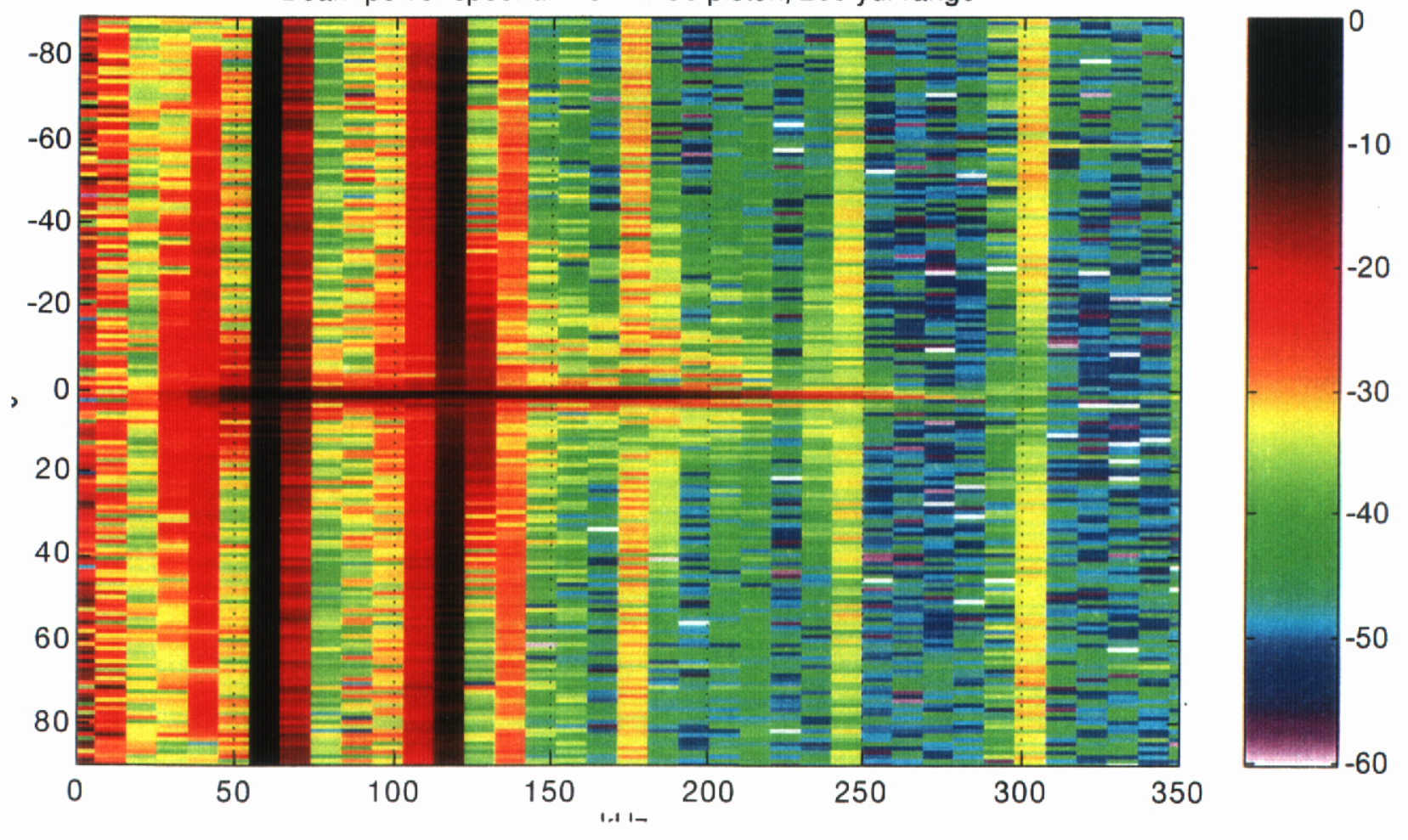


Beam power spectrum for MPS3 compressed, 200 yd. range
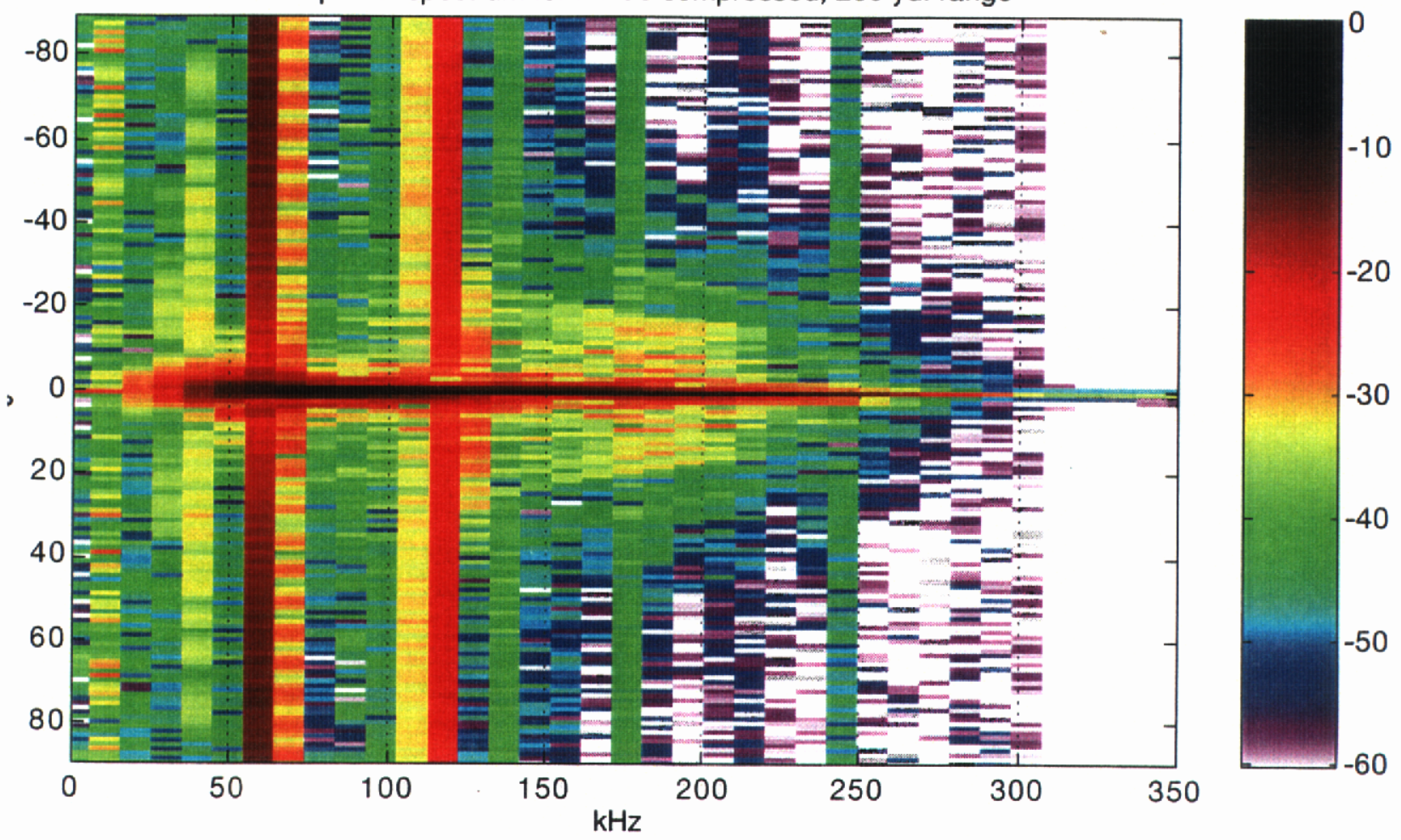

Beam power spectrum for MPS3 piston compressed, $200 \mathrm{yd}$. range
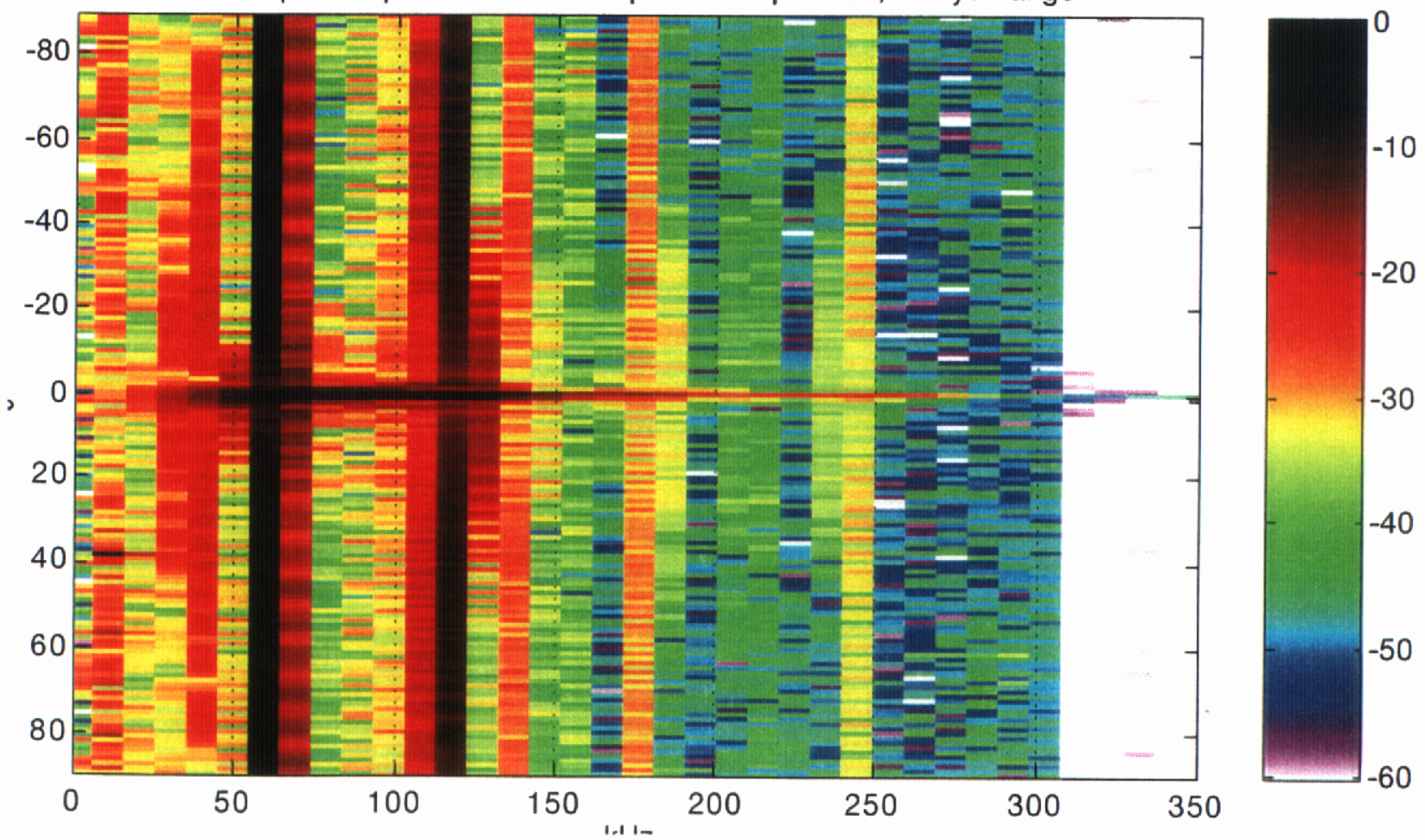
Beam power spectrum for $20 \mathrm{kHz}$ tone burst, $100 \mathrm{ft}$. range
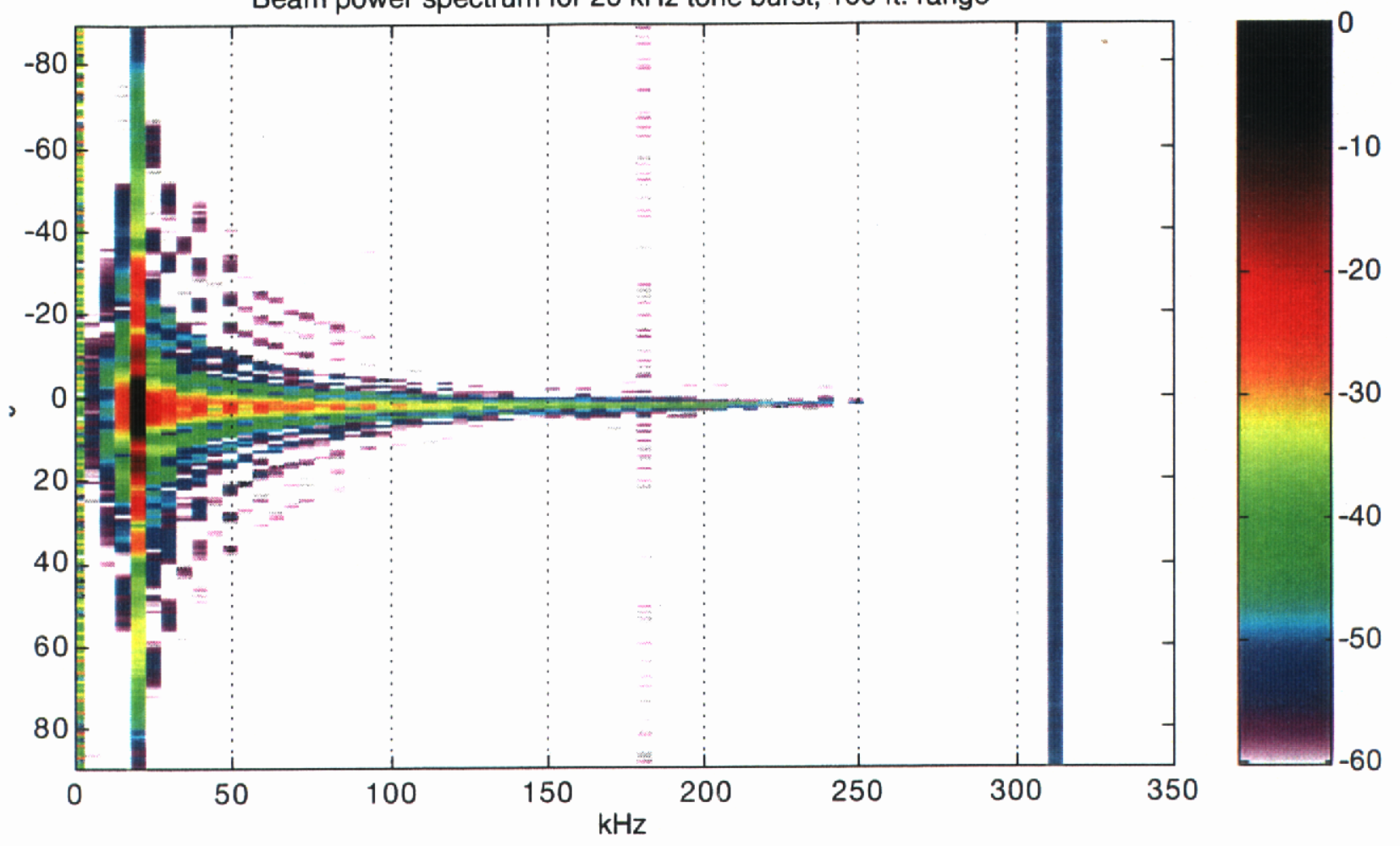

Beam power spectrum for $20 \mathrm{kHz}$ tone burst, $200 \mathrm{yd}$. range
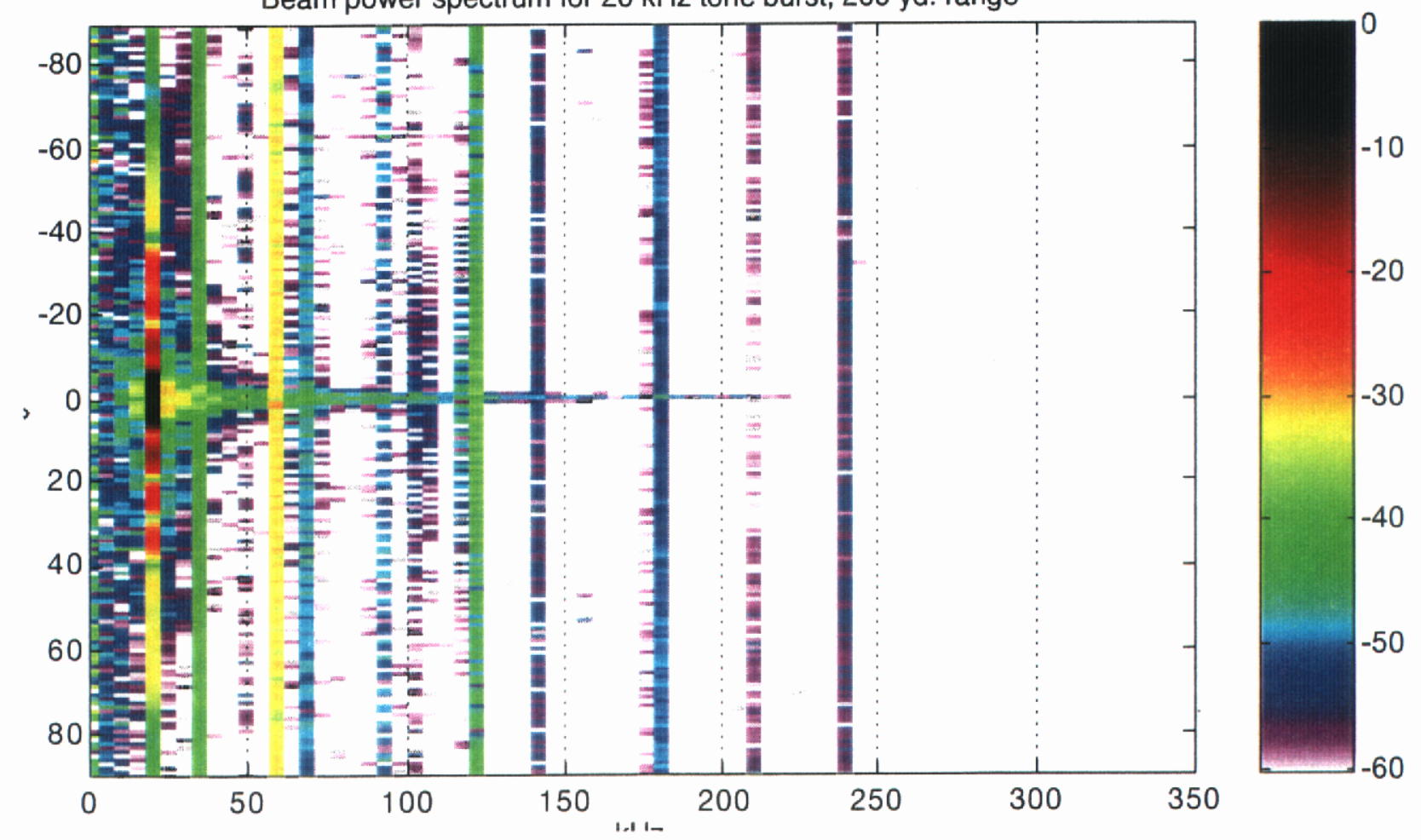

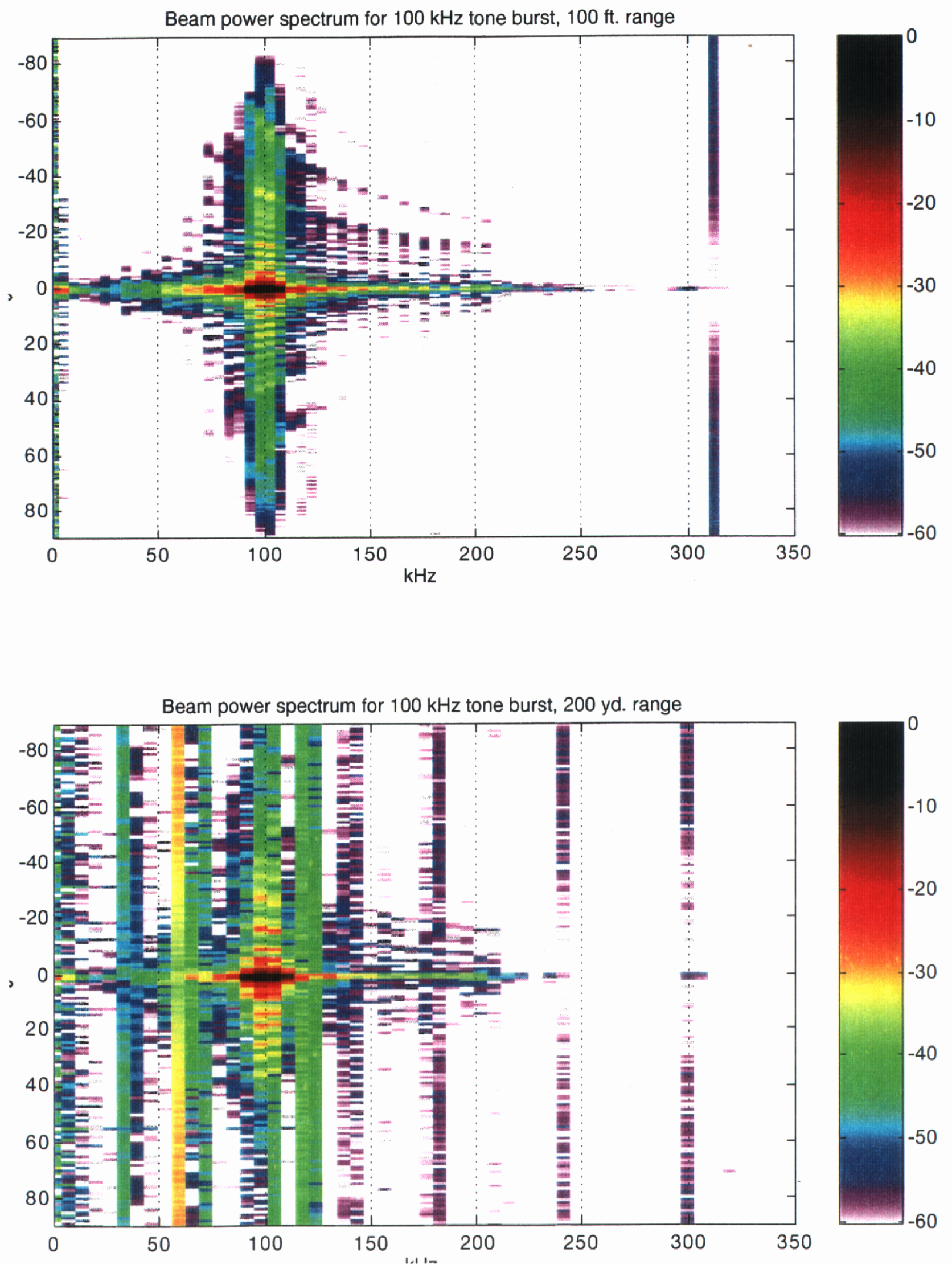
Beam power spectrum for $240 \mathrm{kHz}$ tone burst, $100 \mathrm{ft}$. range

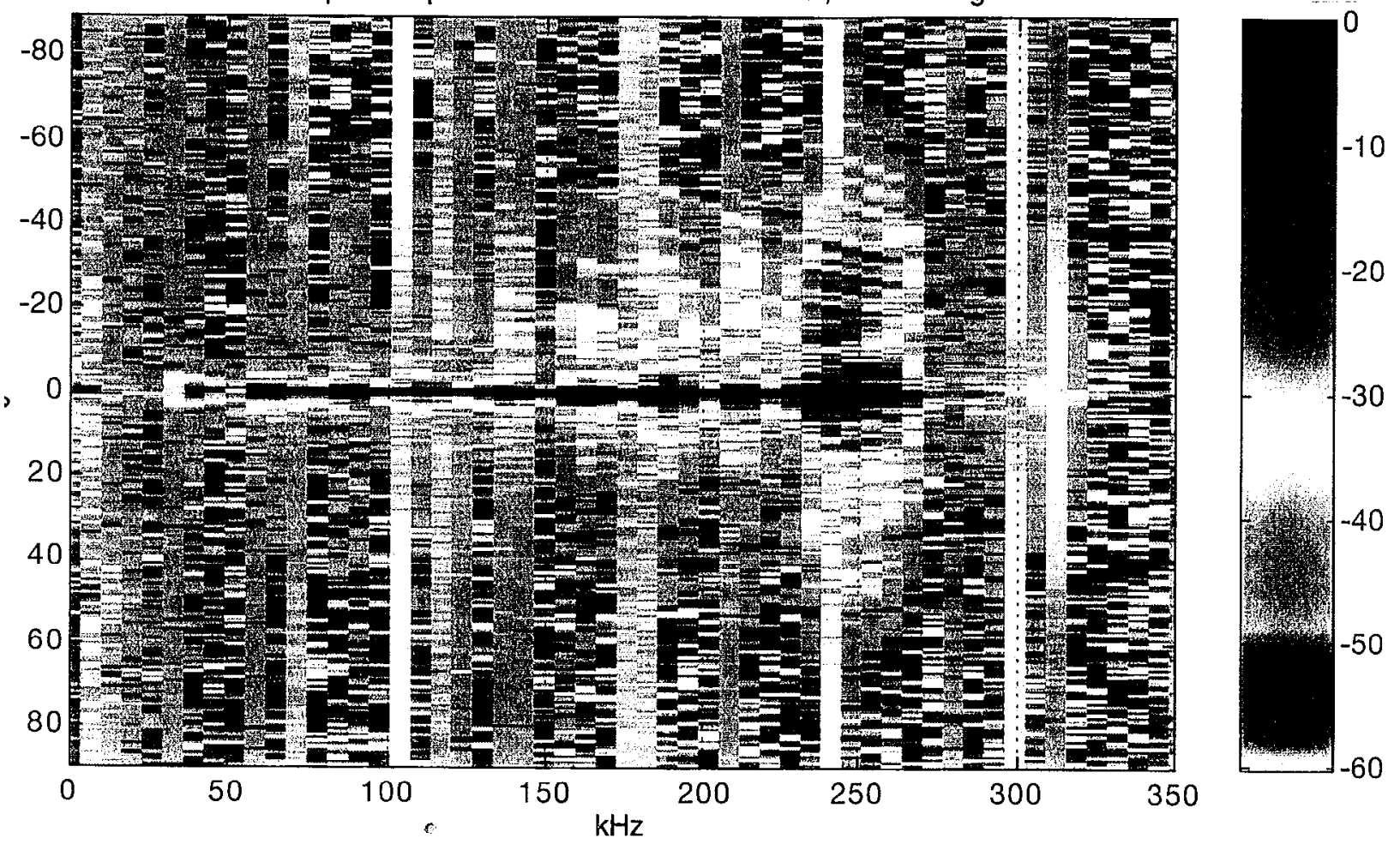

Beam power spectrum for $240 \mathrm{kHz}$ tone burst, $200 \mathrm{yd}$. range

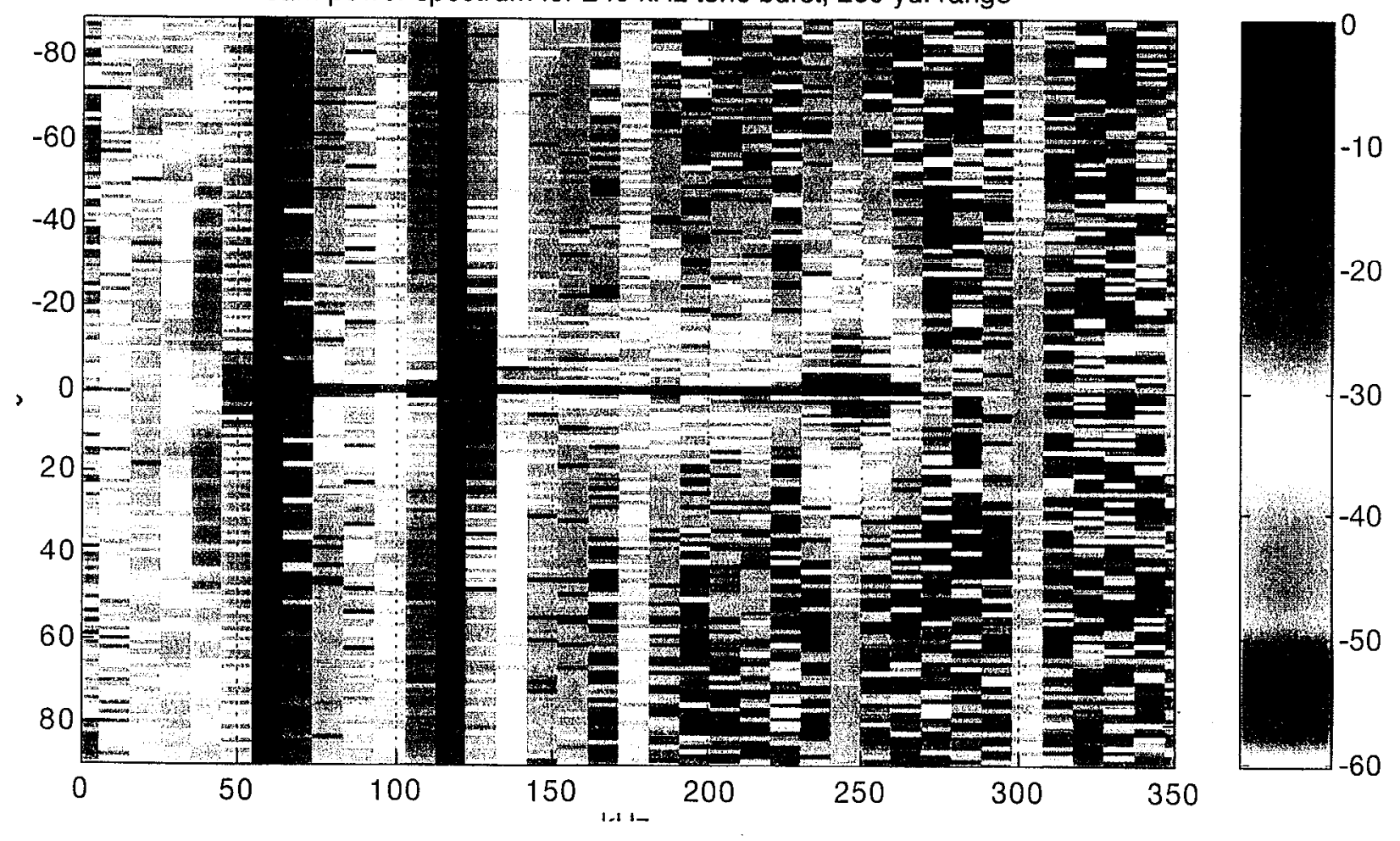


Beam power spectrum for $240 \mathrm{kHz}$ tone burst, $100 \mathrm{ft}$. range
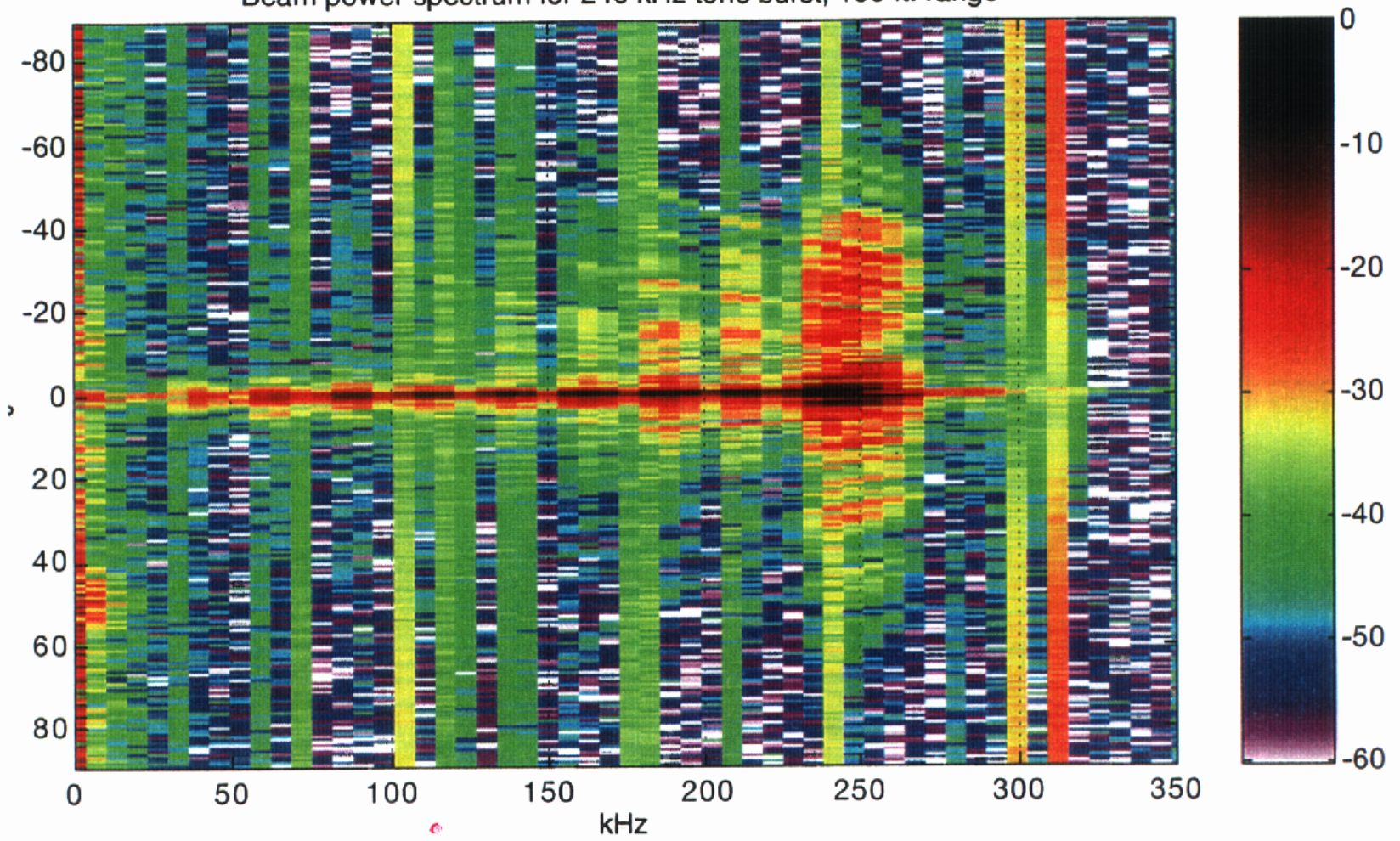

Beam power spectrum for $240 \mathrm{kHz}$ tone burst, $200 \mathrm{yd}$. range
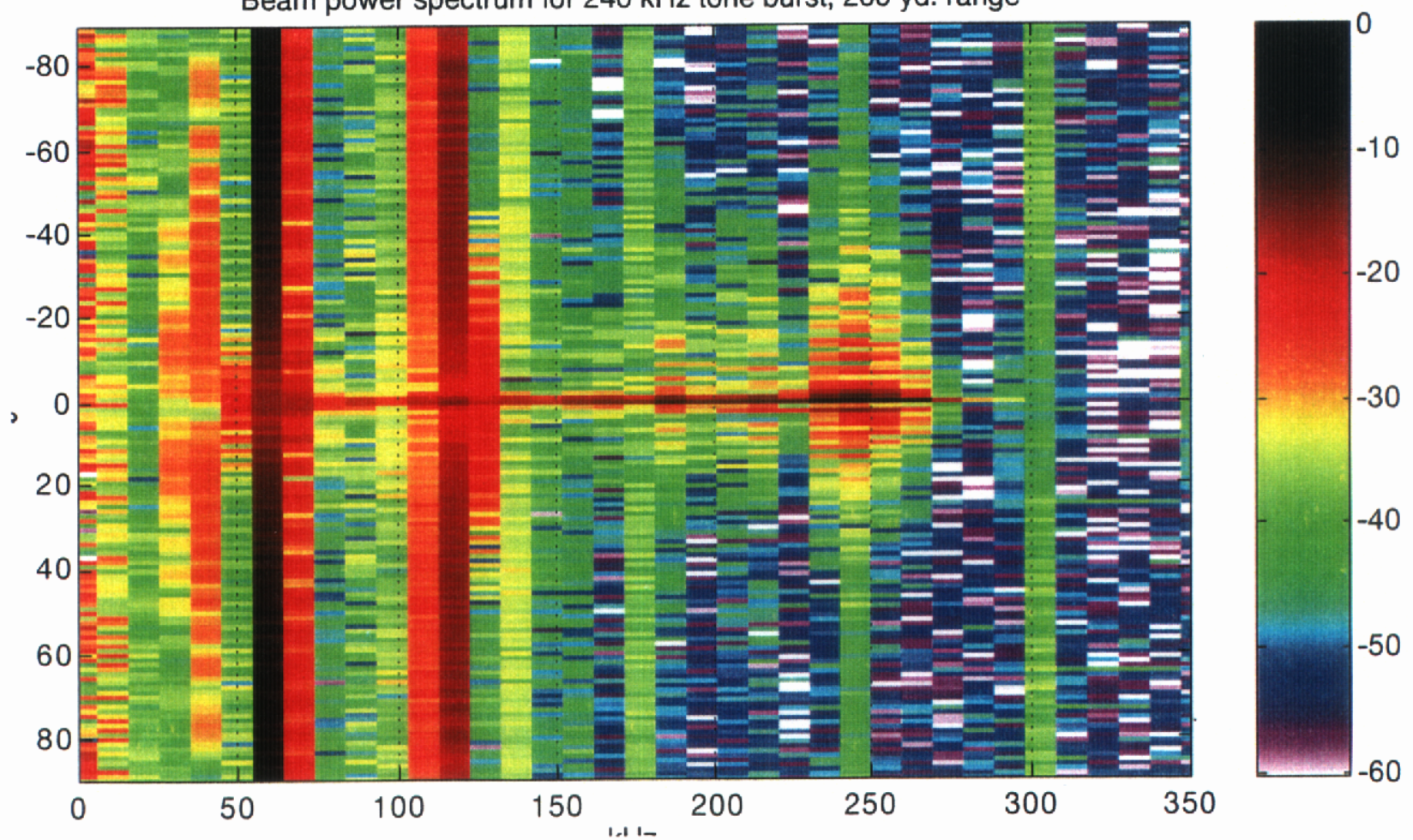


\section{Appendix E1. Linear angle beam patterns}

The following figures are the total power of the various pulses, including the tone bursts, as functions of angle. The data is the normalized value of the time integral of the square of the received field at the 2 distances of the experiment, 100 and 600 feet. Each data set includes the full half space field from -90 to +90 degrees, and the center of the field expanded for -5 to +5 degrees. The horizontal axis is in $\mathrm{dB}$ for all cases.

The data shows the results of both a coarse scan, lots of points spaced relatively far apart encompassing -90 to +90 degrees relative to the array axis, and a fine scan, lots of points spaced closer together over a smaller angle range. The data also shows the results of the raw beam, the compressed beam, as described in the main body of the report, and the recompressed beam after deconvolution. 
Beam pattern for MPS1 NC, $100 \mathrm{ft}$. range

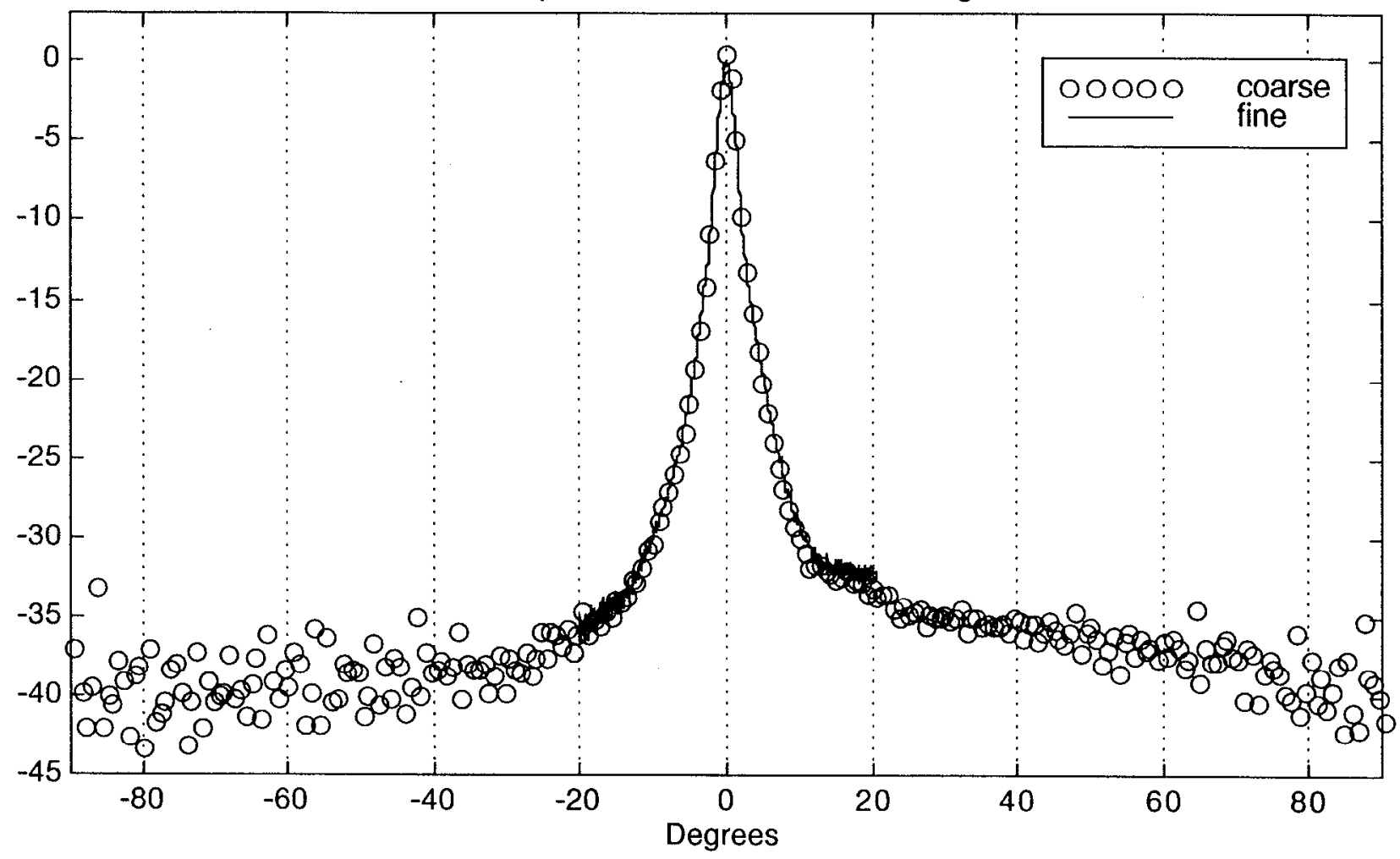

Figure E1-1. Beam pattern for MPS1 with no compensation at a 100 foot range. Above is -90 to +90 degrees, below is -5 to +5 degrees. Amplitudes are in $\mathrm{dB}$.

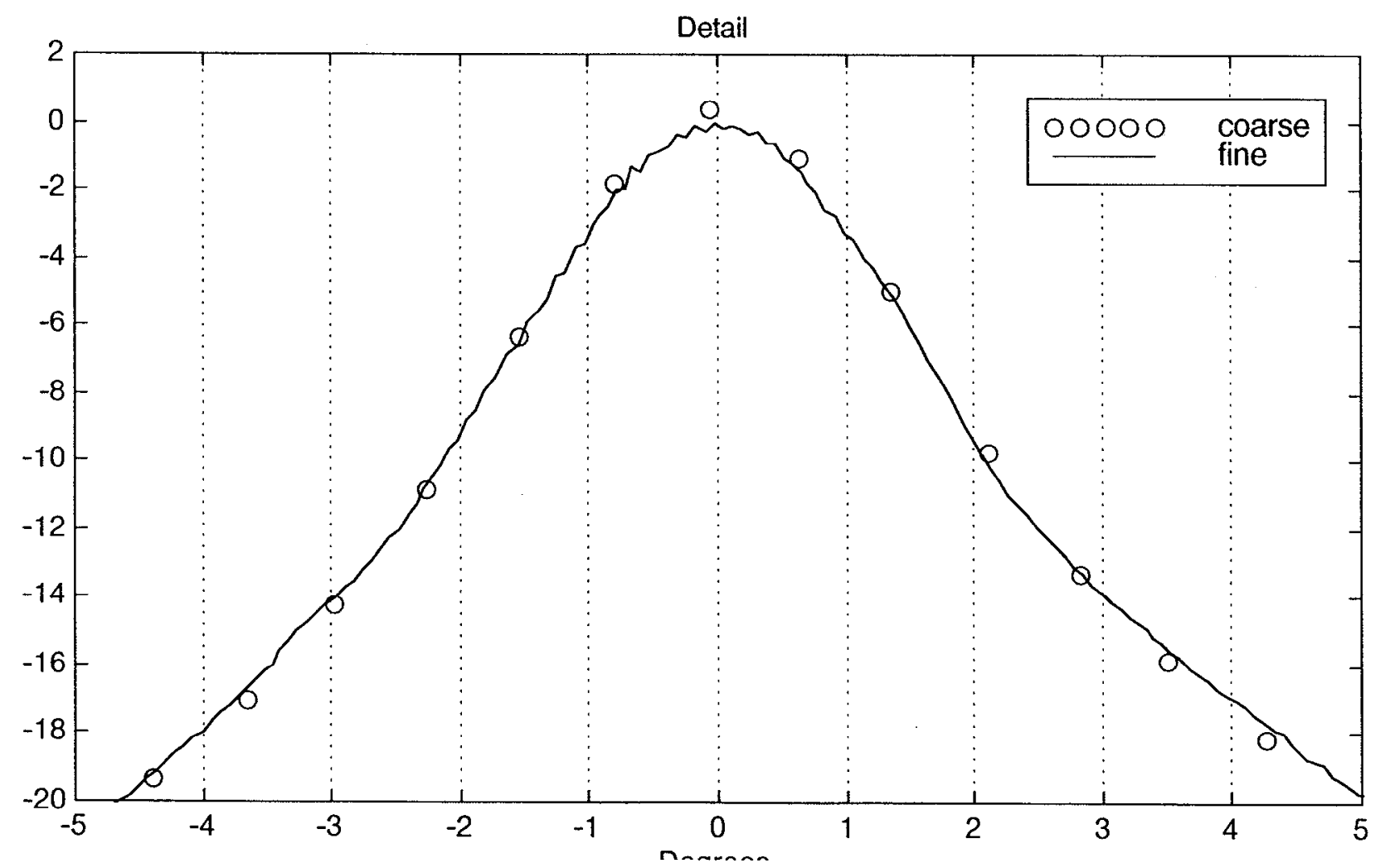


Beam pattern for MPS1 2D, $100 \mathrm{ft}$. range

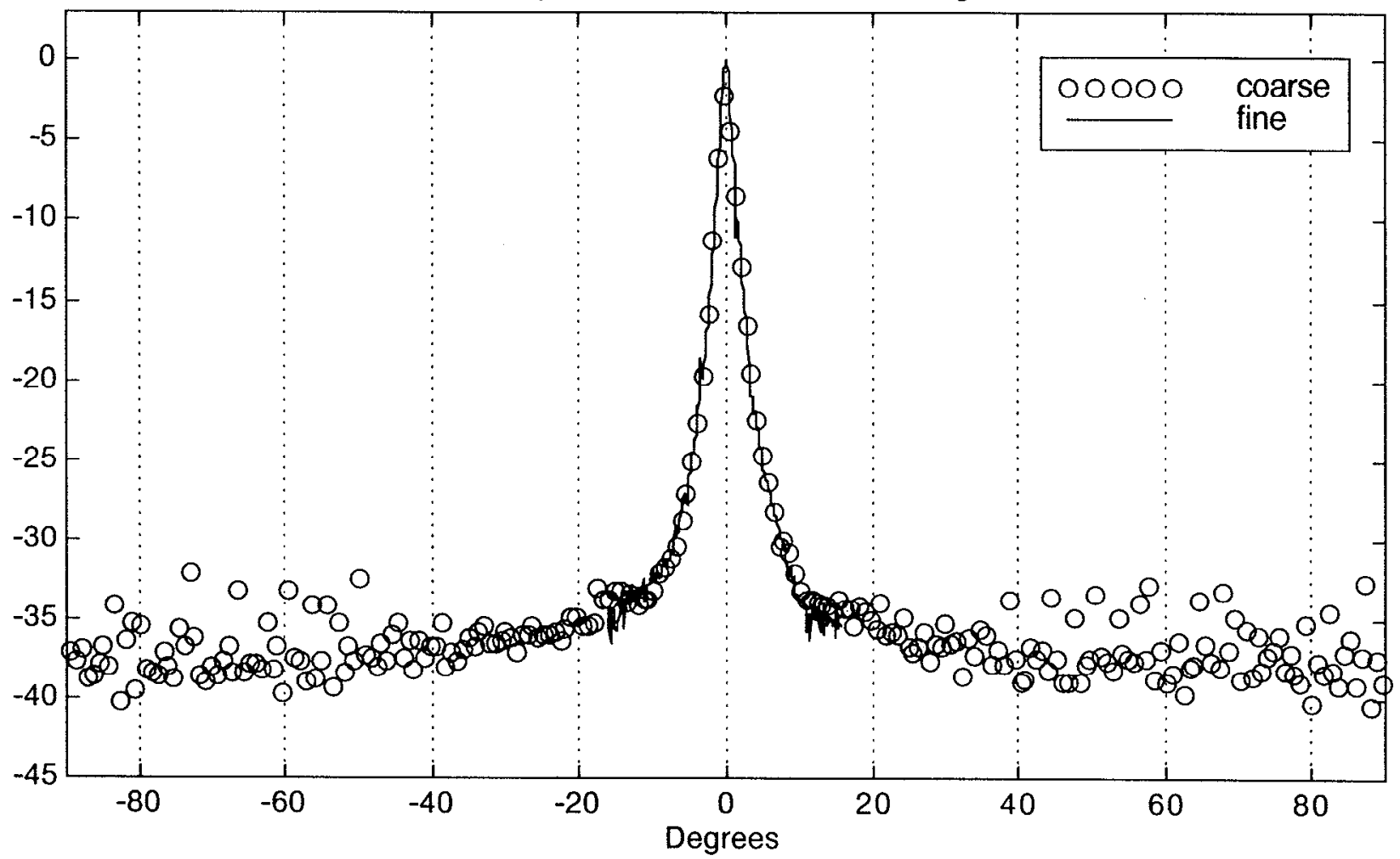

Figure E1-2. Beam pattern for MPS1 with $2^{\text {nd }}$ derivative compensation at a 100 foot range. Above is -90 to +90 degrees, below is -5 to +5 degrees. Amplitudes are in $\mathrm{dB}$.

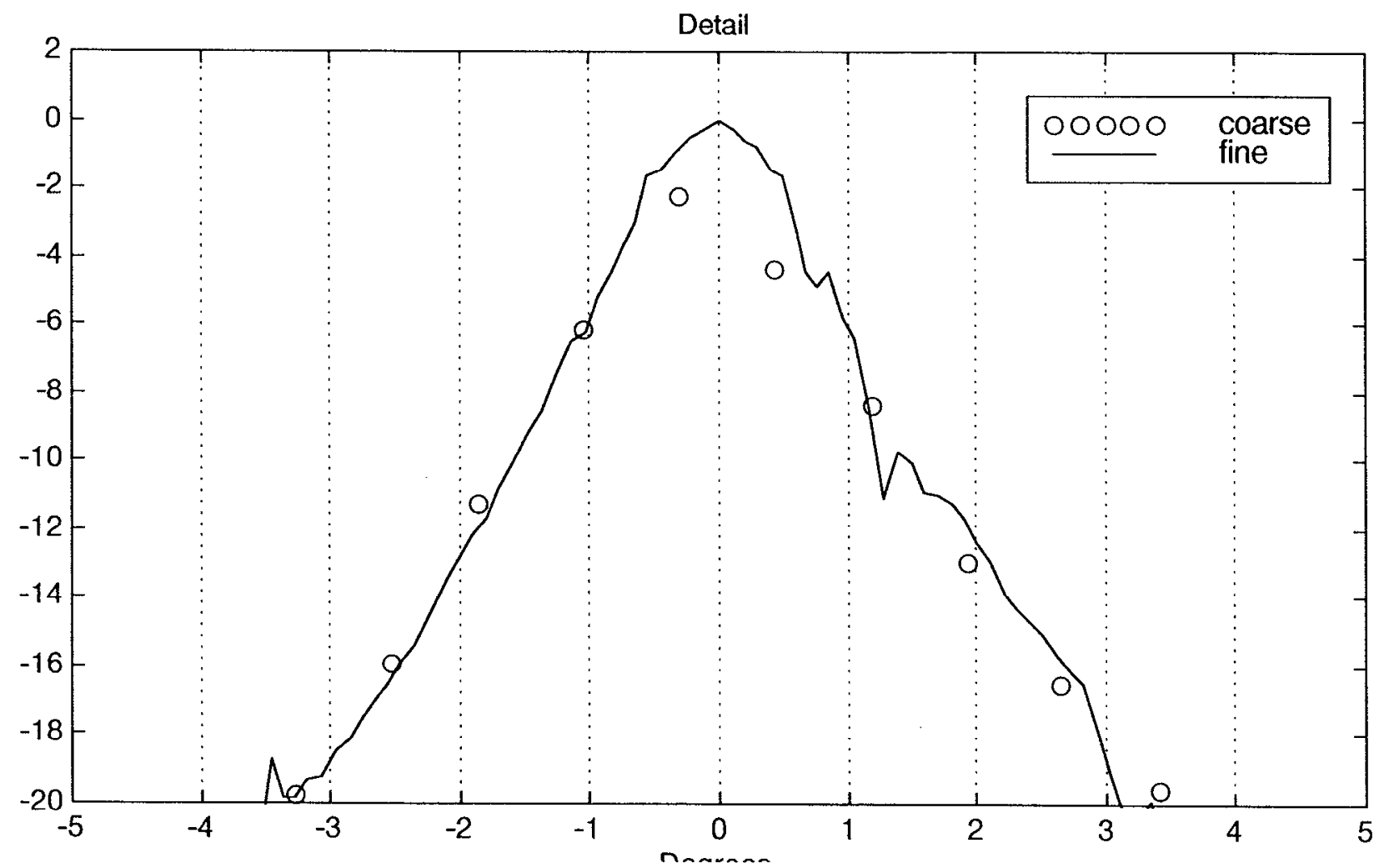


Beam pattern for MPS1 2D piston, $100 \mathrm{ft}$. range

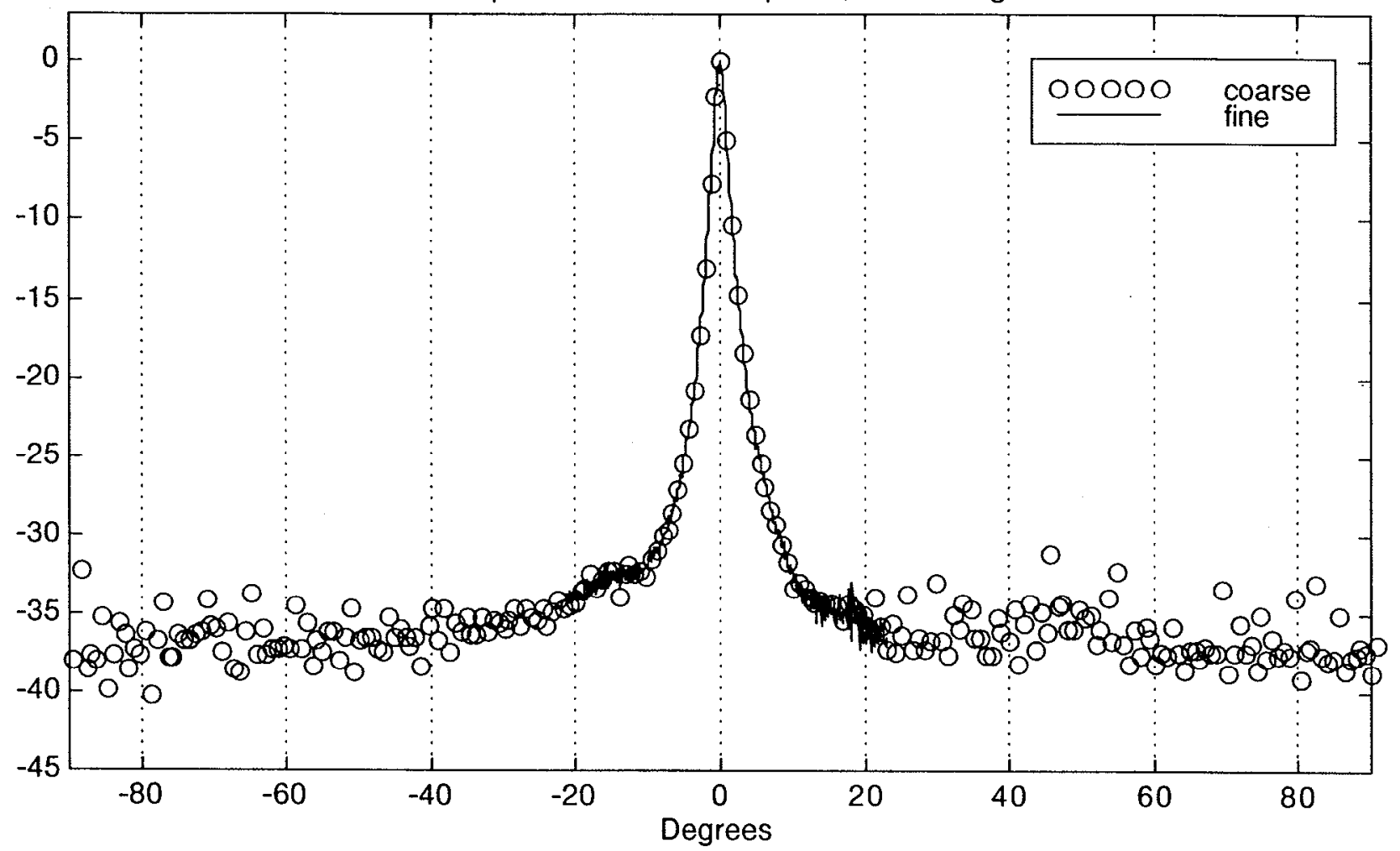

Figure E1-3. Beam pattern for MPS1 piston drive with $2^{\text {nd }}$ derivative compensation at a 100 foot range. Above is -90 to +90 degrees, below is -5 to +5 degrees. Amplitudes are in $\mathrm{dB}$.

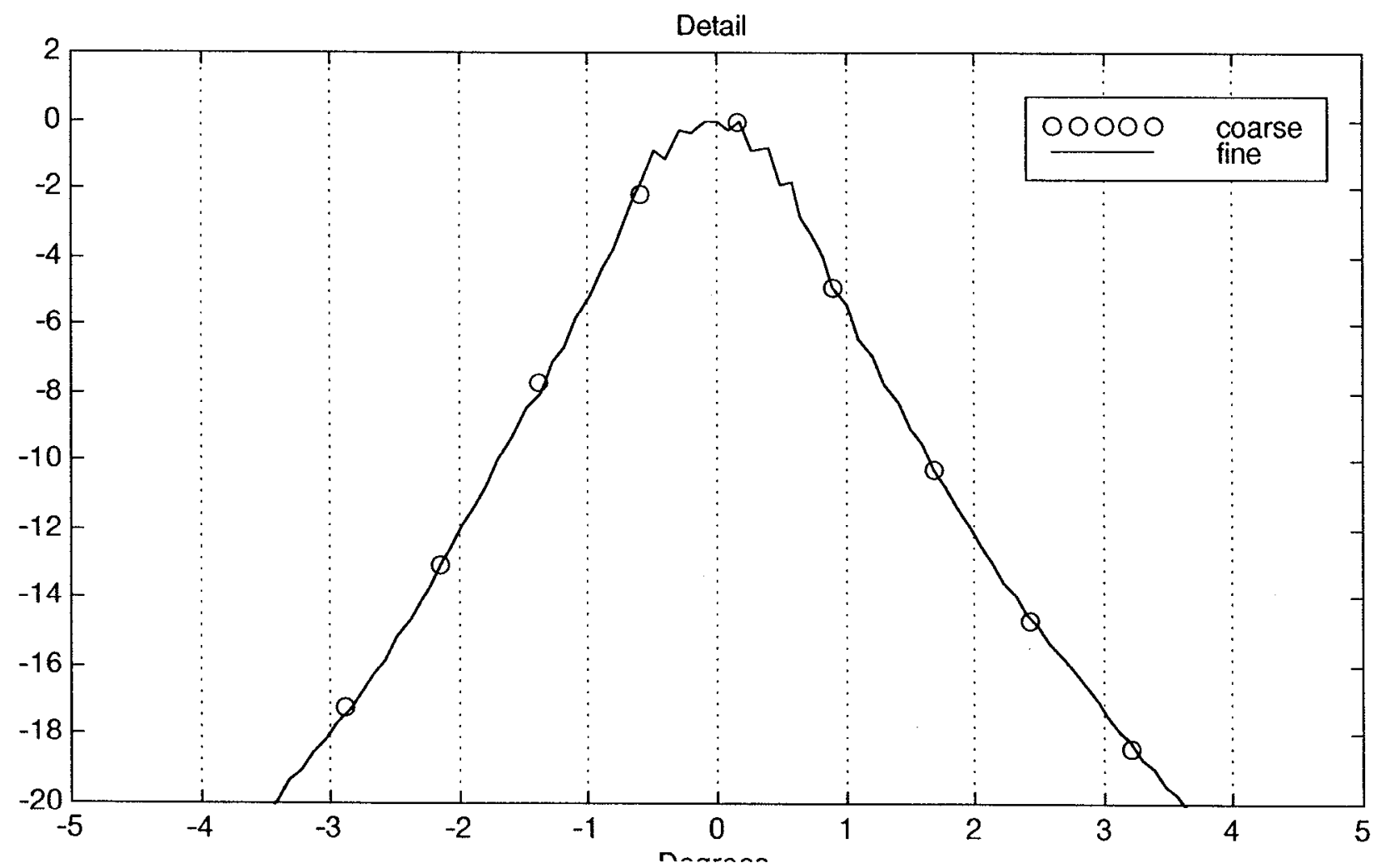


Beam pattern for MPS1 3D, $100 \mathrm{ft}$. range

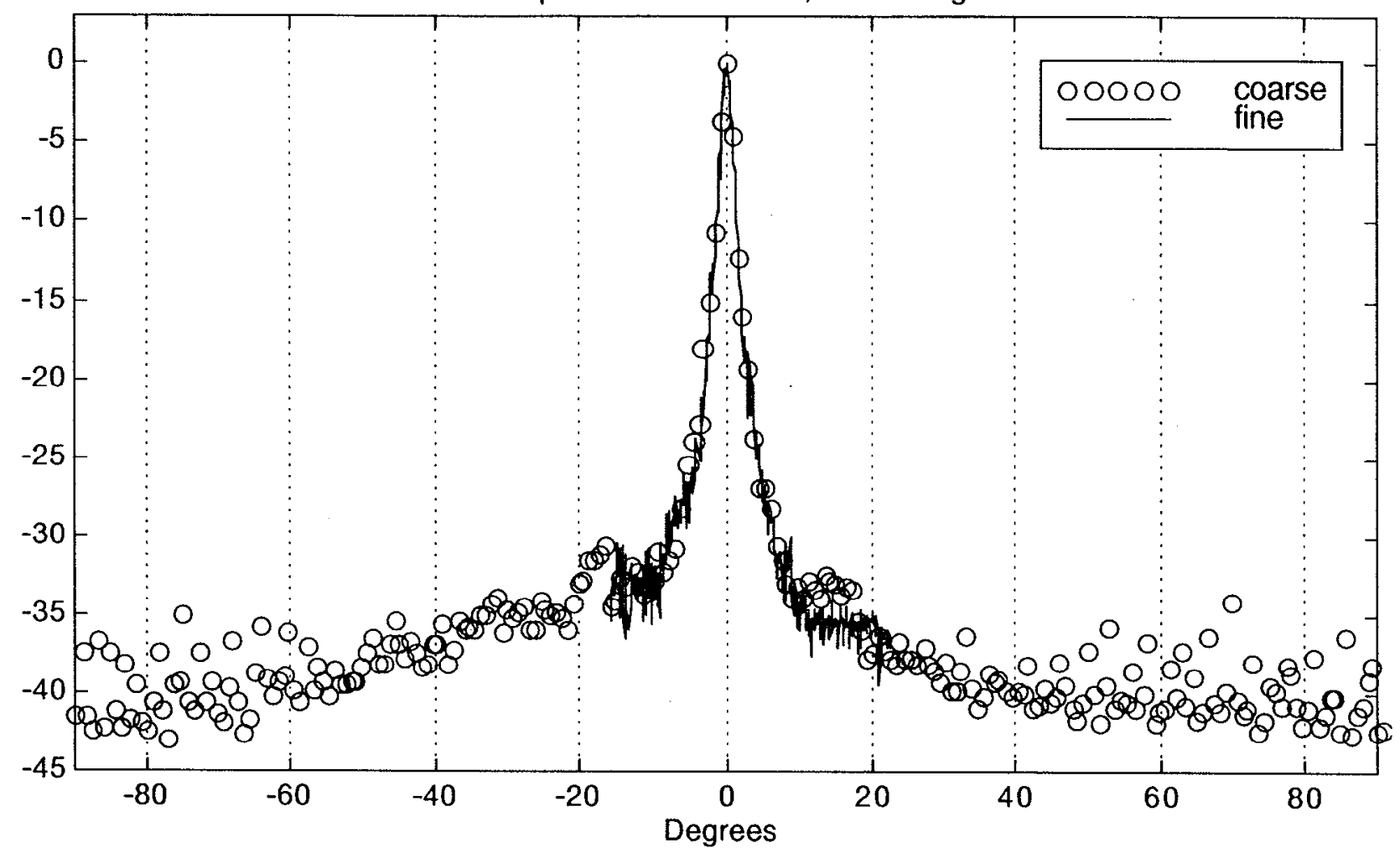

Figure E1-4. Beam pattern for MPS1 with $3^{\text {rd }}$ derivative compensation at a 100 foot range. Above is -90 to +90 degrees, below is -5 to +5 degrees. Amplitudes are in $\mathrm{dB}$.

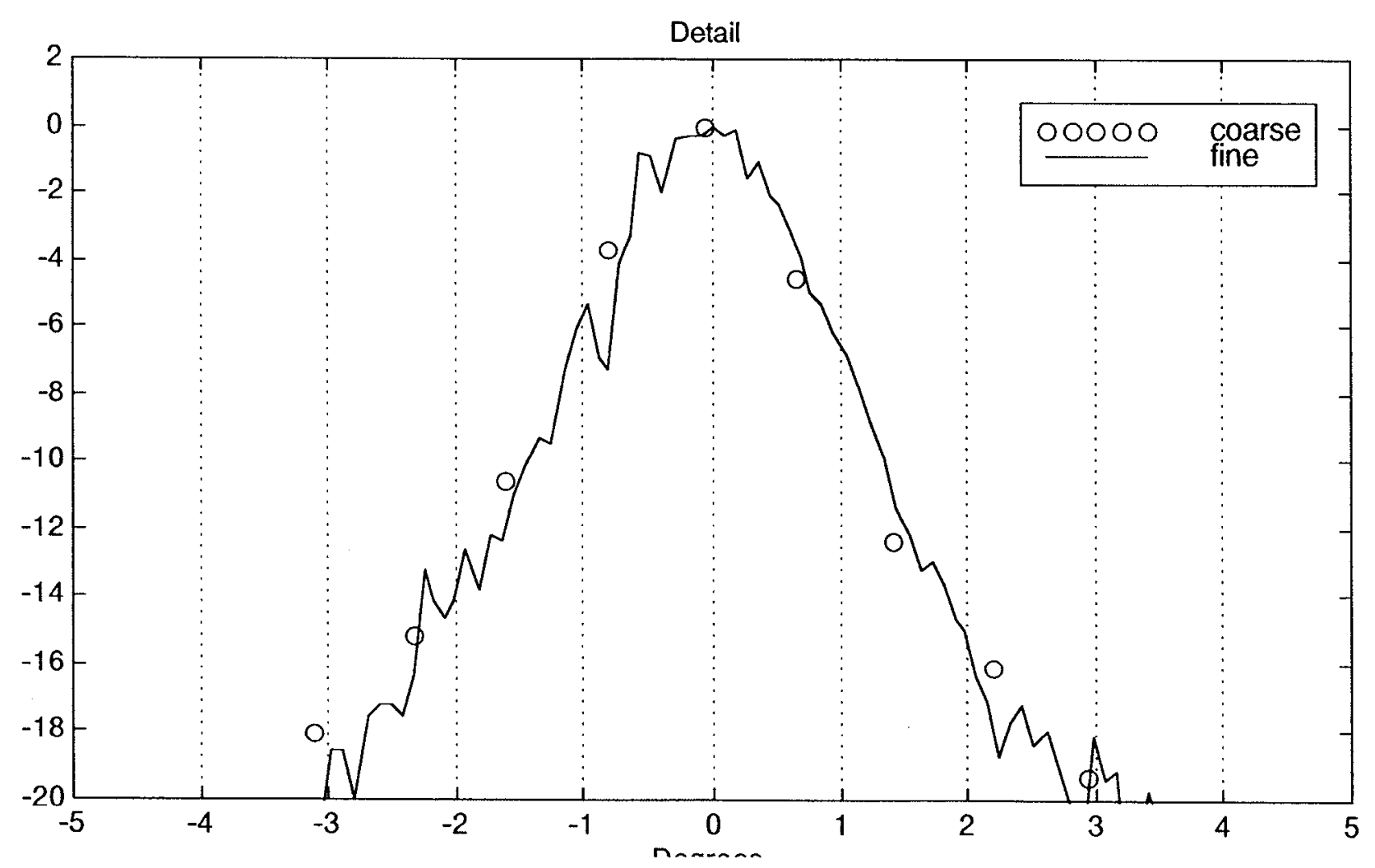


Beam pattern for MPS1 3D piston, $100 \mathrm{ft}$. range

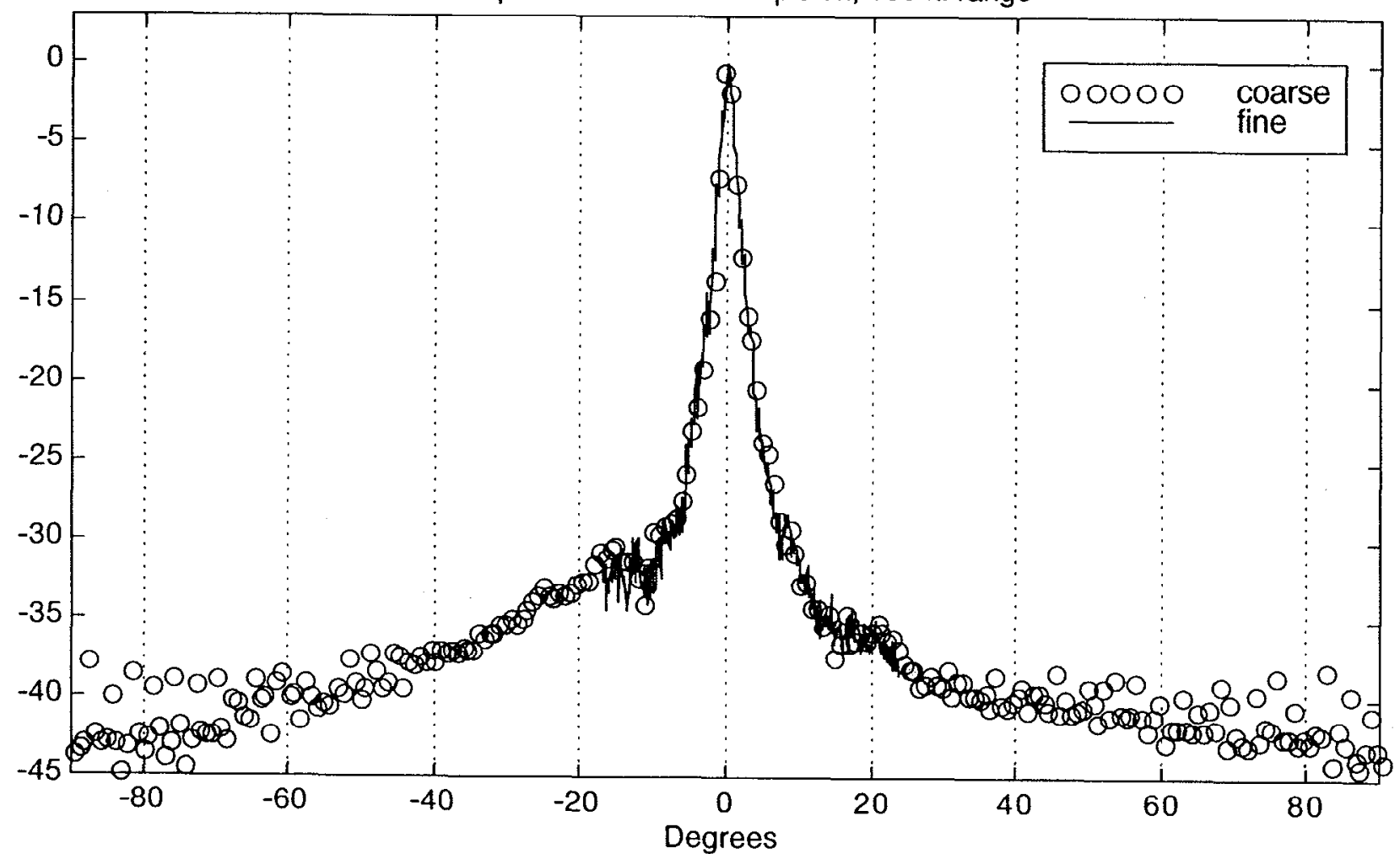

Figure E1-5. Beam pattern for MPS1 piston drive with $3^{\text {rd }}$ derivative compensation at a 100 foot range. Above is -90 to +90 degrees, below is -5 to +5 degrees. Amplitudes are in $\mathrm{dB}$.

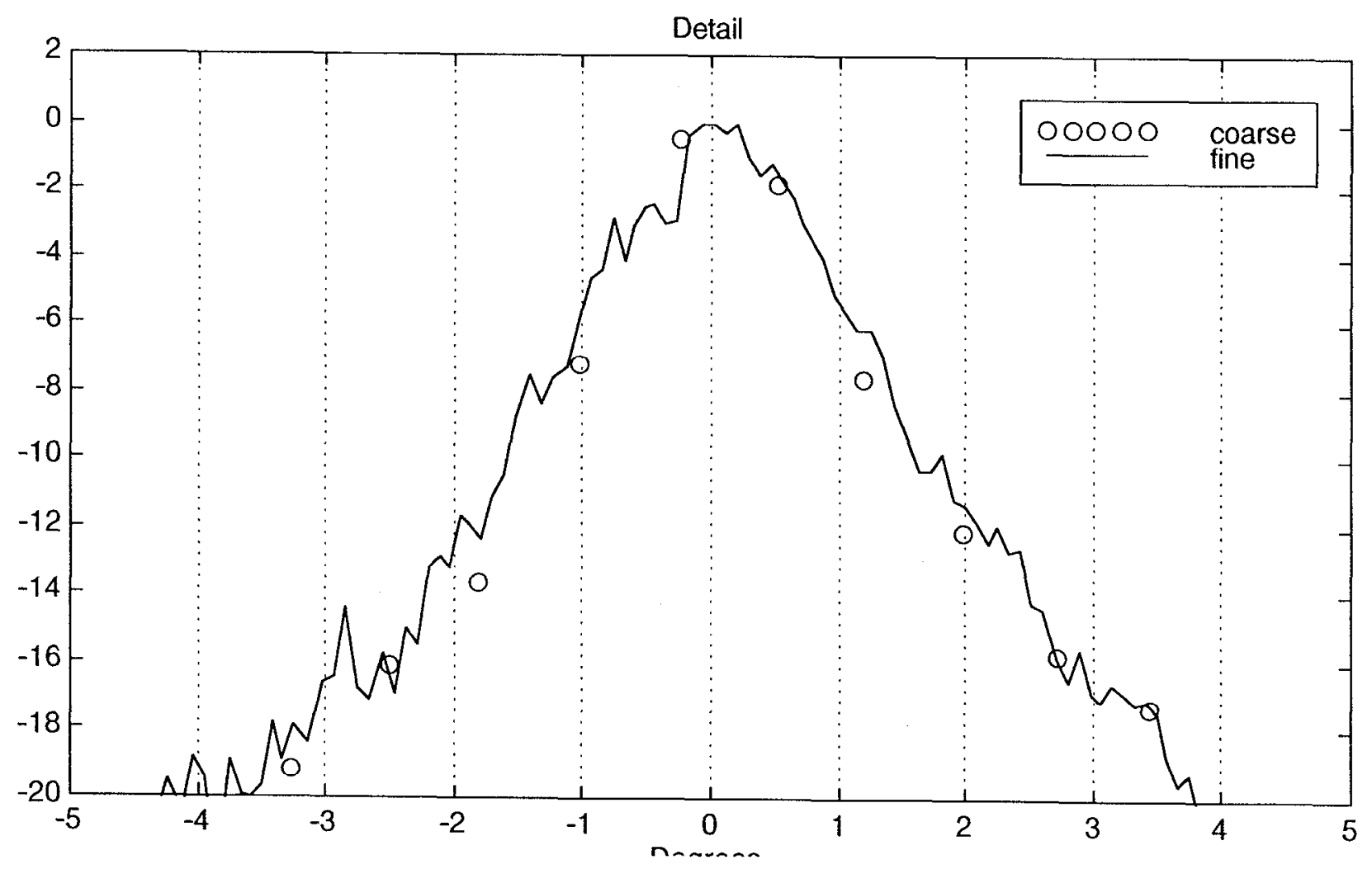


Beam pattern for MPS1 2D, 200 yd. range

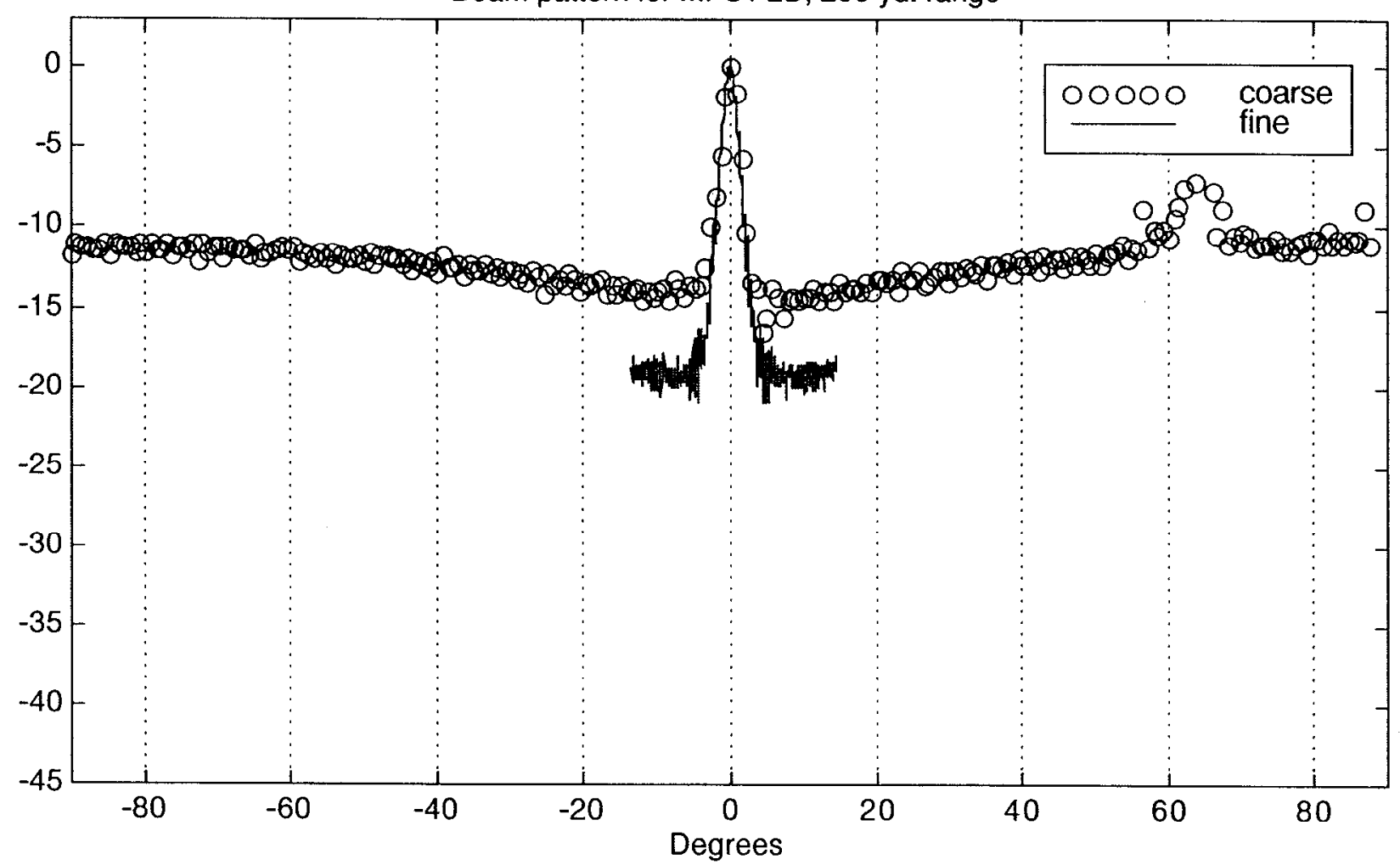

Figure E1-6. Beam pattern for MPS1 pulse with 2nd derivative compensation at a 600 foot range. Above is -90 to +90 degrees, below is -5 to +5 degrees. Amplitudes are in $\mathrm{dB}$.

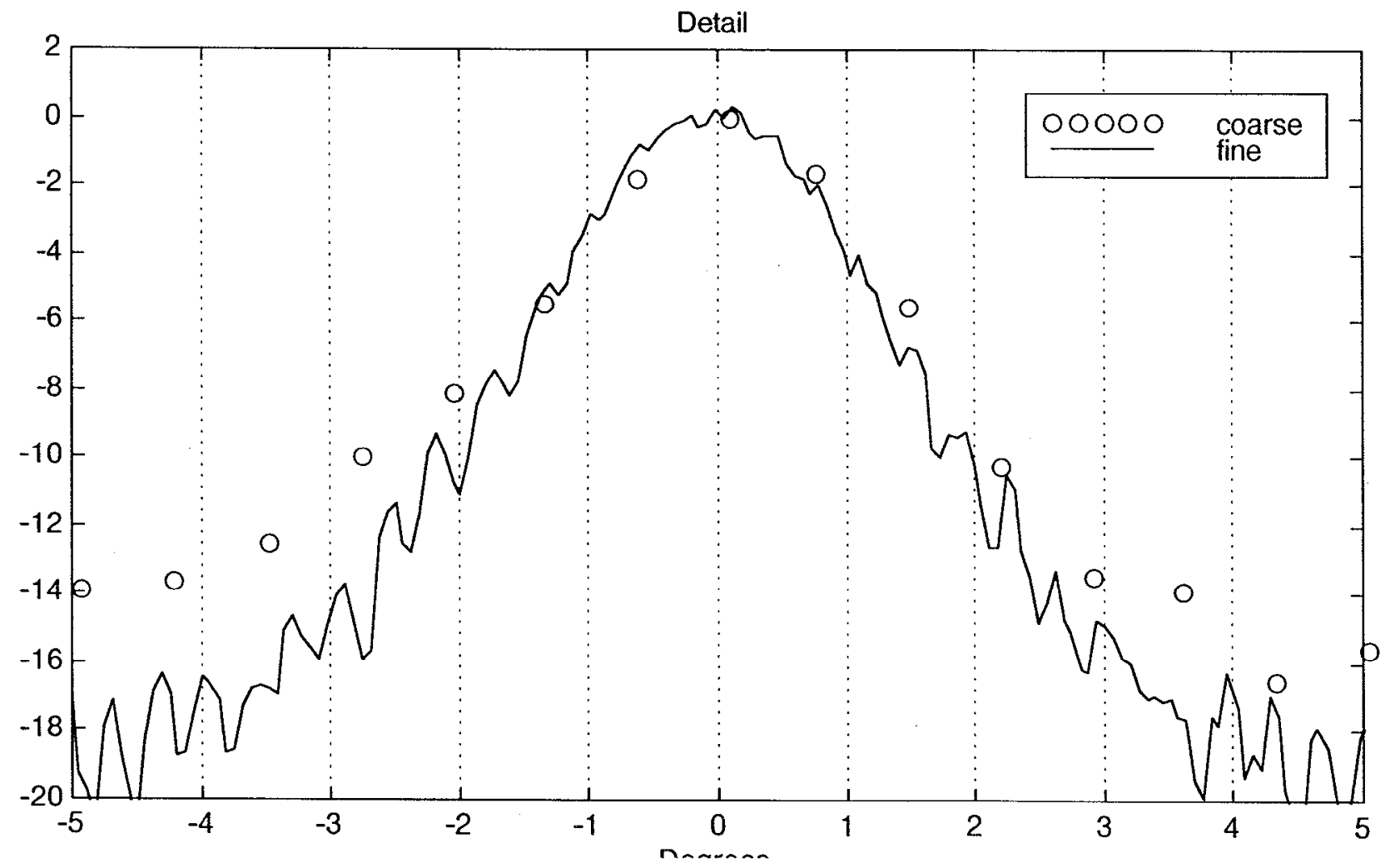


Beam pattern for MPS1 2D piston, 200 yd. range

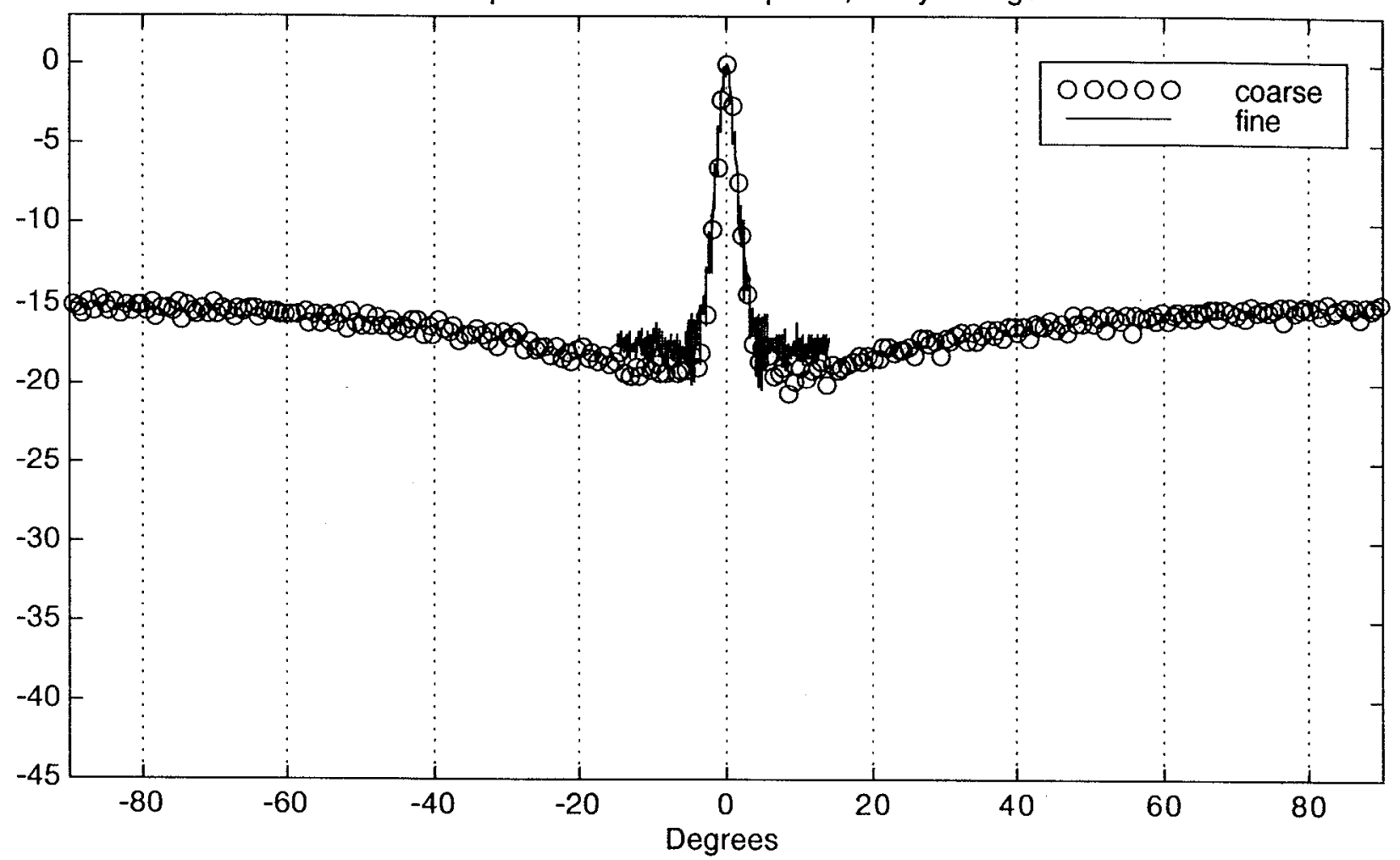

Figure E1-7. Beam pattern for MPS1 piston drive with 2nd derivative compensation at a 600 foot range. Above is -90 to +90 degrees, below is -5 to +5 degrees. Amplitudes are in $\mathrm{dB}$.

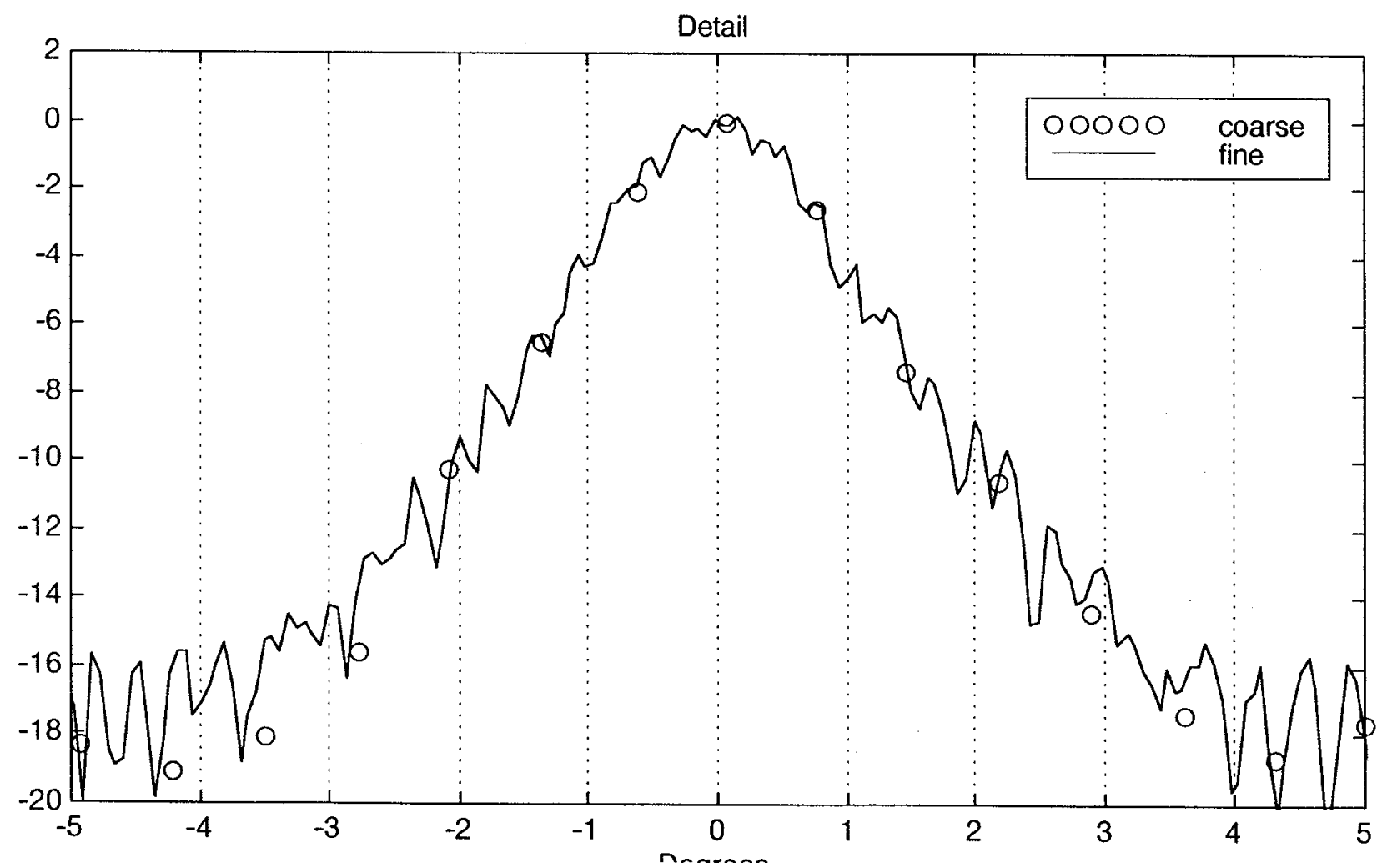


Beam pattern for MPS1 stretched, $100 \mathrm{ft}$. range

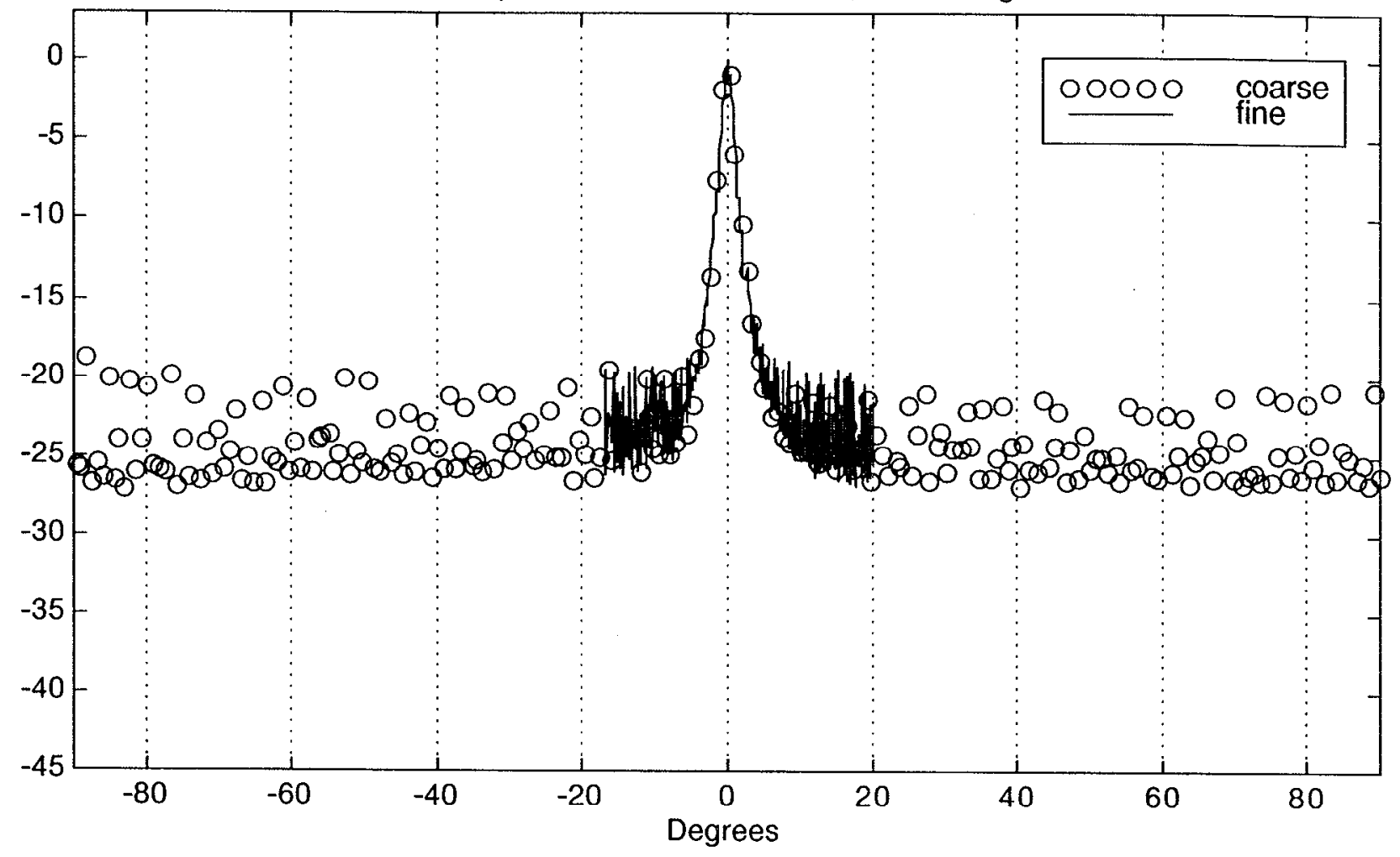

Figure E1-8. Beam pattern for MPS1 frequency stretched pulse with no compensation at a 100 foot range. Above is -90 to +90 degrees, below is -5 to +5 degrees. Amplitudes are in $\mathrm{dB}$.

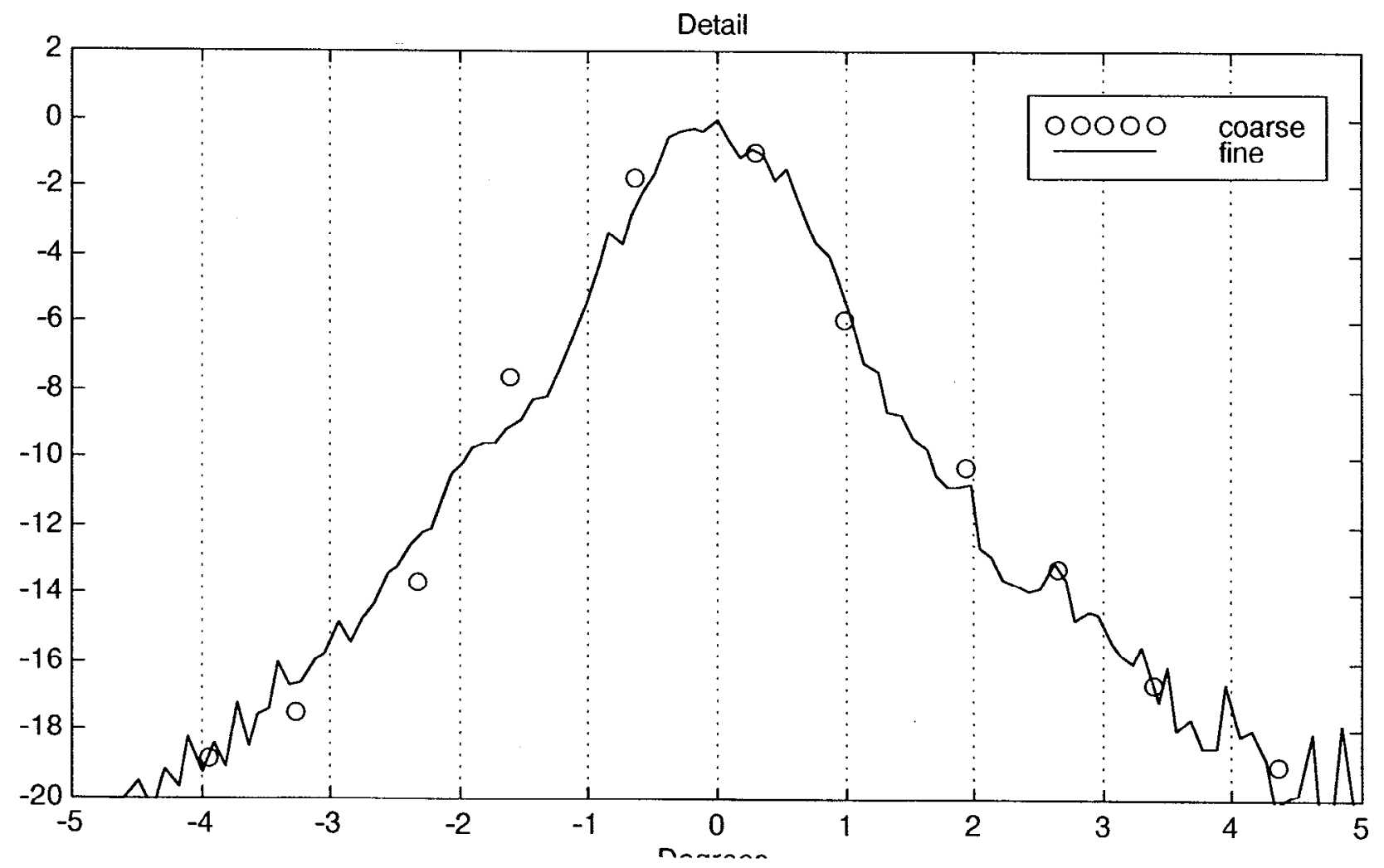




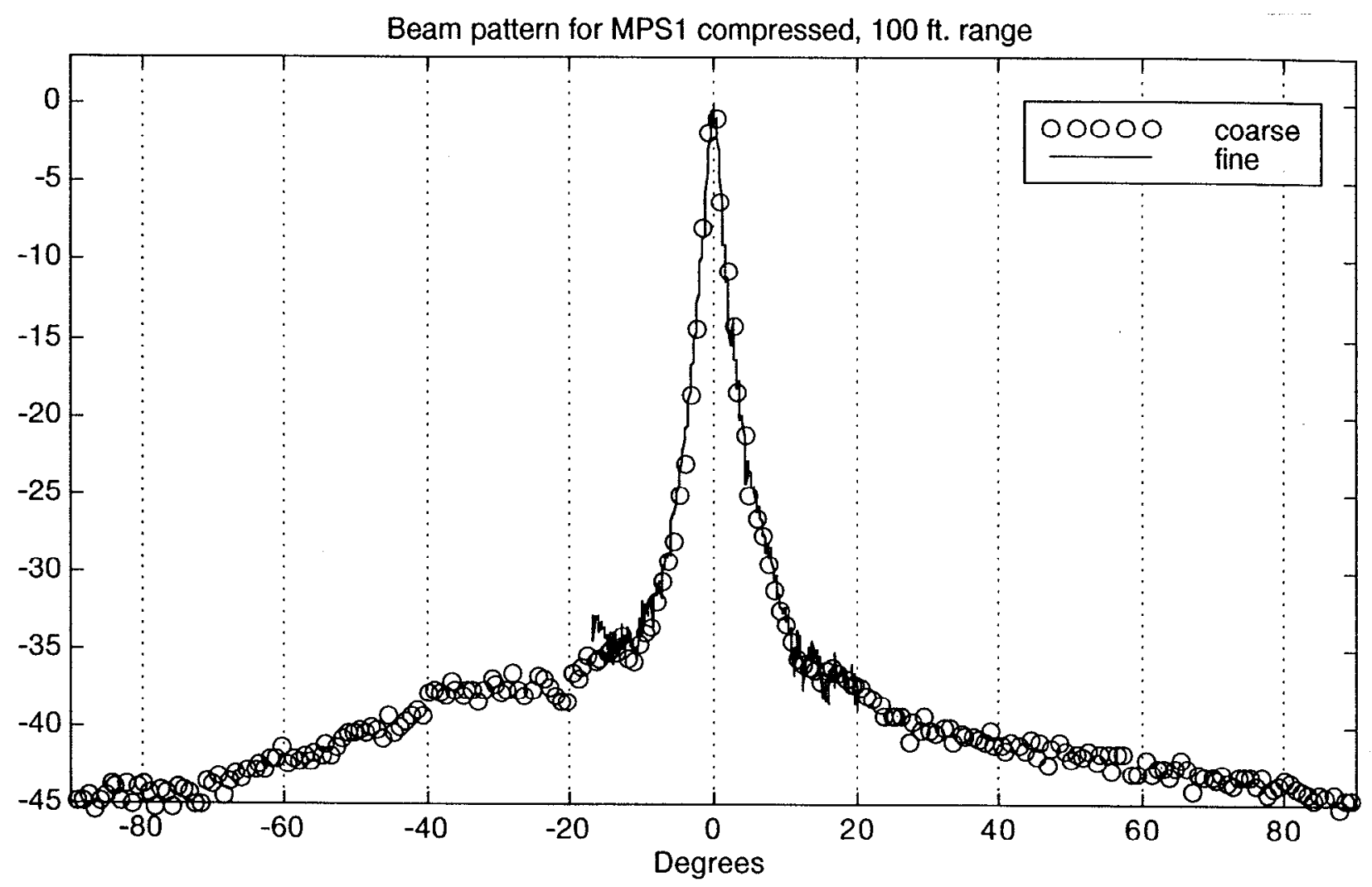

Figure E1-9. Beam pattern for MPS1 stretched and recompressed with no compensation at a 100 foot range. Above is -90 to +90 degrees, below is -5 to +5 degrees. Amplitudes are in $\mathrm{dB}$.

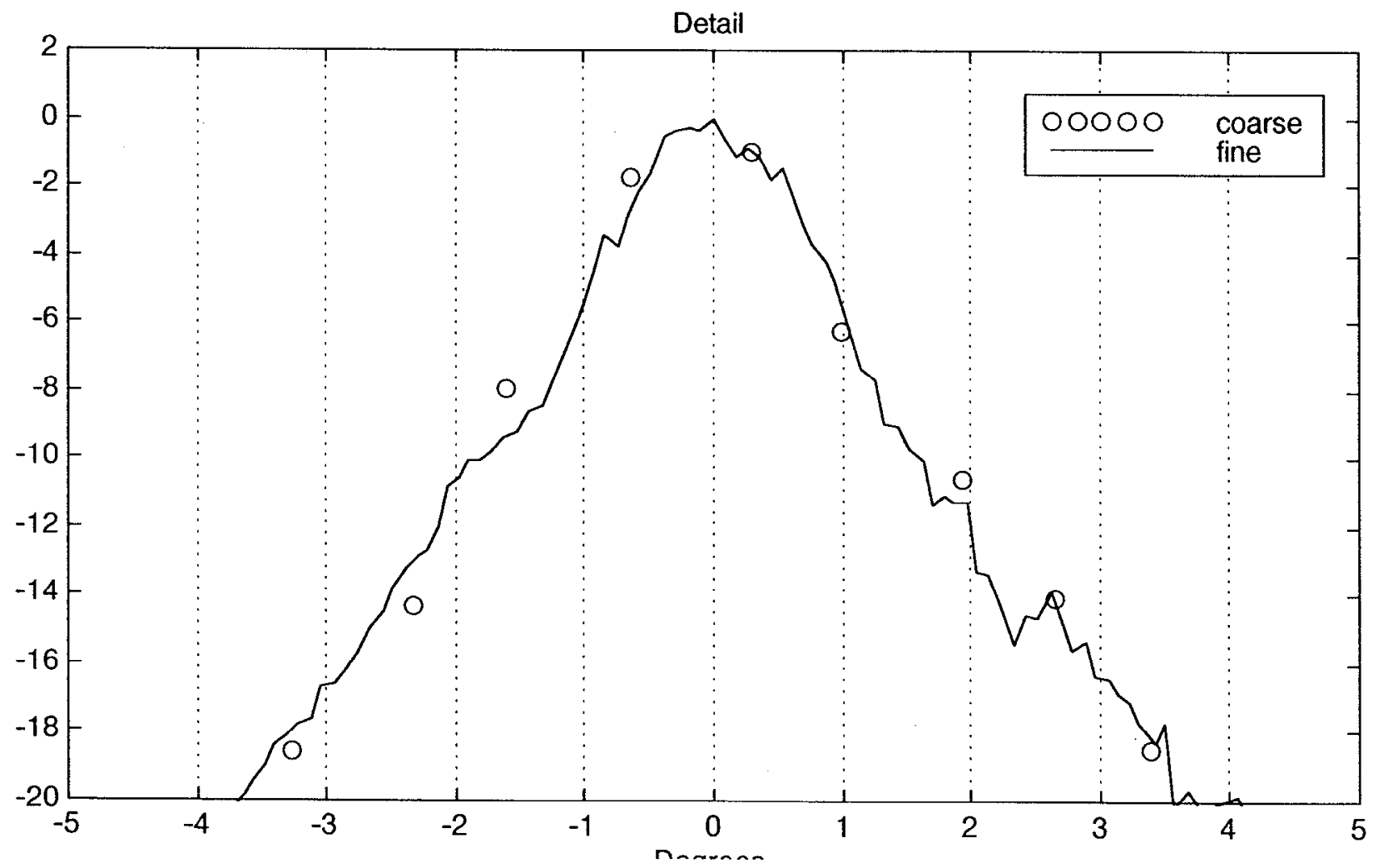


Beam pattern for MPS1 stretched piston, $100 \mathrm{ft}$. range

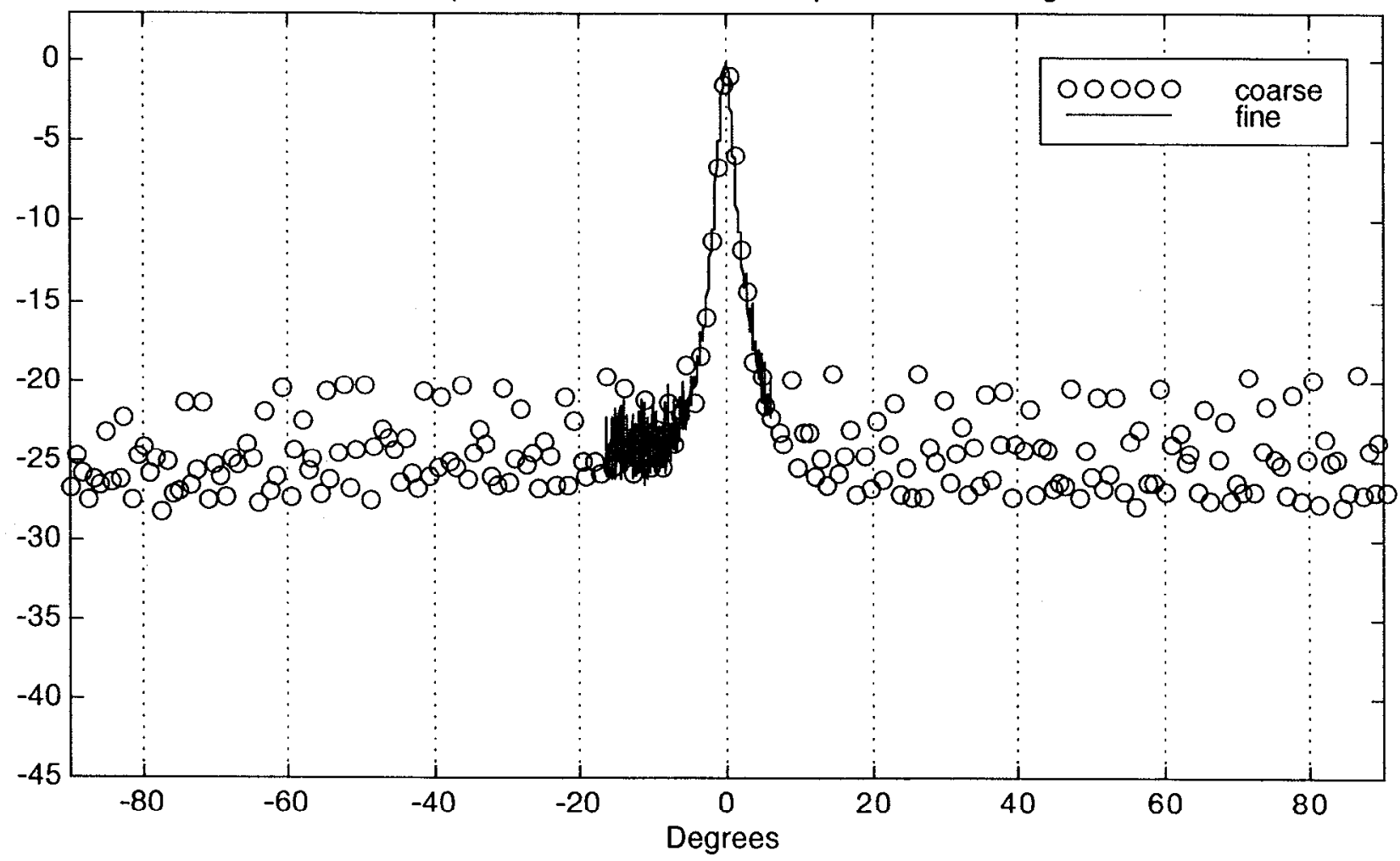

Figure E1-10. Beam pattern for MPS1 stretched piston drive with no compensation at a 100 foot range. Above is -90 to +90 degrees, below is -5 to +5 degrees. Amplitudes are in $\mathrm{dB}$.

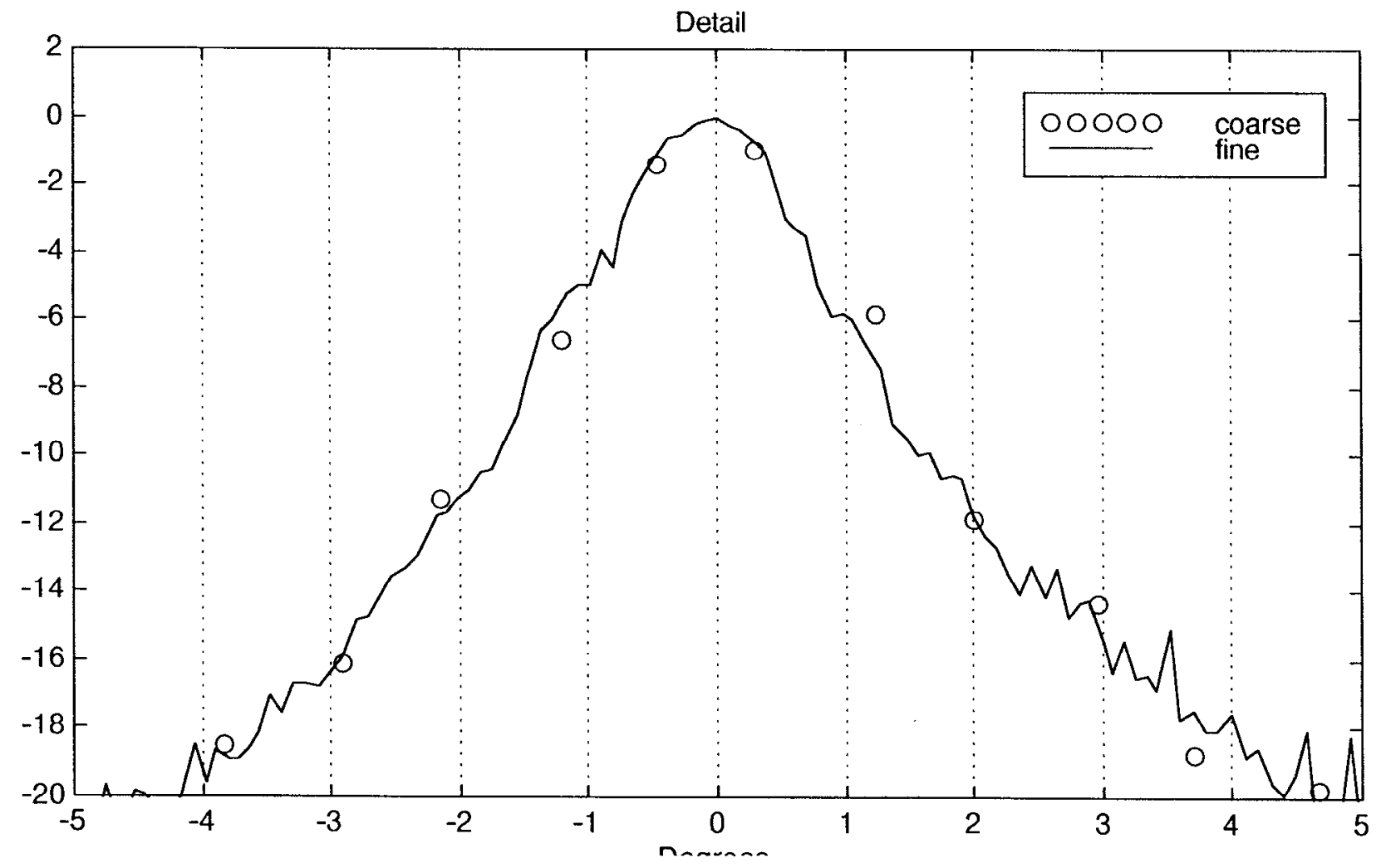




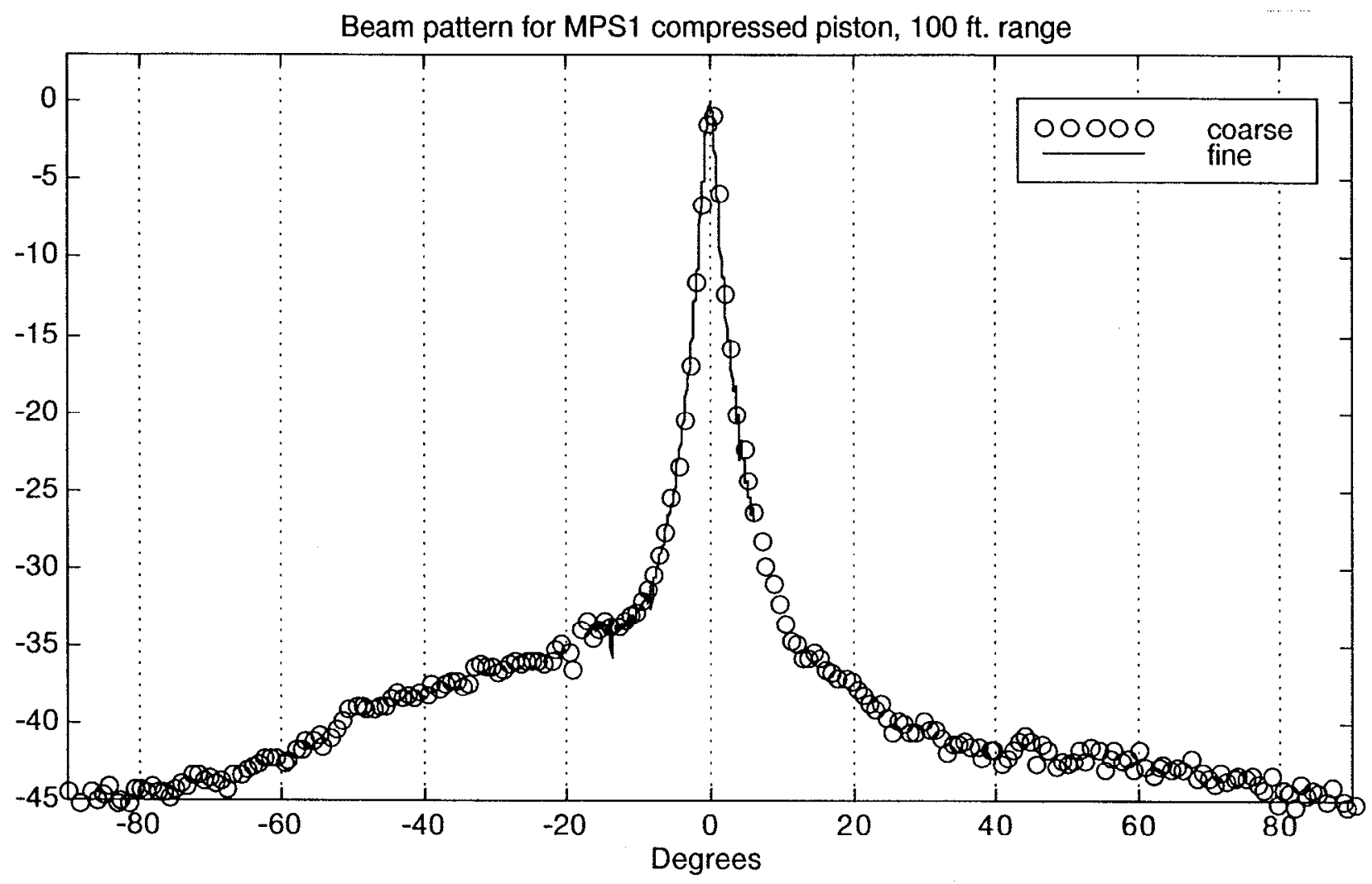

Figure E1-11. Beam pattern for MPS1 recompressed piston drive with no compensation at a 100 foot range. Above is -90 to +90 degrees, below is -5 to +5 degrees. Amplitudes are in $\mathrm{dB}$.

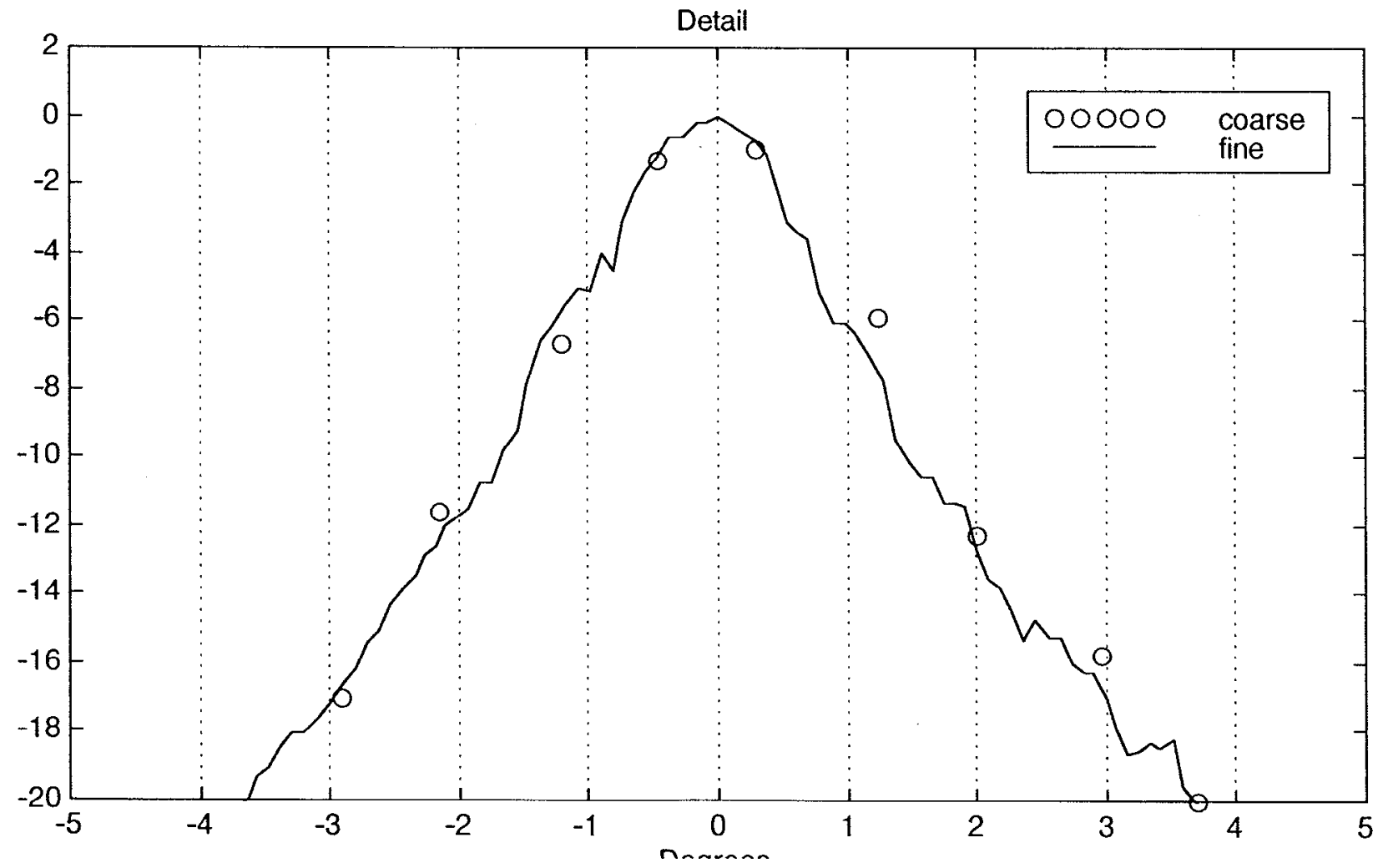




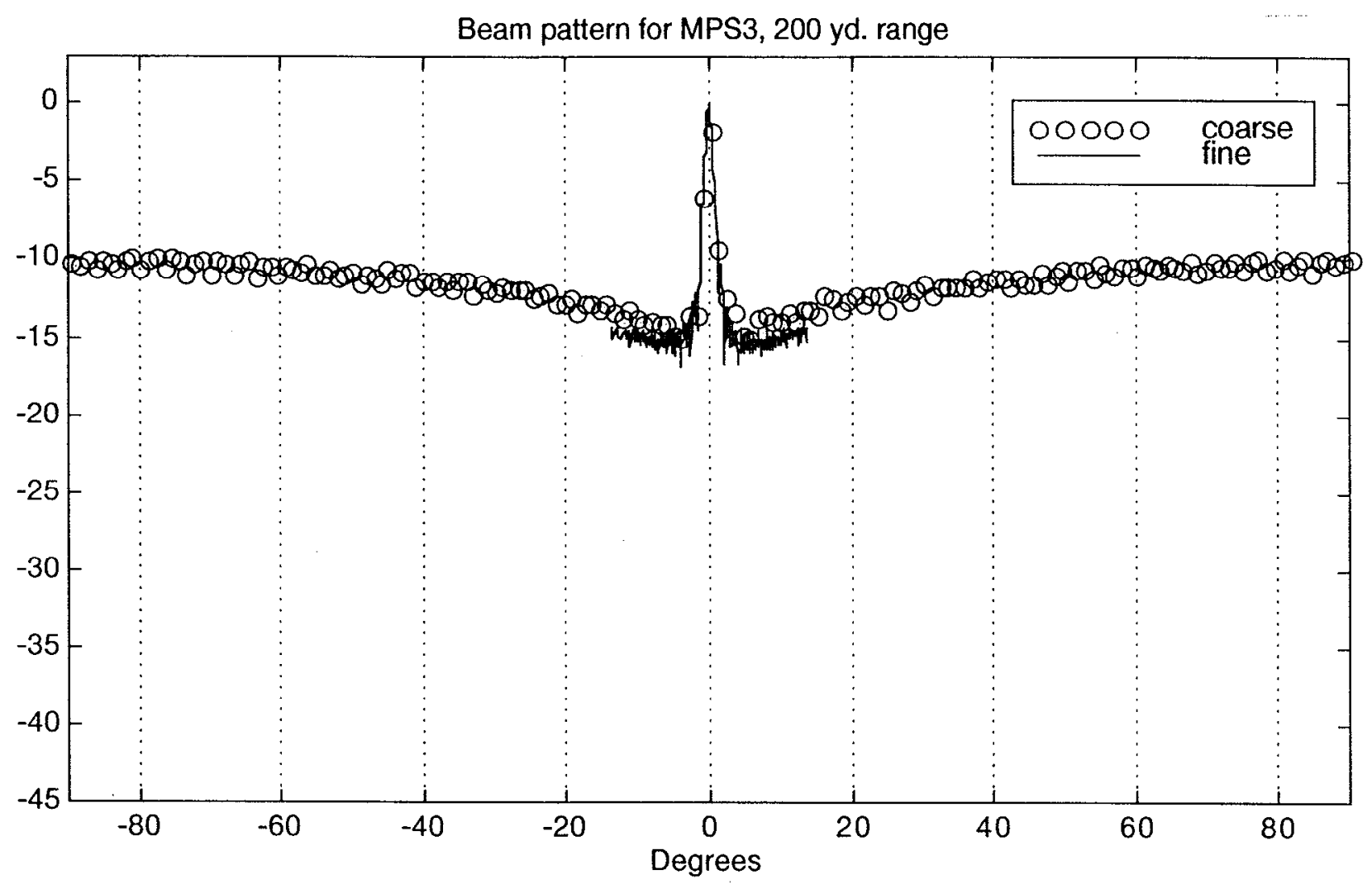

Figure E1-12. Beam pattern for MPS3 with no compensation at a 600 foot range. Above is -90 to +90 degrees, below is -5 to +5 degrees. Amplitudes are in $\mathrm{dB}$.

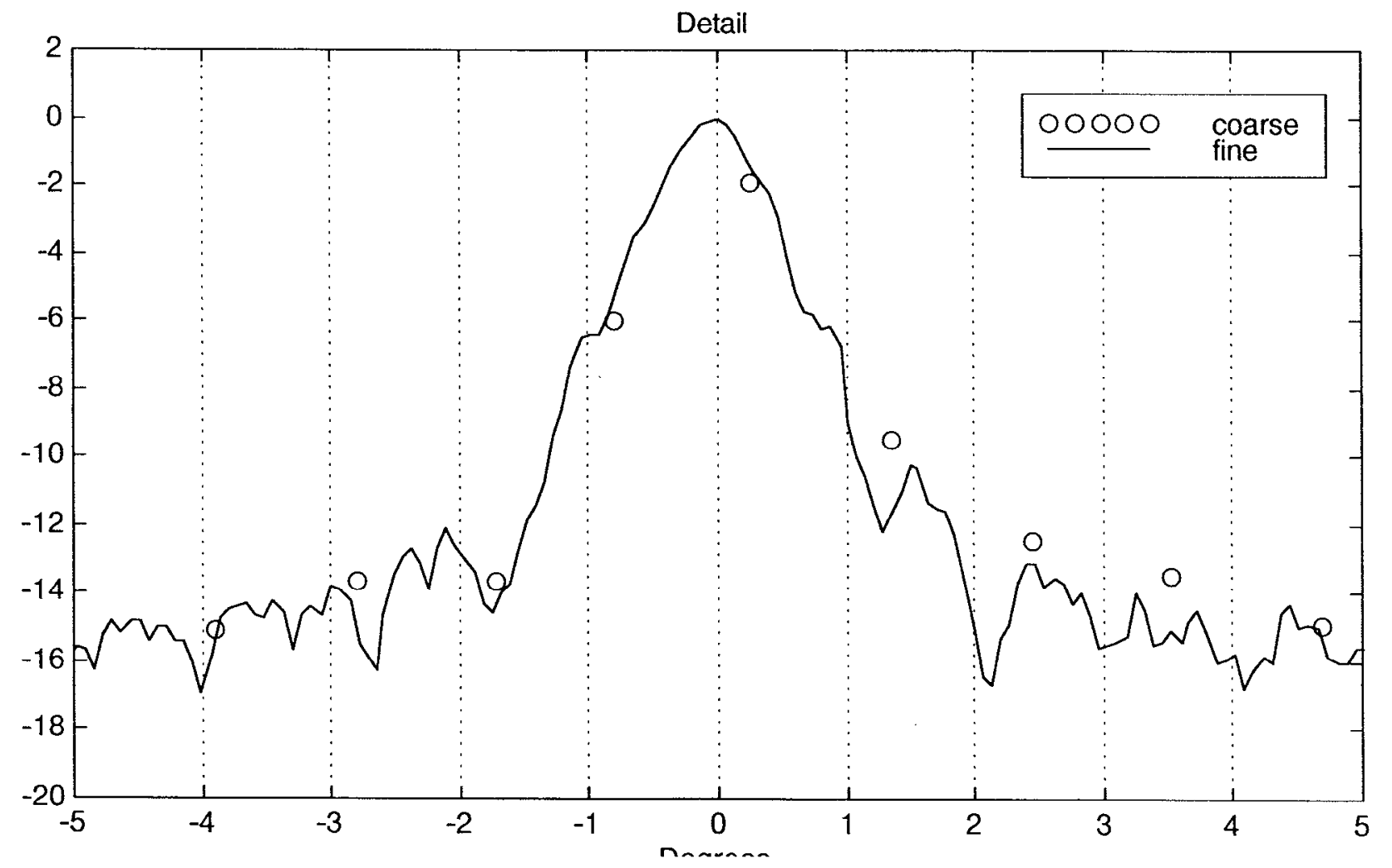


Beam pattern for MPS3 piston, 200 yd. range

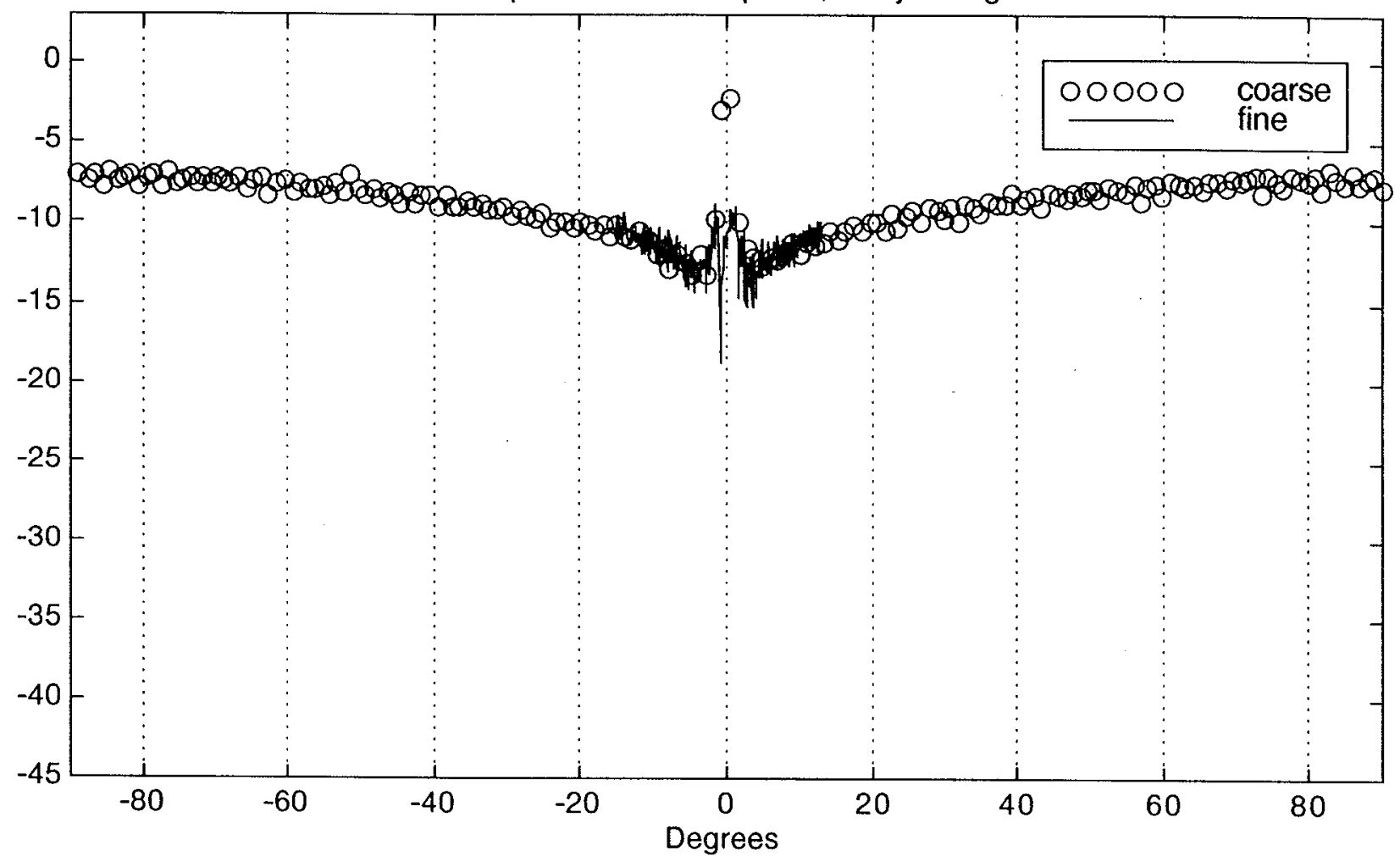

Figure E1-13. Beam pattern for MPS3 piston drive with no compensation at a 600 foot range. Above is -90 to +90 degrees, below is -5 to +5 degrees. Amplitudes are in $\mathrm{dB}$. Fine Scan data faulty due to environment.

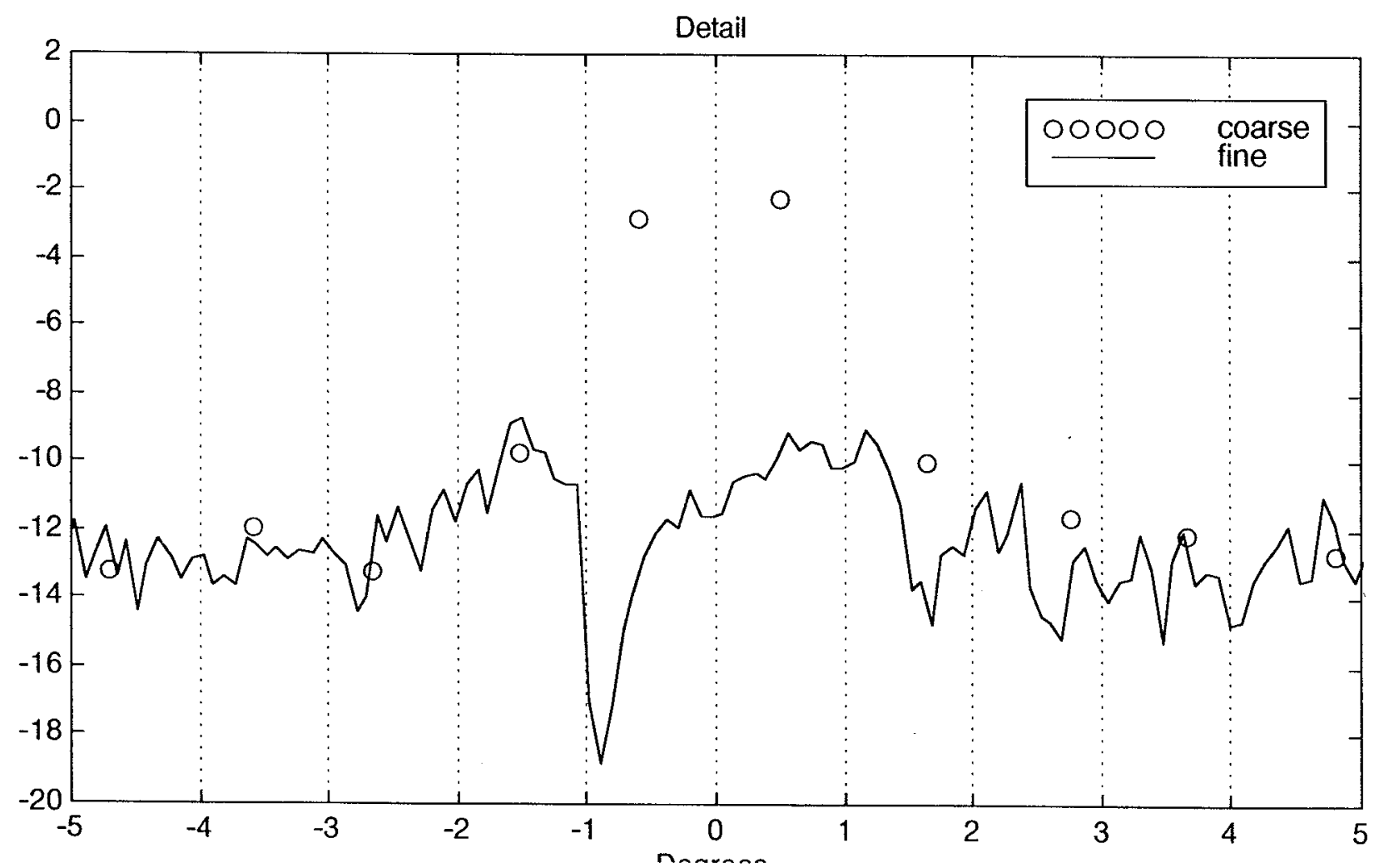


Beam pattern for MPS3 stretched, $200 \mathrm{yd}$. range

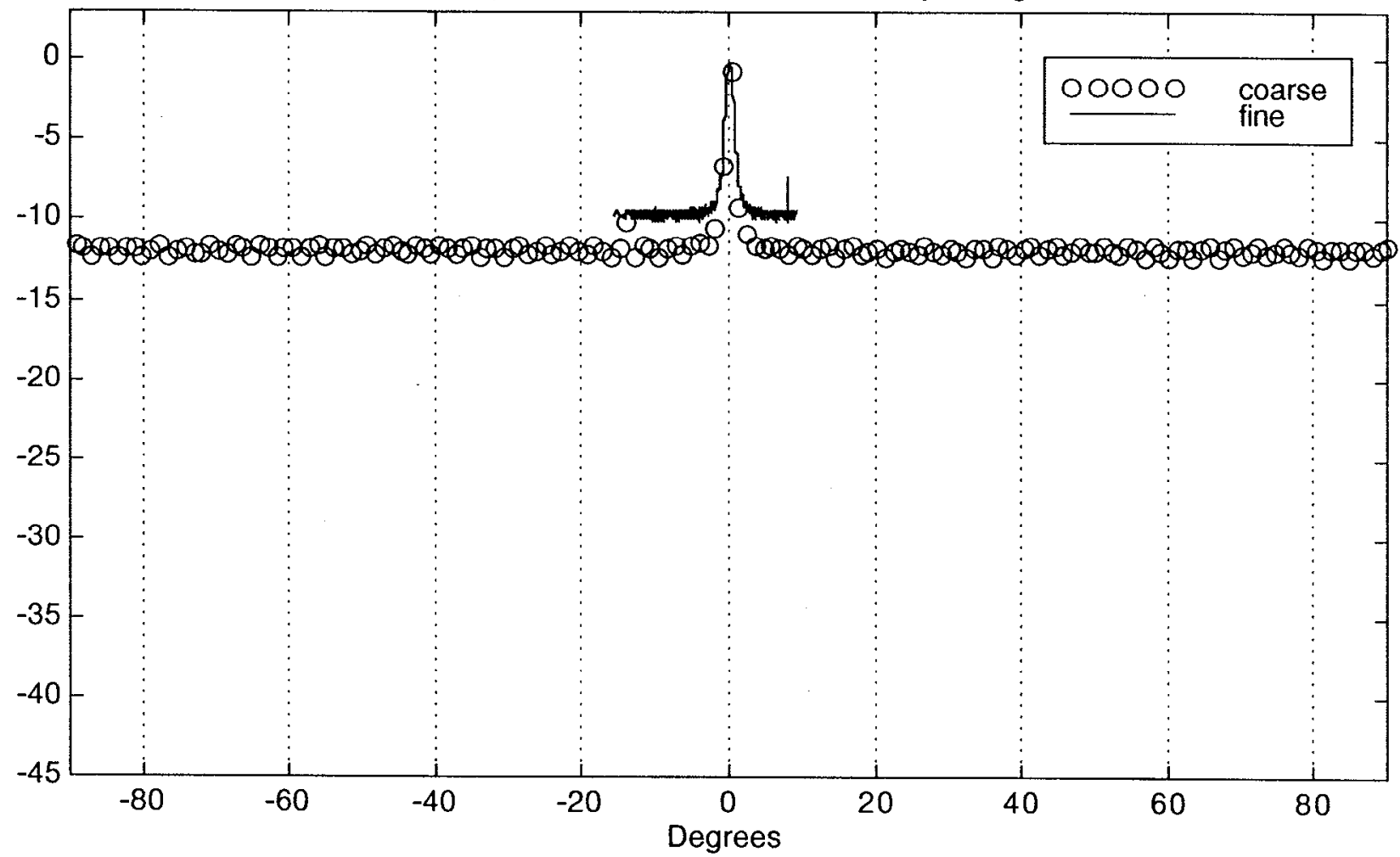

Figure E1-14. Beam pattern for MPS3 stretched with no compensation at a 600 foot range. Above is -90 to +90 degrees, below is -5 to +5 degrees.

Amplitudes are in $\mathrm{dB}$.

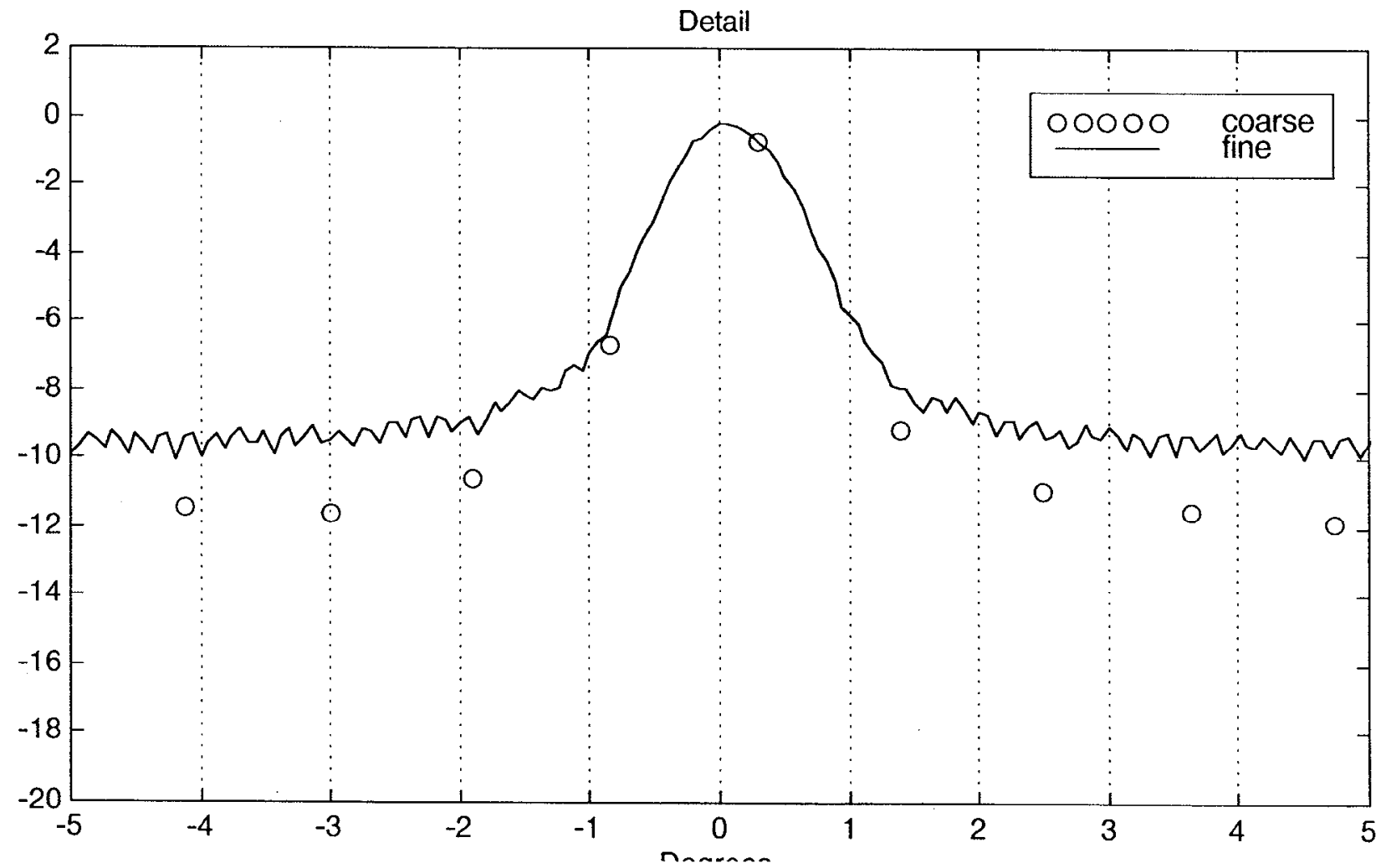


Beam pattern for MPS3 compressed, 200 yd. range

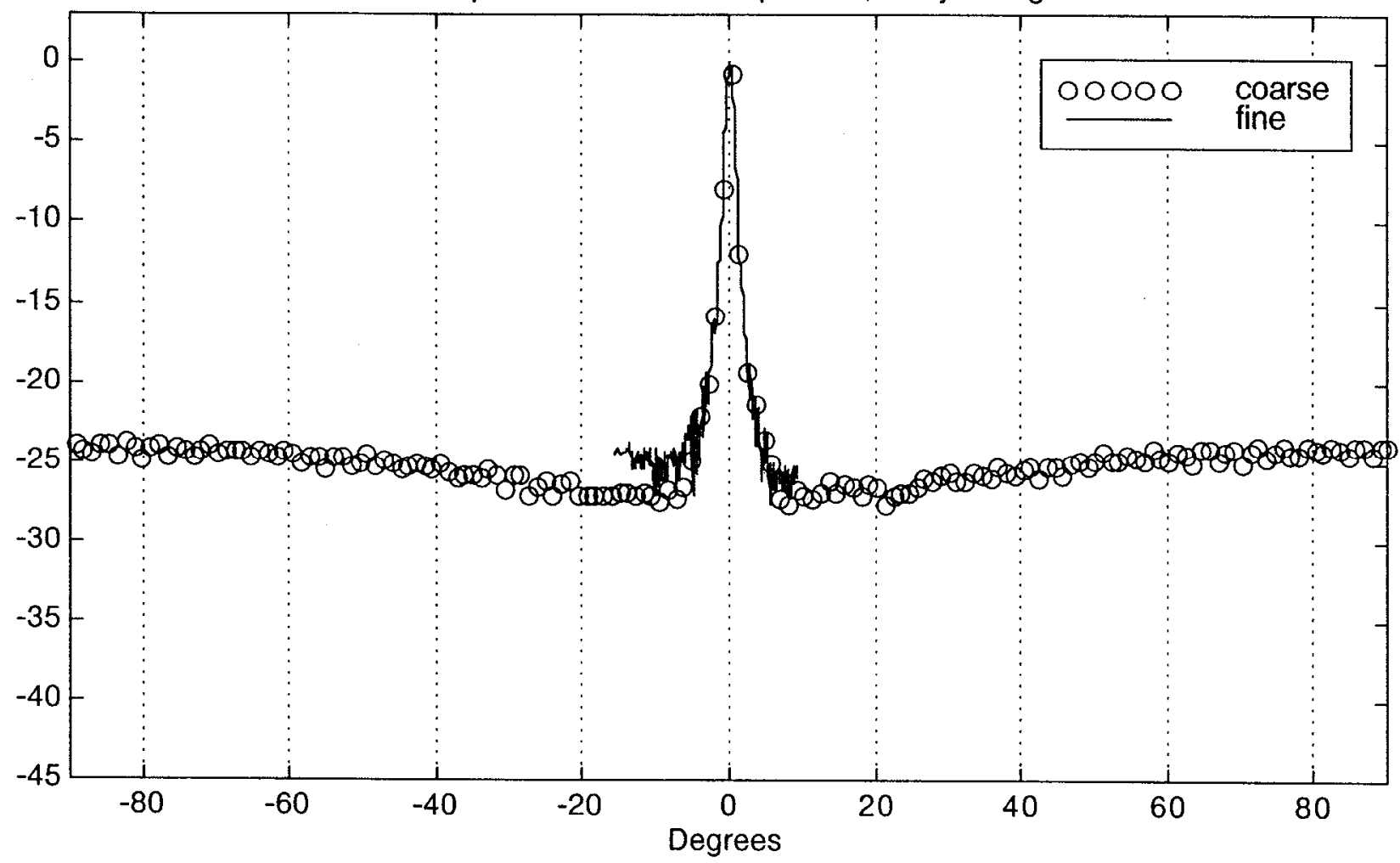

Figure E1-15. Beam pattern for MPS3 stretched and recompressed with no compensation at a 600 foot range. Above is -90 to +90 degrees, below is -5 to +5 degrees. Amplitudes are in $\mathrm{dB}$.

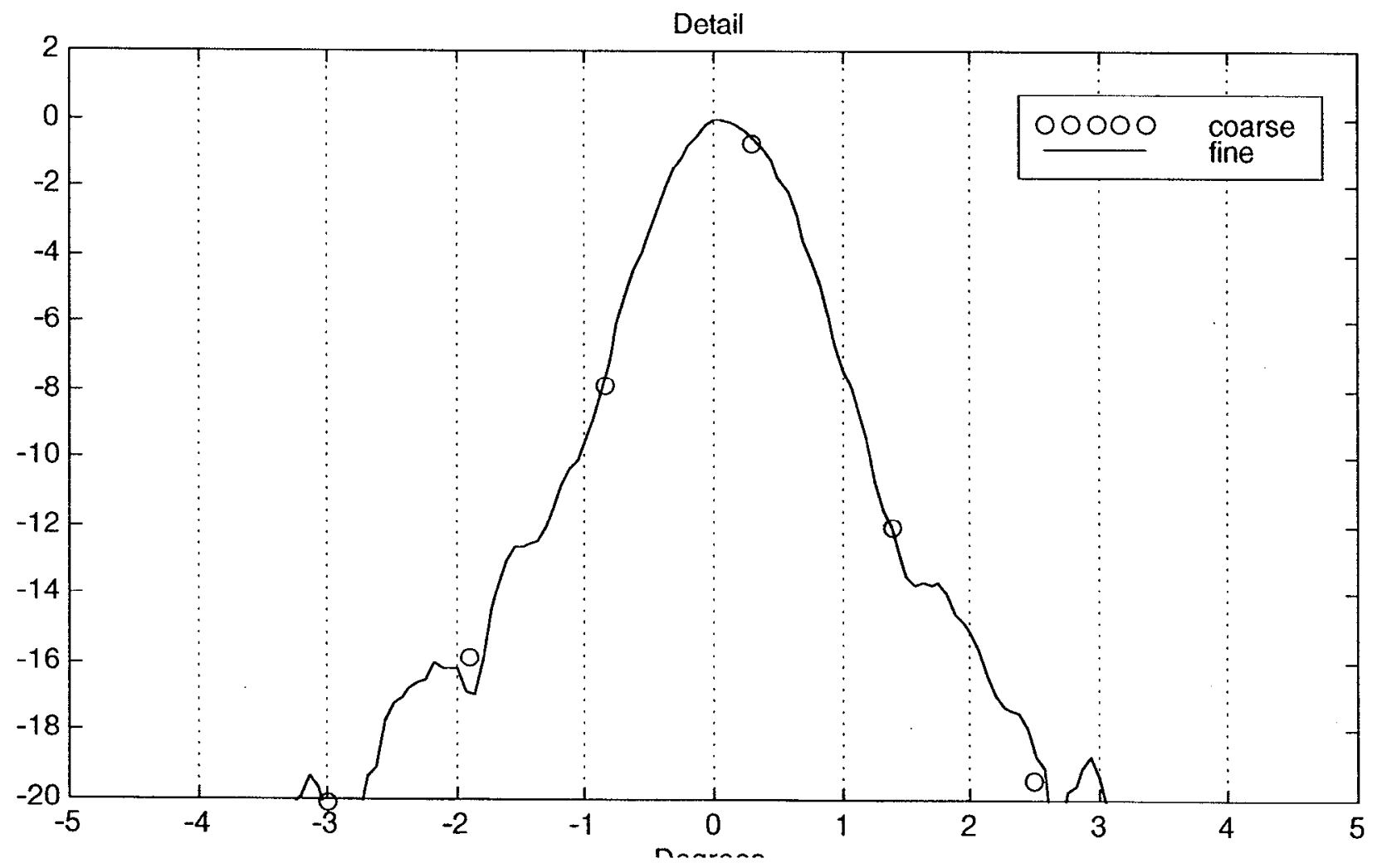


Beam pattern for MPS3 stretched piston, 200 yd. range

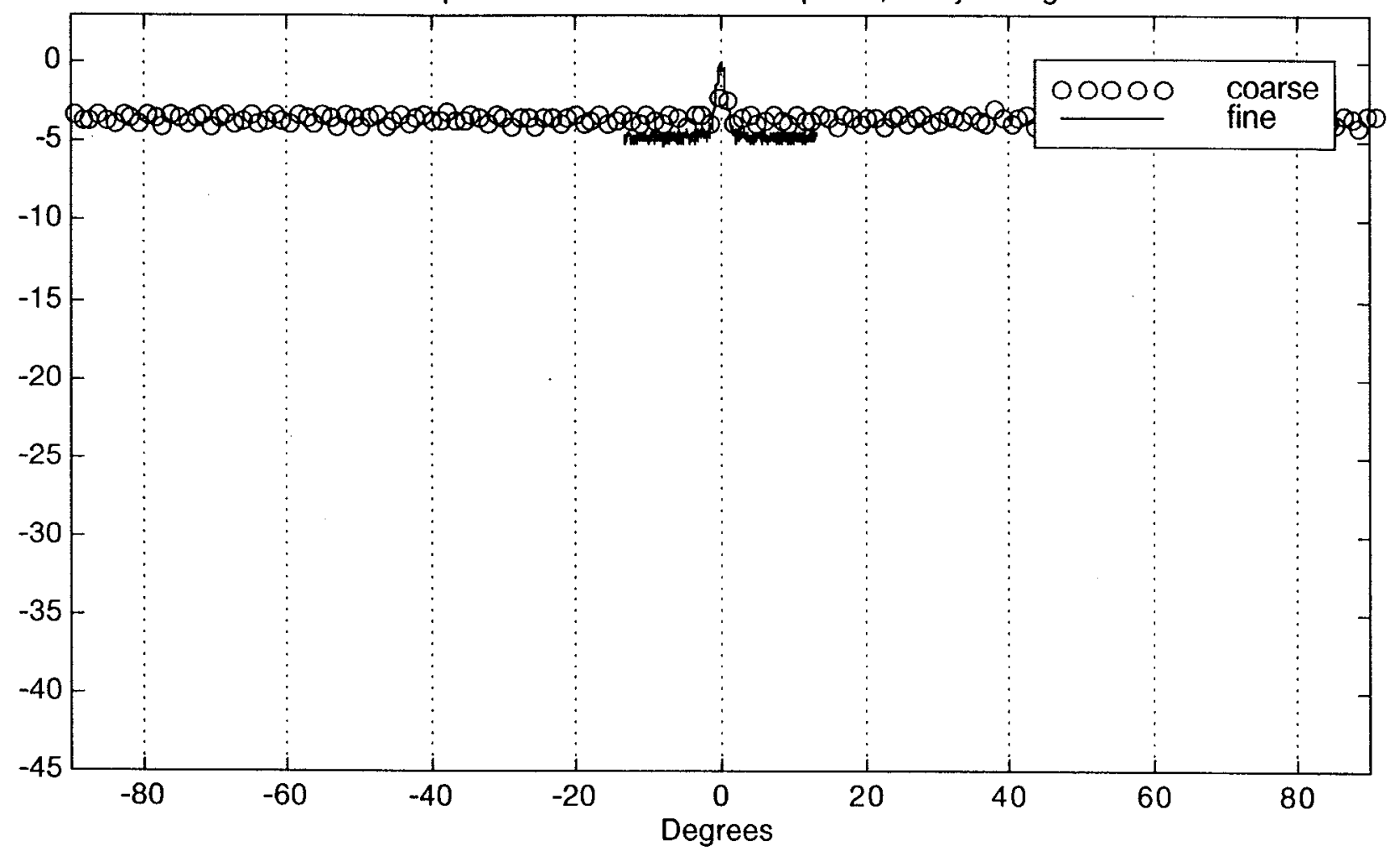

Figure E1-16. Beam pattern for MPS3 piston drive stretched with no compensation at a 600 foot range. Above is -90 to +90 degrees, below is -5 to +5 degrees. Amplitudes are in $\mathrm{dB}$.

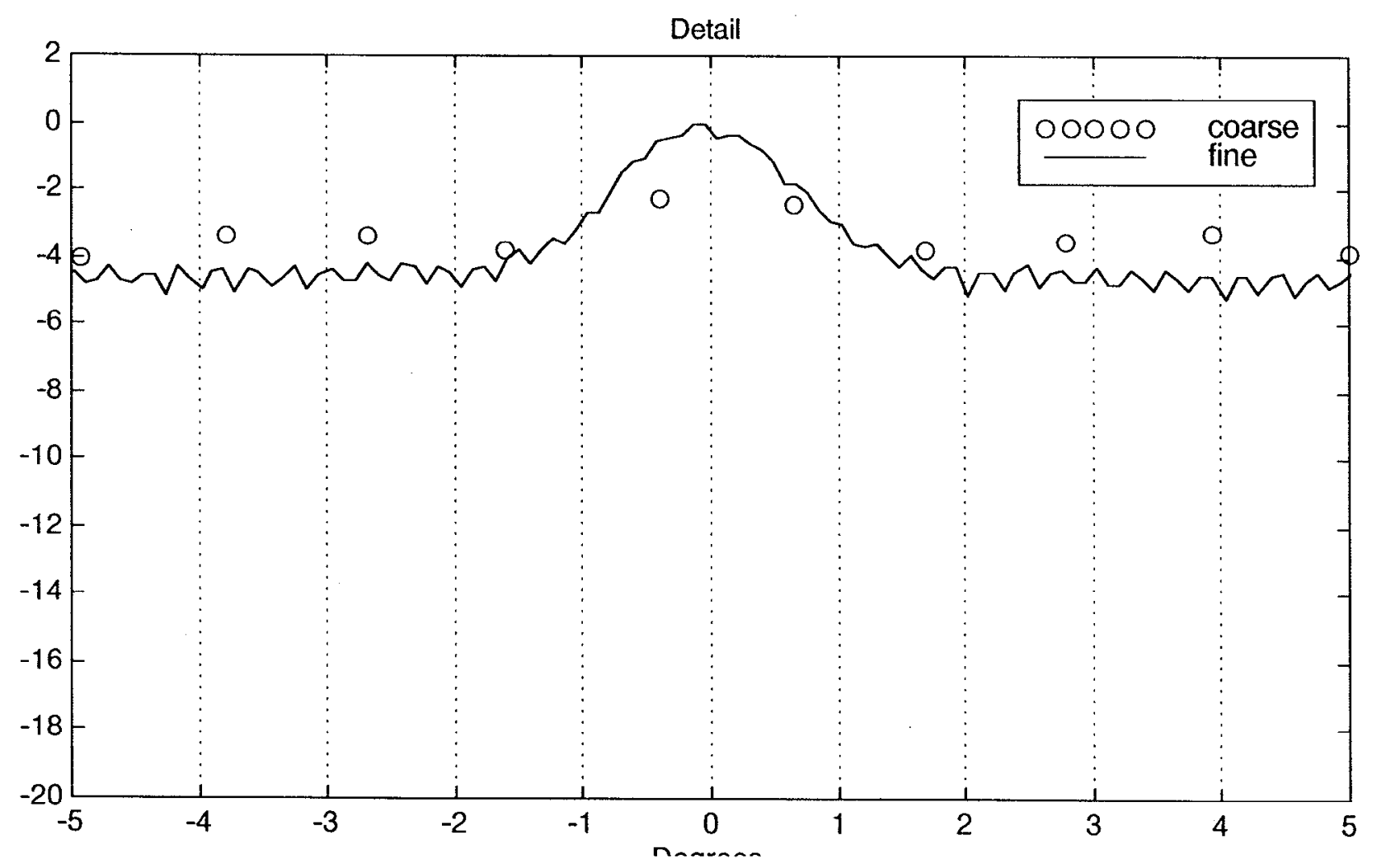


Beam pattern for MPS3 compressed piston, 200 yd. range

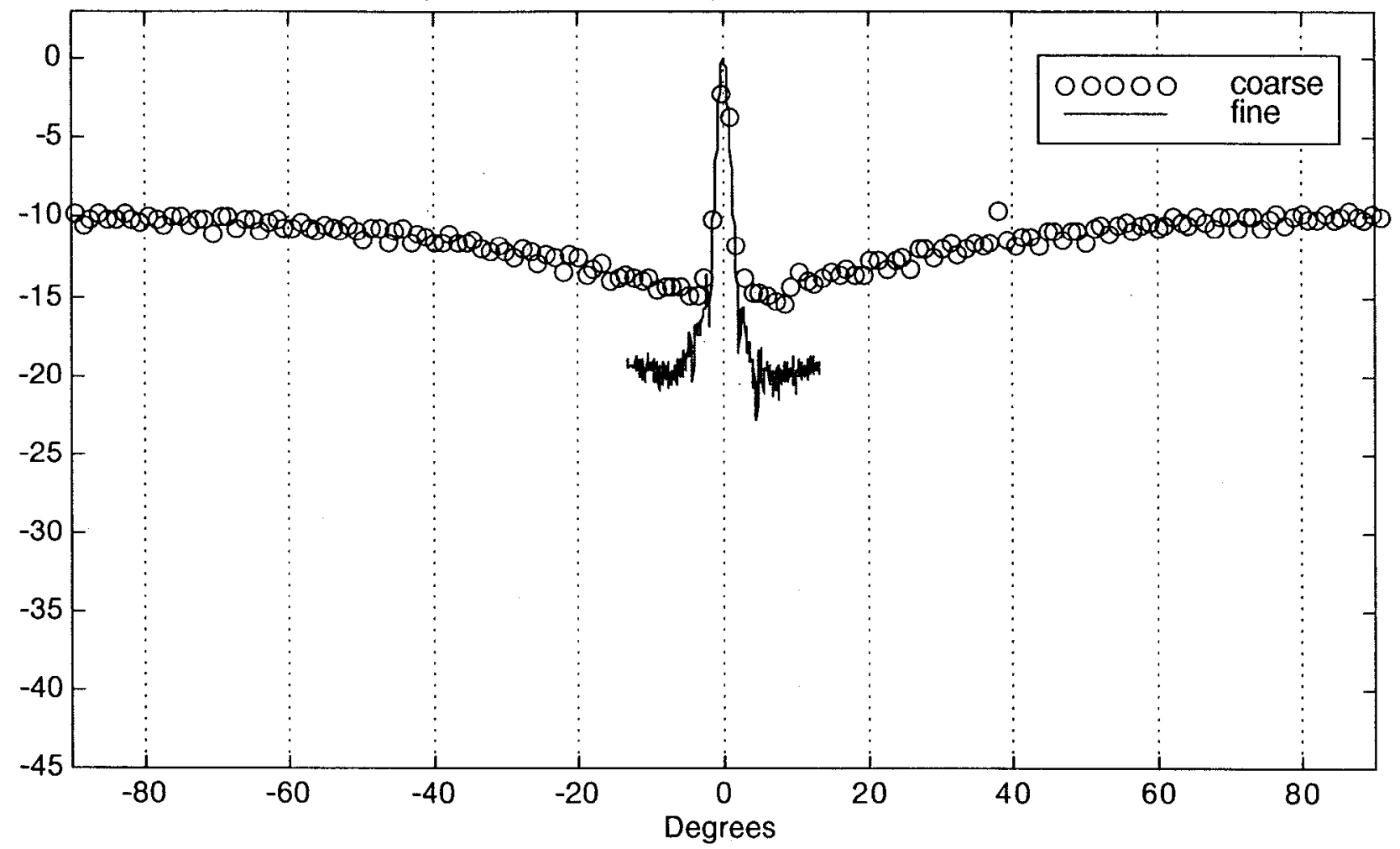

Figure E1-17. Beam pattern for MPS3 piston drive stretched and recompressed with no compensation at a 600 foot range. Above is -90 to +90 degrees, below is -5 to +5 degrees. Amplitudes are in $\mathrm{dB}$.

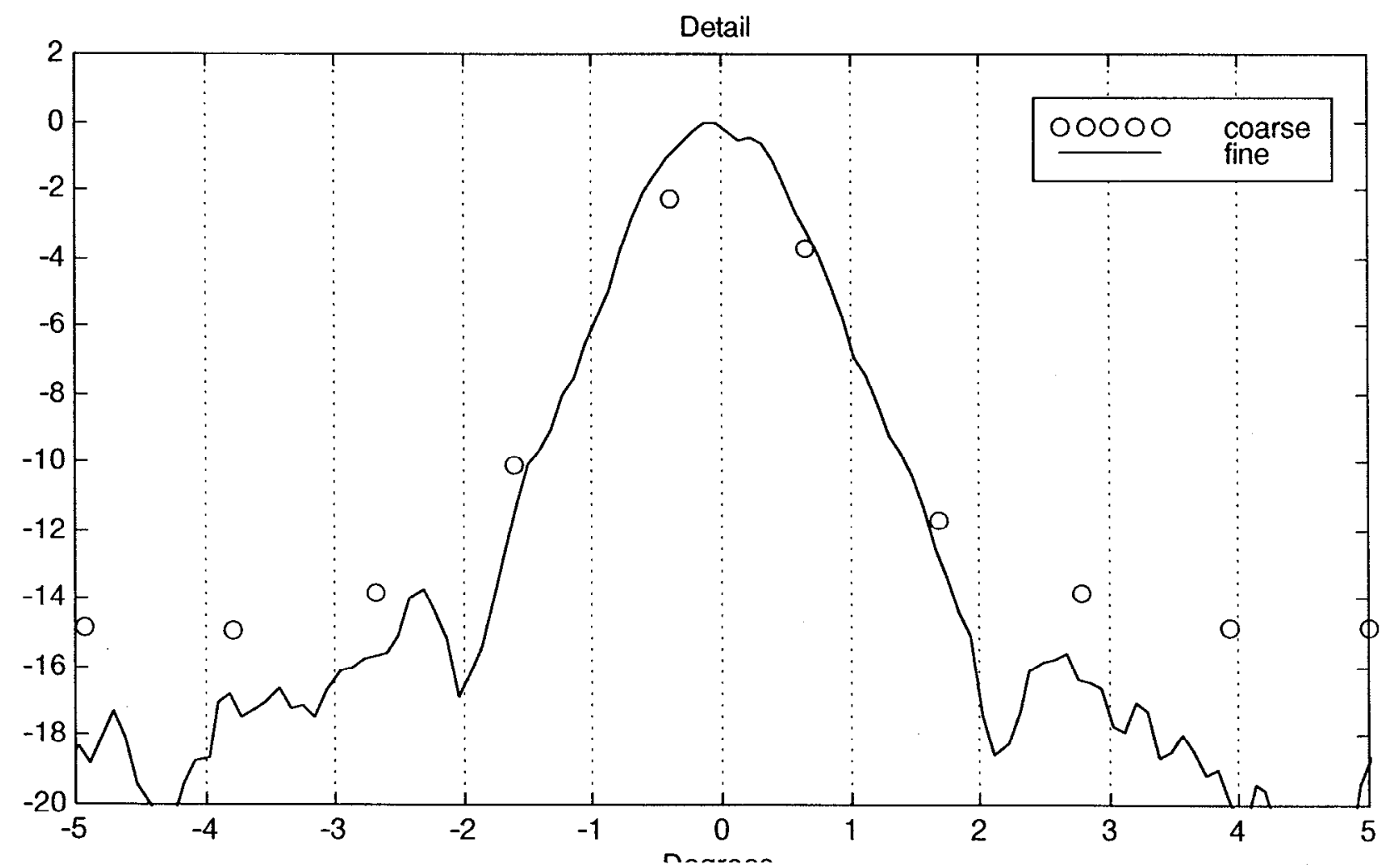


Beam pattern for SG1 NC, $100 \mathrm{ft}$. range

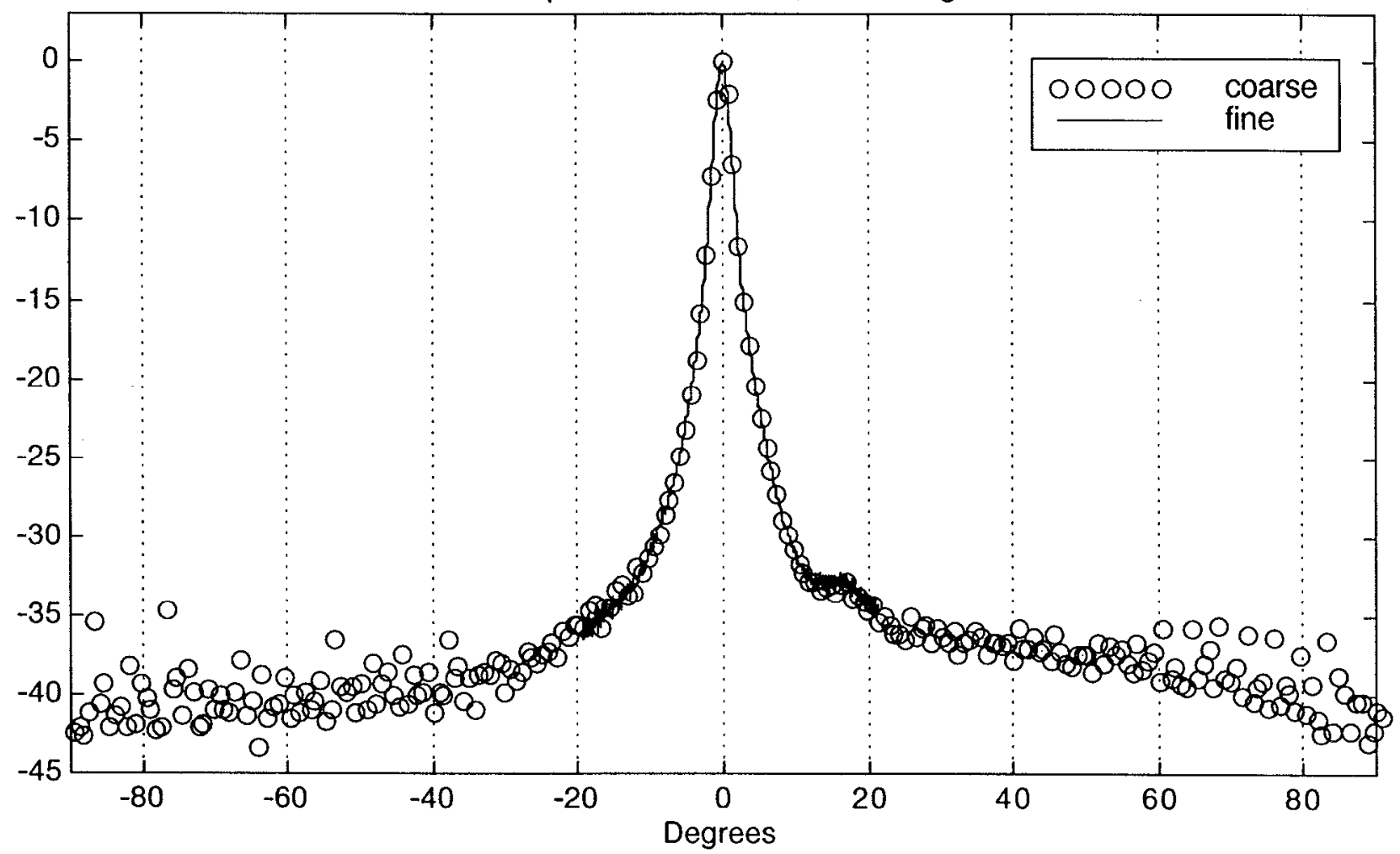

Figure E1-18. Beam pattern for SG1 with no compensation at a 100 foot range. Above is -90 to +90 degrees, below is -5 to +5 degrees. Amplitudes are in $\mathrm{dB}$.

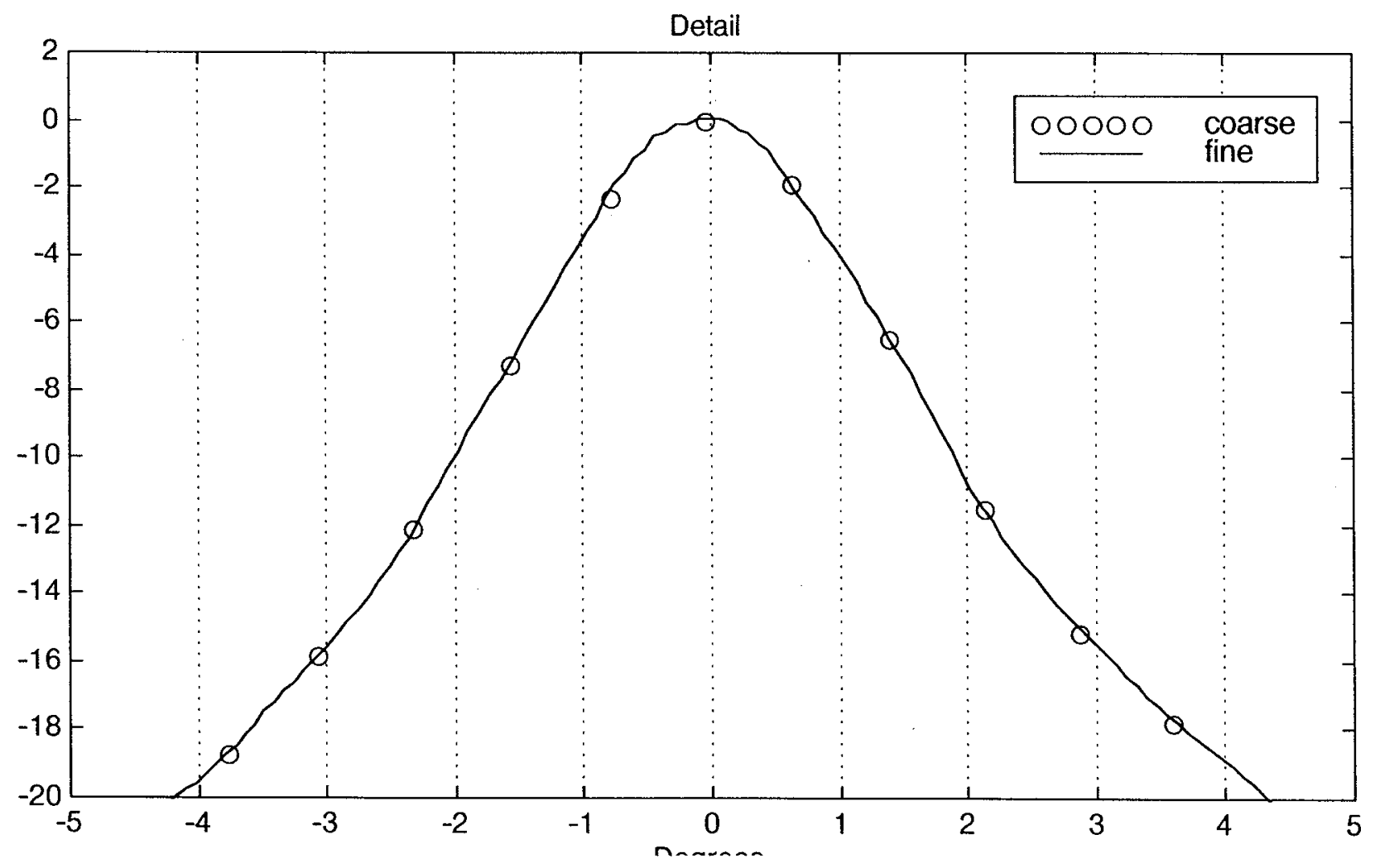


Beam pattern for SG1 2D, $100 \mathrm{ft}$. range

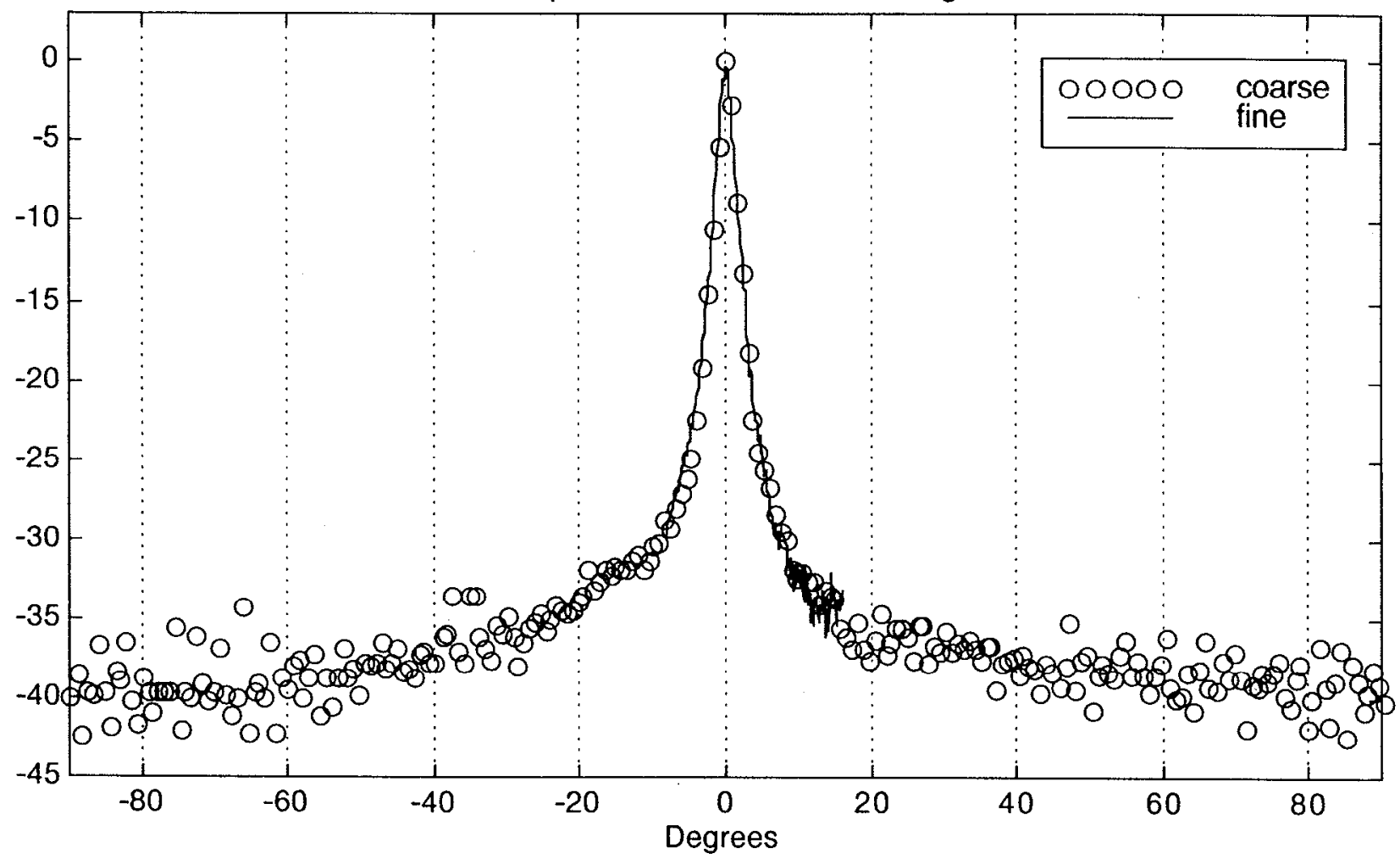

Figure E1-19. Beam pattern for SG1 with $2^{\text {nd }}$ derivative compensation at a 100 foot range. Above is -90 to +90 degrees, below is -5 to +5 degrees. Amplitudes are in $\mathrm{dB}$.

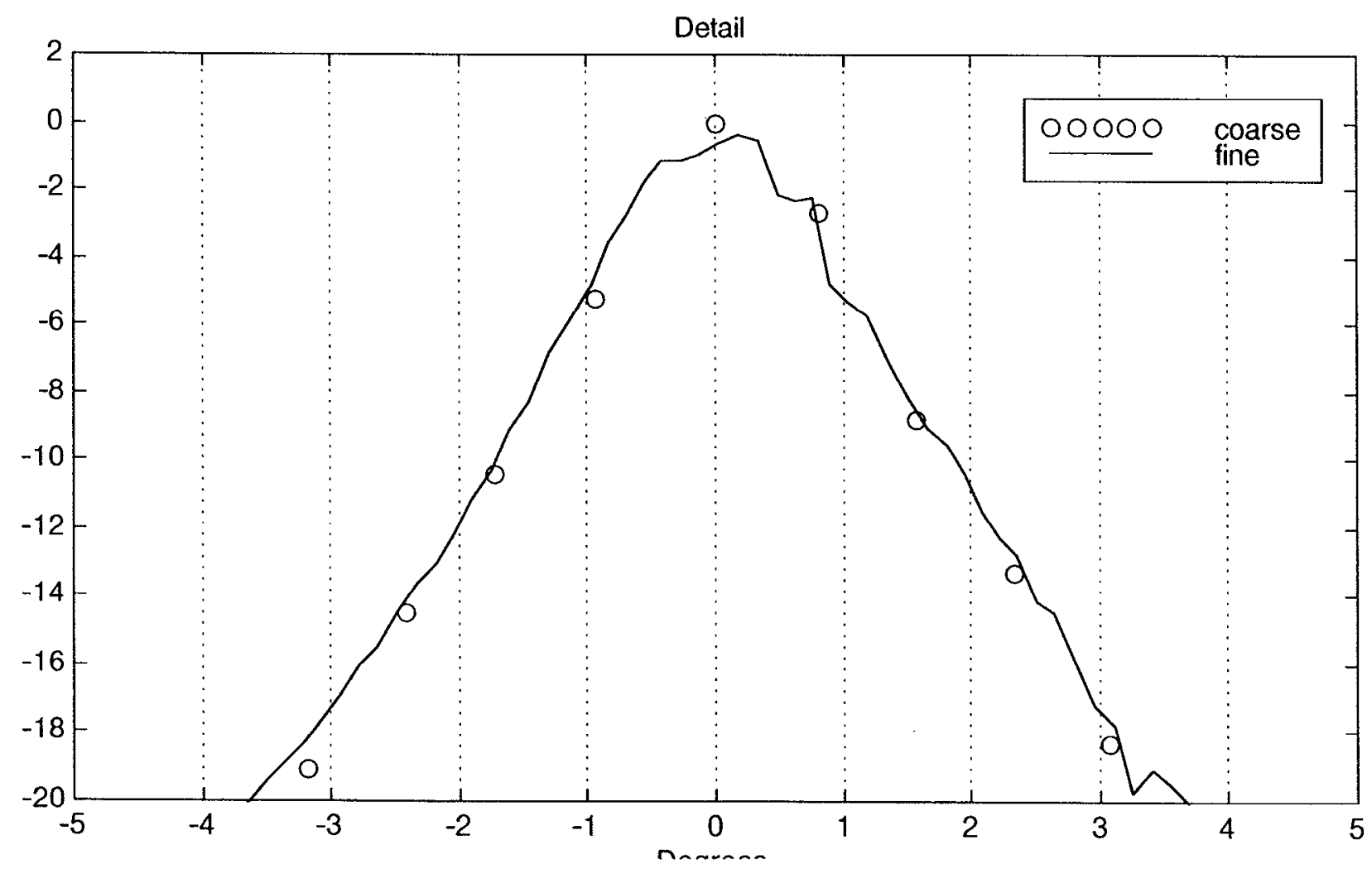


Beam pattern for SG1 2D piston, $100 \mathrm{ft}$. range

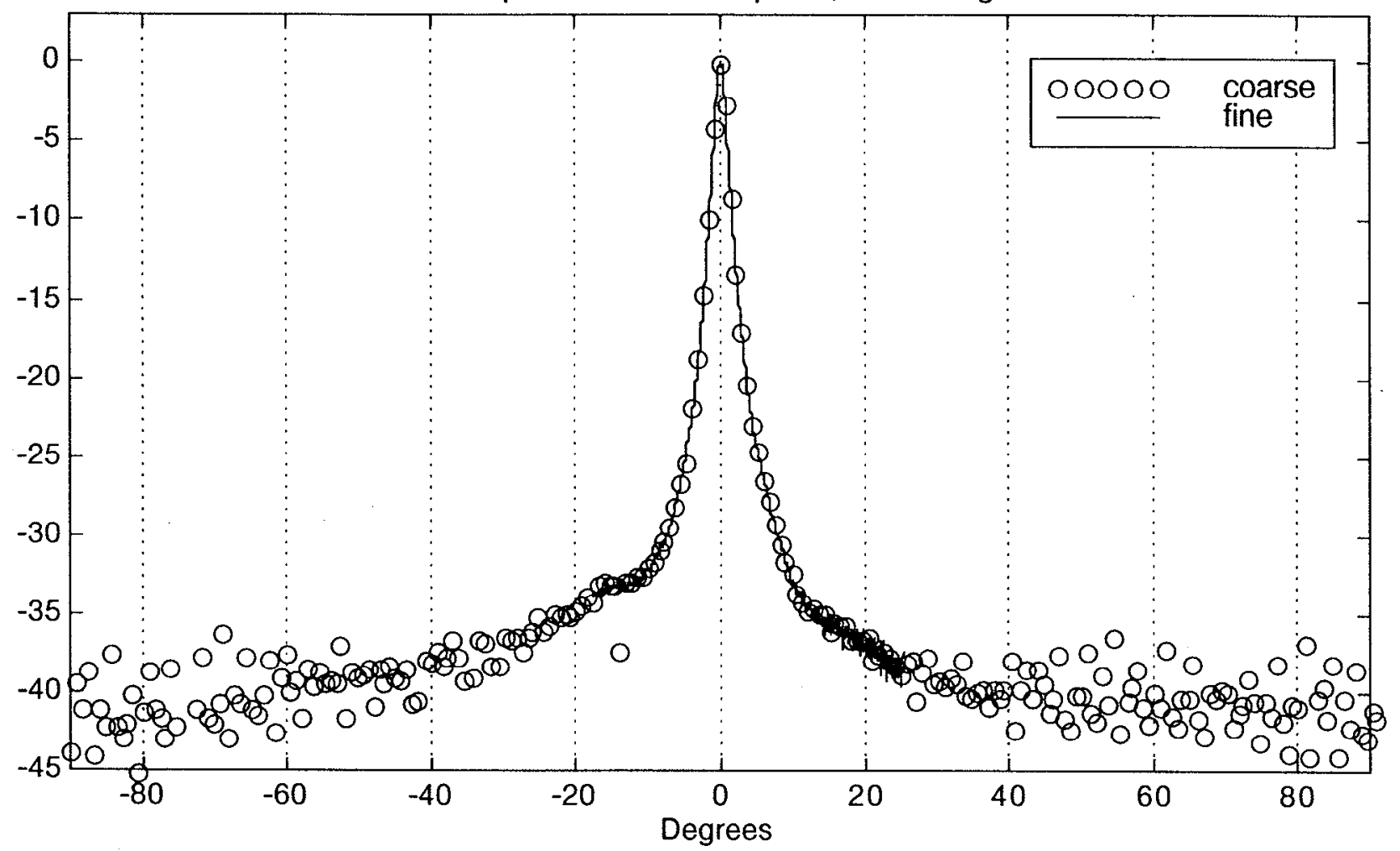

Figure E1-20. Beam pattern for SG1 piston drive with $2^{\text {nd }}$ derivative compensation at a 100 foot range. Above is -90 to +90 degrees, below is -5 to +5 degrees. Amplitudes are in $\mathrm{dB}$.

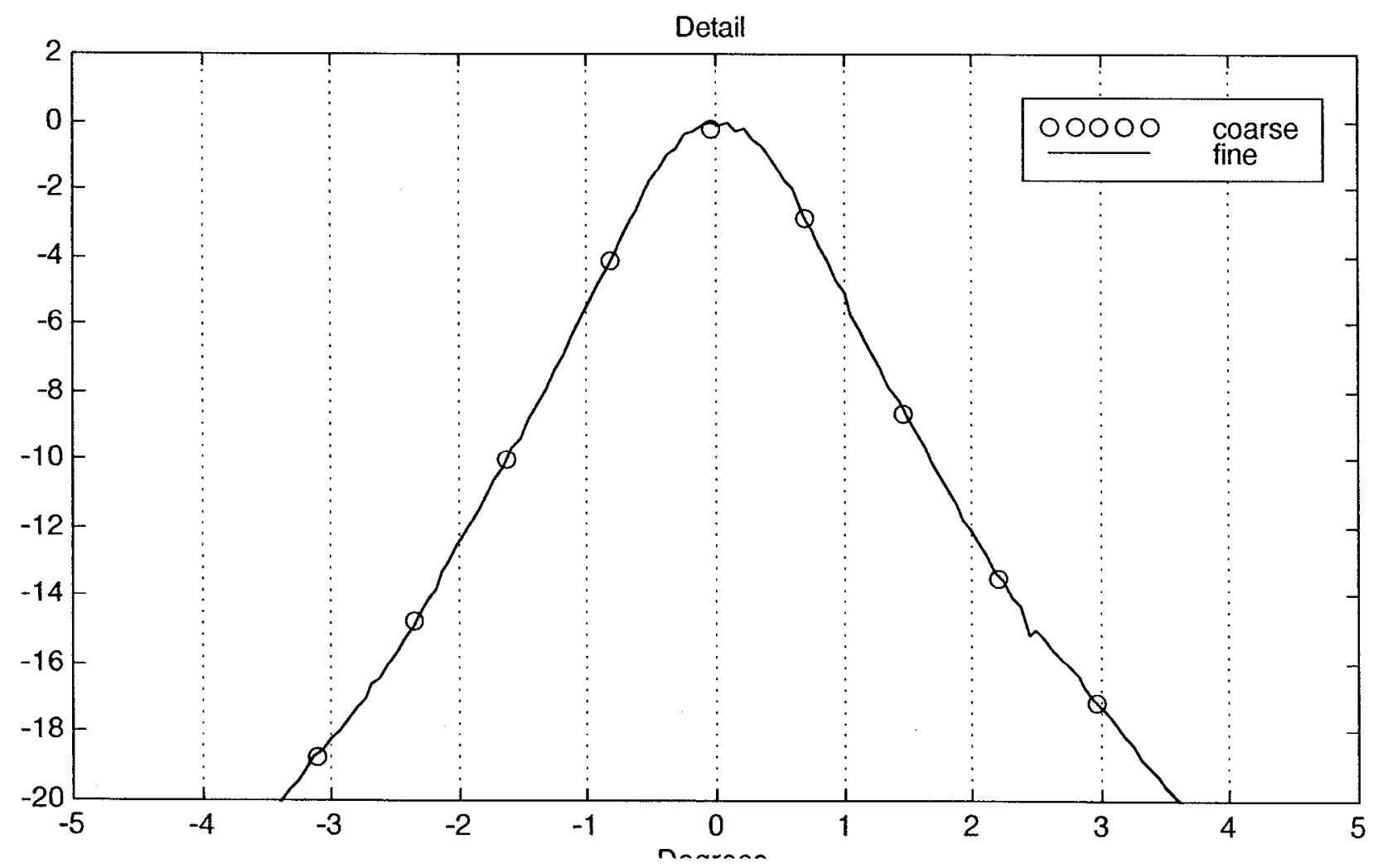




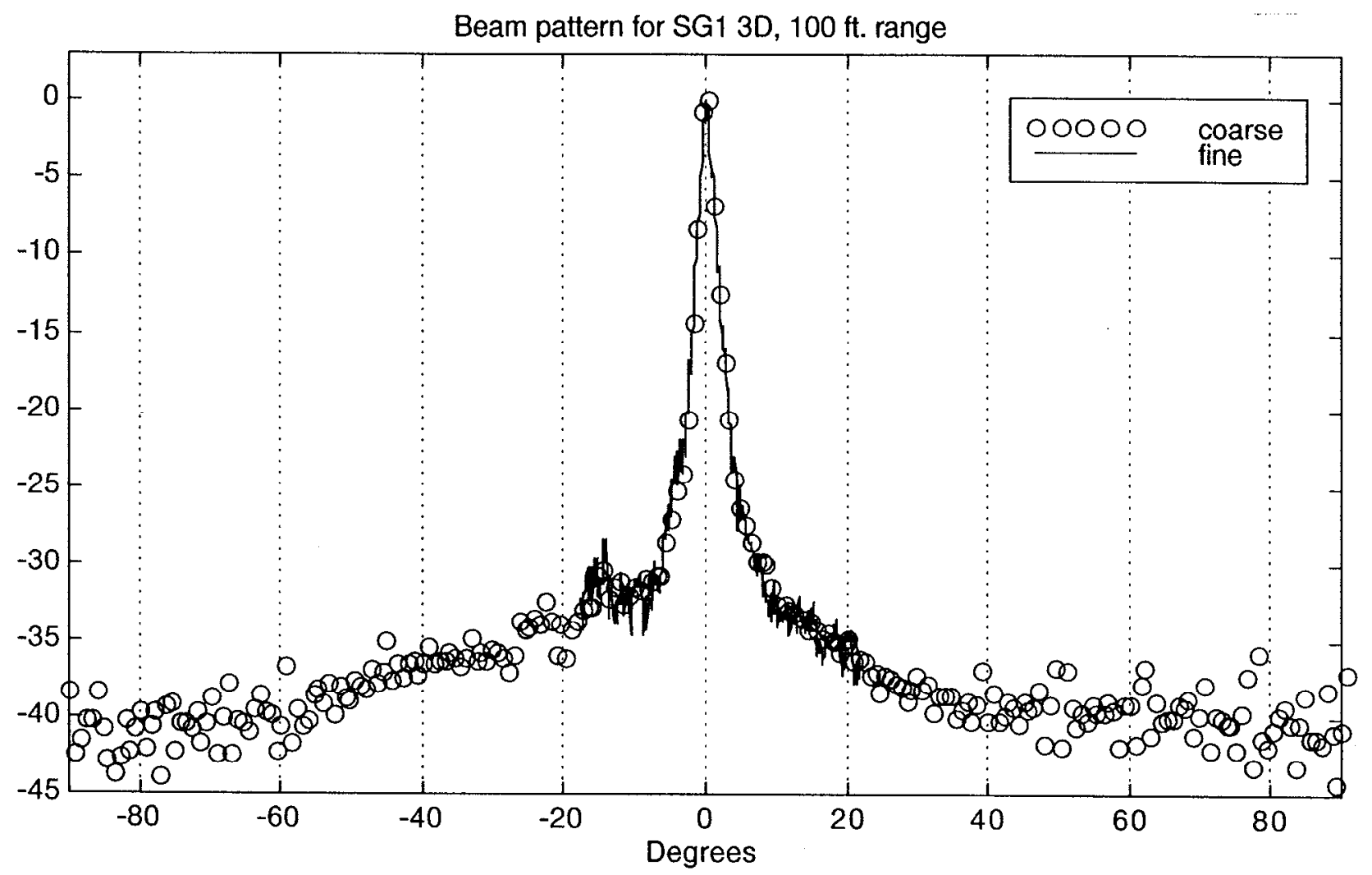

Figure E1-21. Beam pattern for SG1 with 3rd derivative compensation at a 100 foot range. Above is -90 to +90 degrees, below is -5 to +5 degrees. Amplitudes are in $\mathrm{dB}$.

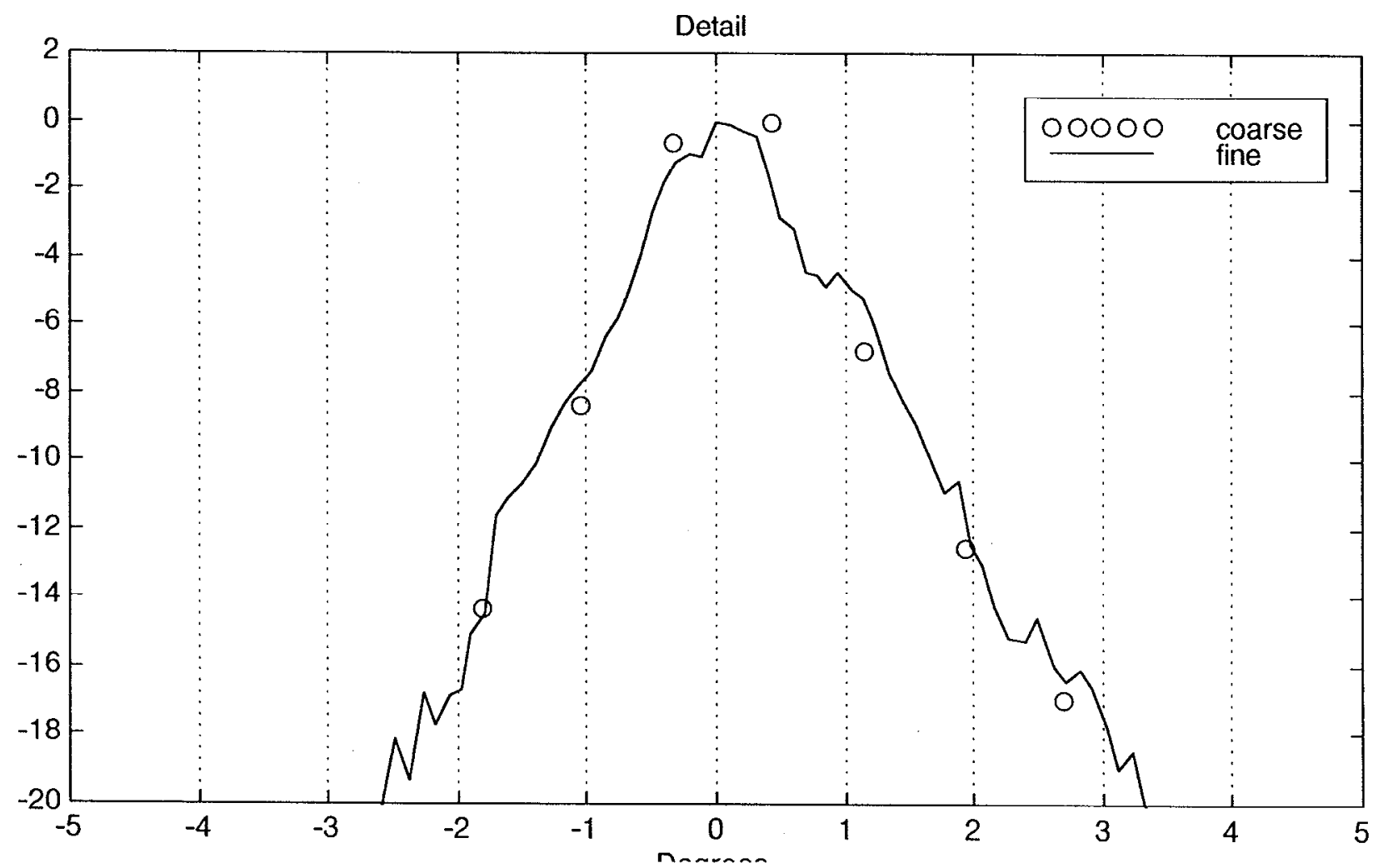


Beam pattern for SG1 3D piston, $100 \mathrm{ft}$. range

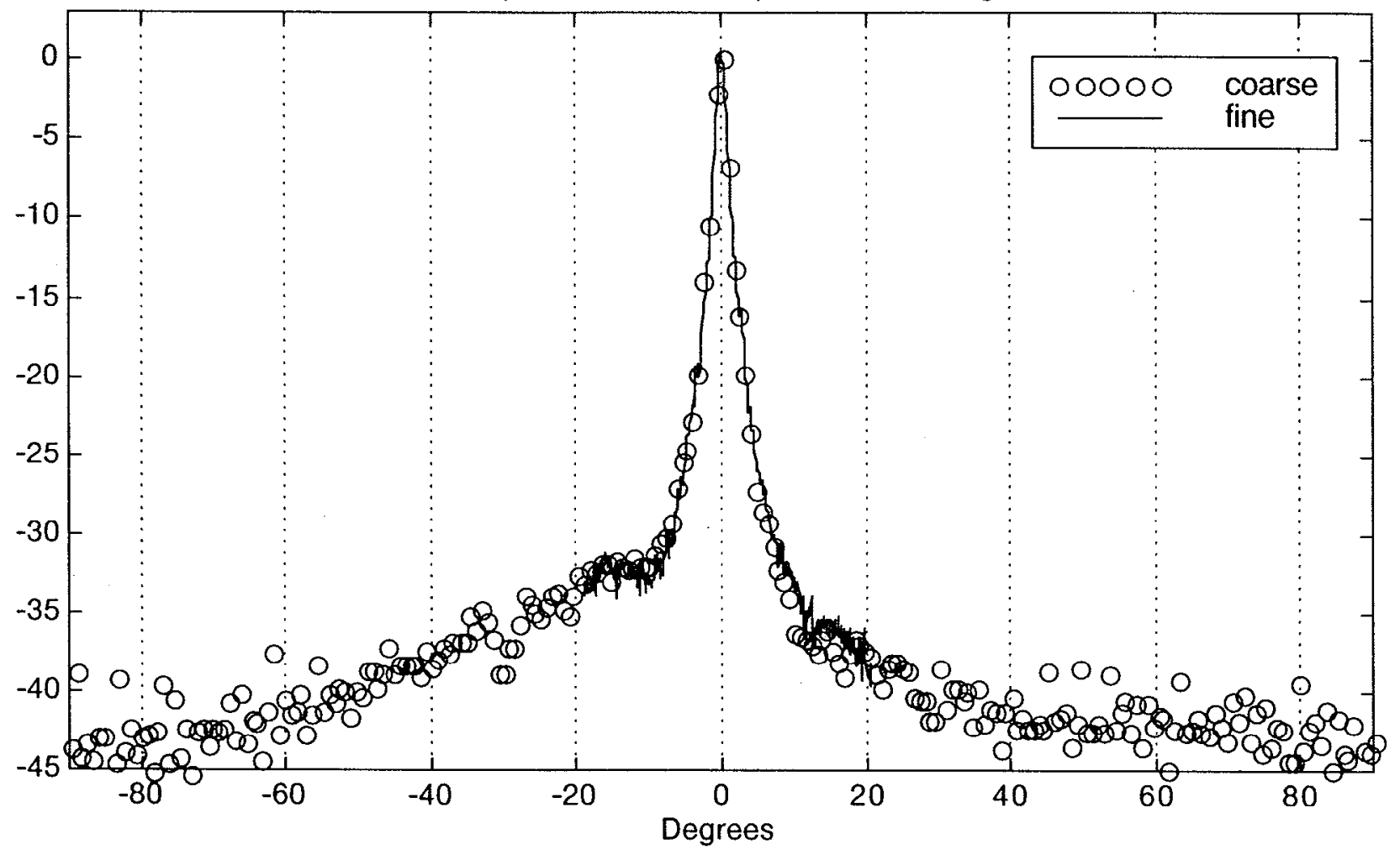

Figure E1-22. Beam pattern for SG1 piston drive with 3rd derivative compensation at a 100 foot range. Above is -90 to +90 degrees, below is -5 to +5 degrees. Amplitudes are in $\mathrm{dB}$.

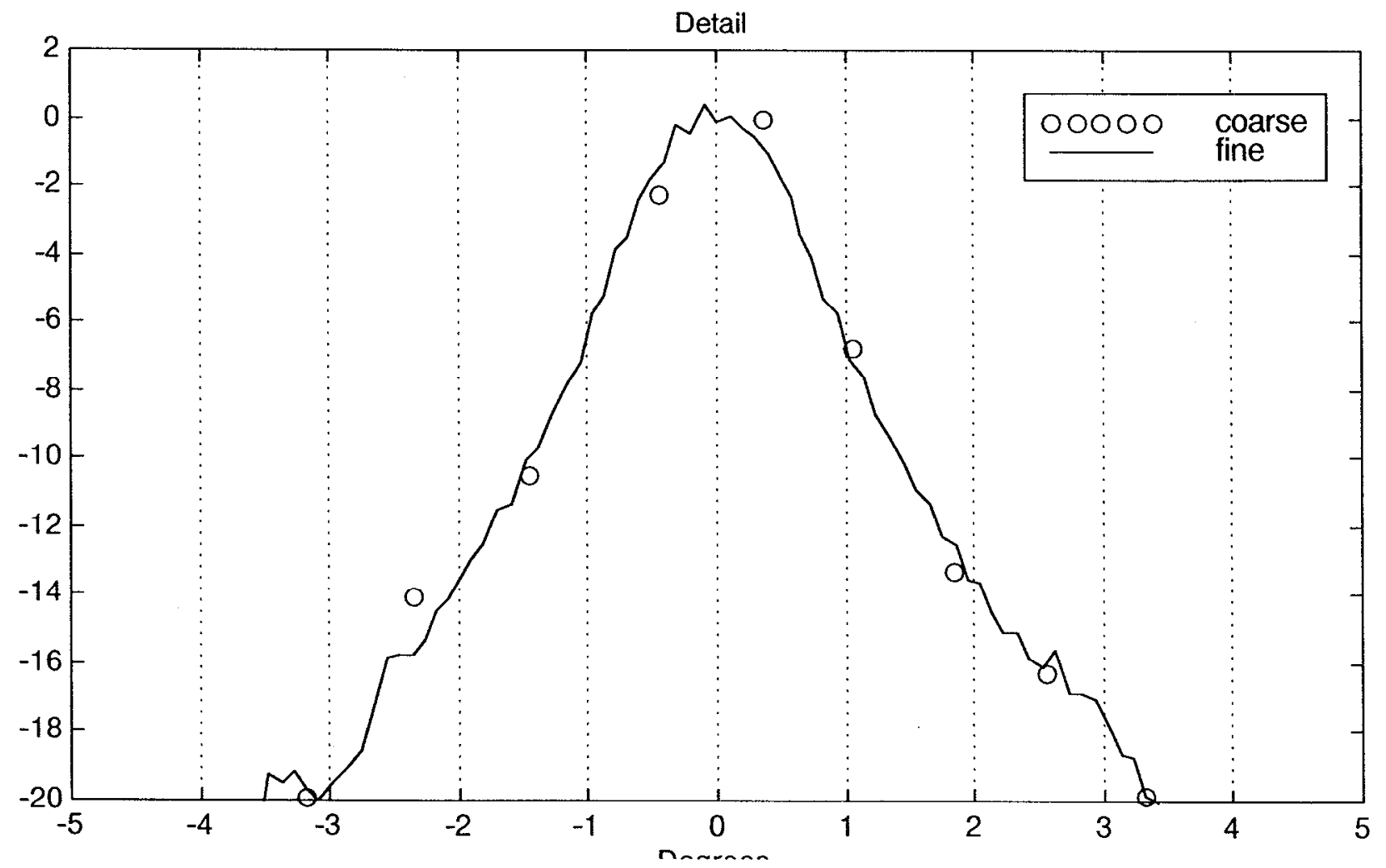


Beam pattern for SG1 NC, 200 yd. range

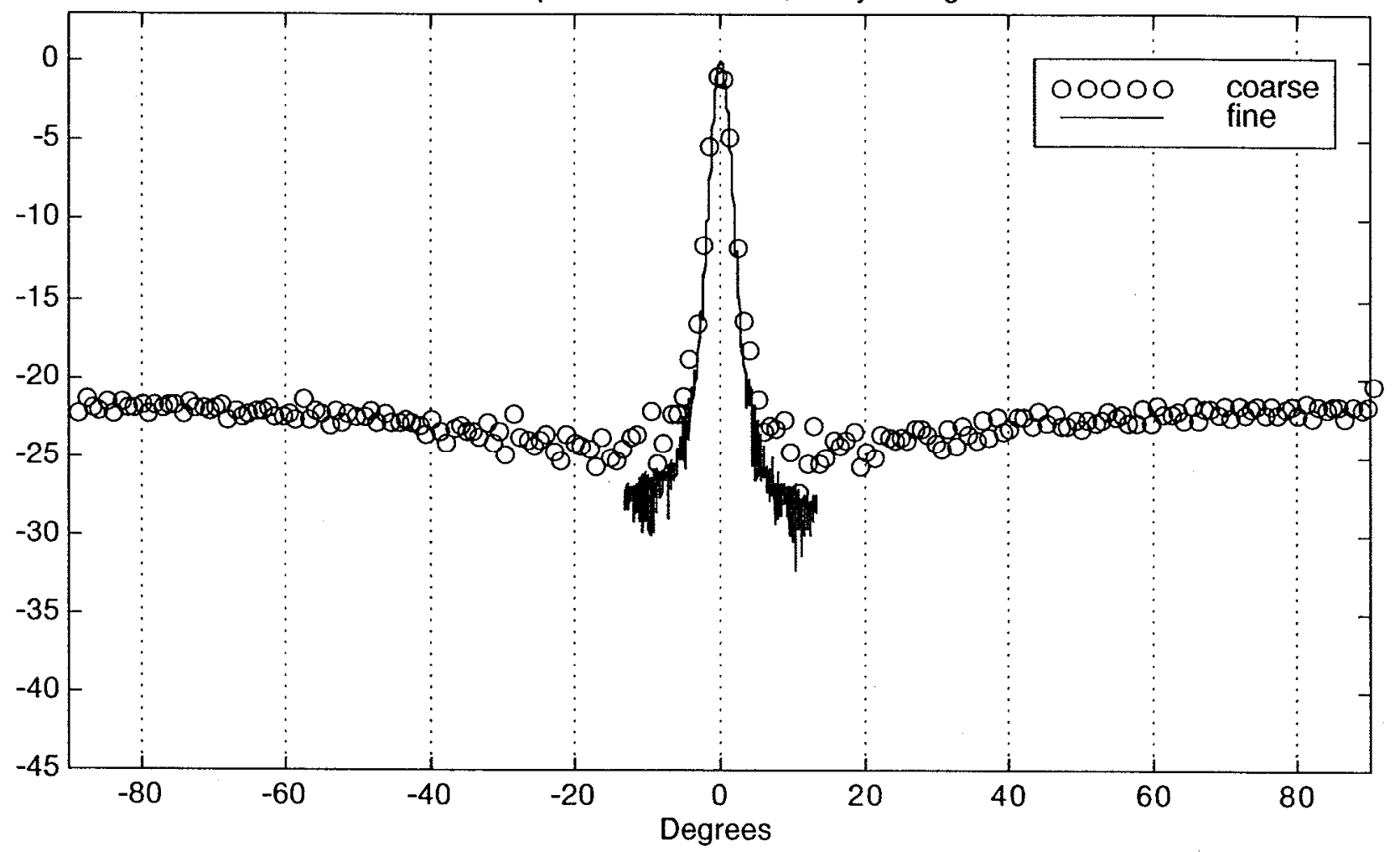

Figure E1-23. Beam pattern for SG1 with no compensation at a 600 foot range. Above is -90 to +90 degrees, below is -5 to +5 degrees. Amplitudes are in $\mathrm{dB}$.

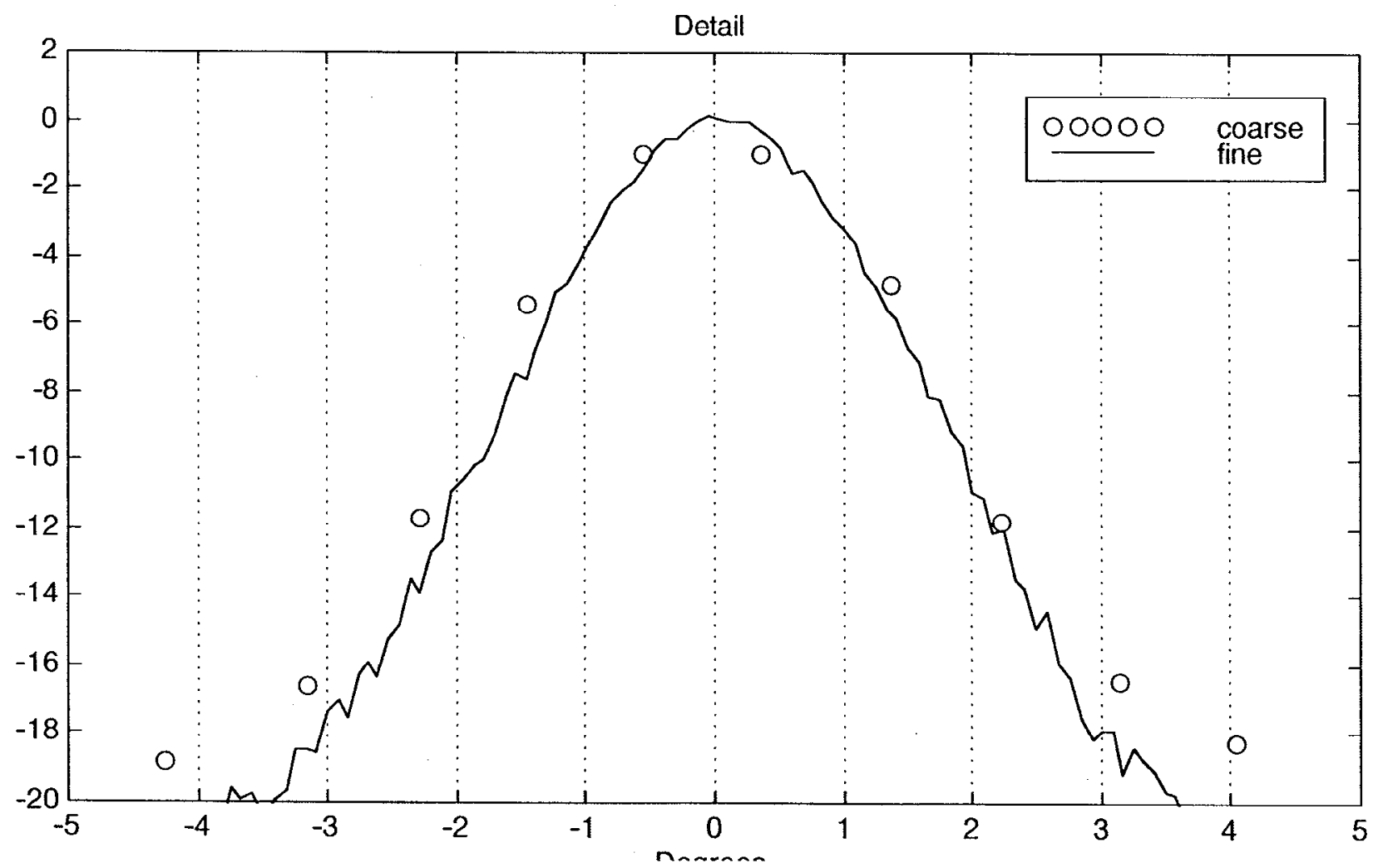


Beam pattern for SG1 NC piston, 200 yd. range

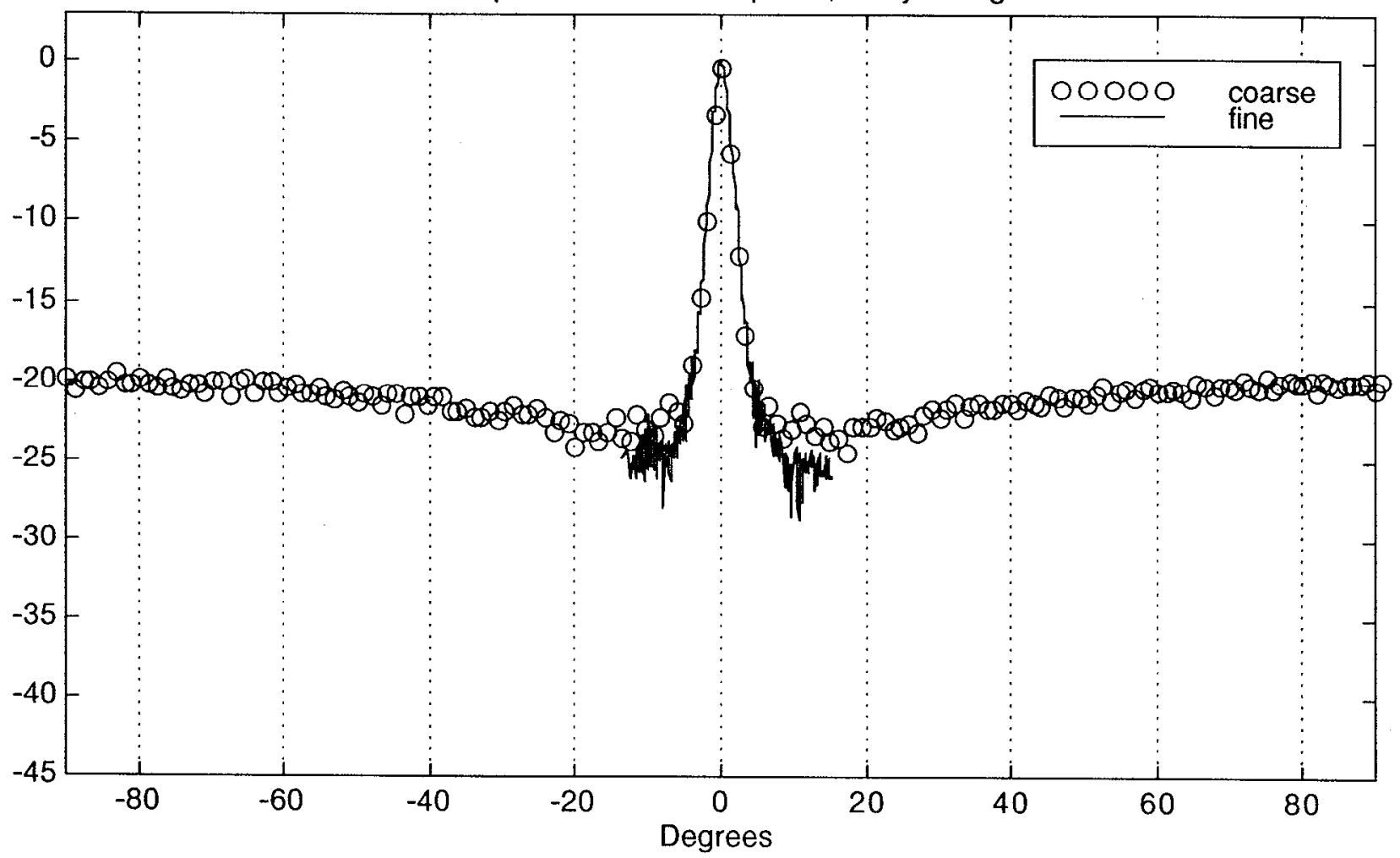

Figure E1-24. Beam pattern for SG1 piston drive with no compensation at a 600 foot range. Above is -90 to +90 degrees, below is -5 to +5 degrees. Amplitudes are in $\mathrm{dB}$.

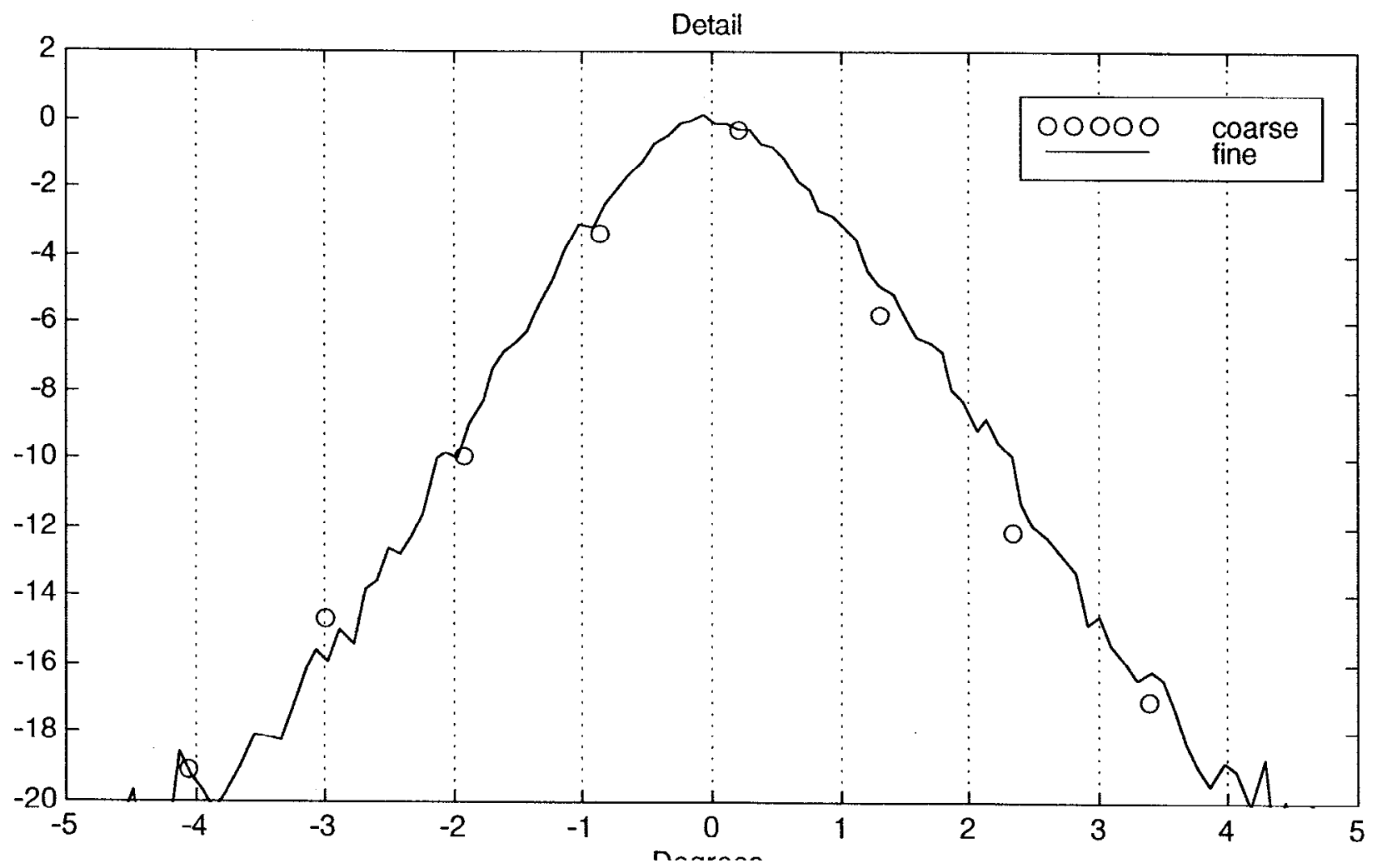

Appendix E1 
Beam pattern for $20 \mathrm{kHz}$ tone burst, $100 \mathrm{ft}$. range

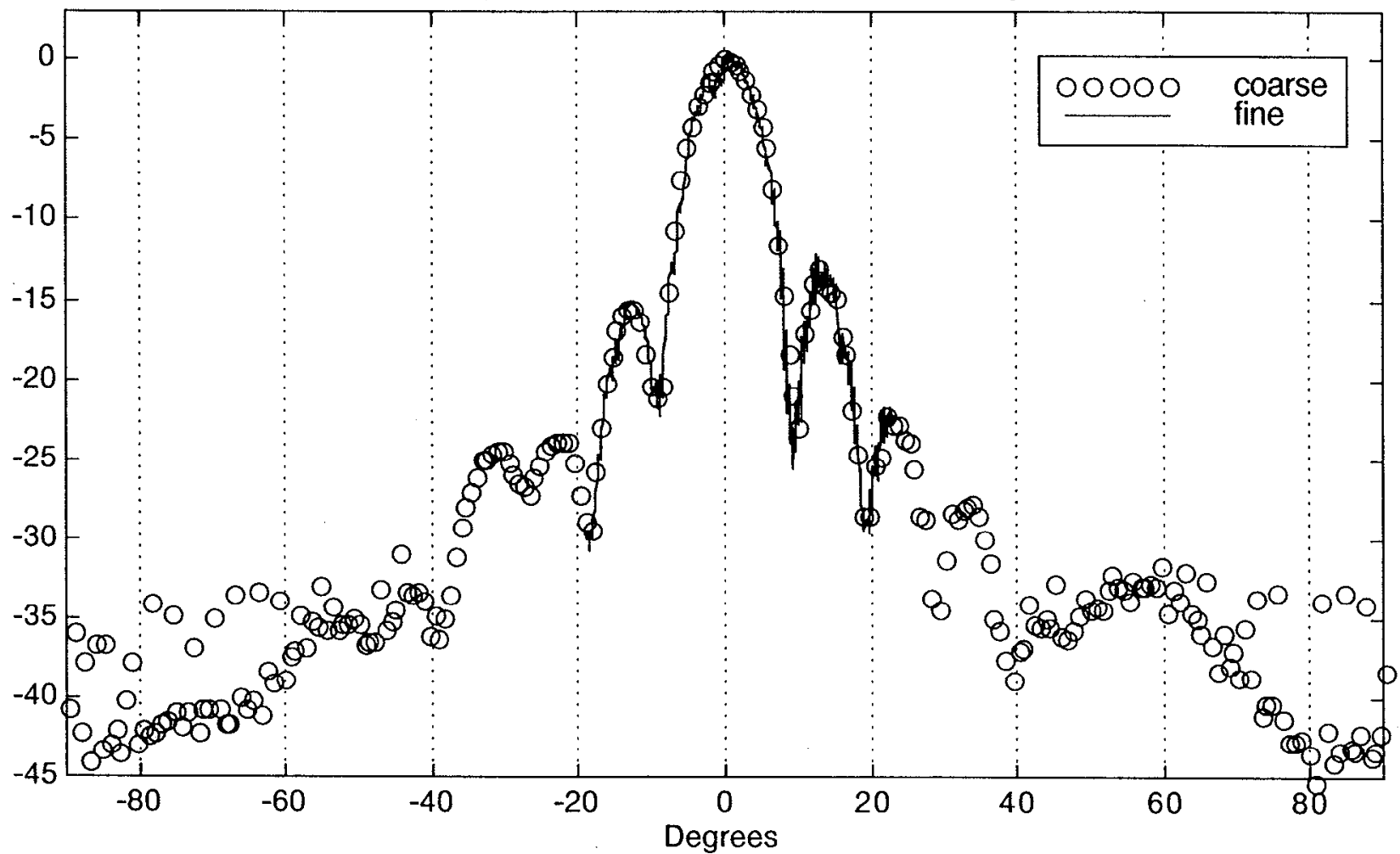

Figure E1-25. Beam pattern for $20 \mathrm{kHz}$ tone burst piston drive at a 100 foot range. Above is -90 to +90 degrees, below is -5 to +5 degrees. Amplitudes are in $\mathrm{dB}$.

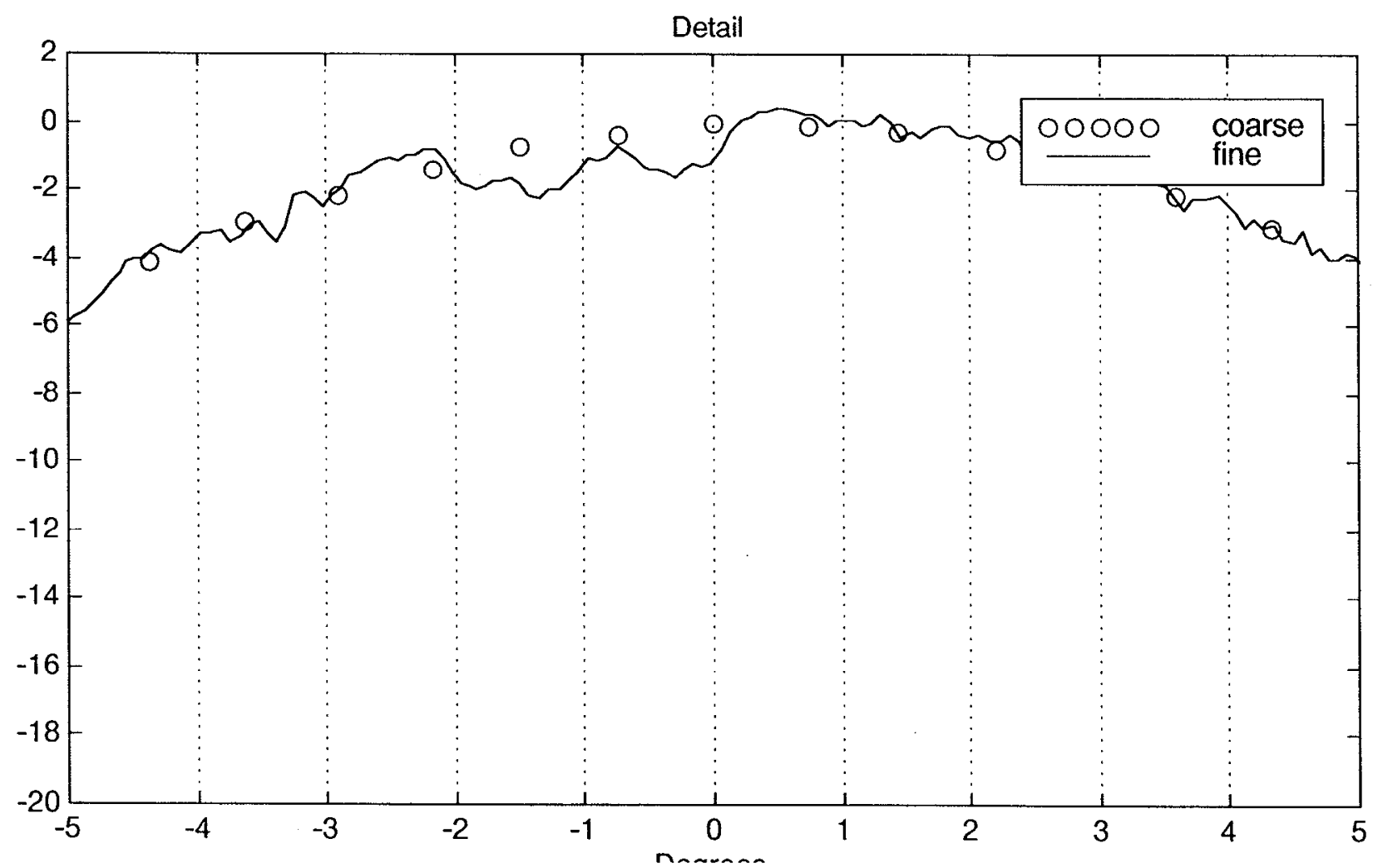


Beam pattern for $20 \mathrm{kHz}$ tone burst, $200 \mathrm{yd}$. range

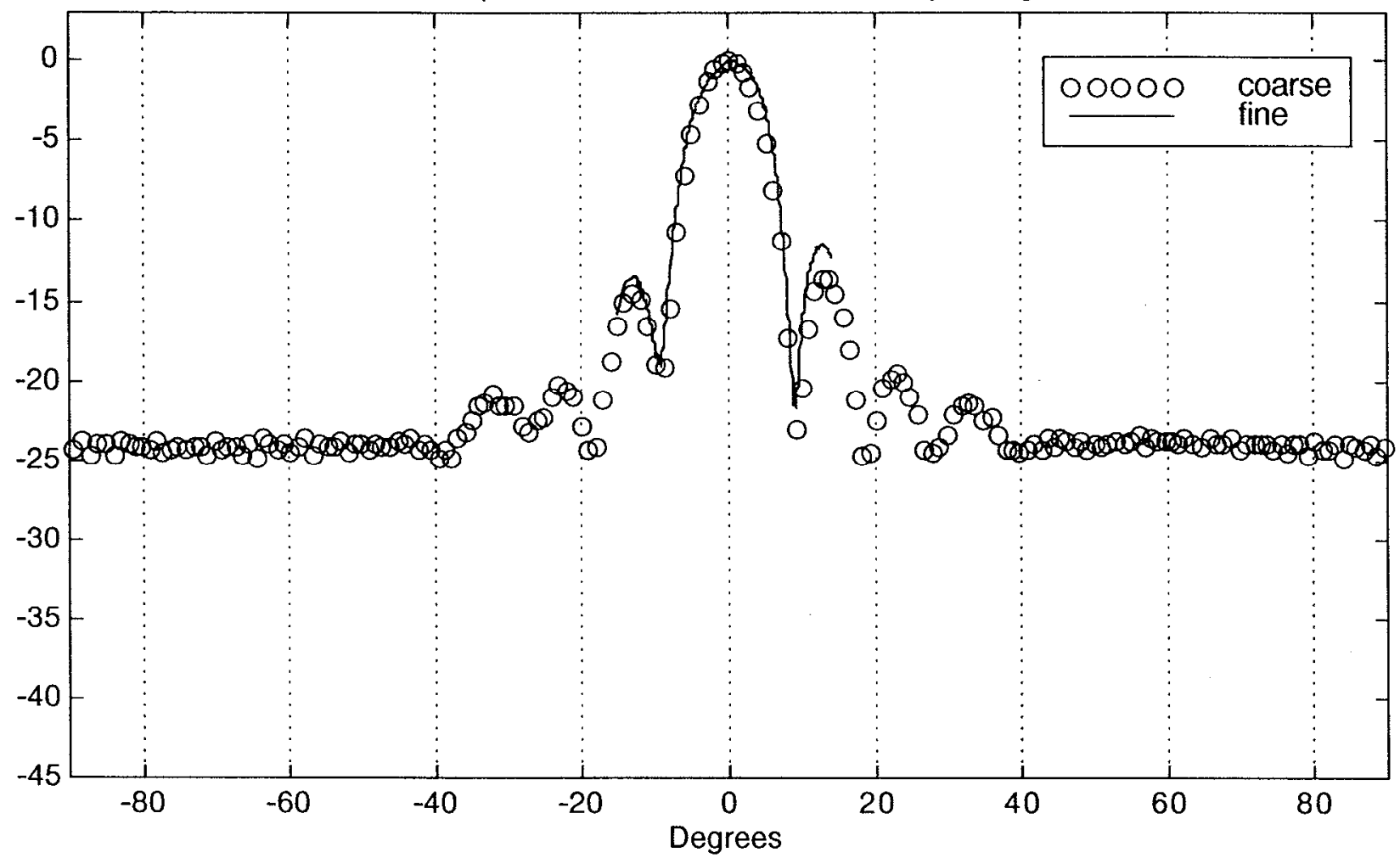

Figure E1-26. Beam pattern for $20 \mathrm{kHz}$ tone burst piston drive at a 600 foot range. Above is -90 to +90 degrees, below is -5 to +5 degrees. Amplitudes are in $\mathrm{dB}$.

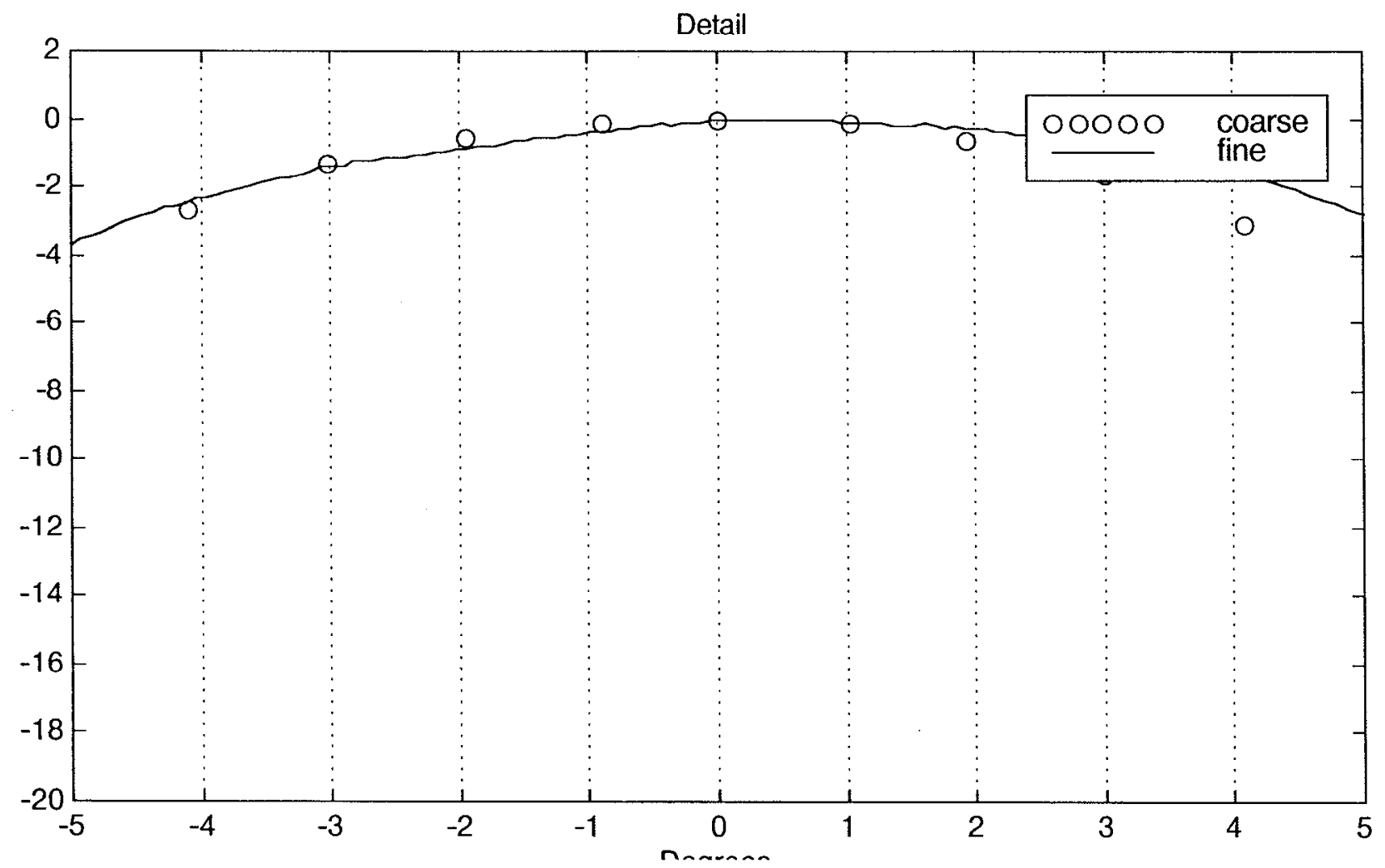


Beam pattern for $100 \mathrm{kHz}$ tone burst, $100 \mathrm{ft}$. range

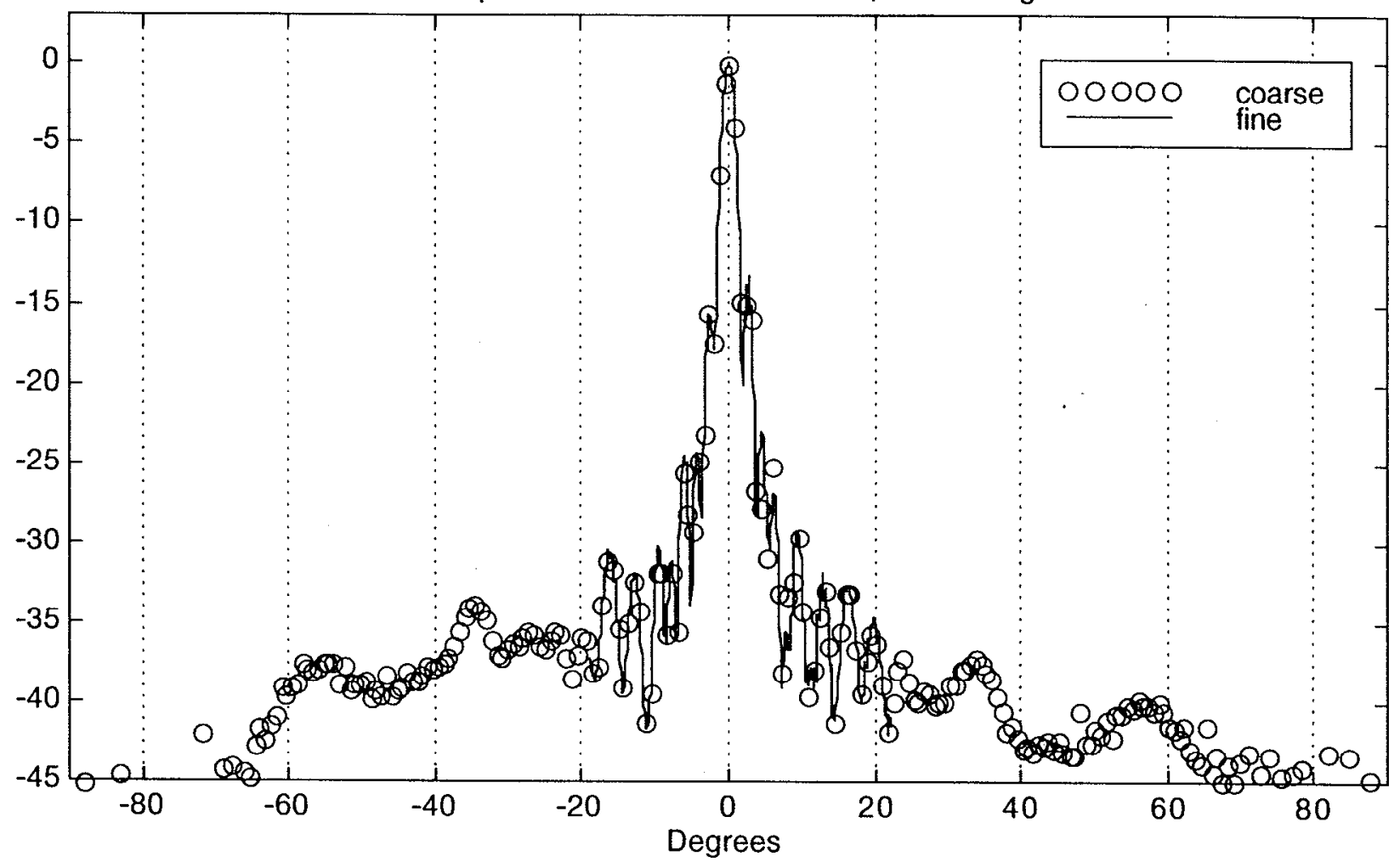

Figure $\mathrm{E} 1-27$. Beam pattern for $100 \mathrm{kHz}$ tone burst piston drive at a 100 foot range. Above is -90 to +90 degrees, below is -5 to +5 degrees. Amplitudes are in $\mathrm{dB}$.

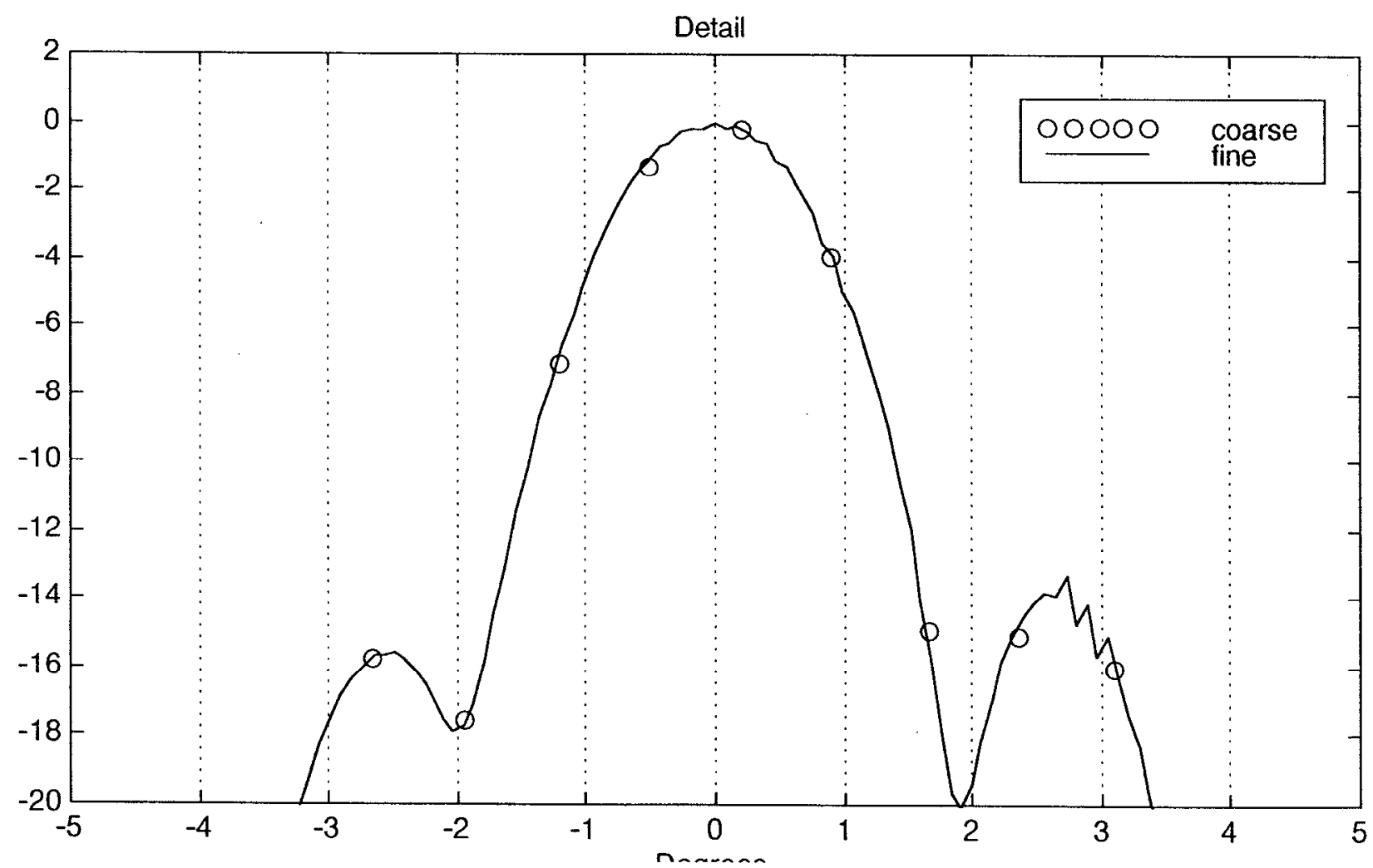


Beam pattern for $100 \mathrm{kHz}$ tone burst, $200 \mathrm{yd}$. range

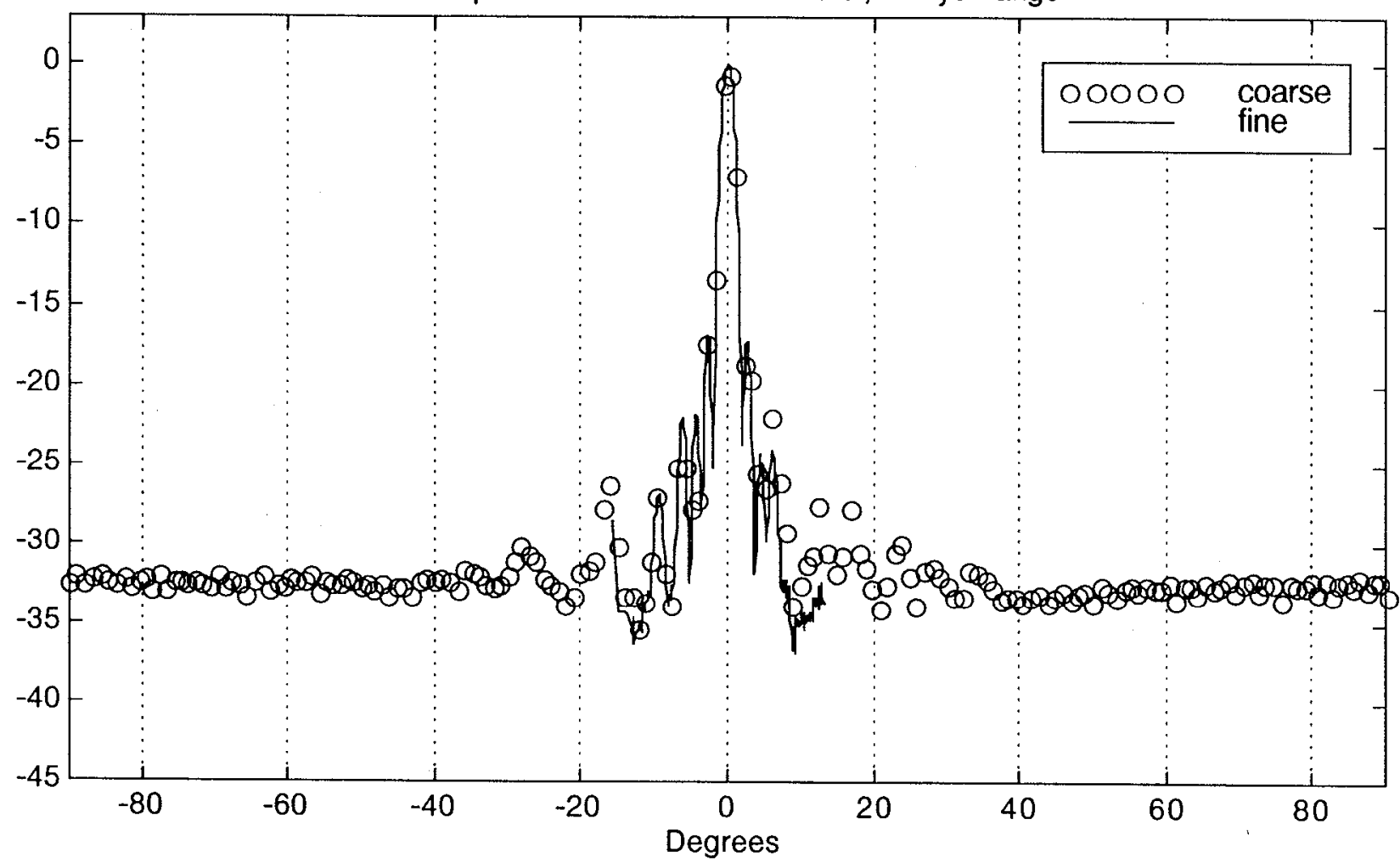

Figure $\mathrm{E} 1-28$. Beam pattern for $100 \mathrm{kHz}$ tone burst piston drive at a 600 foot range. Above is -90 to +90 degrees, below is -5 to +5 degrees. Amplitudes are in $\mathrm{dB}$.

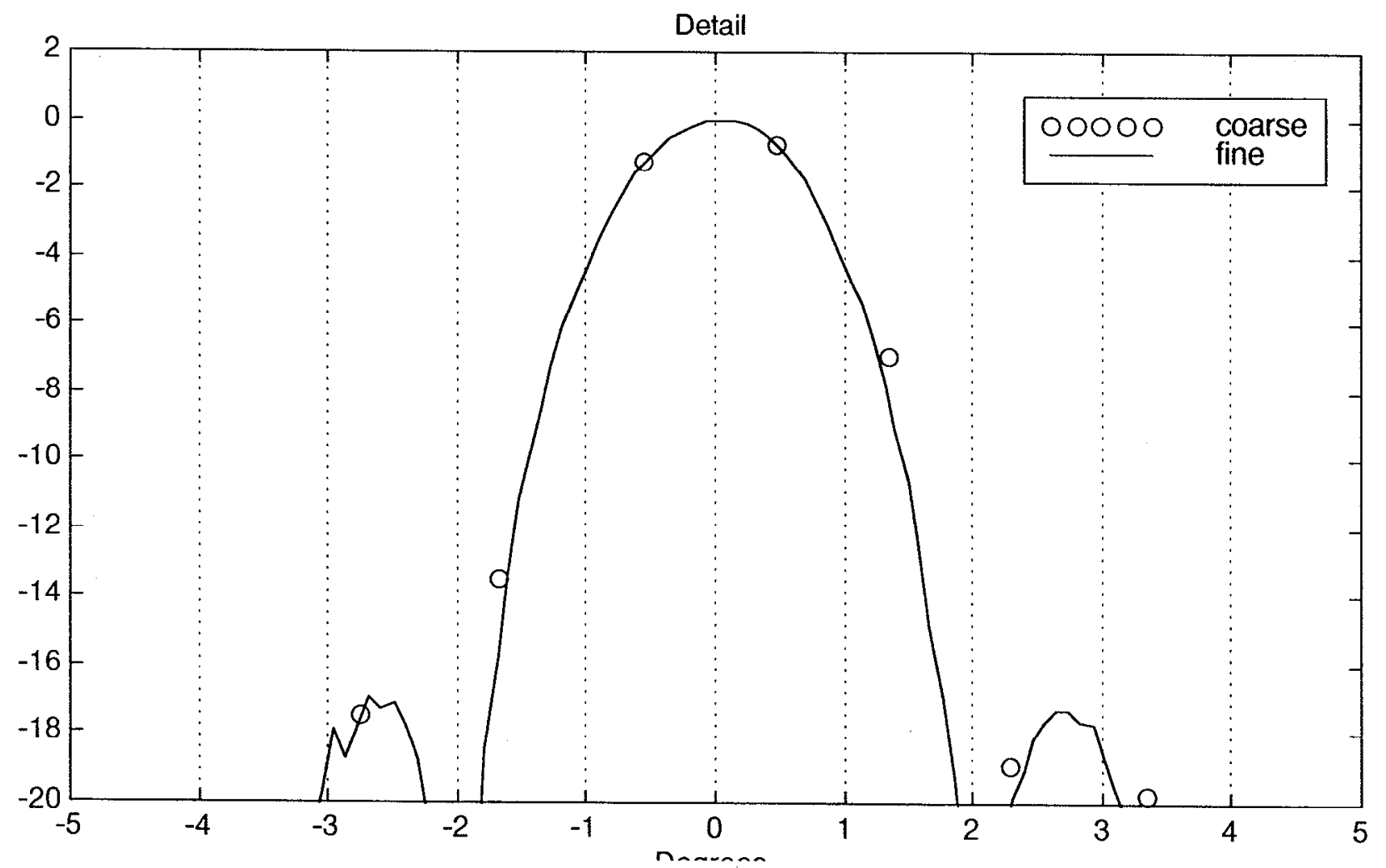


Beam pattern for $240 \mathrm{kHz}$ tone burst, $100 \mathrm{ft}$. range

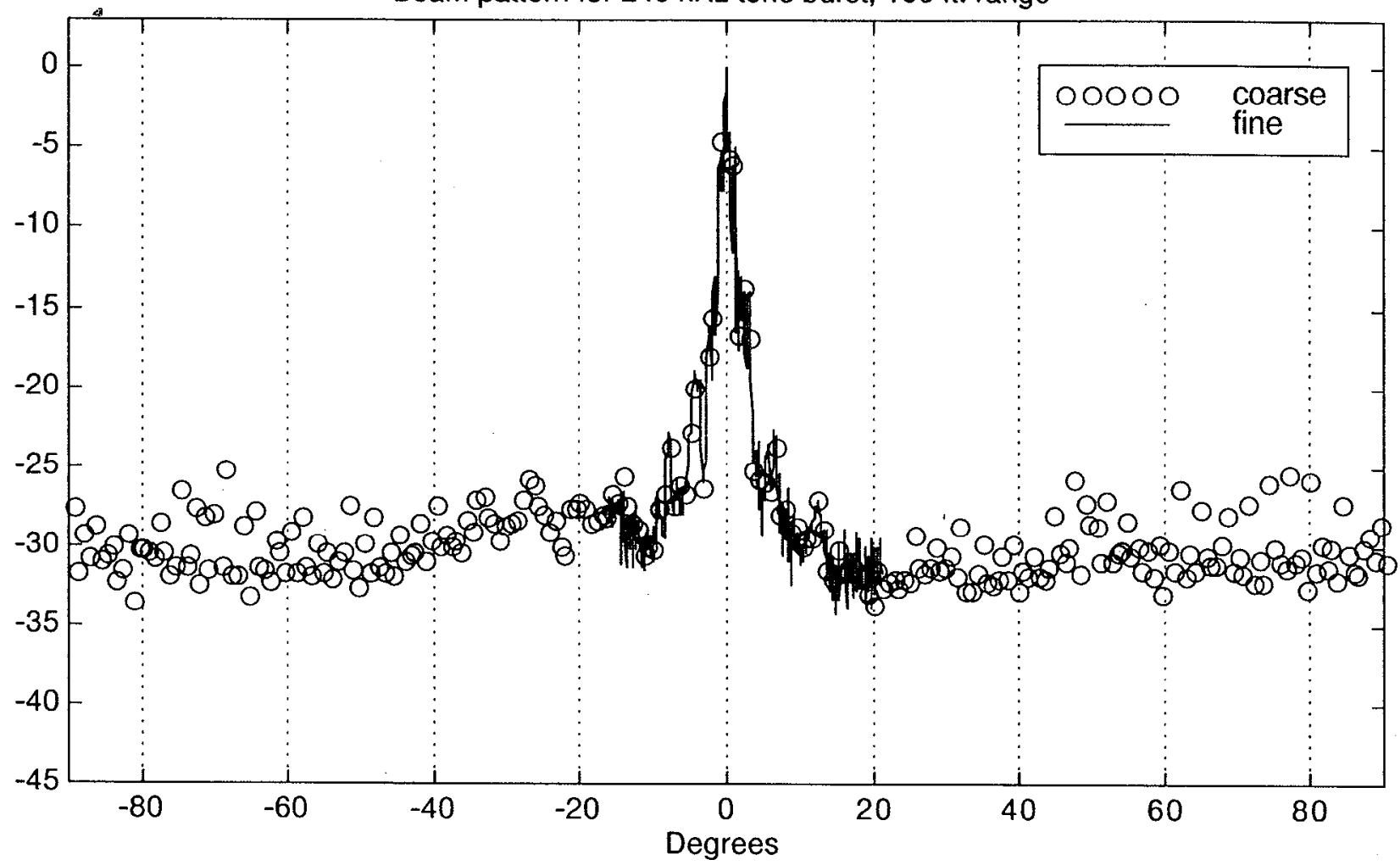

Figure E1-29. Beam pattern for $240 \mathrm{kHz}$ tone burst piston drive at a 100 foot range. Above is -90 to +90 degrees, below is -5 to +5 degrees. Amplitudes are in $\mathrm{dB}$.

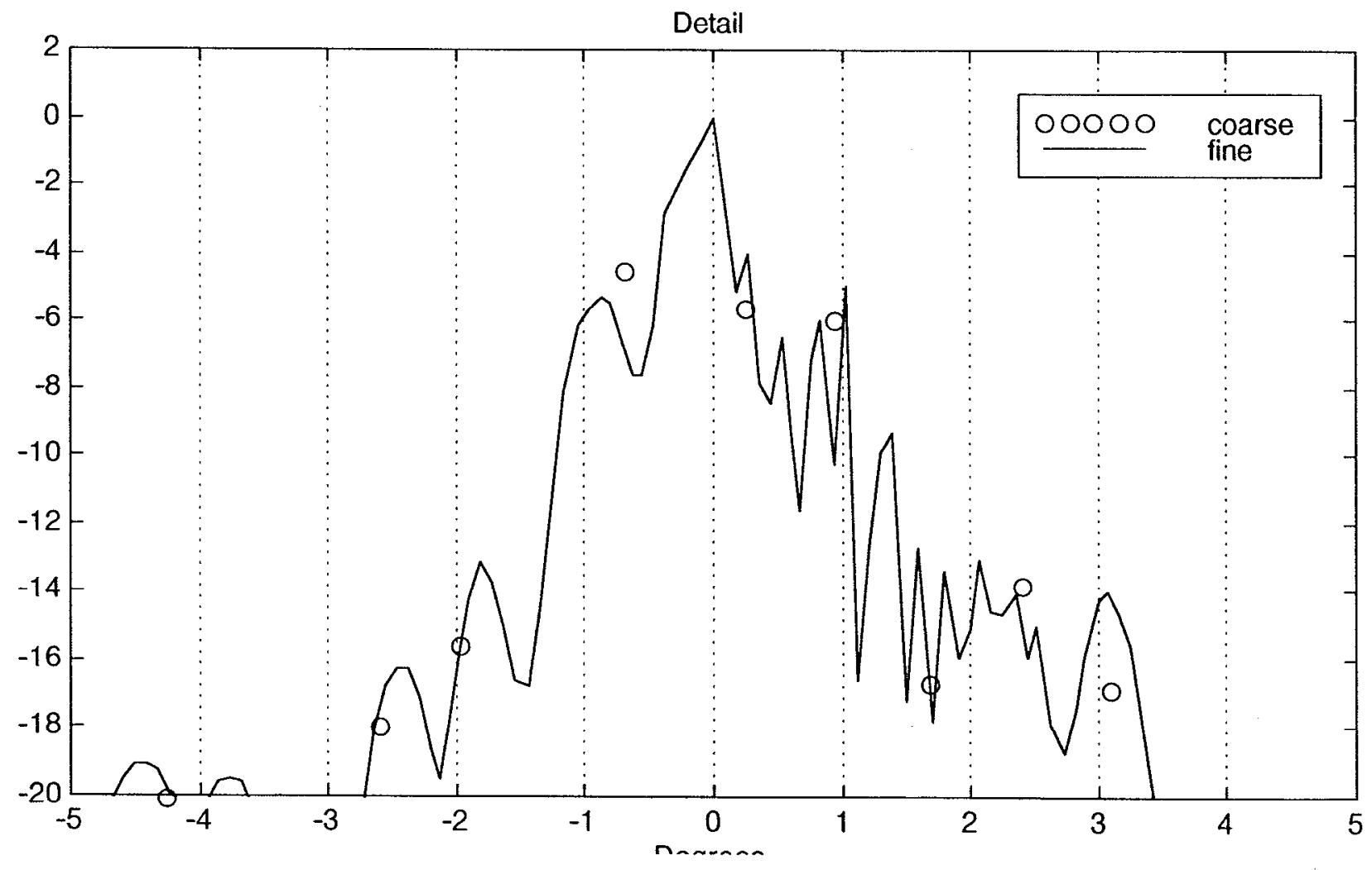


Beam pattern for $240 \mathrm{kHz}$ tone burst, $200 \mathrm{yd}$. range

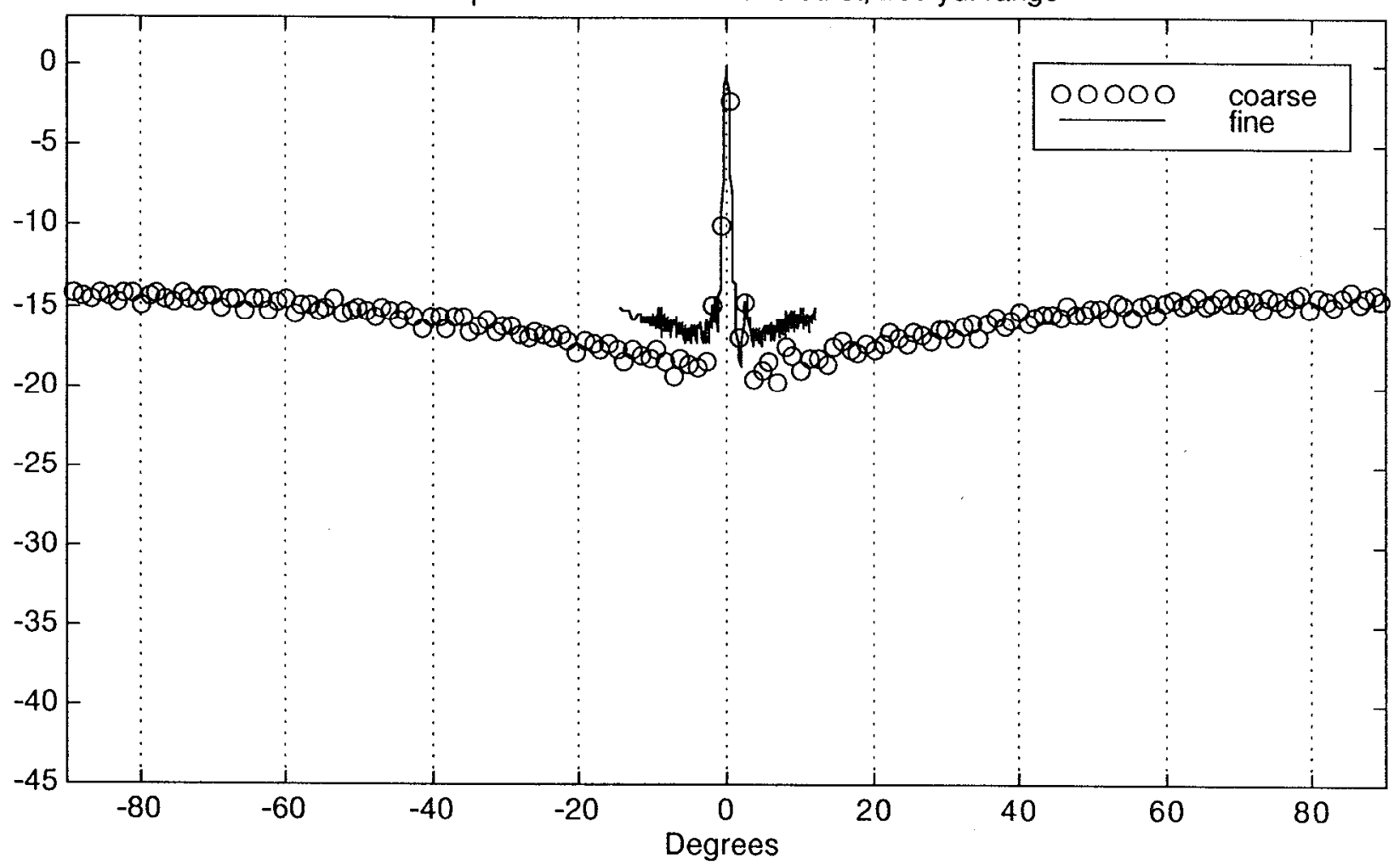

Figure $\mathrm{E} 1-30$. Beam pattern for $240 \mathrm{kHz}$ tone burst piston drive at a 600 foot range. Above is -90 to +90 degrees, below is -5 to +5 degrees. Amplitudes are in $\mathrm{dB}$.

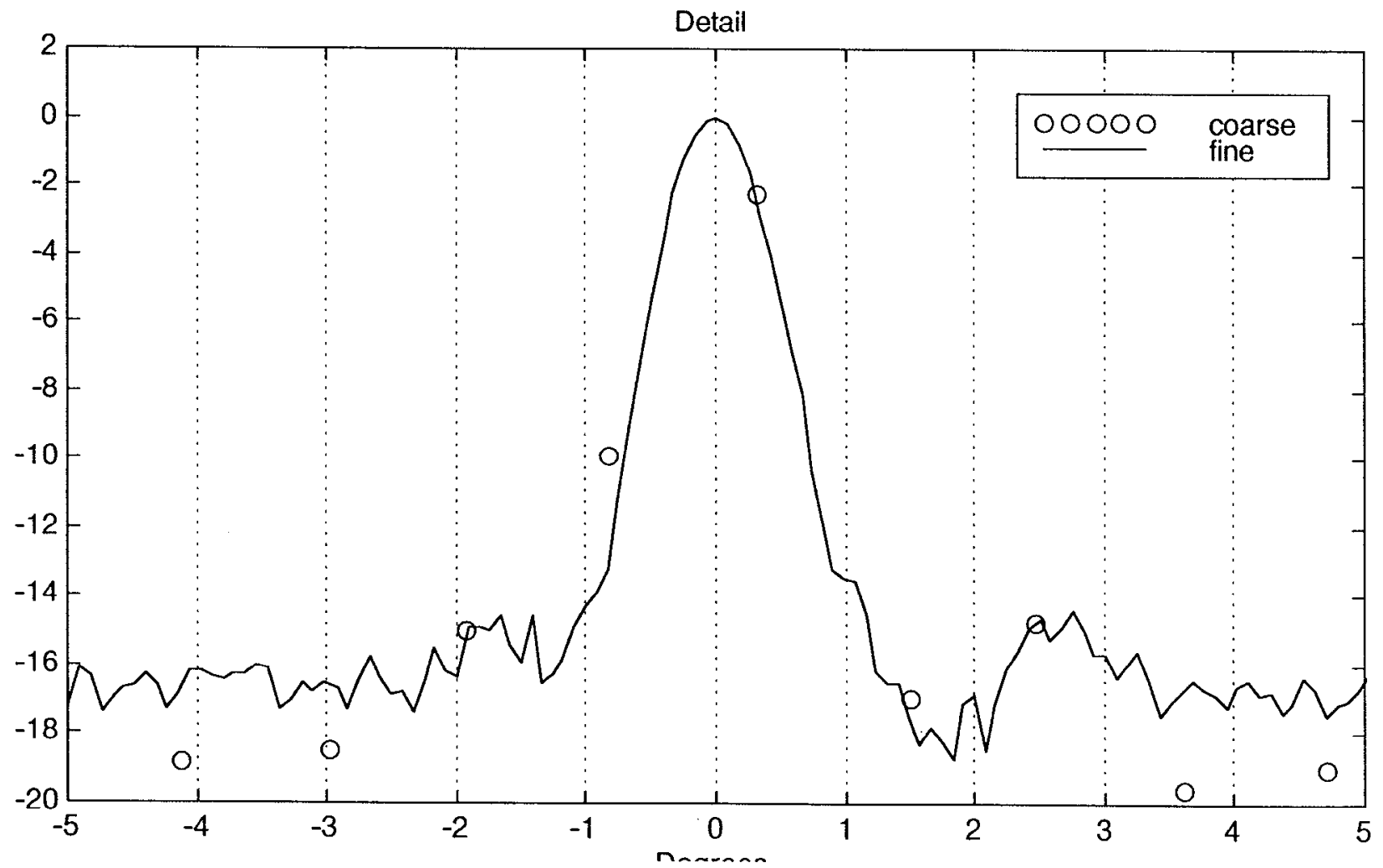




\section{IX.5 Appendix E: Beam Patterns}

The beam patterns of received data presented as polar plots, and linear angle plots. The scans represent data in two different measurements, a fine scale, small delta angle over a small range of angles, and a coarse scale, larger delta angle from -90 to +90 degrees. The magnitude of the plots is the energy in the beam at the given angle in $\mathrm{dB}$.

\section{Appendix E.1 Polar energy plots, 100 foot range data}

The beam shape is presented for two ranges, measurements at 100 feet with the array and receiver mounted on the barge, followed by the beam shape at 600 feet, with the array mounted on the barge and the receiver mounted on a tower anchored to the lake bottom. The beams were also launched as stretched pulse beams, and the results are plotted following each range. The plots are self explainatory.

\section{Appendix E.1.a 100 foot data, tone bursts.}

\section{Beam pattern for $20 \mathrm{kHz}$ tone burst, $100 \mathrm{ft}$. range}

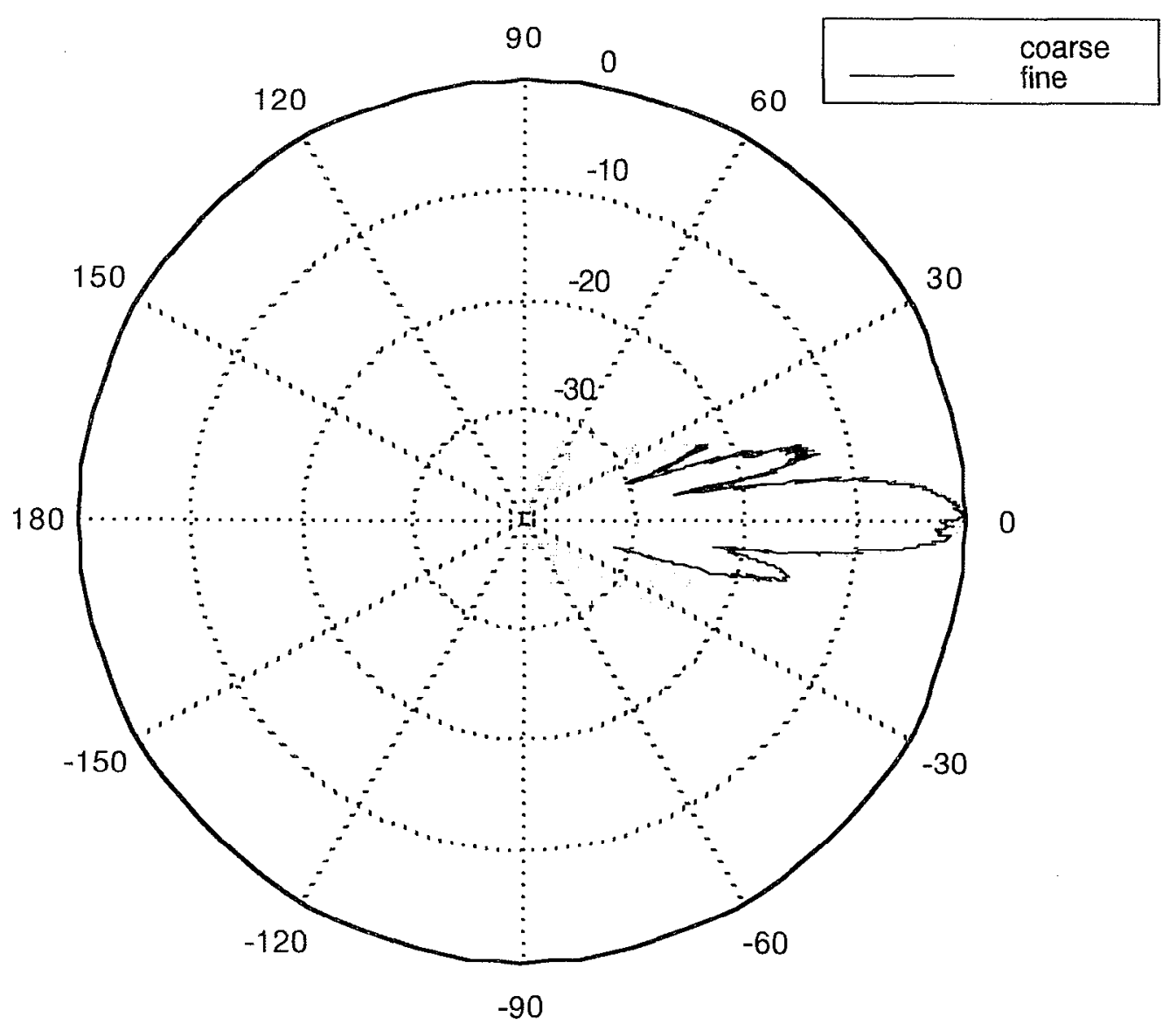


Beam pattern for $100 \mathrm{kHz}$ tone burst, $100 \mathrm{ft}$. range

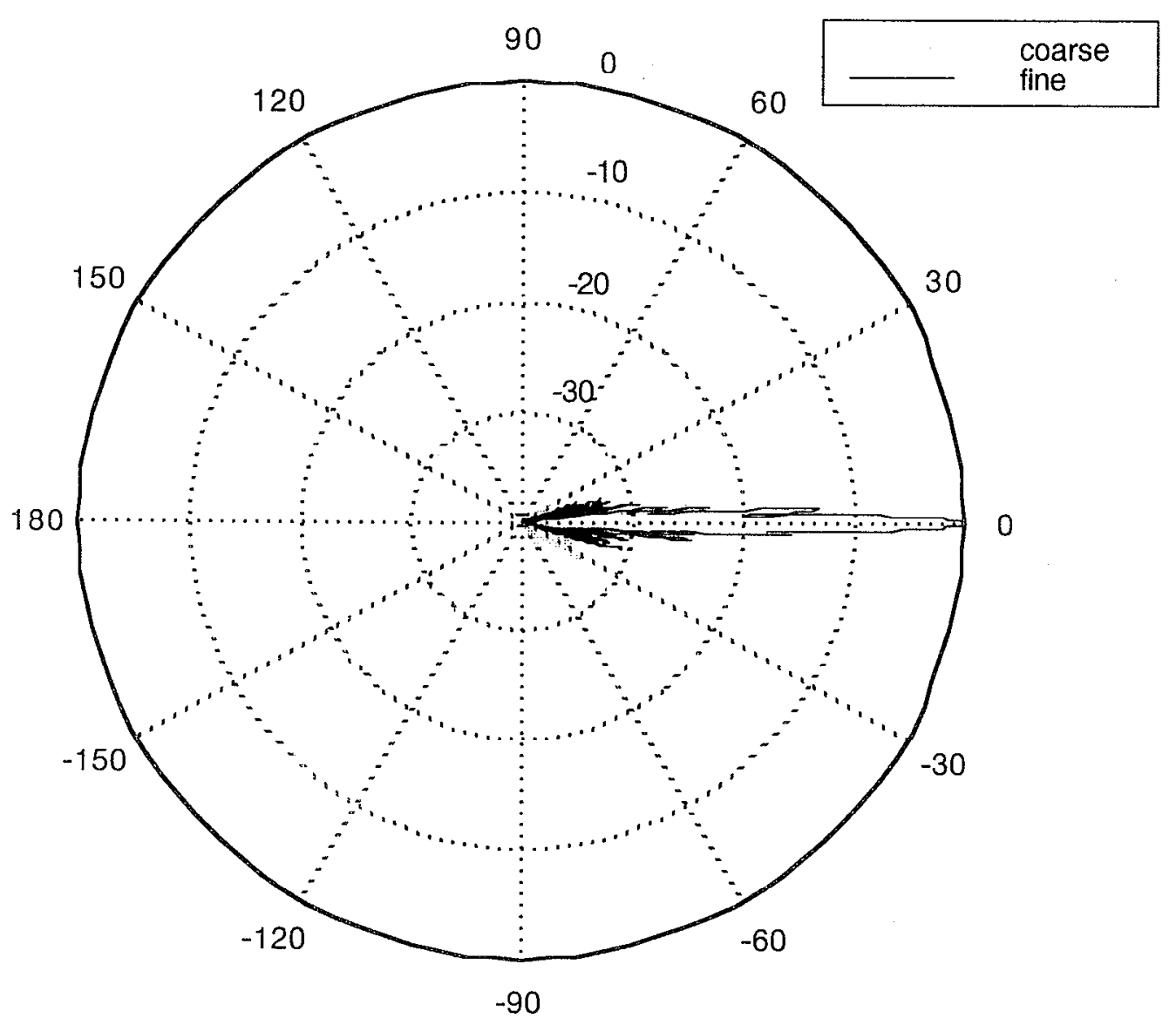


Beam pattern for $240 \mathrm{kHz}$ tone burst, $100 \mathrm{ft}$. range

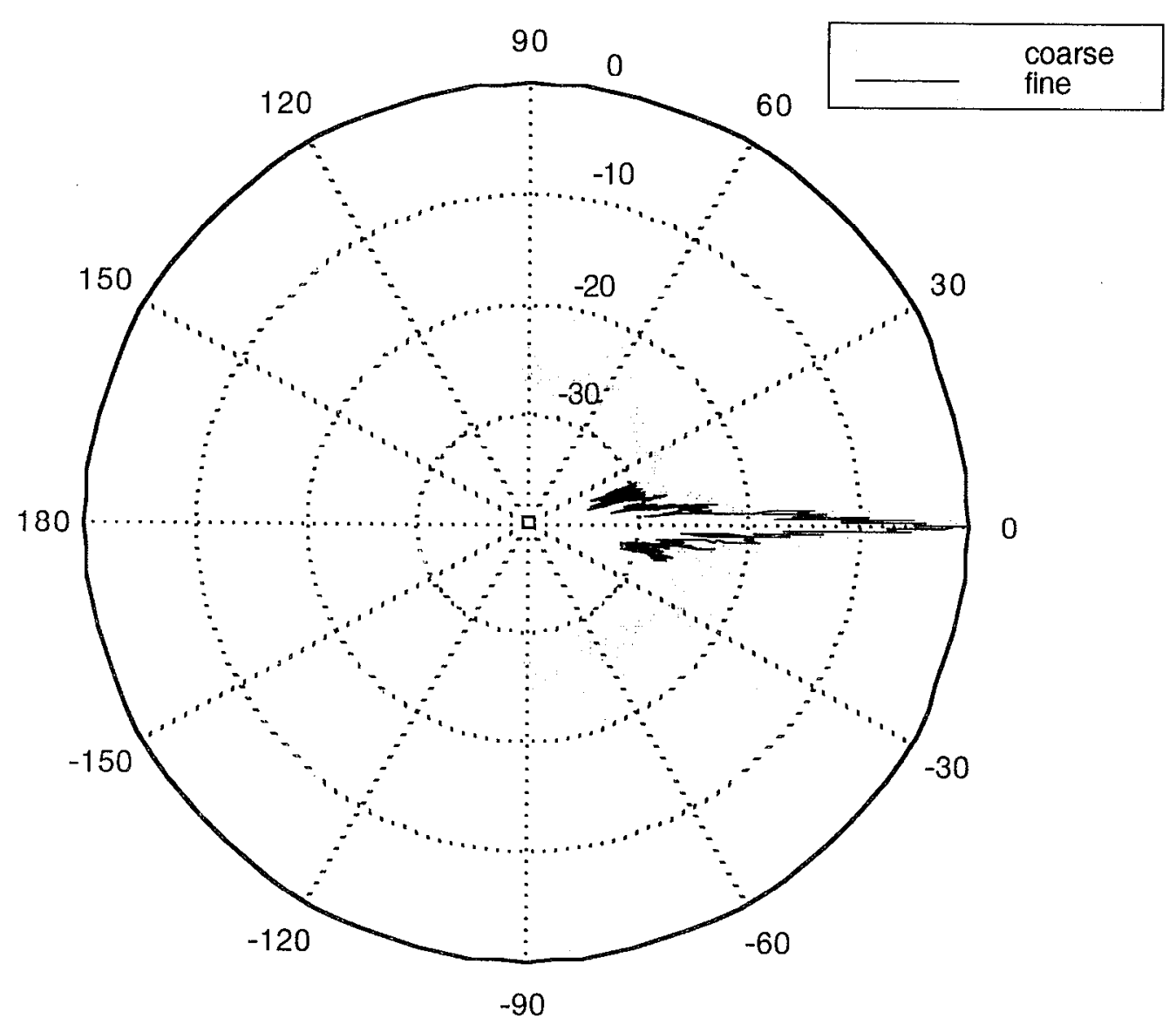




\section{Appendix E.1.b Pulse Data, 100 feet}

Beam pattern for MPS $1 \mathrm{NC}, 100 \mathrm{ft}$. range

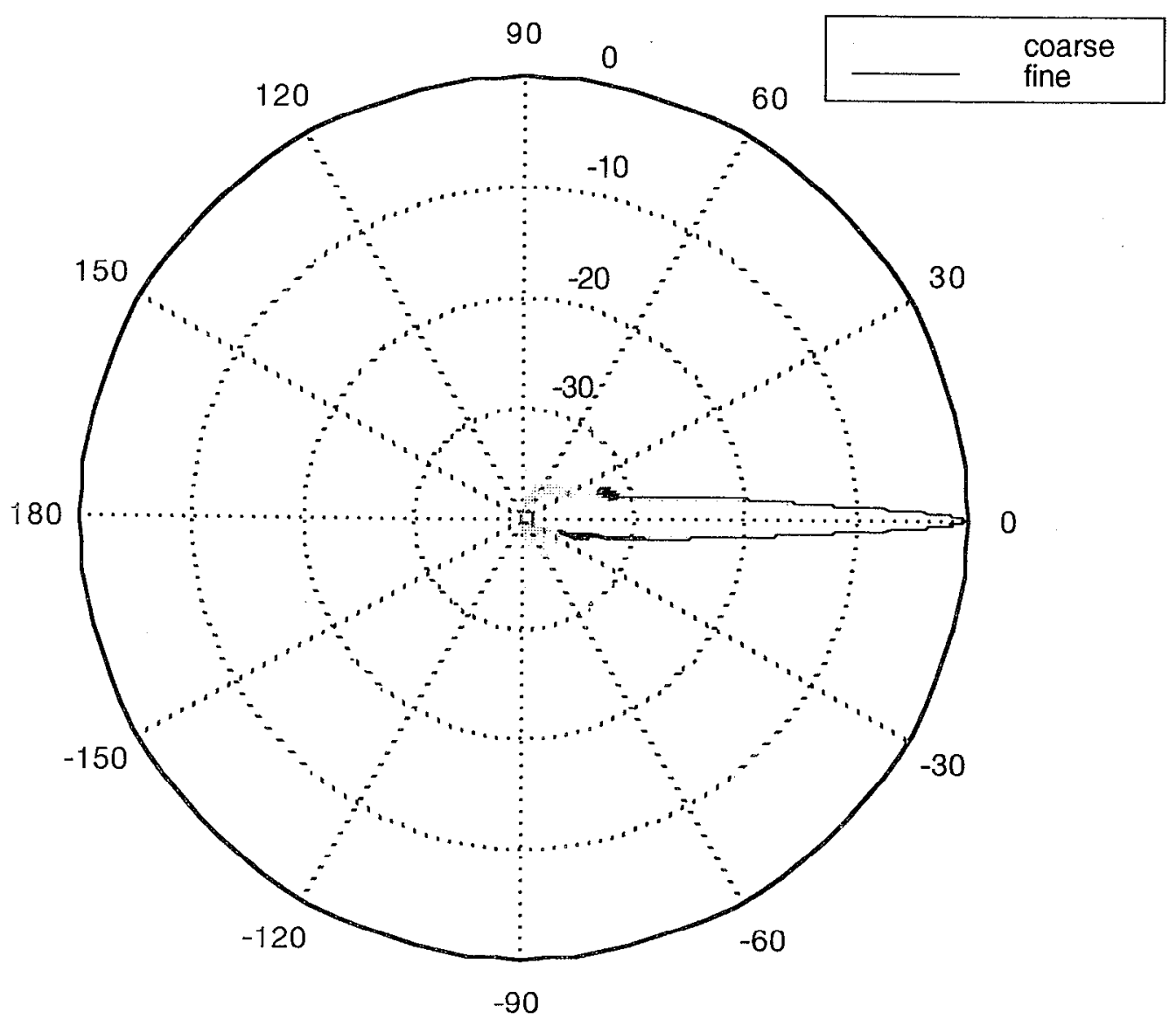


Beam pattern for MPS1 2D, $100 \mathrm{ft}$. range

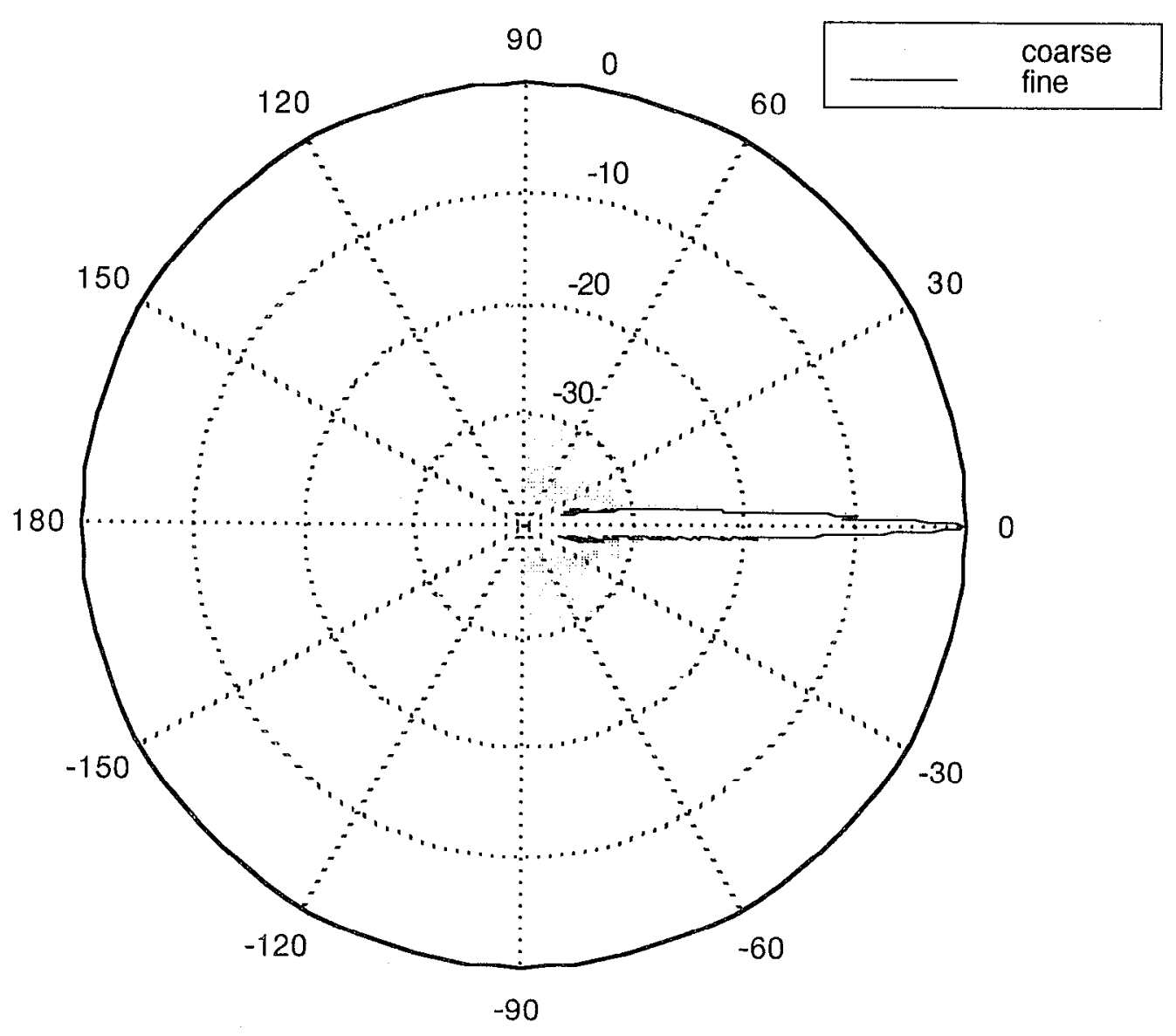




\section{Beam pattern for MPS1 3D, $100 \mathrm{ft}$. range}

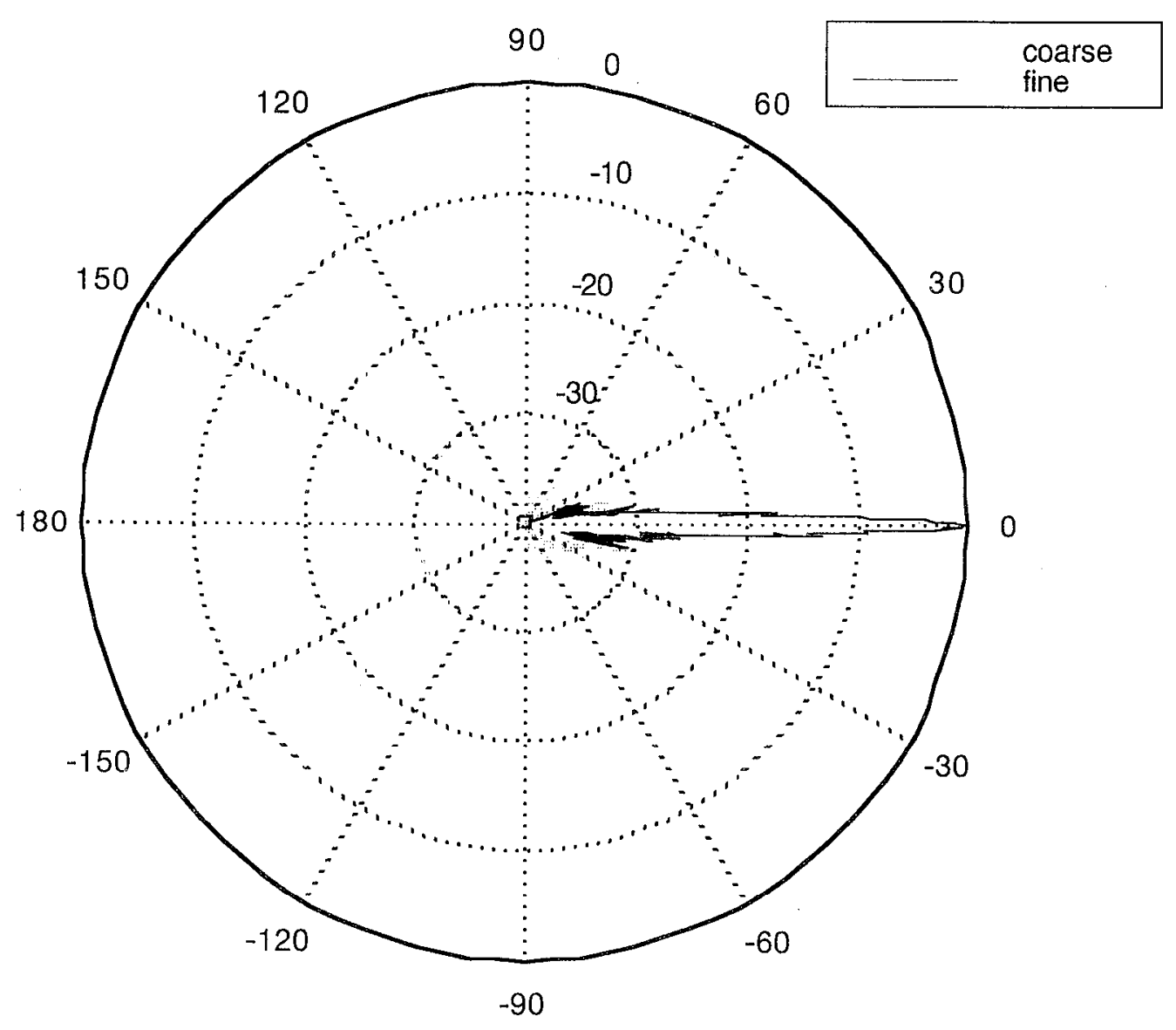




\section{Beam pattern for SG1 NC, $100 \mathrm{ft}$. range}

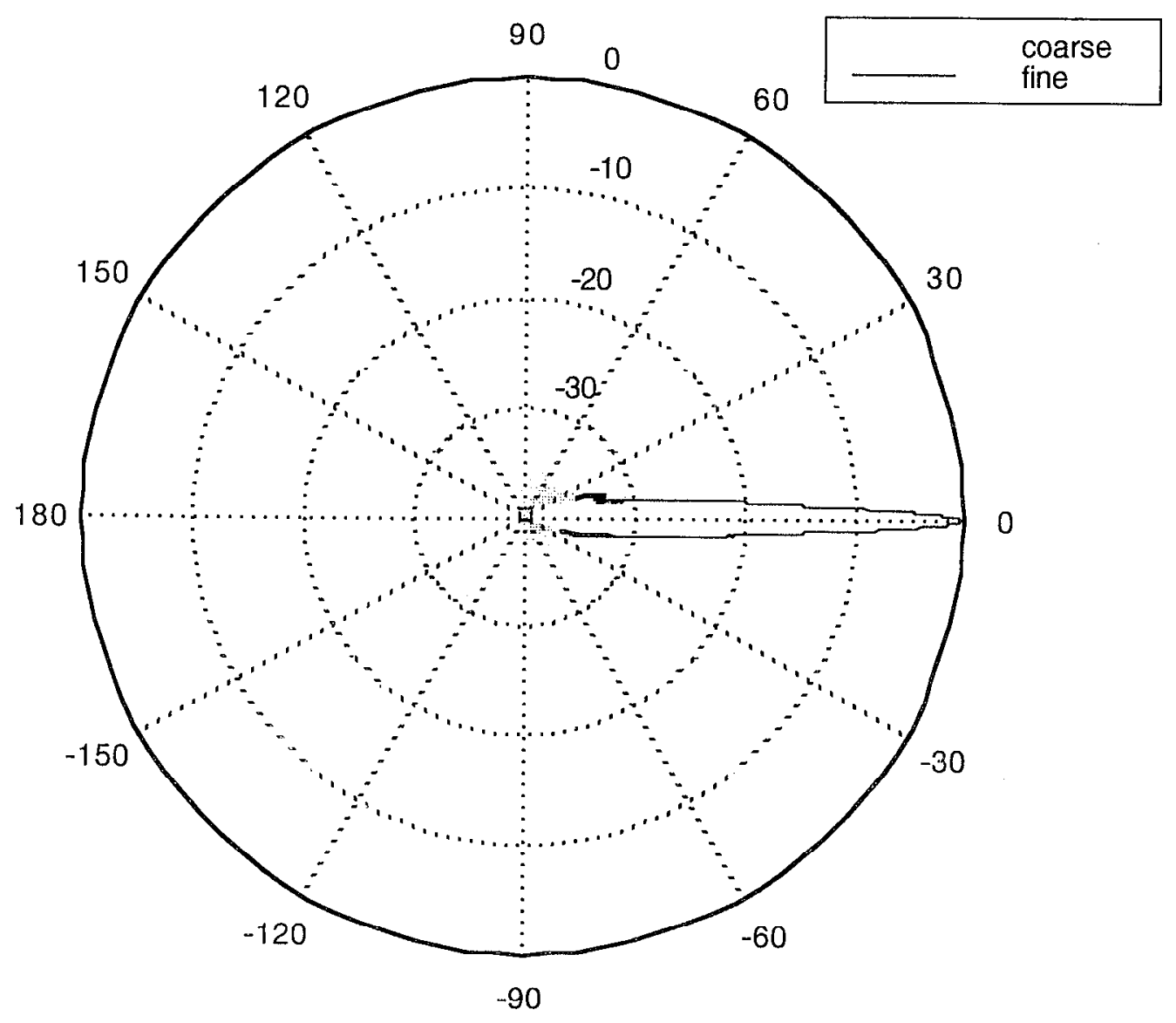




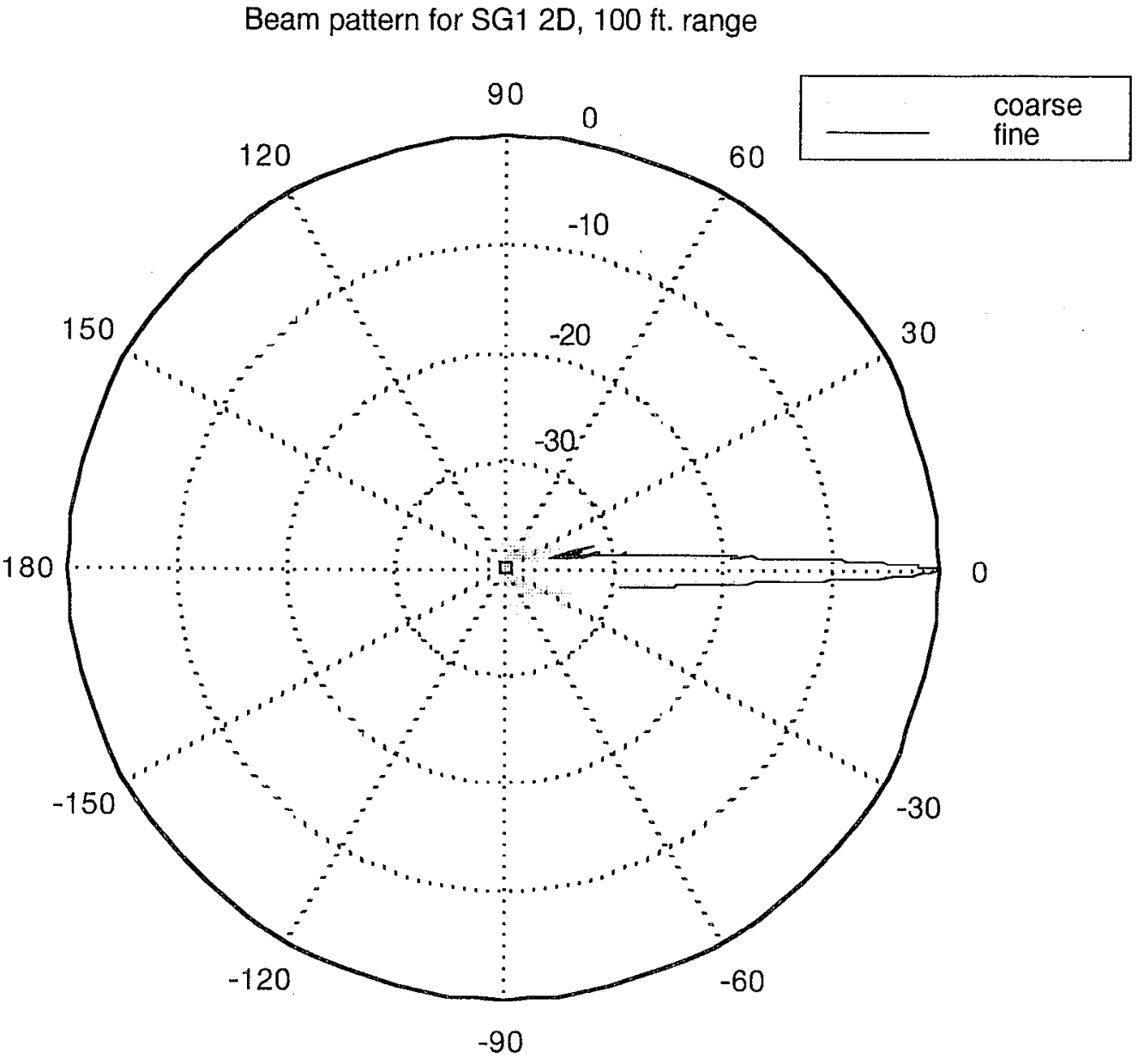




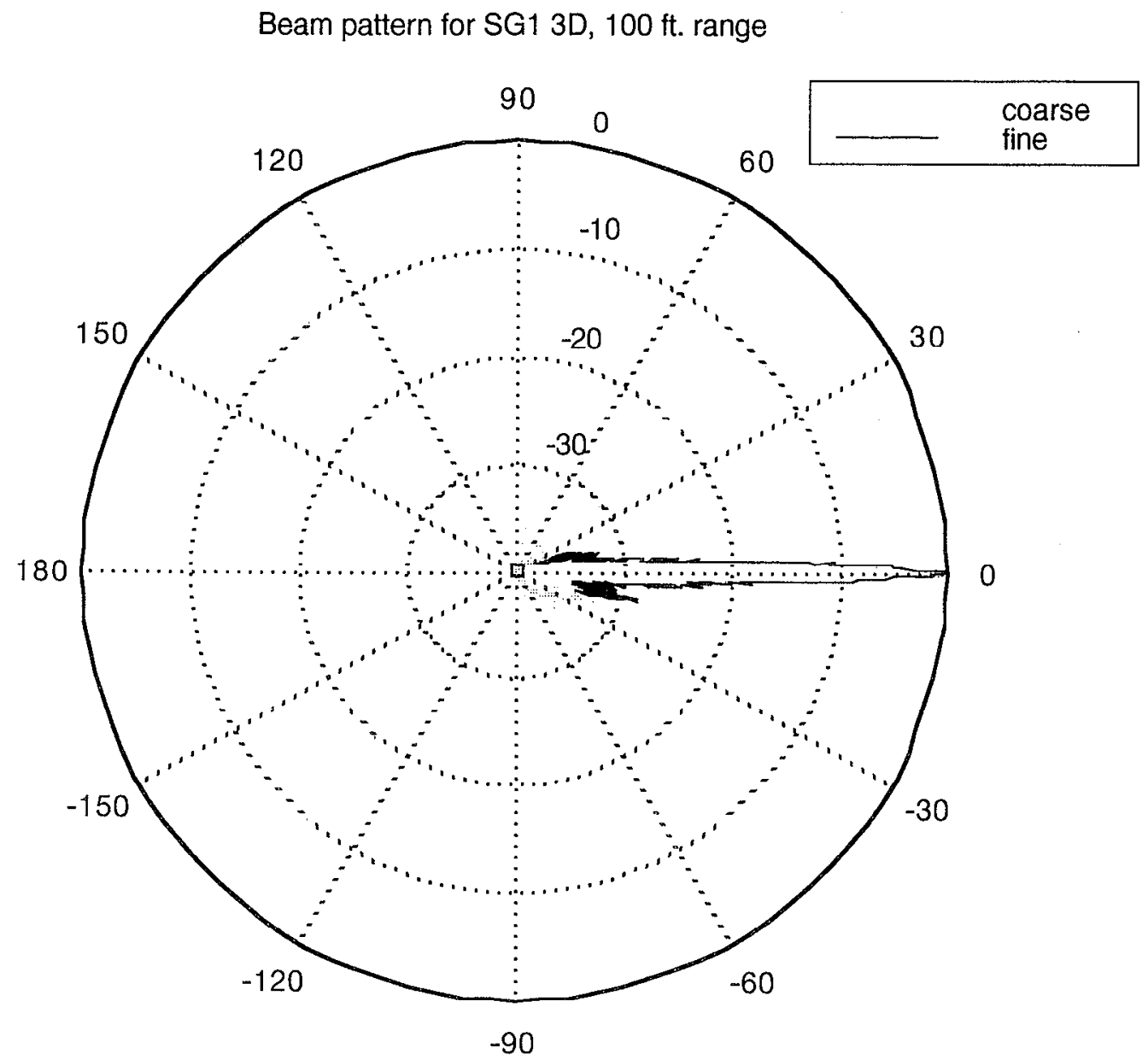




\section{Appendix E.1.c Piston pulse Beam Patterns}

Following are the beam patterns of the array driven as a piston, using the center signal from a Localized Wave pulse in all cases.

Beam pattern for MPS1 2D piston, $100 \mathrm{ft}$. range

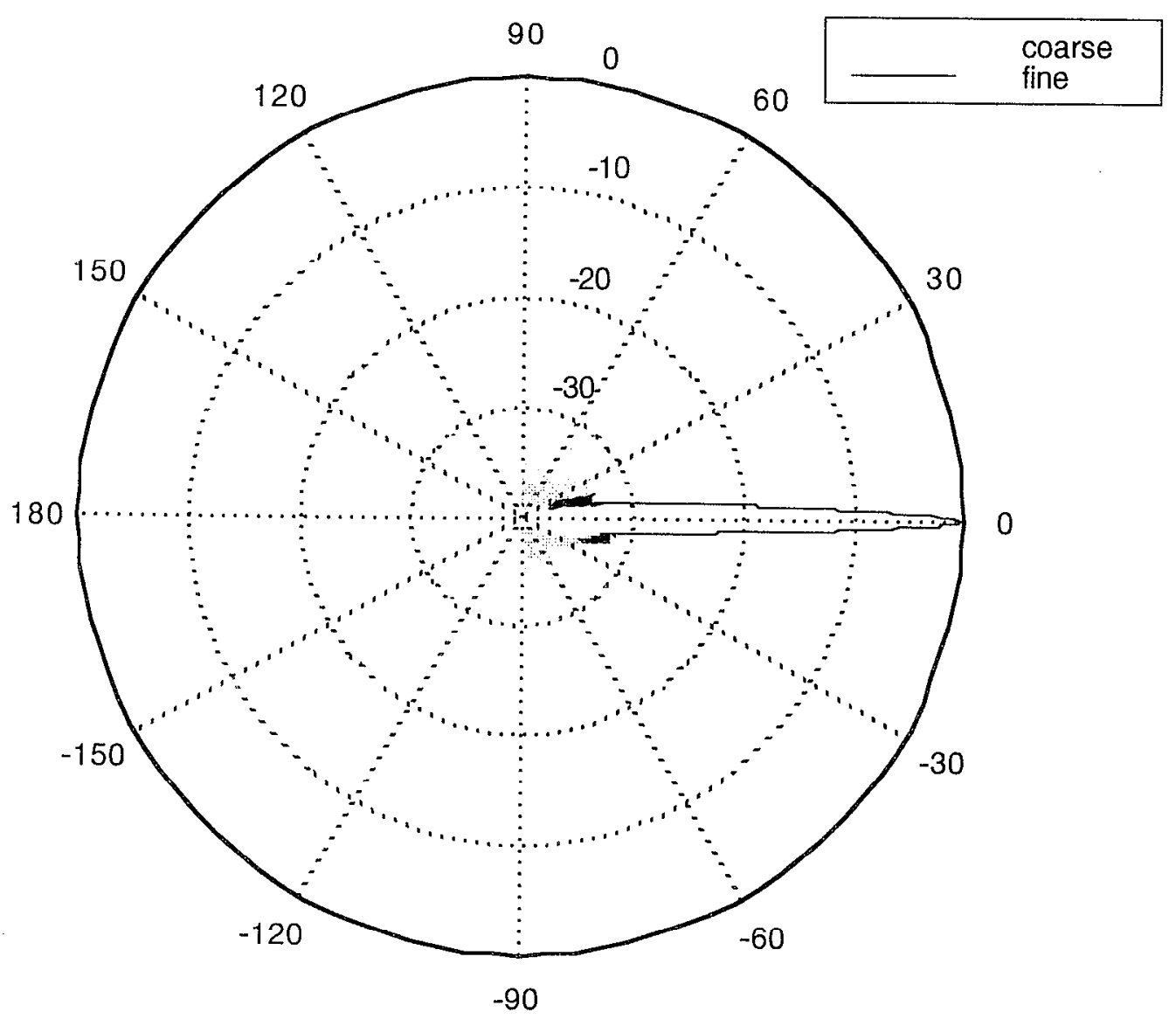


Beam pattern for MPS1 3D piston, $100 \mathrm{ft}$. range

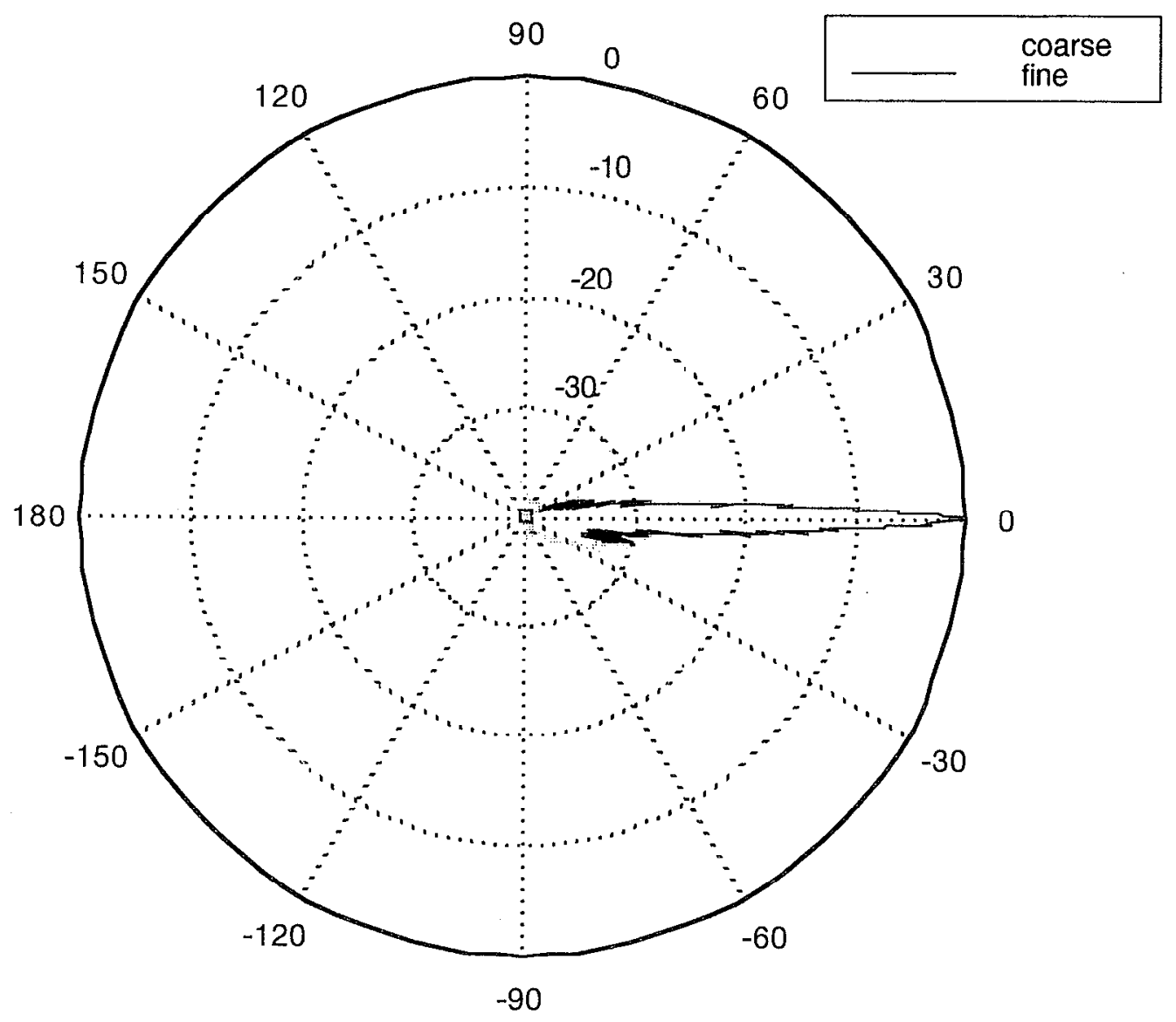


Beam pattern for SG1 2D piston, $100 \mathrm{ft}$. range

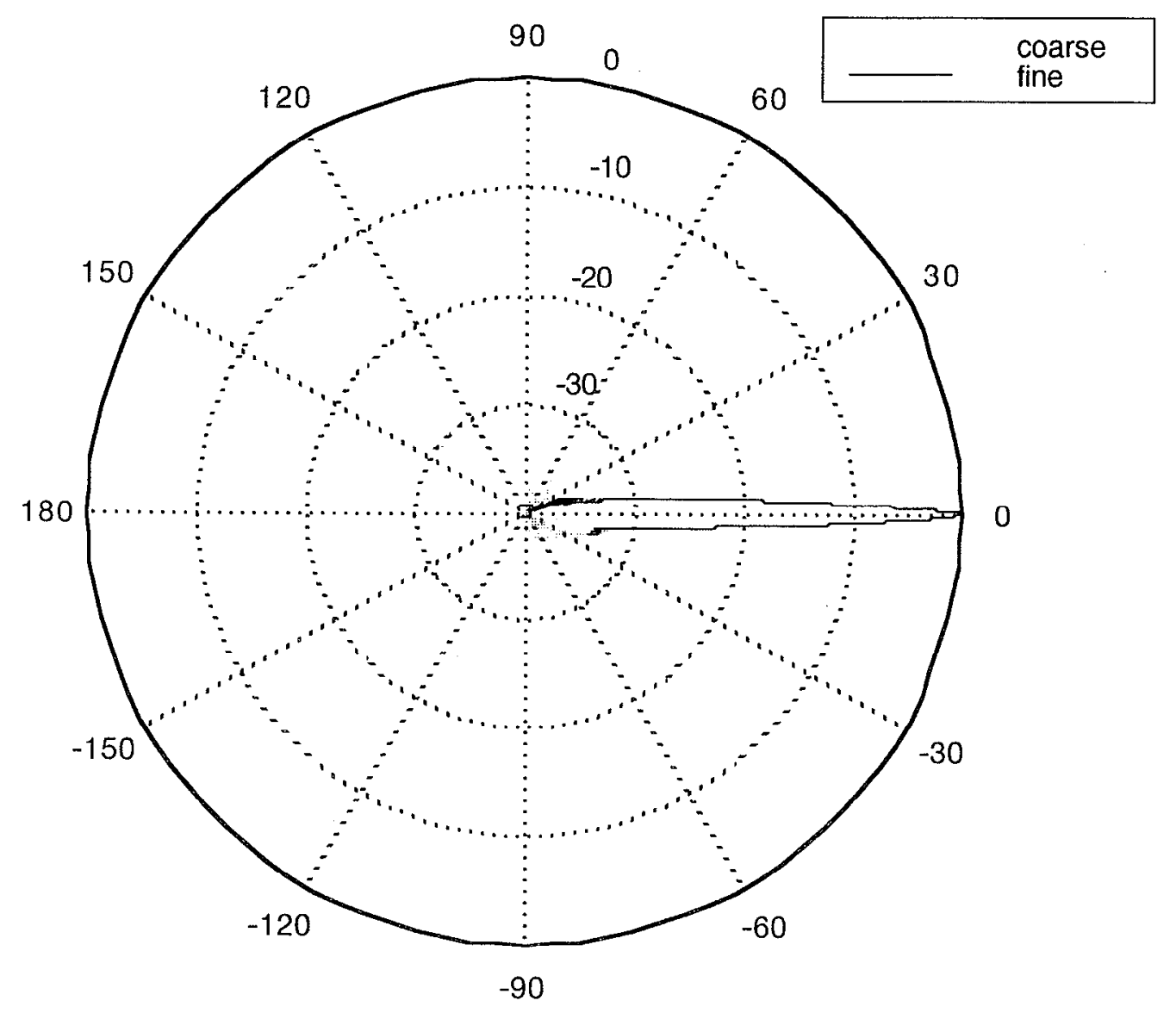


Beam pattern for SG1 3D piston, $100 \mathrm{ft}$. range

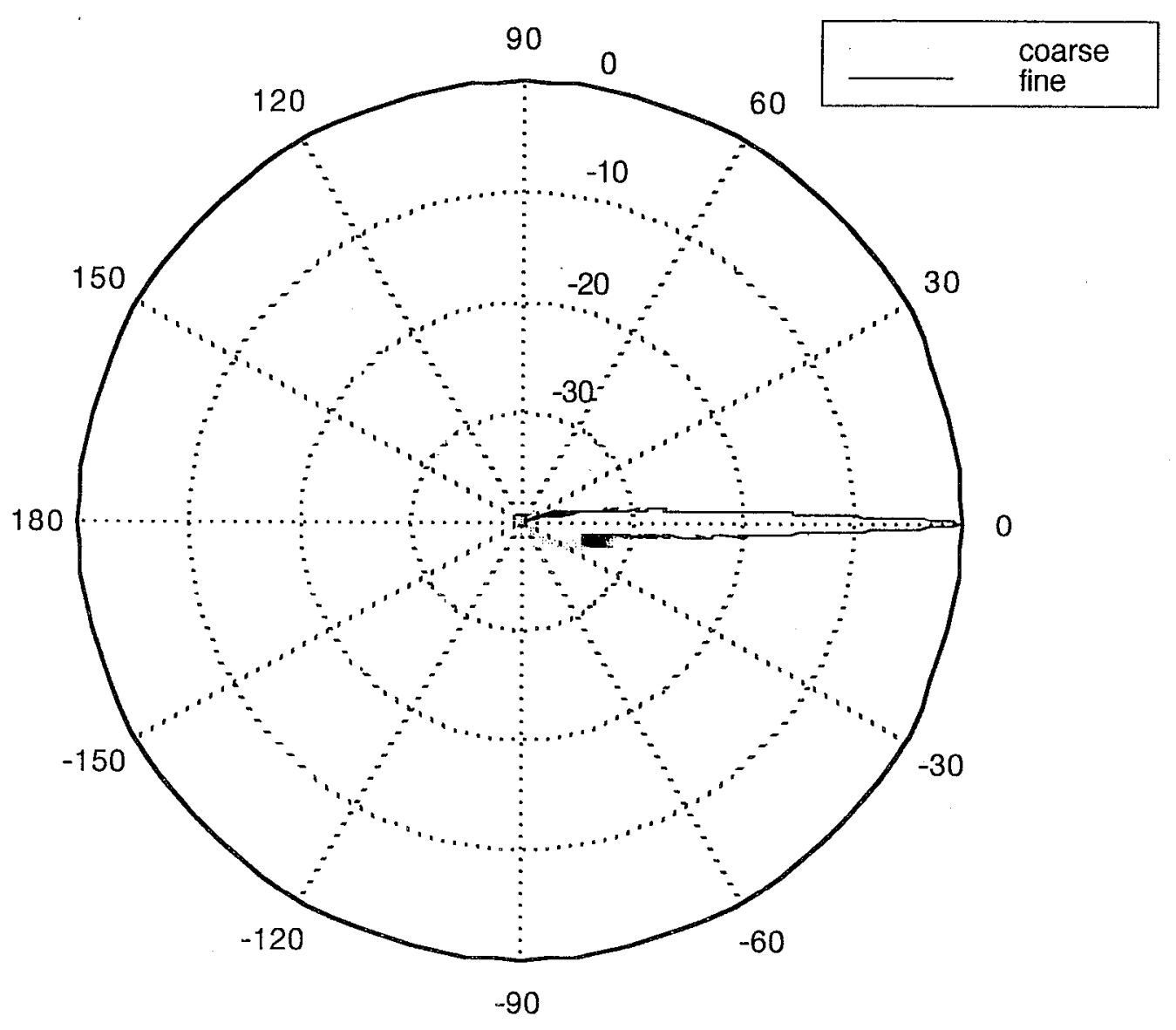




\section{Appendix E.1.d Stretched Beam Patterns}

Beam pattern for MPS1 stretched, $100 \mathrm{ft}$. range

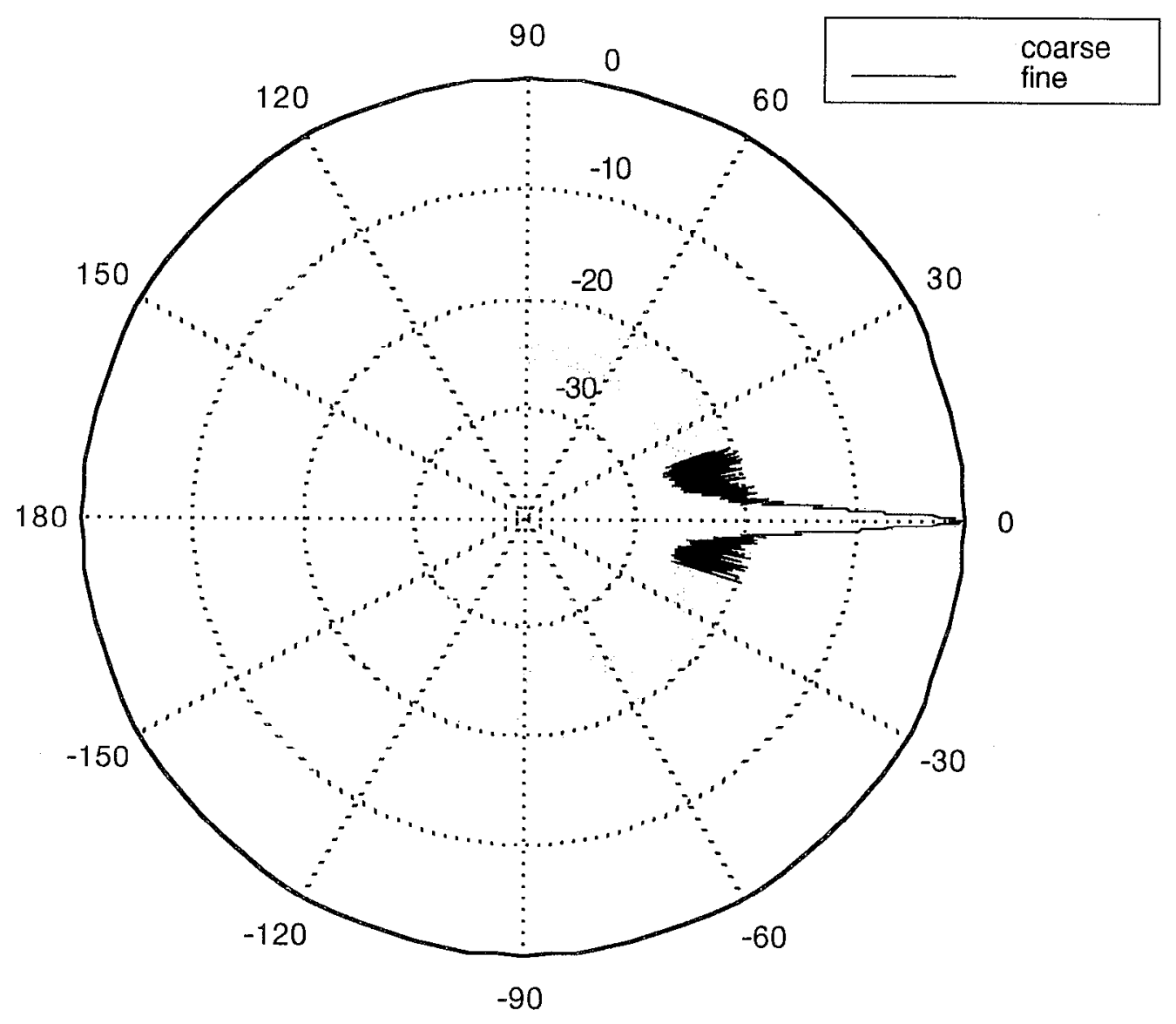


Beam pattern for MPS 1 compressed, $100 \mathrm{ft}$. range

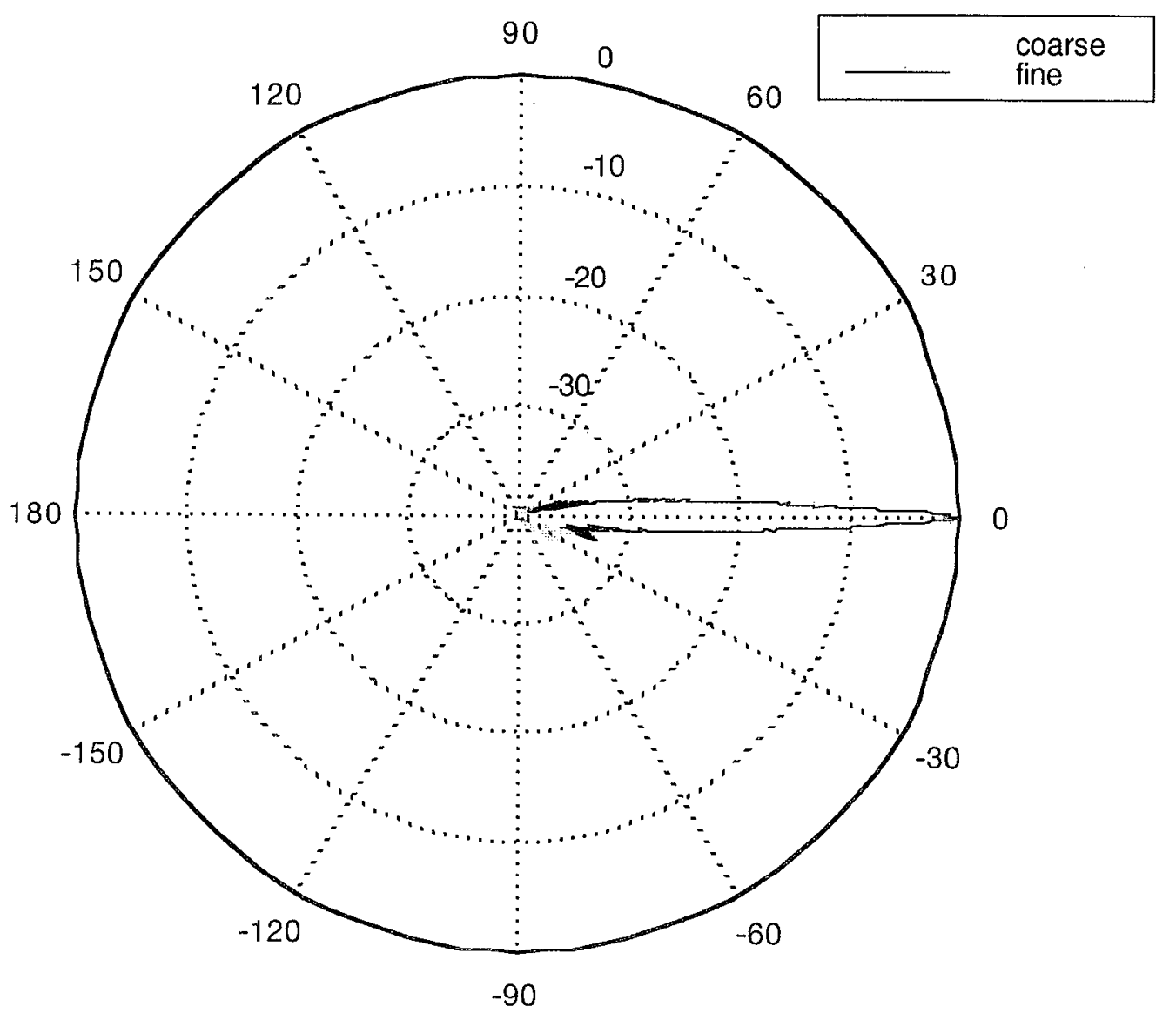


Beam pattern for MPS1 piston stretched, $100 \mathrm{ft}$. range

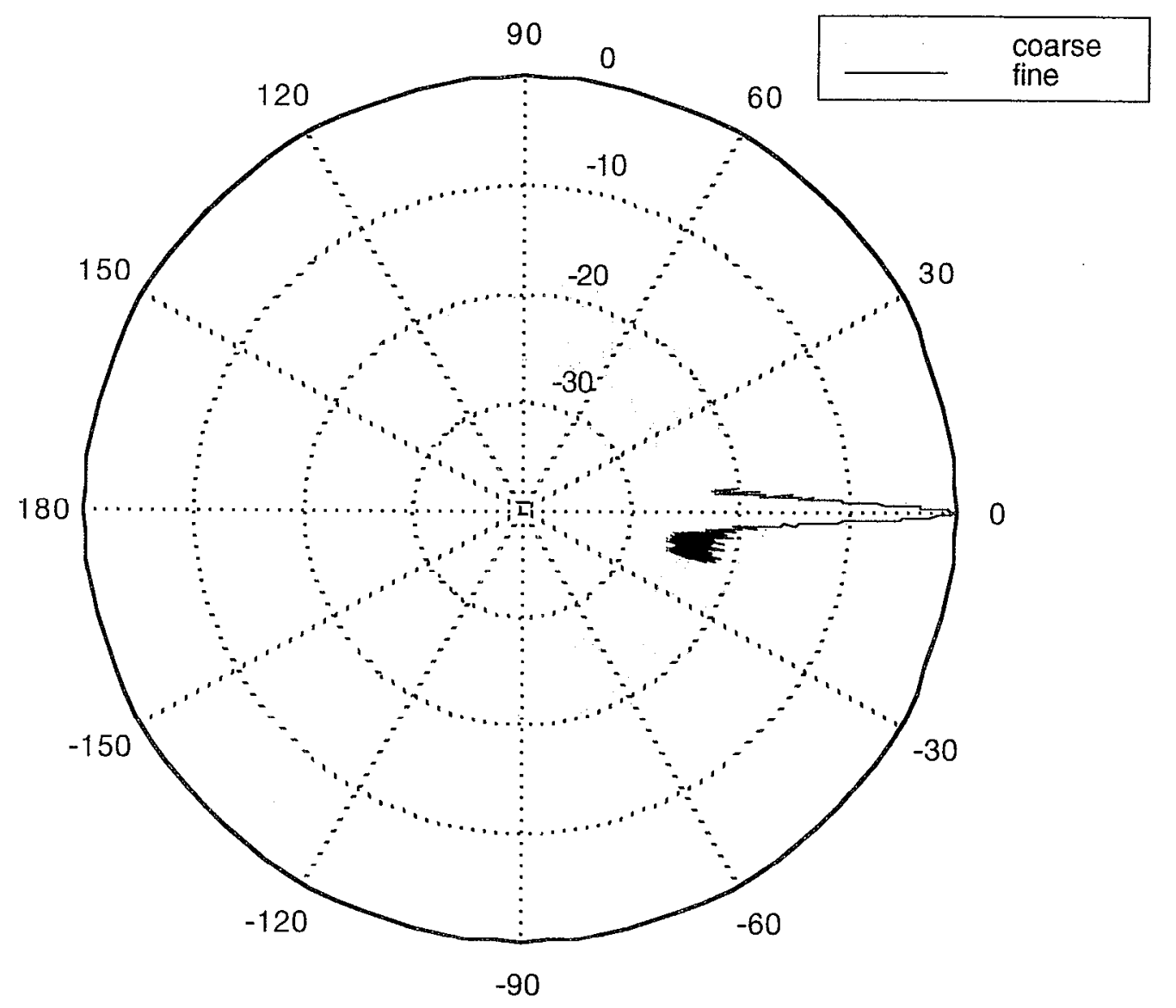


Beam pattern for MPS1 piston compressed, $100 \mathrm{ft}$. range

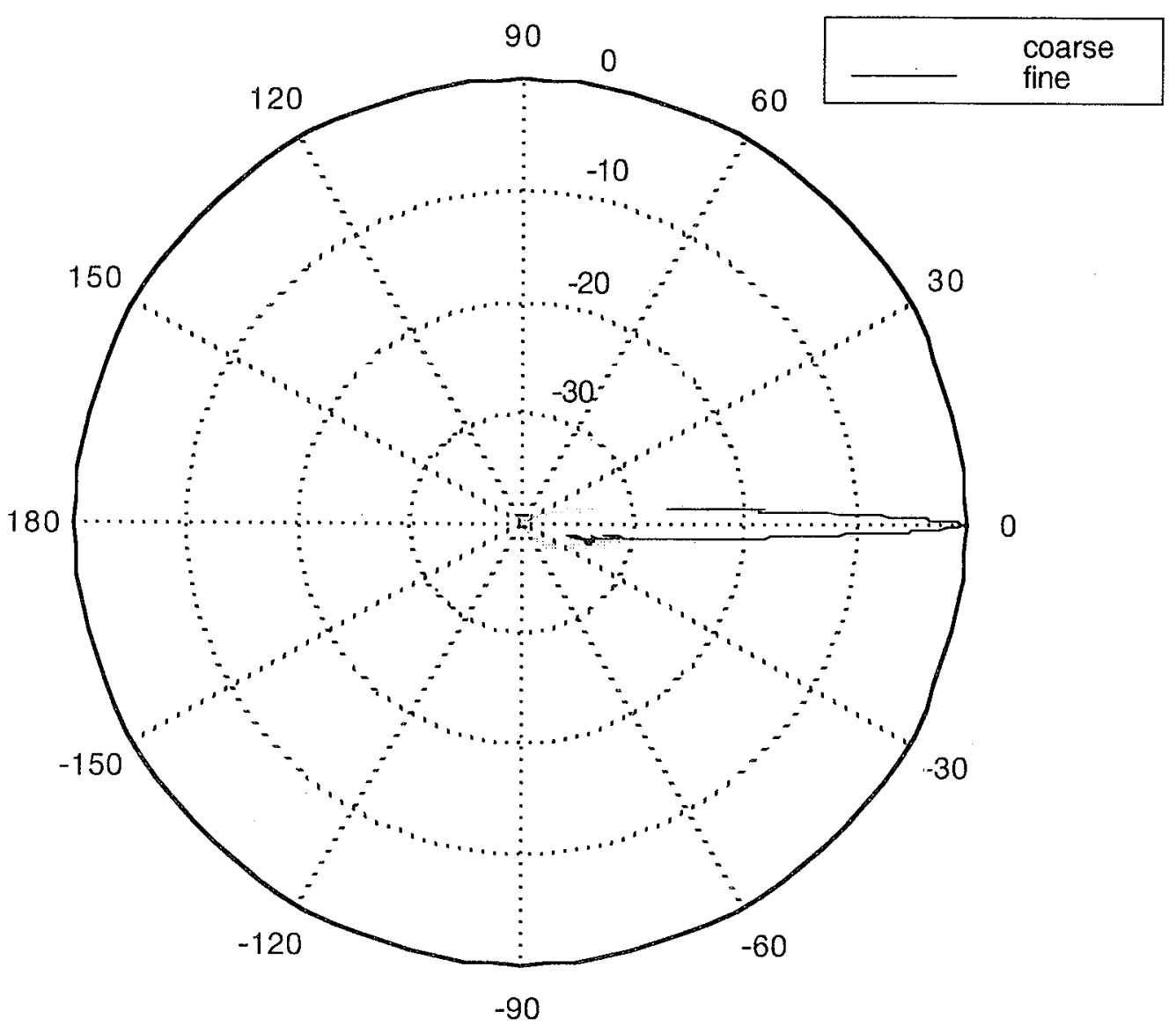


Appendix E.2 Polar energy plots, 600 foot range data

Appendix E.2.a 600 foot data, tone bursts.

Beam pattern for $20 \mathrm{kHz}$ tone burst, 200 yd. range

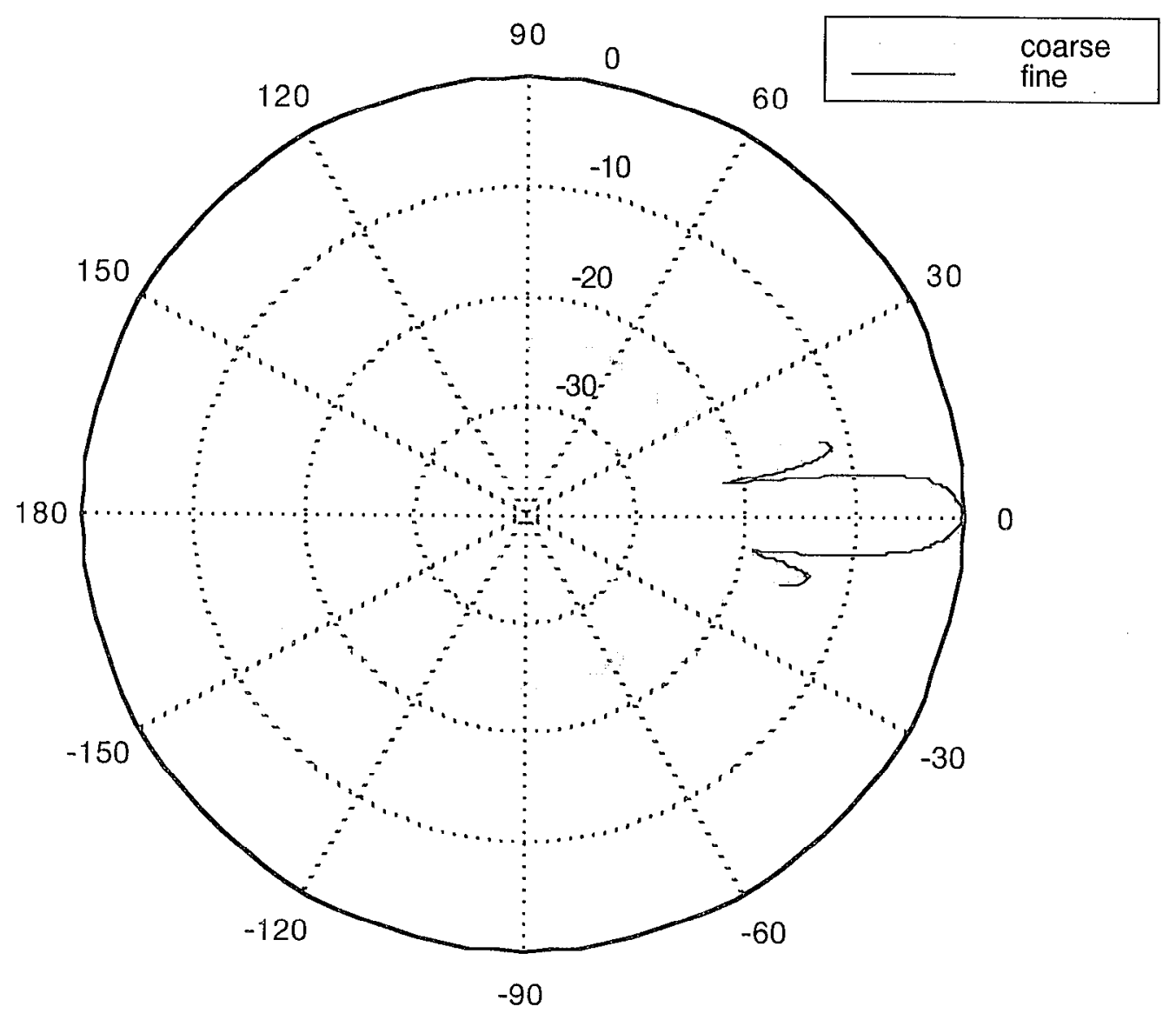


Beam pattern for $100 \mathrm{kHz}$ tone burst, $200 \mathrm{yd}$. range

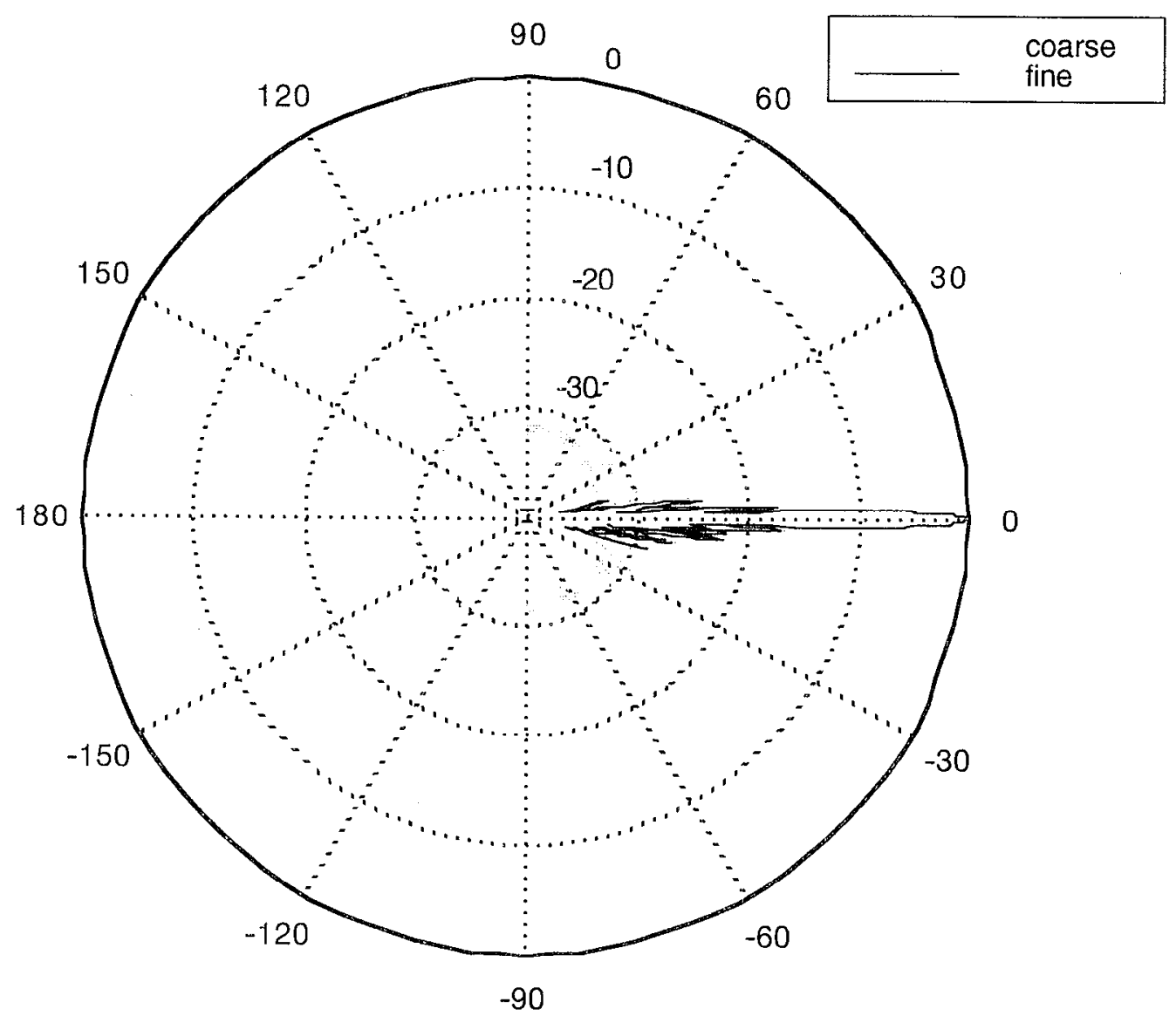


Beam pattern for $240 \mathrm{kHz}$ tone burst, 200 yd. range

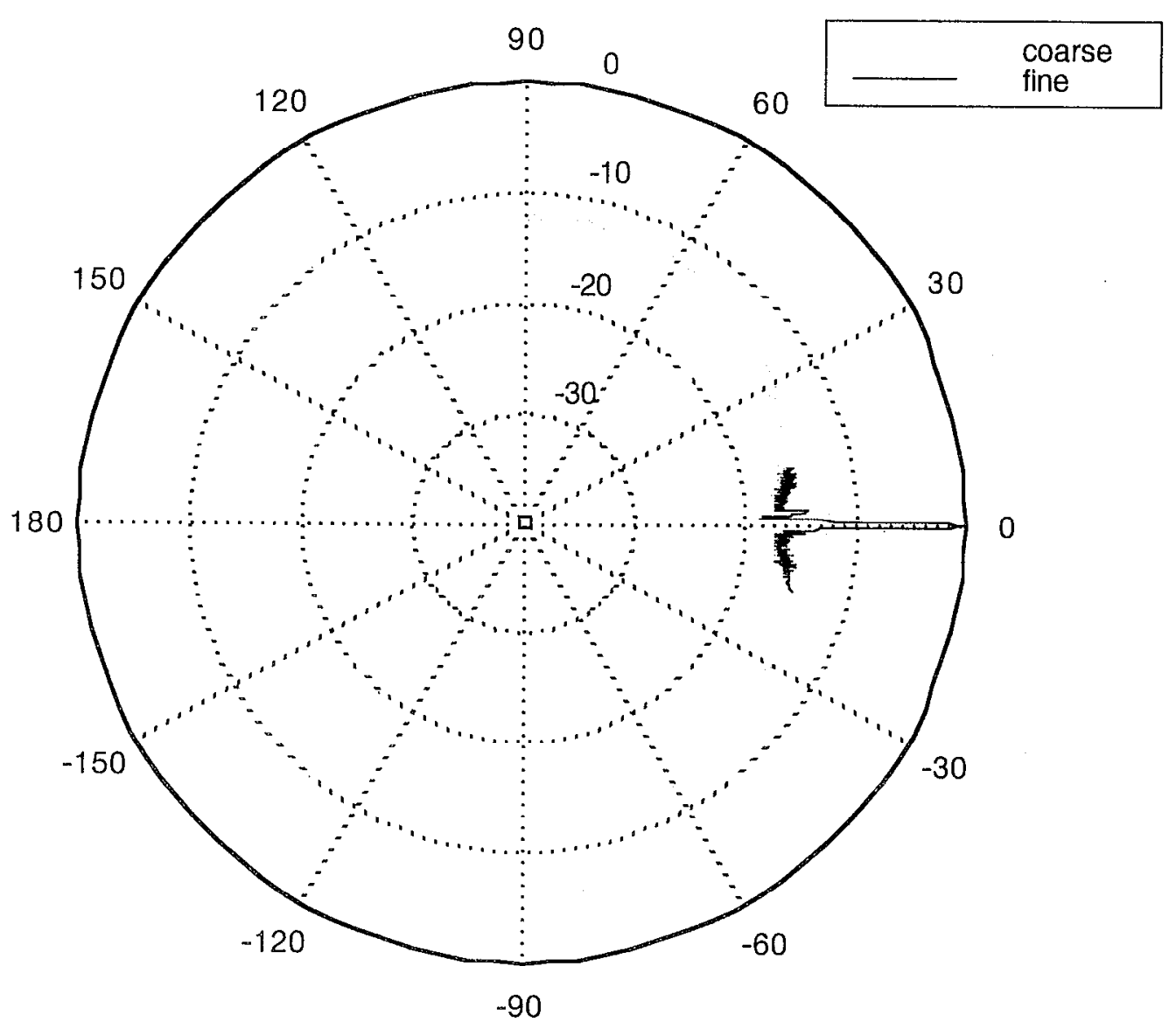




\section{Appendix E.2.b 600 foot data, Pulse data.}

Beam pattern for MPS1 2D, 200 yd. range

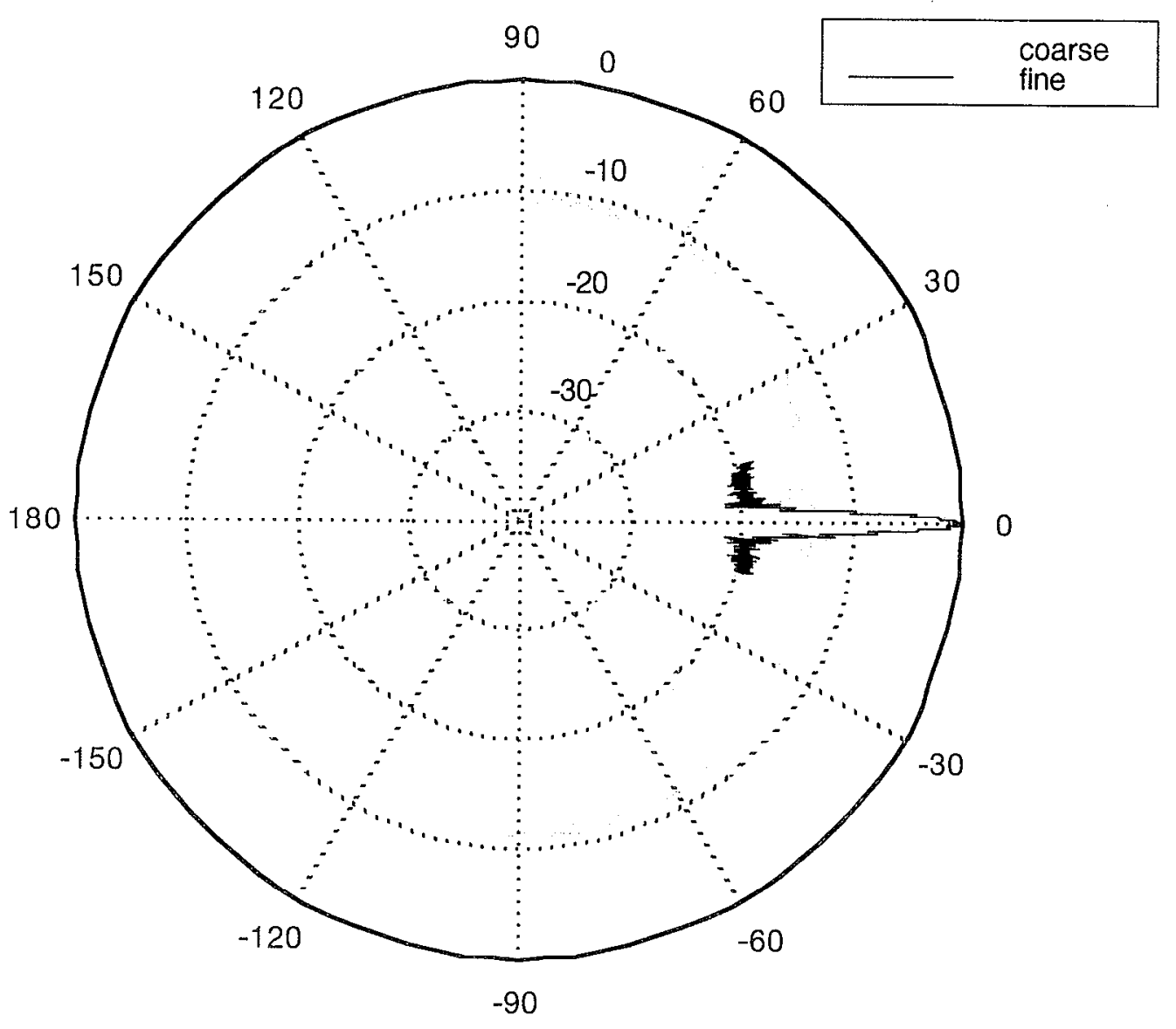




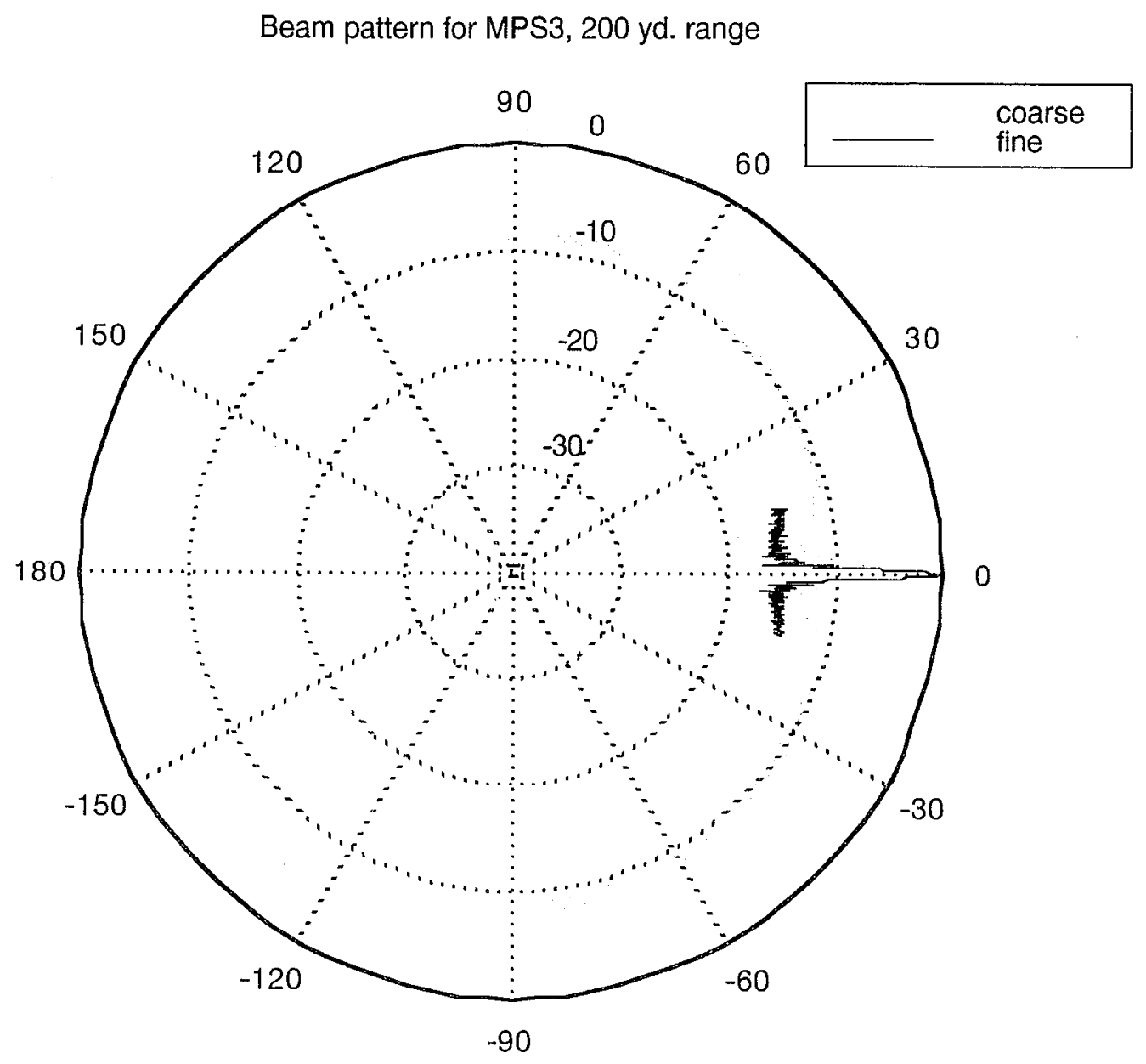




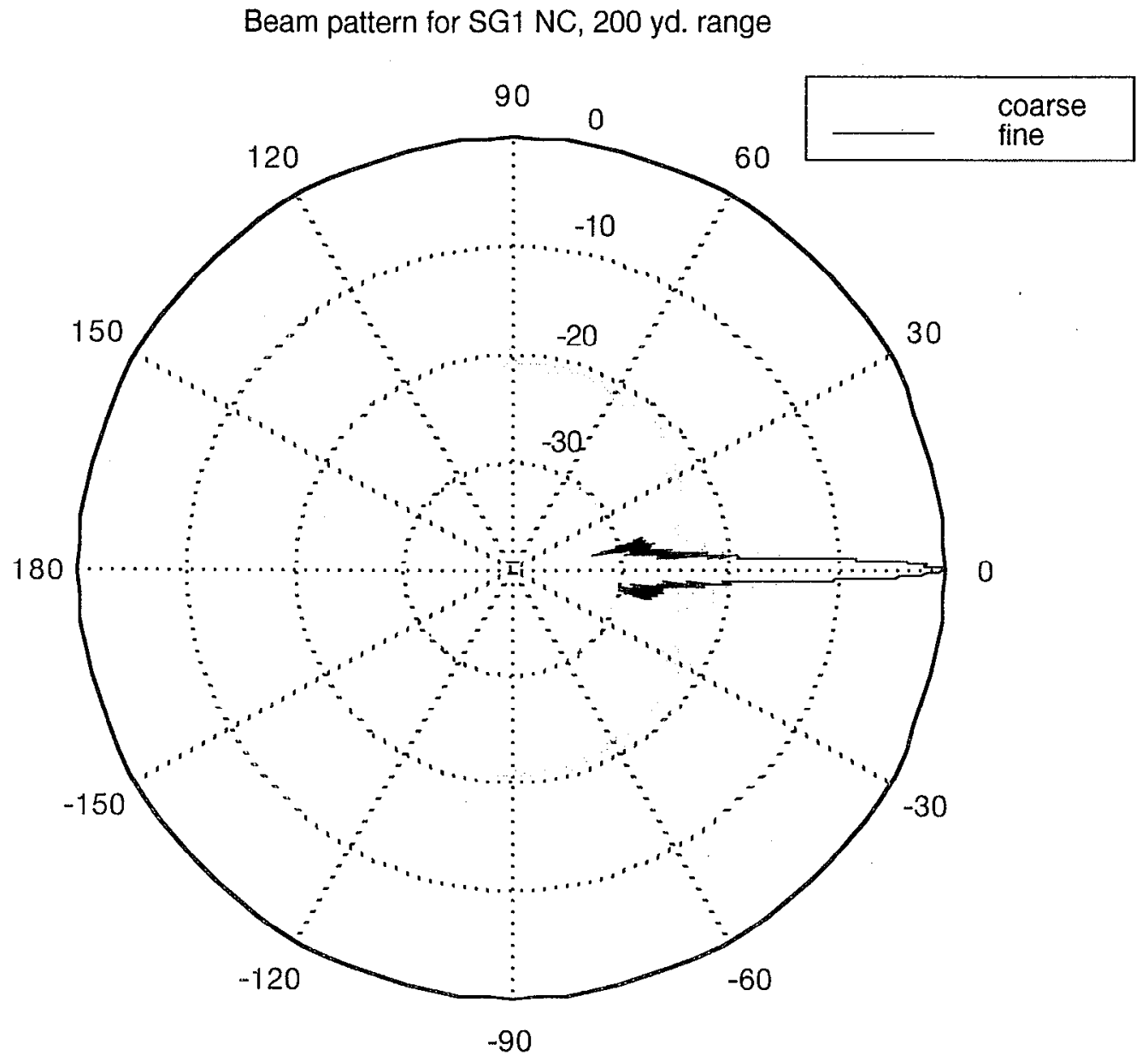




\section{Appendix E.2.c Piston pulse Beam Patterns}

Beam pattern for MPS1 2D piston, 200 yd. range

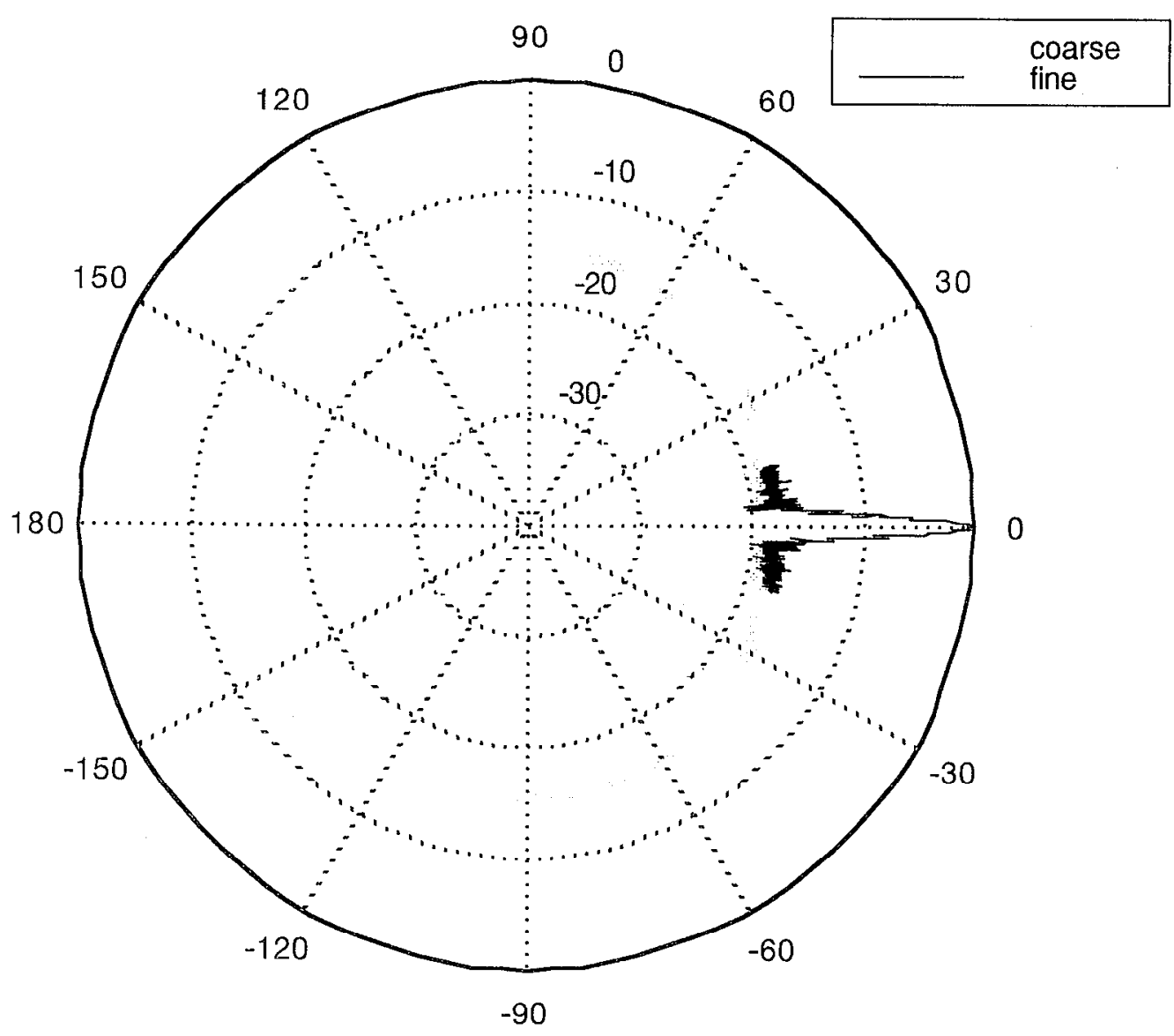


Beam pattern for MPS3 piston, 200 yd. range

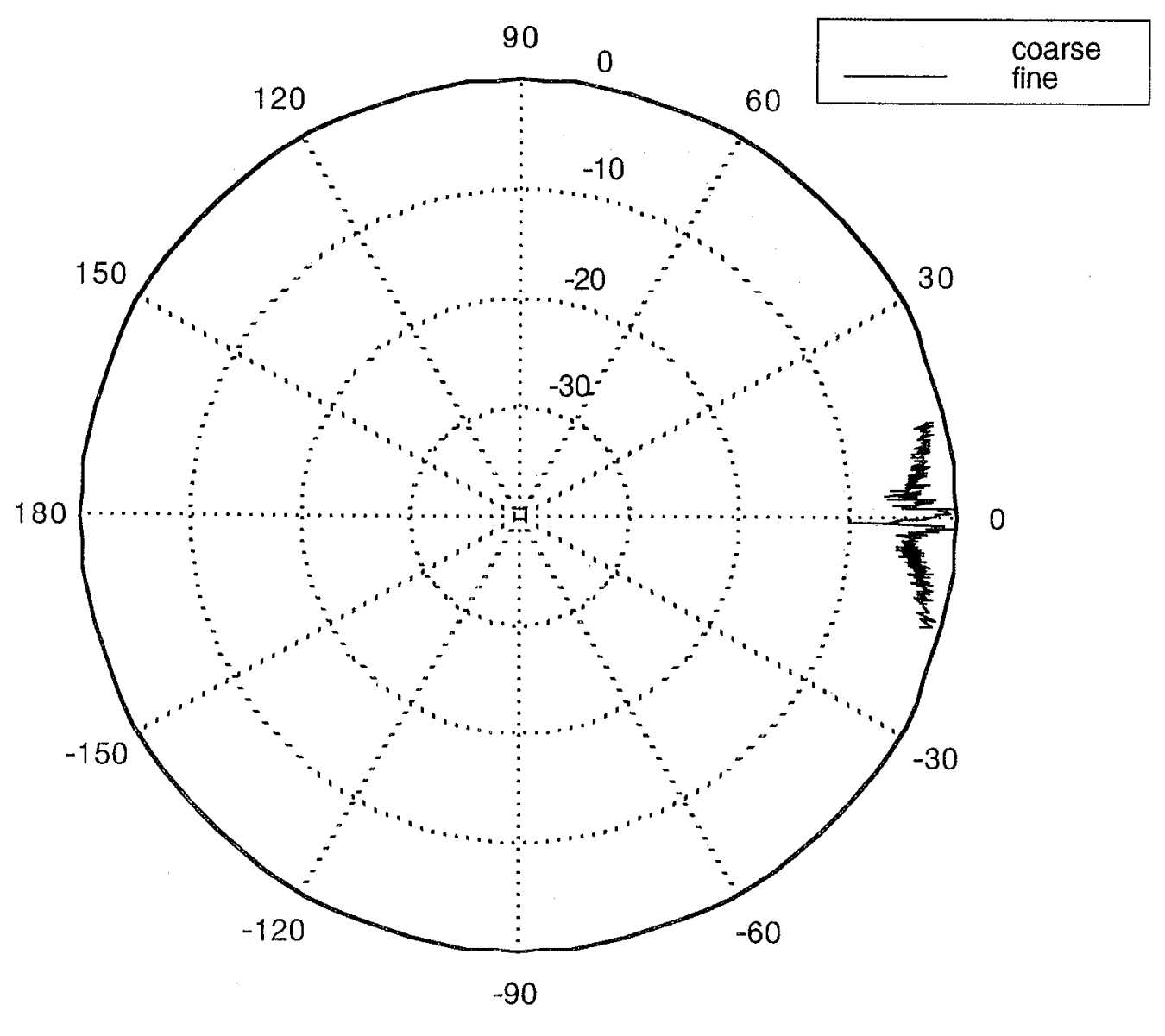

6 
Beam pattern for SG1 NC piston, 200 yd. range

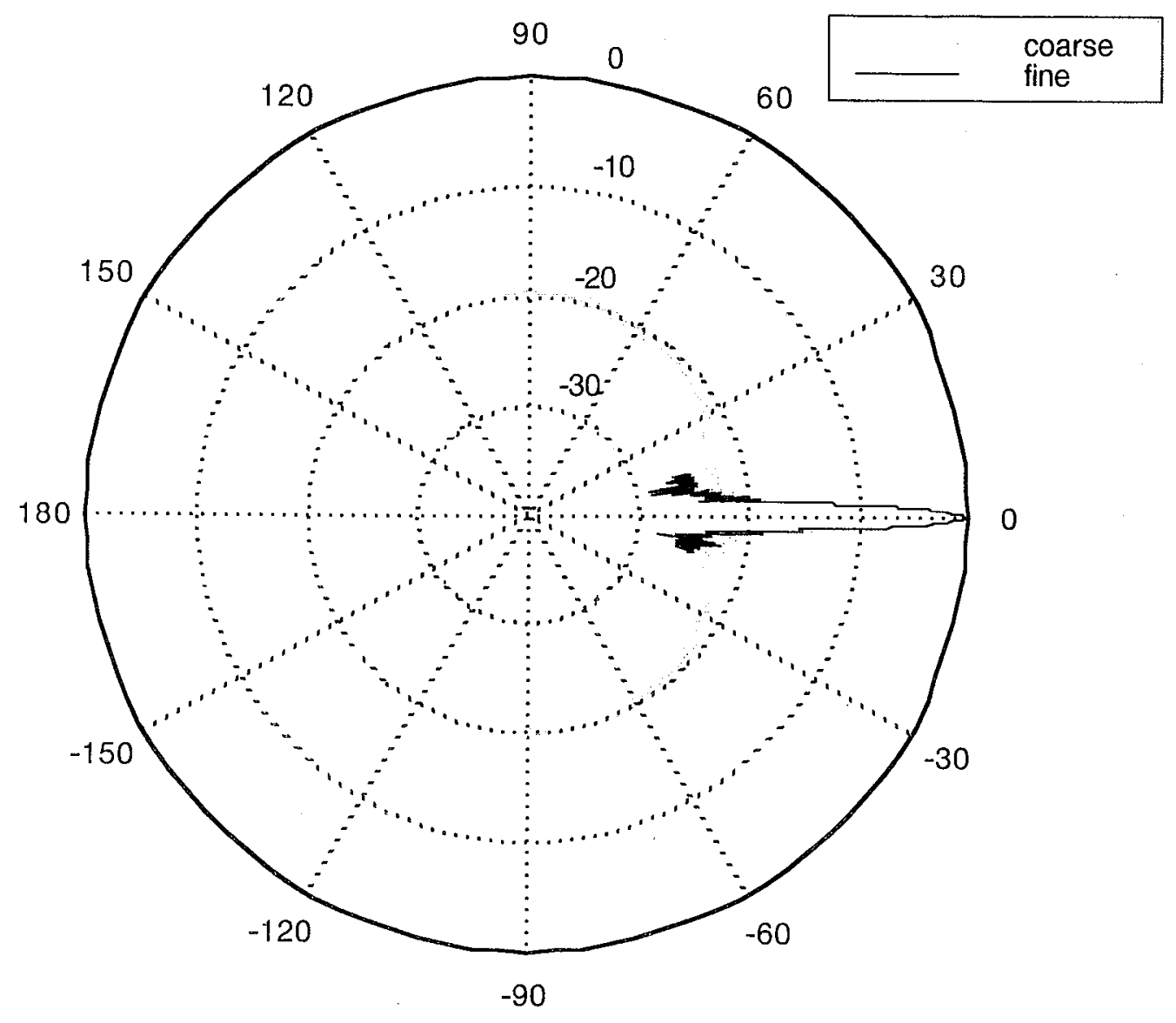




\section{Appendix E.2.d Stretched Beam Patterns}

Beam pattern for MPS1 stretched, $200 \mathrm{yd}$. range

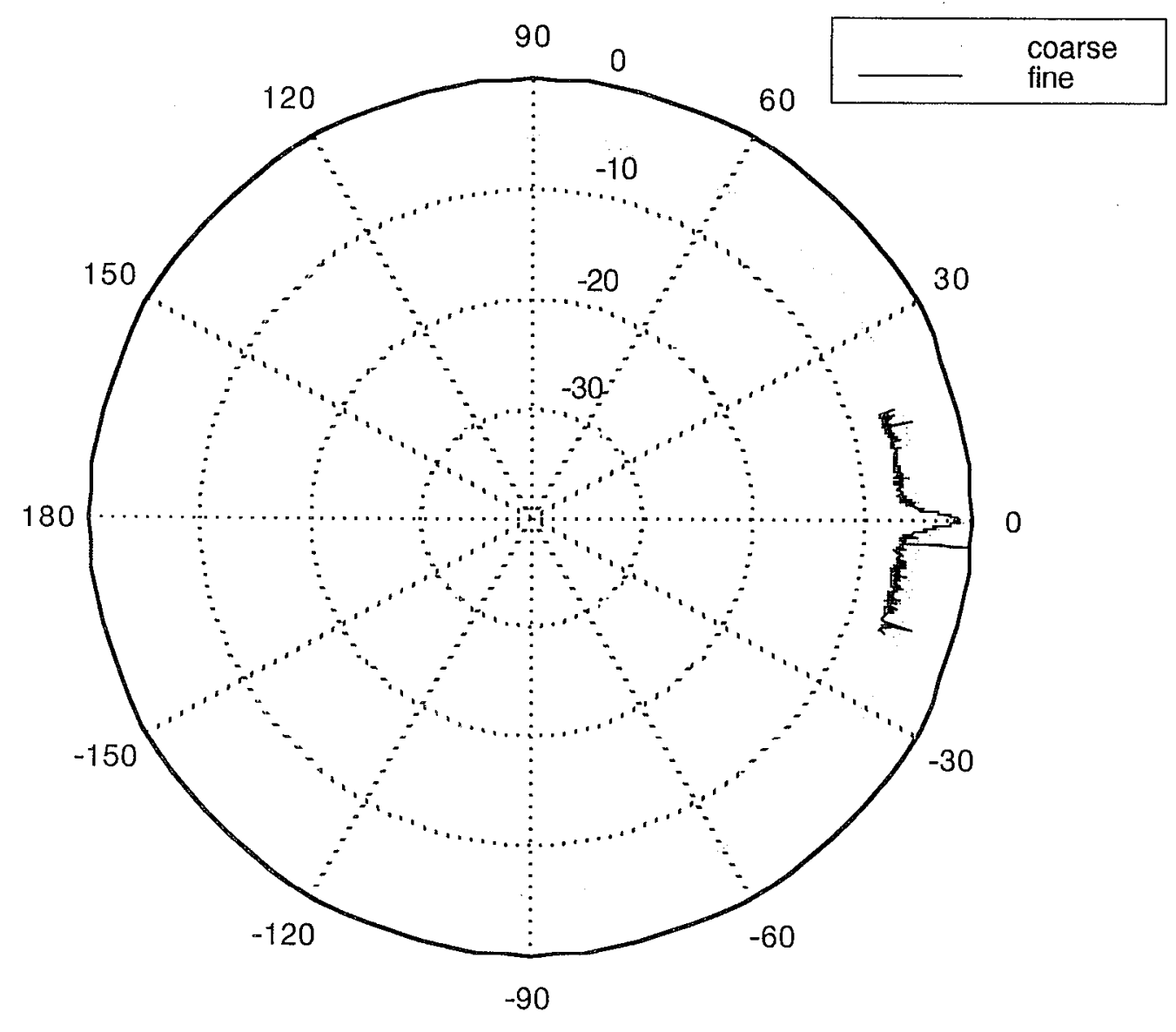


Beam pattern for MPS1 compressed, 200 yd. range

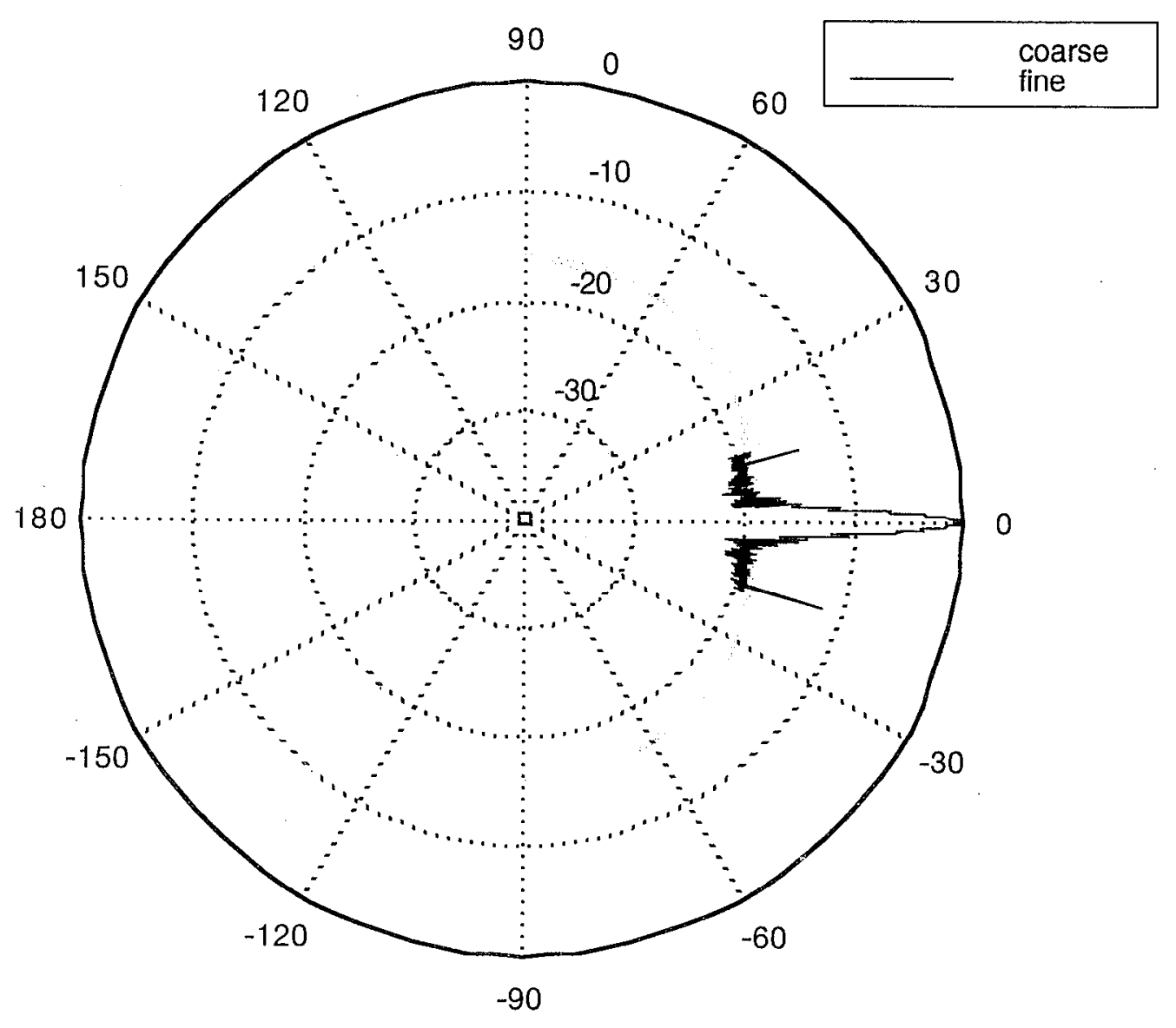


Beam pattern for MPS1 piston stretched, 200 yd. range

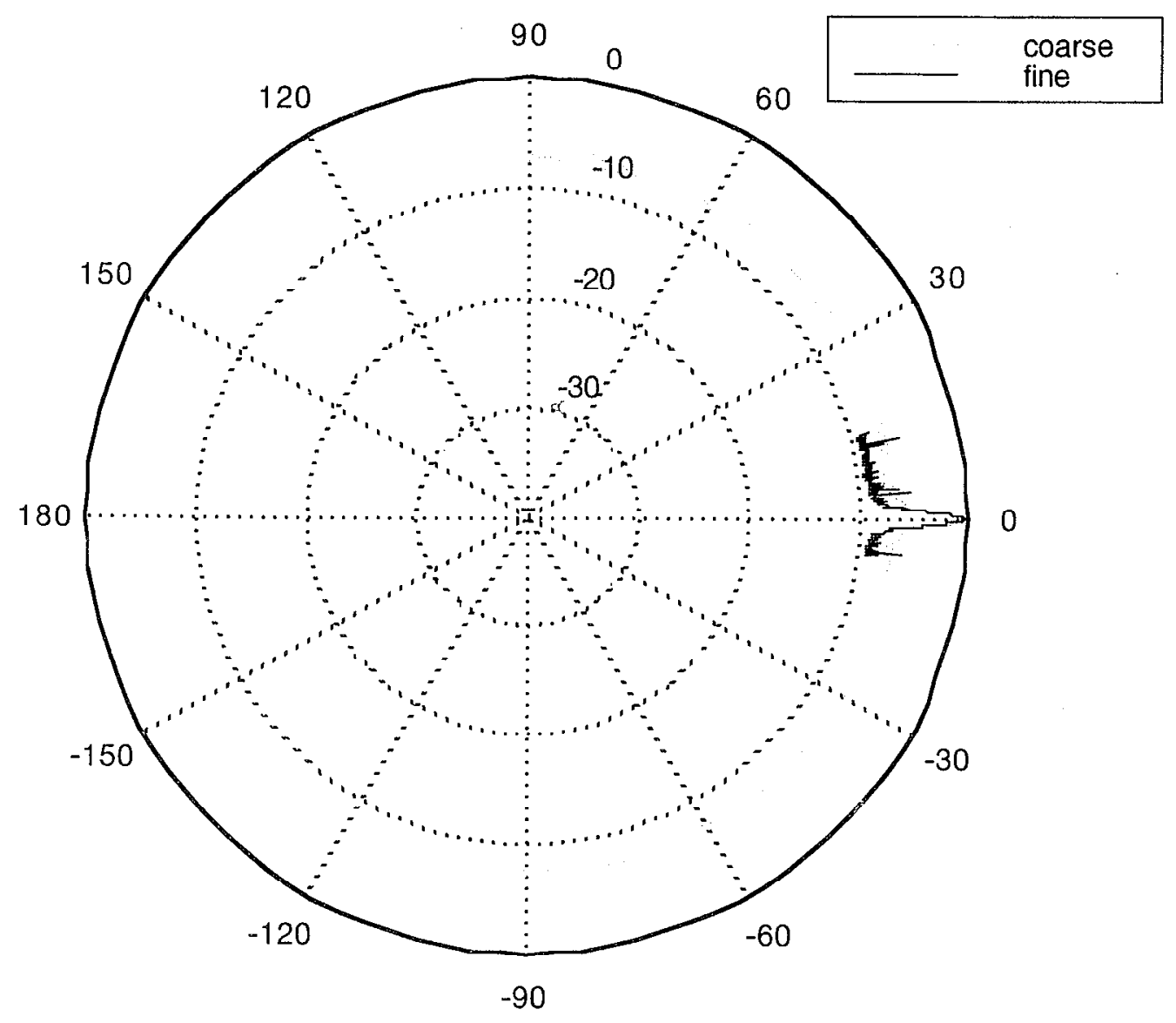


Beam pattern for MPS1 piston compressed, 200 yd. range

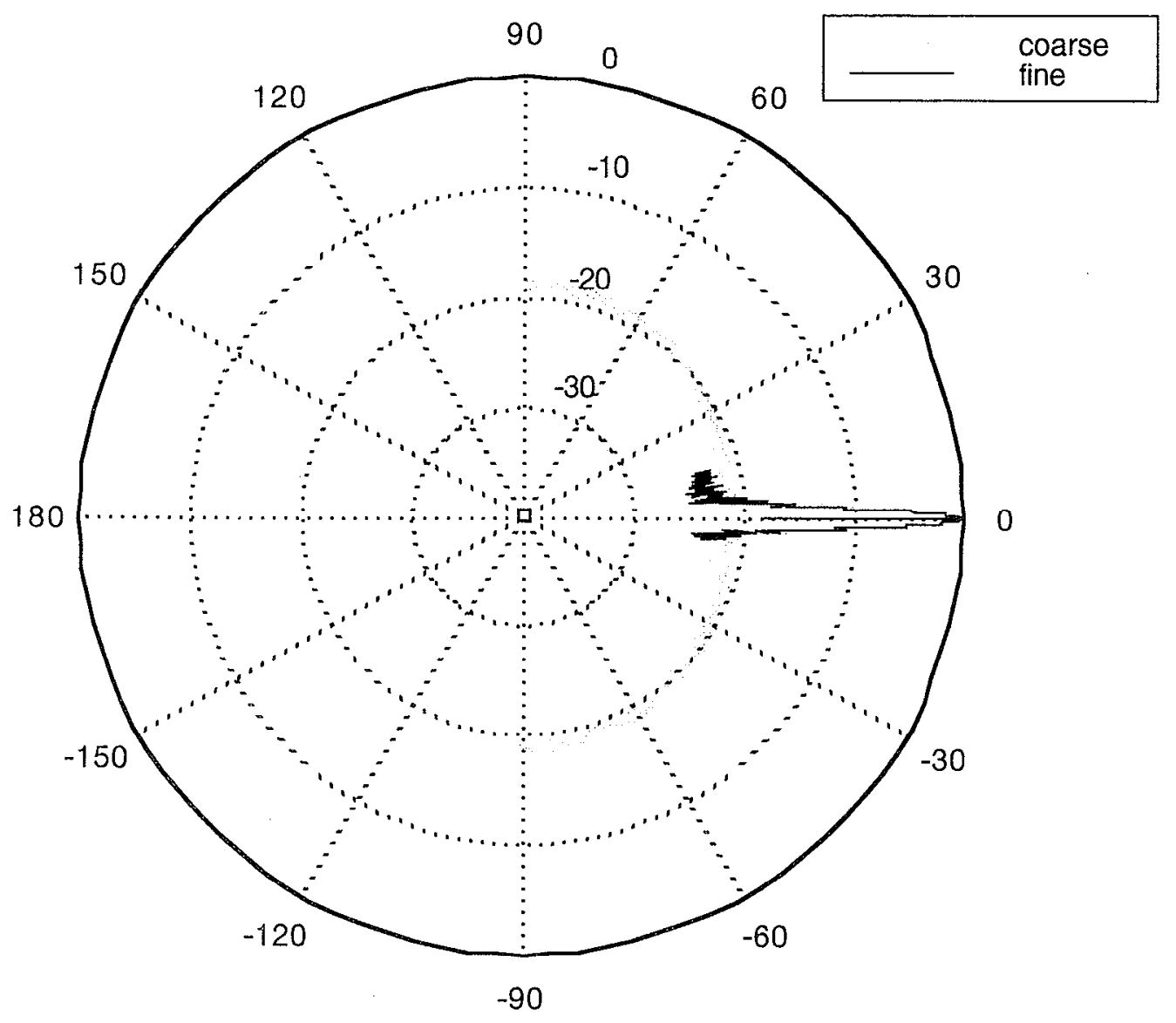


Beam pattern for MPS3 stretched, 200 yd. range

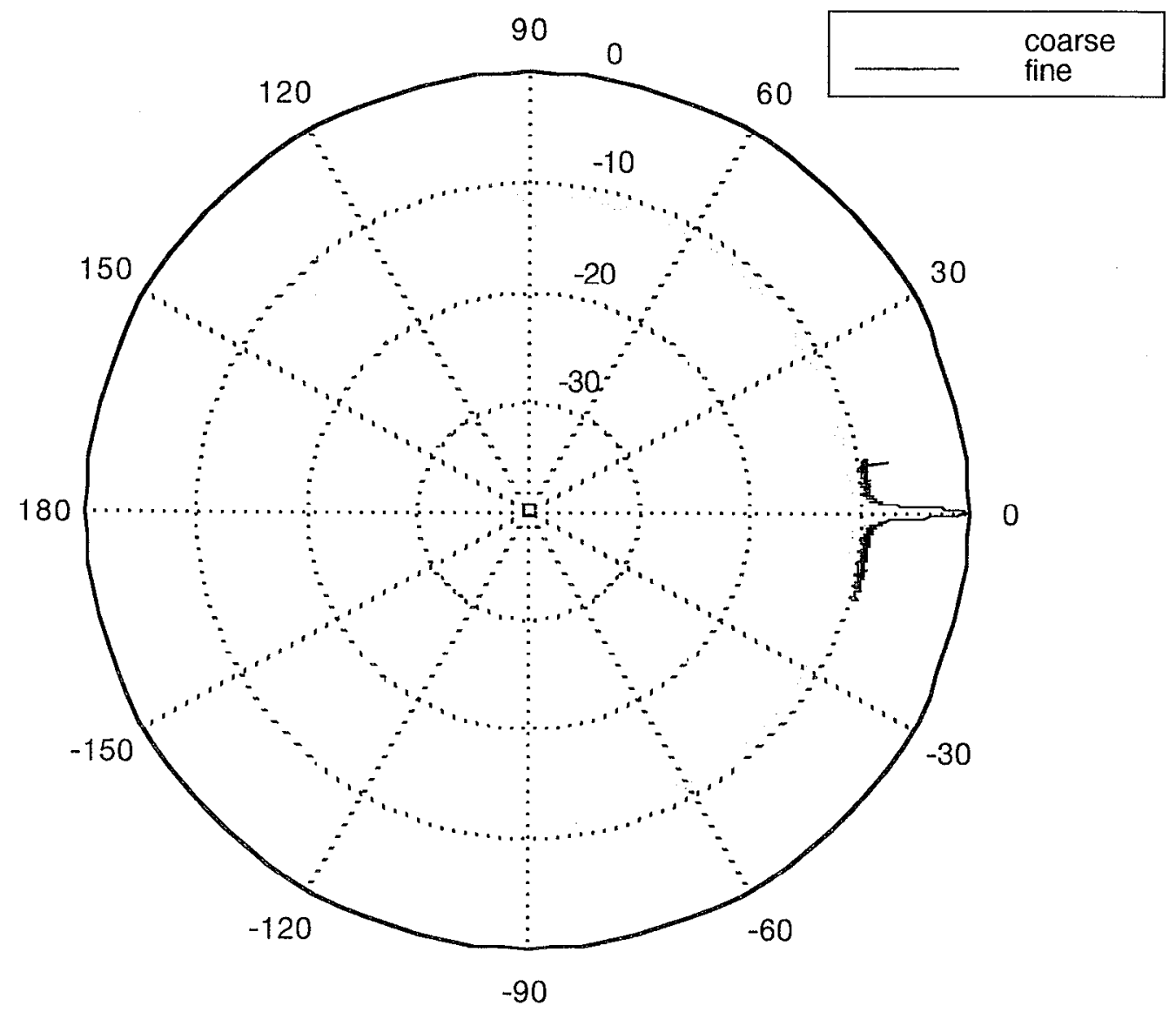


Beam pattern for MPS3 compressed, 200 yd. range

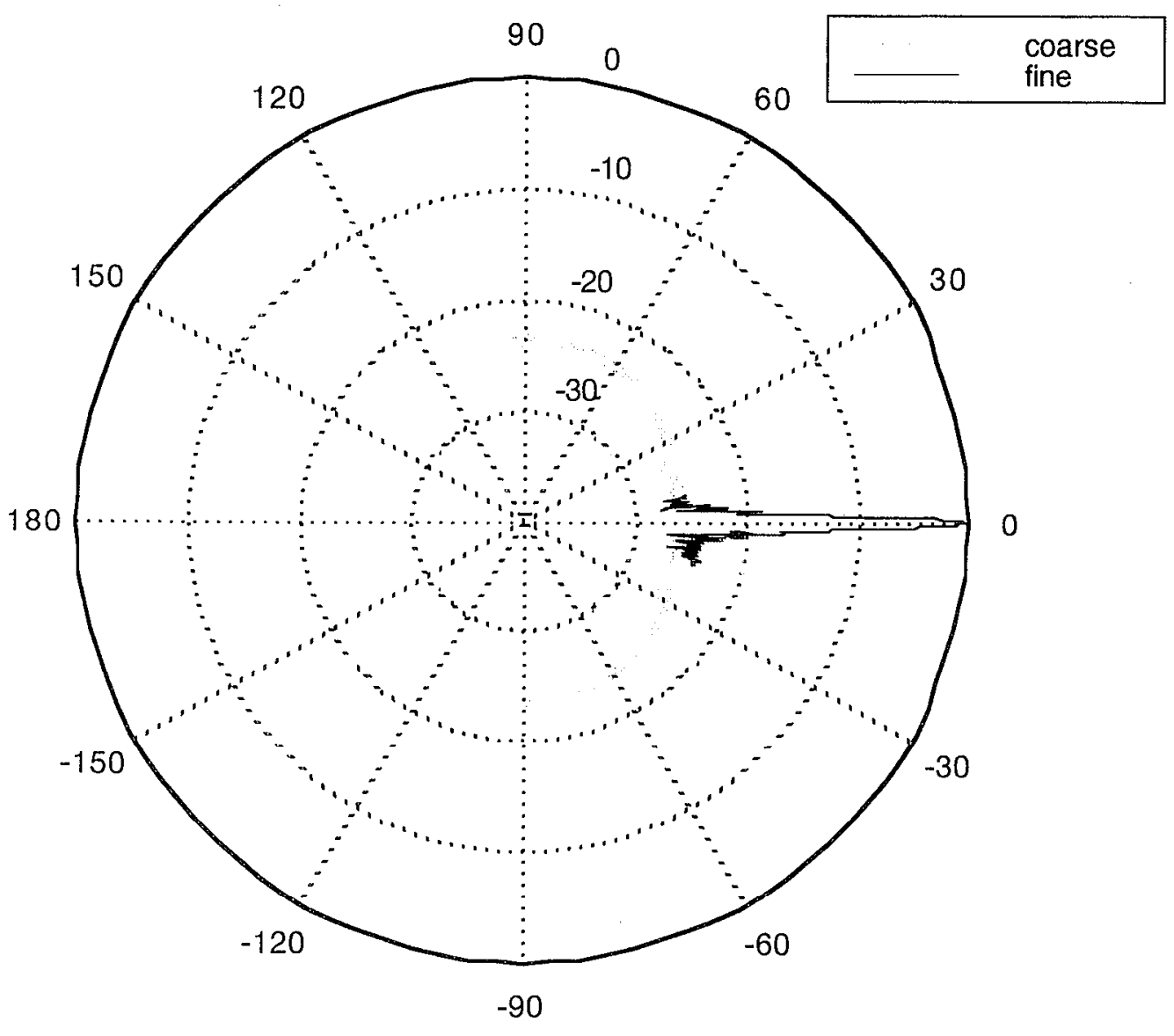




\section{Beam pattern for MPS3 piston stretched, $200 \mathrm{yd}$. range}

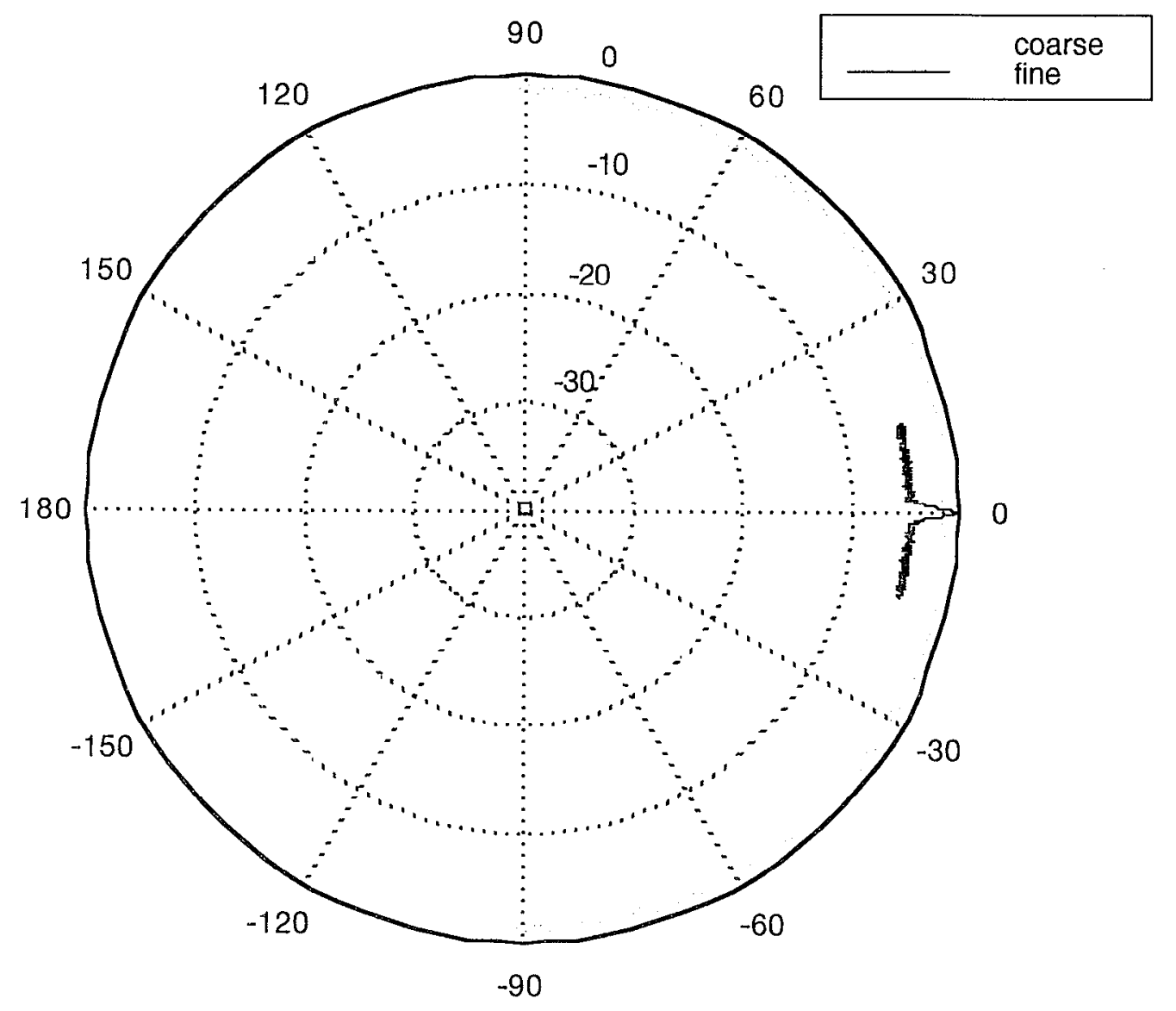


Beam pattern for MPS3 piston compressed, $200 \mathrm{yd}$. range

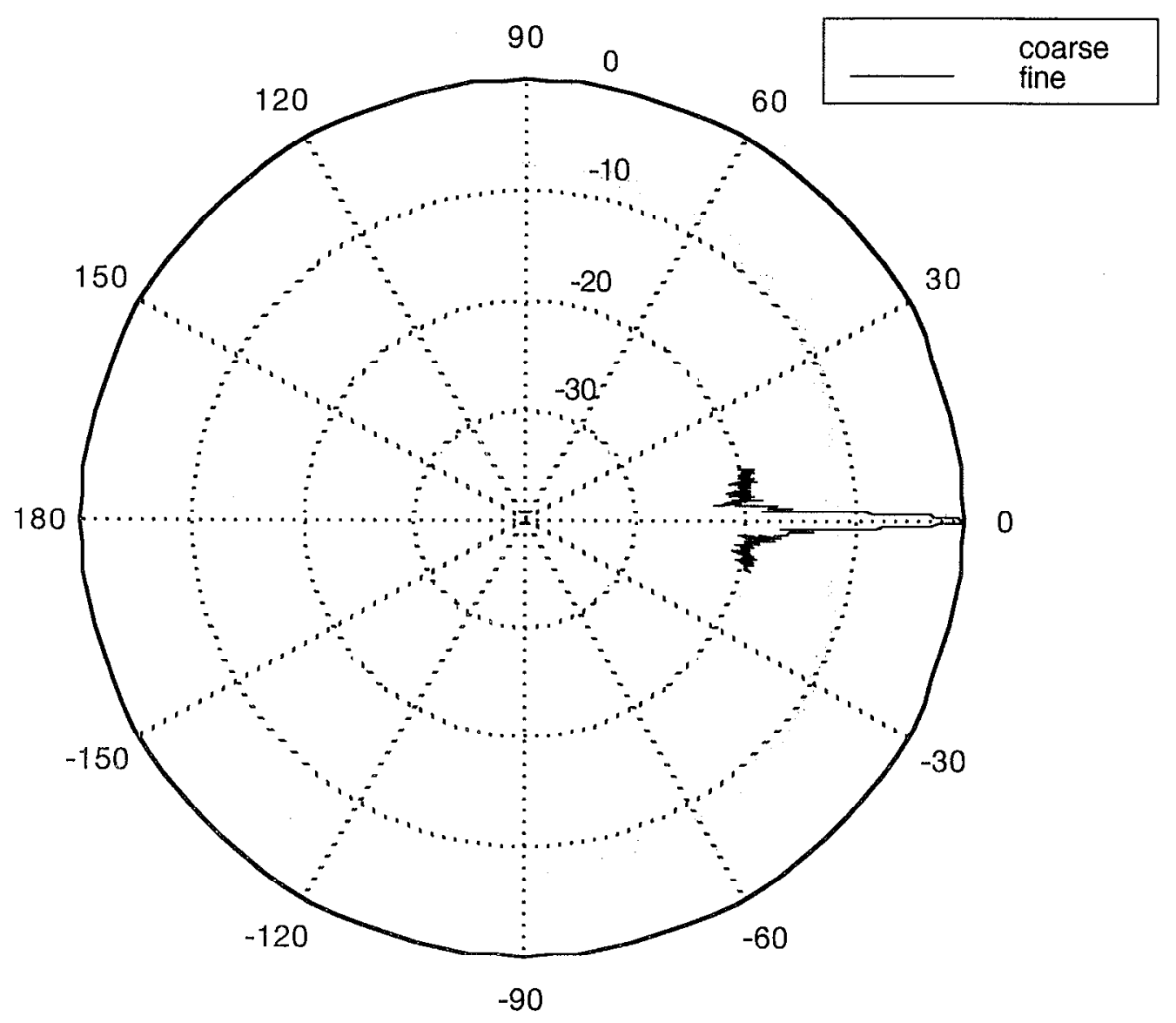




\section{Appendix F. Source Signals}

The following is a catalog of the source signals with their associated frequency spectra. The three pulses used, MPS1, SG1 and MPS3, are shown as generated, and in some cases with compensation to make the received signal resemble either the second (2D) or third (3D) derivative of the input center signal.

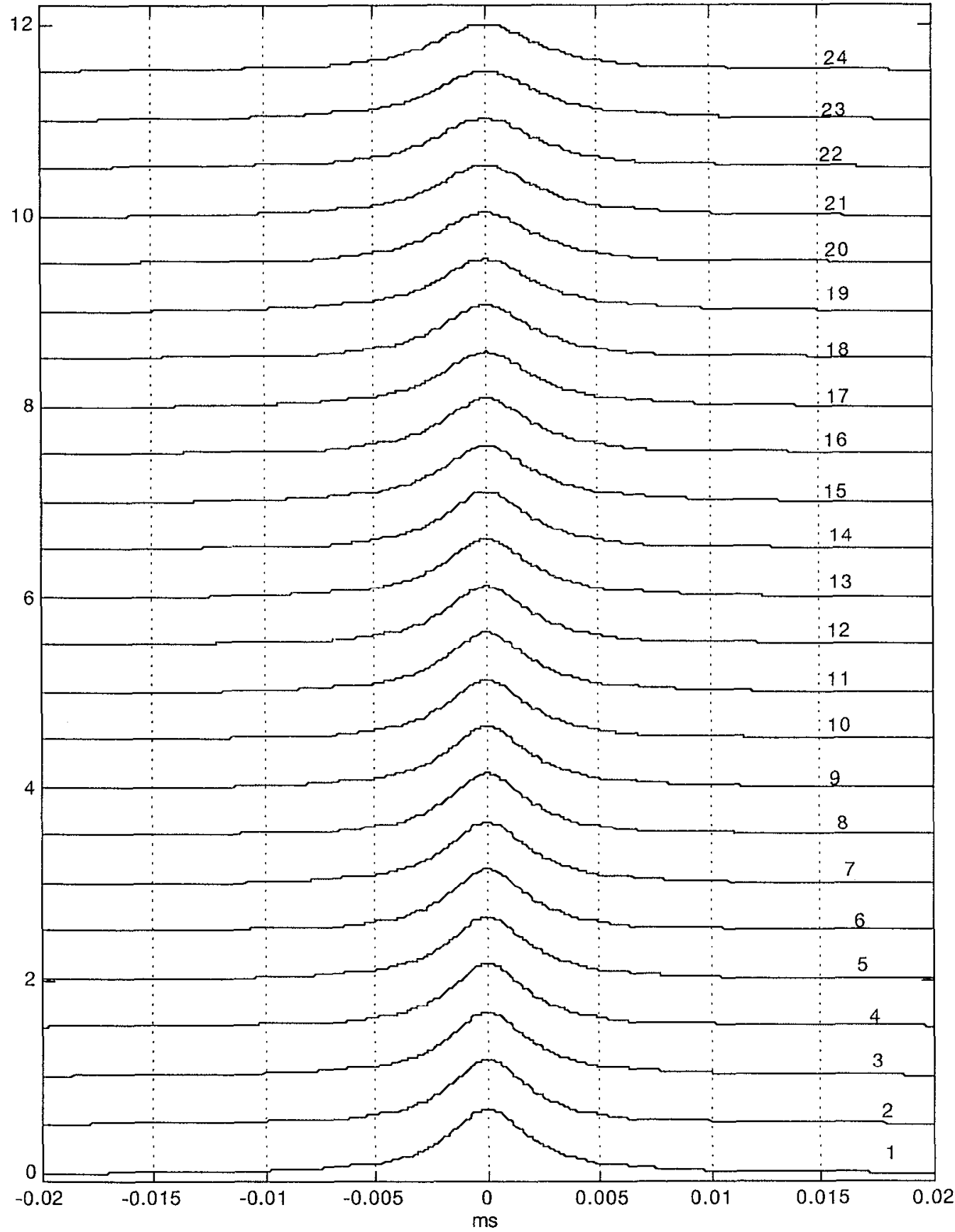

Figure F.1.a The input source signal time traces for MPSI pulse with no frequency compensation. The base line of each signal has been shifted for clarity, and all actually start at 0 volts. The vertical axis in in arbitrary units of volts, and the horizontal axis is in units of time with zero chosen at the source center. 
The spectra are simply the Fourier transform of the input signals, and their amplitude at zero frequency has been adjusted to $0 \mathrm{~dB}$ in all cases but the MPS 3 case. The shape of the received signal is expected to change due to diffirentiation of the input signals by the array elements.

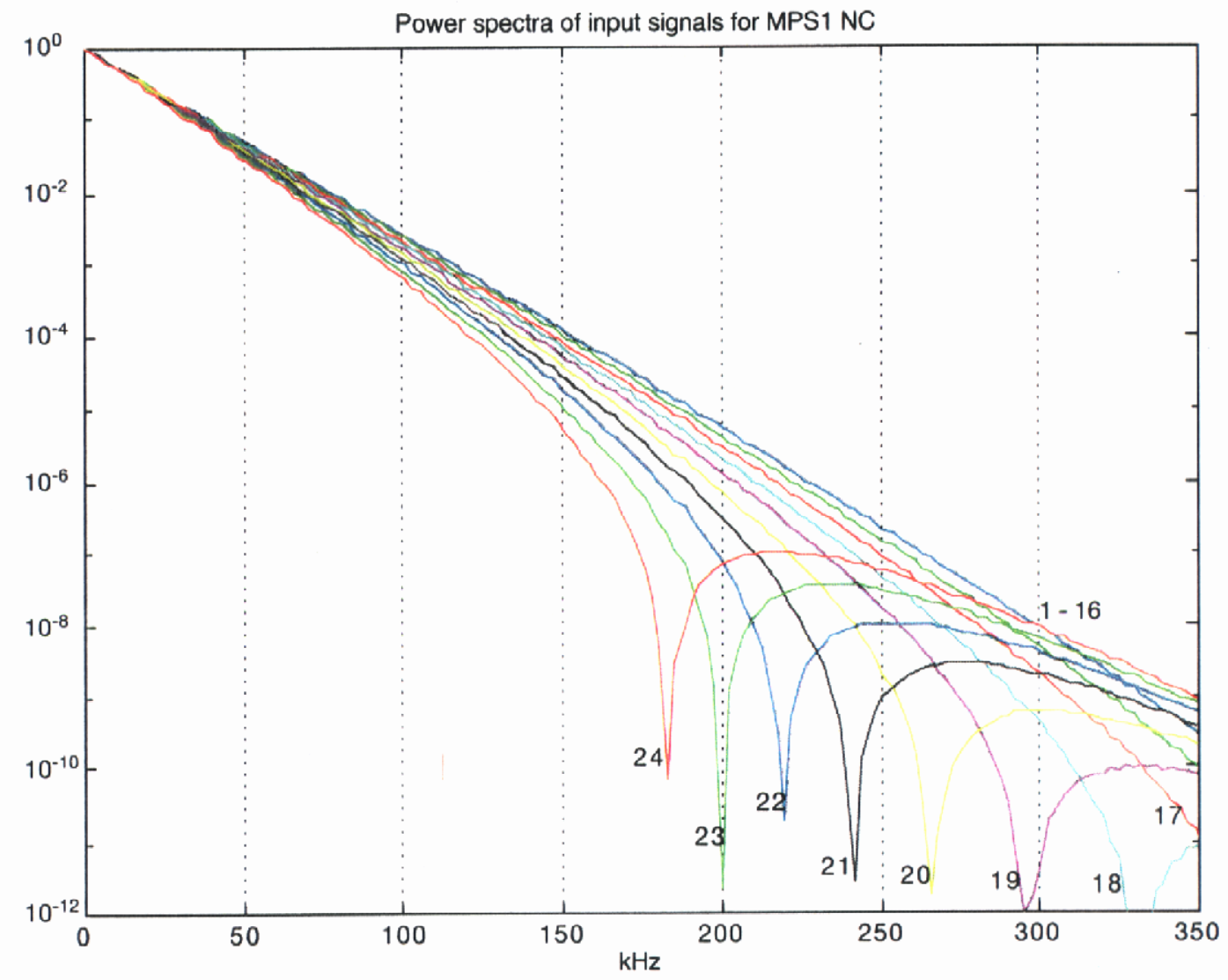

Figure F.1.b The spectra of the MPS1 pulse with no frequency compensation. The vertical axis is in arbitrary units of amplitude on a log scale (x10 is $10 \mathrm{~dB}$ ), and the horizontal axis is in $\mathrm{kHz}$, from 0 to $350 \mathrm{kHz}$ on a linear scale. 


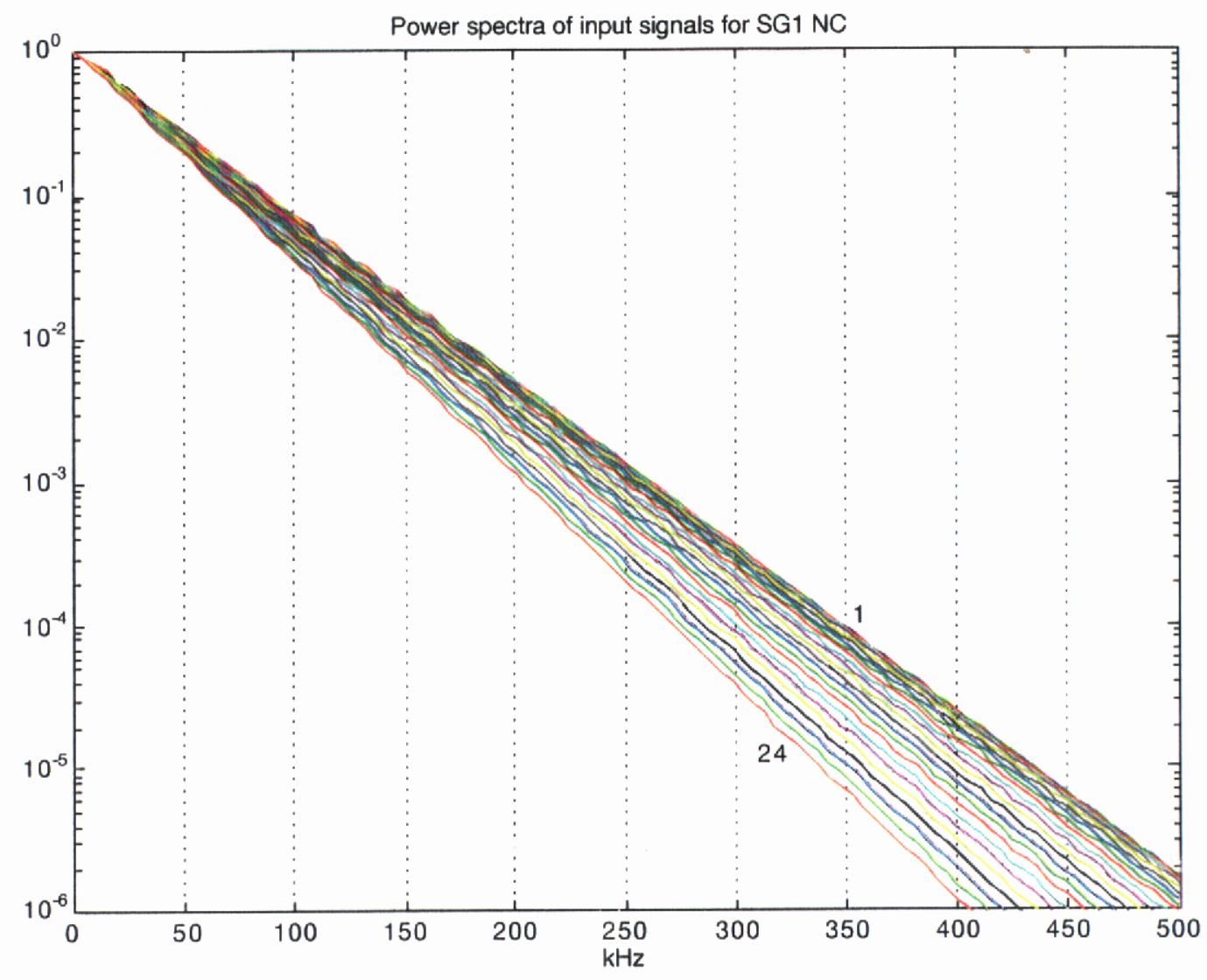

Figure F.4.b The spectra of the SG1 pulse with no frequency compensation. The vertical axis is in arbitrary units of amplitude on a log scale (x10 is $10 \mathrm{~dB})$, and the horizontal axis is in $\mathrm{kHz}$, from 0 to $500 \mathrm{kHz}$ on a linear scale. 


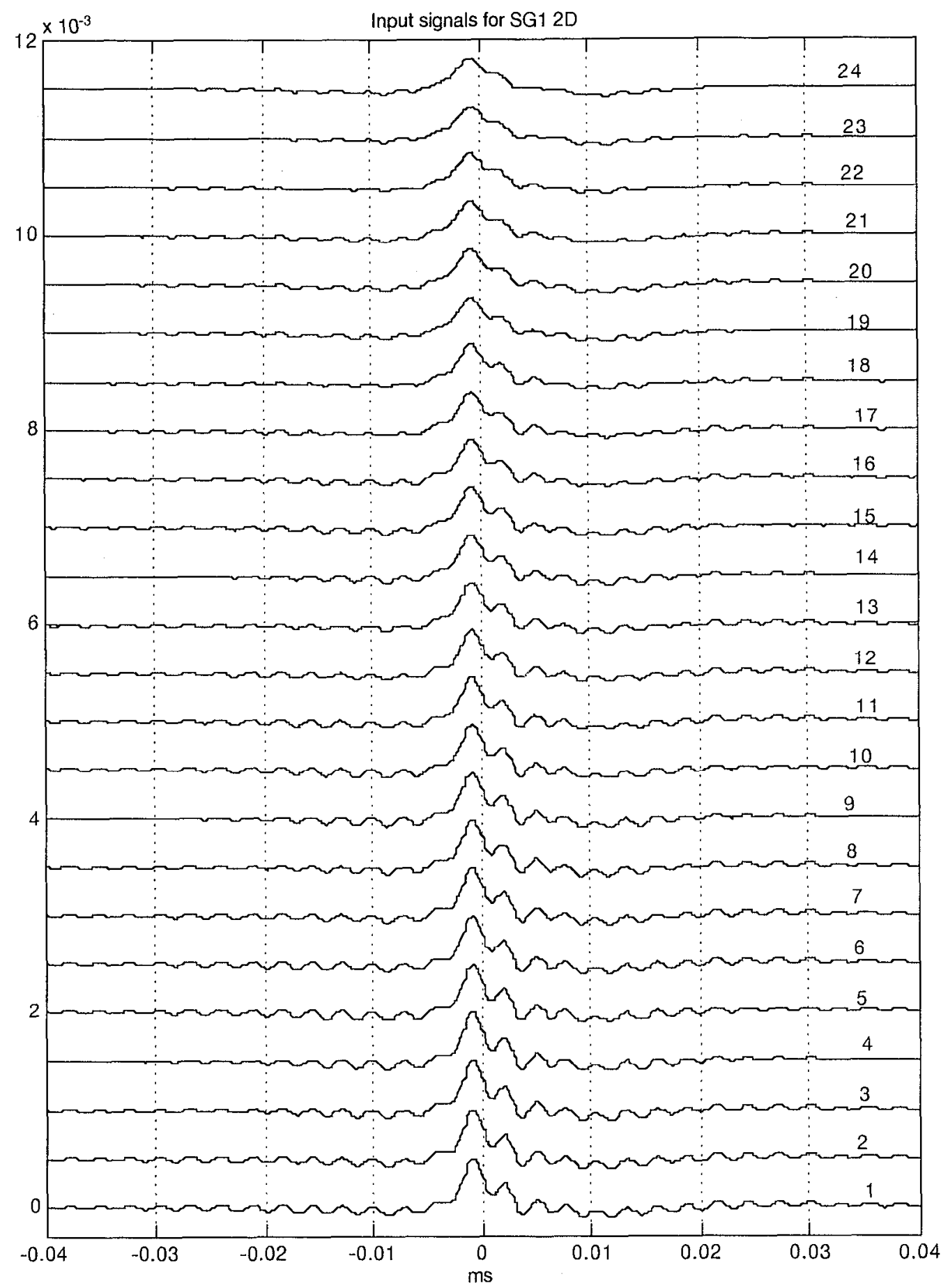

Figure F.5.a The input source signal time traces for SG1 pulse with 2D frequency compensation. The base line of each signal has been shifted for clarity, and all actually start at 0 volts. The vertical axis in in arbitrary units of volts, and the horizontal axis is in units of time with zero chosen at the source center. 


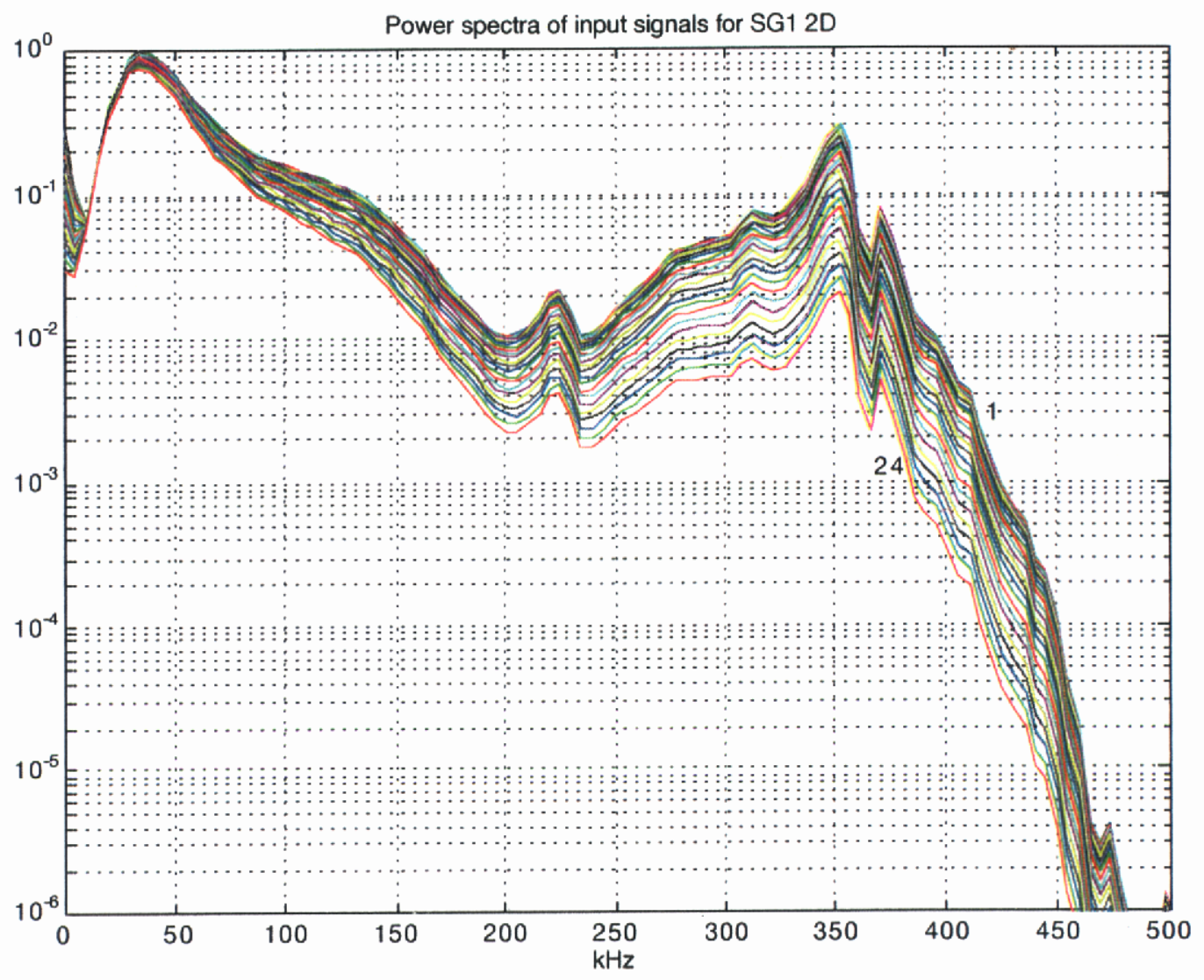

Figure F.5.b The spectra of the SG1 pulse with 2D frequency compensation. The vertical axis is in arbitrary units of amplitude on a log scale (x10 is $10 \mathrm{~dB}$ ), and the horizontal axis is in $\mathrm{kHz}$, from 0 to $500 \mathrm{kHz}$ on a linear scale. 


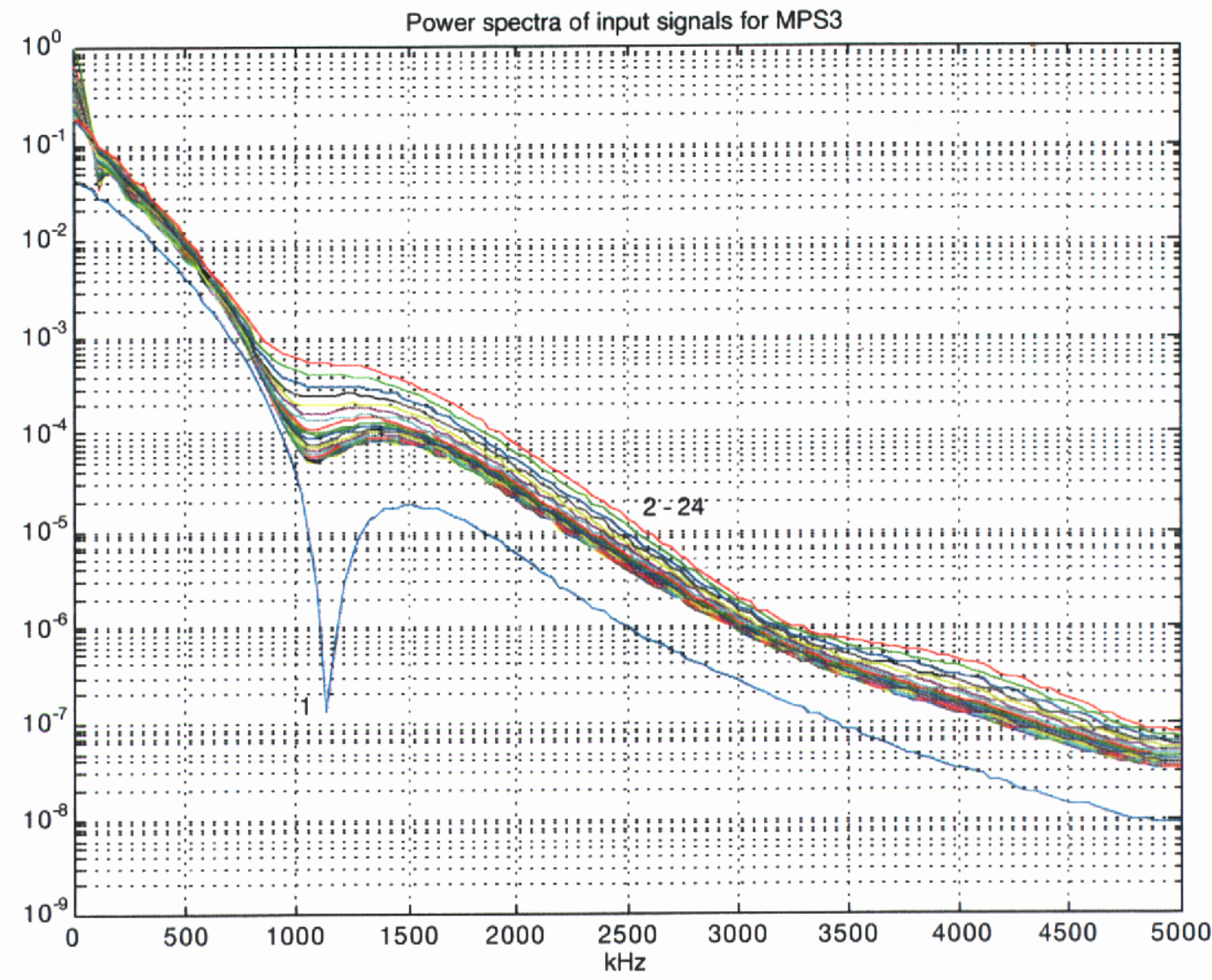

Figure F.7.b The spectra of the MPS3 pulse with no frequency compensation. The vertical axis is in arbitrary units of amplitude on a log scale (x10 is $10 \mathrm{~dB})$, and the horizontal axis is in $\mathrm{kHz}$, from 0 to $5 \mathrm{MHz}$ on a linear scale to emphasize the very high frequency of this pulse. center signal 
University of California/Livermore, CA / $94550 /$ USA

Phone: (510)422-0217

Fax: (510)424-3040

SSSP Strategic Systems Support Program

Law rence Livermore $\mathbf{N}$ atio nal Laboratory

E-mail:sssp@llnl.gov

\title{
Localized Wave Application in Acoustics \\ Test Plan and Objectives for the Lake Travis Experiments with U. Texas Applied Research Laboratory
}

\author{
D. Kent Lewis \\ Strategic Systems Support Program \\ Lawrence Livermore National Laboratory \\ 7000 East Ave, Mail Stop L - 372 \\ Livermore, CA 94550 \\ (510) 422-7959 \\ kent-lewis@llnl.gov
}

Abstract:

This is a test plan for experiments planned with the University of Texas Applied Research Laboratory in Austin. These tests will be performed in March - April 1998 at the UT-ARL facilities. The platforms to be used are the test tank in their headquarters building, and their fixed test barge and test tower in Lake Travis.

Objectives:

The Lake Travis tests are a distance test for the Localized Wave pulse theory. The question of the stability of these pulses when propagated over long distances will be investigated. The other objectives of these experiments are fivefold.

The first is to test digital signal generation and adaptation with an individually addressable array. Signals will be designed for launch from array rings based on the ring's position in the array. Comparison of experimental results to simulations will be made, precorrections made and the new signals launched. If the media, the lake water, shows frequency dependent effects on the pulses, this will also be folded in and corrected for.

The second objective is to make initial tests on the sensitivity of the pulses to the array's source density. The 24 discrete ring elements will be regarded as 24 sources, 12 sources, 6 sources and so on by launching signals from individual rings, pairs of rings, groups of 3 and so on. Comparisons to simulations will be made in all cases.

The third objective is the generation and propagation of LW pulses, both the Modified Pulse Spectrum and Superposed Gaussian beams. Several different pulses have been designed which have different properties. These will be launched and the resulting measurements compared to simulations. In the event of differences, modifications of the beams will be made and new pulses launched.

The fourth objective of the experiments is the comparison of the Localized Wave pulses to conventional transmissions in which all array elements are driven with the same single waveform (e.g., a CW-pulse, FM-sweep, or band-limited impulse, but allowing differently shaded amplitudes across the face of the array). Comparisons will be made of the beam patterns, the on-axis acoustical energy flux normalized by the energy necessary to create them, and the sustainable bandwidth during propagation.

The fifth objective is to refine our understanding of the physics of localized wave generation and design by careful evaluation of the points of agreement and disagreement between experimental measurements and simulations. This contributes to the long term goal of developing a "toolbox" of techniques for designing localized waves for particular applications and array configurations.

Each of these objectives will be accomplished with utmost attention to thoroughness, accuracy, documentation, and safety. 
Test approach:

The test plan is composed of operations with three different acoustic path environments and ranges between source and receiver, each involving four steps. The first acoustic path is a short one ( $20 \mathrm{ft}$ ) entirely within the acoustic test tank at ARL; it will be used primarily for diagnostics and determination of available dynamic range and other operating limits. The second acoustic path is on the Lake Travis Test Station (LTTS) barge, with the source on a test column and the receiver on a second test-column (initially) or suspended from a more distant point on the barge (subsequently). The third acoustic path is from the barge to an existing test tower in Lake Travis, in which the array will be mounted on a test column and the detector mounted on the tower at the standard AKL test distance. The distances available for the barge are nominally $40 \mathrm{ft}$., 50 yards, and 200 yards.

The steps to be performed are 1) calibrate the electronics system by generating and measuring test signals; 2) locate and align the axis of the array-generated beam by timedelay matching between the arrivals of signals from the special corner elements of the projector; 3) calibrate the coupling of the electronics and transducer array with a set of test signals; 4) launch reference and test beams and record the received signals, analyzing for beam patterns (distribution of received energy vs. bearing angle and frequency), and onaxis acoustic energy flux and spectral bandwidth of each. All tests will be carried out as a collaborative effort by LLNL and ARL:UT. Additional details are described in the ARL/UT document "Test Plan for Localized Waves", February 25, 1997.

Organizational Roles:

Conduct tests:

LLNL: Provide Matlab waveforms, test procedure, and test plan, experimental direction and assistance

ARL/UT: Operate equipment to generate waveforms, provide facility test plan

Record data:

LLNL: Provide equipment, computers, recorders as requested by

ARL. Provide personnel to record data.

ARL/UT: Provide baseline equipment and personnel to insure collection

Equipment:

LLNL: Provide any specialized items as requested by ARL

ARL/UT: Provide baseline test equipment, custom transducers, and driver electronics

Data Analysis:

LLNL: Provide detailed data analysis

Test Reports:

ARL/UT: Provide analysis support as requested by LLNL

LLNL: Generate primary test report presenting results and conclusions

ARL/UT: Generate supporting test facility report presenting observations, etc. from ARL point of view

Participant Roles and Responsibilities:

LLNL:

Kent Lewis (primary LLNL test scientist and principal investigator):

1. Decides which waveforms are to be supplied to ARL for generation

2. Interfaces with ARL test conductor to request

a. Waveform generation 
b. Data recording

c. Modifications of test set up

3. Interfaces with ARL test director on key test decisions

4. Insures test objectives are met

David Chambers (participating LLNL scientist):

1. Supplies waveforms to ARL as directed by Kent Lewis

2. Assumes POC role in the absence of the primary LLNL scientest

3. Performs preliminary on-site analysis to evaluate performance of system and to evaluate how well each objective is attained

4. Assists primary LLNL scientist in evaluating experiment progress

5. Assure that data is collected, archived, and properly identified

ARL/UT:

Terry Henderson (ARL test coordinator and POC):

1. Interfaces with LLNL test scientists to insure:

a. Tests are properly conducted

b. Data is properly recorded

c. Criterion is met before proceeding to next stage of testing

d. Test objectives are met

2. Coordinate other ARL test personnel in performance of test

Ken Kruger (ARL Engineer)

1. Assumes ARL POC role in the abscence of the primary ARL/UT scientist

2. Participates in tests by assisting other personnel as required

3. Provides back up confirmation to assure all data is properly collected and all required tests are conducted

Criteria for Proceeding to the next stage of test set up:

Stages are defined as

I. Characterization of transducers and waveform generation (within the well of the barge, maximum distance of $40 \mathrm{ft}$ )

II. Medium distance test (across barge, 40 yards)

III. Long distance test (barge to test tower, 200 yards)

The criterion for proceeding from one stage to the next shall be a consensus

between the LLNL primary test scientist and the ARL engineer that:

1) the database has been successfully filled to achieve test objectives for that stage,

2) it has been demonstrated that the localized wave can be successfully

generated over the distances called for in the present stage

3 ) there is every expectation that the localized wave can be successfully generated over the distances called for in the next stage

\section{Data Collection:}

Data collection is the responsbility of the LLNL and UT/ARL primary scientists. Each scientist will have the entire set of data collected for each stage at the end of the test. Actual implementation of on-site data collection and archiving will be delegated by the consensus of the primary scientists to participating personnel as required during the test.

Data Analysis and Reporting: 
On-site data analysis will be performed by the participating LLNL scientist and the UT/ARL participating engineer under the oversite of the primary scientists. LLNL will be responsible for post-test analysis of localized wave performance and evaluation of the array design for the purpose of generation LWs. UT/ARL will be responsible for general analysis of array and system performance. ARL will also include other analysis and observations of the test as required from their point of view (see Organizational Roles above).

Success criteria:

The tests will be successful if the Localized wave pulses are generated by the individually addressable array and digital driving system, and if they propagate in accordance with our simulation results. Simulations of the performance of candidate Localized Wave pulses at the nominal tests distances were reported in the earlier LLNL report "Simulation of Localized Wave Performance at the Upcoming Lake Travis Experiment", December 5, 1997.

The energy efficiency, or normalized on axis energy, tests will be an additional advantage should they show less energy needed to generate the LW beams. However the true test involved in this experiment is whether the LW pulse will propagate over a significant distance (200 yards) without unexpected degradation, while achieving results superior to conventional sonar wavcforms.

Submitted:

Date:

Approved:

Date: 


\section{Localized Wave Application in Acoustics.}

Proposal 97A190597dkl

D. Kent Lewis, PI Wave Phenomena
Strategic Systems Support Program

0 . Introduction

This is a proposal for research on the Localized Wave for the period June through September, 1997. The cost of this work is $\$ 466.7 \mathrm{k}$.

\subsection{Summary}

The purpose of the present work is to build on our success in the Keyport facility tests. We were able to launch a pair of Localized Wave pulses, the Modified Pulse Spectrum (MPS) and Superimposed Gaussian (SG), and achieve both a times 10 increase in efficiency for the total on axis energy and the maximum on axis pressure, as well as attaining nearly a factor of 10 in total band width, from 8 to 65 $\mathrm{kHz}$. We accomplished all this with a torpedo array which was designed to radiate in a very limited frequency range.

To build on this success, we propose to test the beam at higher power levels, more typical of operational scenarios. We will use, after possible modifications, the present system drivers and electronics for a development array. Our thrust will be to achieve longer distances and higher power levels.

We are taking a system approach in this work. The system we will work with consists of five parts, described below. Even though we have broken the work out into sections, we will need contributions from all the parts to advance out development.

\subsection{Background}

The Localized Wave project has now carried out 5 proof of principle tests to show feasibility. In the last 3 of these tests, a LW pulse was actually launched. Our most recent tests, using an AdCap array head in the Navy's Keyport test facility, showed that the pulse could be launched from an array which was designed for a single frequency. Our preliminary results show a times 10 improvement in signal generating power along with a broad band width which extends from $8 \mathrm{kHz}$ to $65 \mathrm{kHz}$. Side lobes were present, although they were more than $20 \mathrm{~dB}$ down from the main beam, and composed of very high frequency energy in a narrow band width.

\subsection{Concept}

LW pulses are generated using a superposition of linear wave field basis functions. These basis functions are the Focus Wave Mode solutions to the generalized wave equation. The FWM solutions are functions in rho, the off beam axis distance, and ( $\mathrm{z}-\mathrm{ct}$ ) the speed normalized down range distance, and are highly concentrated pulses. As field solutions, like the infinite plane wave for example, the FWMs propagate forever and never change in any way. They are impossible to create physically, in exactly the same way infinite plane waves are impossible to create, needing an infinite amount of energy and an infinitely large aperture.

One recipe for assembling the FWMs into the Localized Wave pulse is a spectrum called the Modified Pulse Spectrum. The MPS gives the complex coefficients of the different FWM components which make up the given LW pulse field. The MPS introduces additional parameters into the beam, other than the FWM number $\mathrm{k}$, and allows one to tailor the pulse for given applications. 
Additionally, other basis functions may be used to generate LW pulses. We have . tested a tailored Gaussian pulse has been tested which exhibits all of the characteristics of a Localized Wave pulse. Efforts are underway to define the relationship between the MPS and superimposed Gaussian basis sets.

The FWM, and therefore any $\mathrm{LW}$ beam, is derivable in terms of the standard set of plane waves, although the expressions become very cumbersome. Creating a specified bundle of field energy is best approached by using building blocks which already have the desired characteristics, and the FWM functions led directly to this new formulation of field variables.

\subsection{Approach}

We take a systems approach to turning the LW phenomenon from a laboratory curiosity into a usable field system. The elements of the system which we identify are 1) Sound Signal Design, 2) Active Systems Design, 3) Measurement Sensitivity, 4) Processing and Identification, and 5) Laboratory and Field Systems Upgrade. These are the crucial parts of any detection or imaging problem, and we must assure that the necessary tools are available when needed.

We envision creating a Localized Wave "toolbox" which can be applied to a wide variety of problems in acoustic and microwave work. The tools we will develop include both software and hardware, all applicable to imaging, location and detection problems. Our goal is a design capability which will allow one to achieve the best possible results for any target in any media with minimum cost.

\section{Source Signal Design}

The most fundamental part of our system is the source signal set. These are used in both the creation and detection phases of the technique. At the same time, the LW source signals are not the only possible signals we could use. We will continue to investigate the generalized family of low diffraction pulsed beams.

Part of our work will also be to determine what changes can be made to these signals without markedly decreasing the efficiency of the pulse. We will find ways to design LW fields for communication and encryption. We will examine ways to include information in the pulses for communication.

\subsection{Localized Wave Transform.}

We believe that it is possible to form a $\mathrm{LW}$ transform of any arbitrary wave field in the same way that a K-omega transform is formed presently. We know that an orthogonality relationship exists between FWMs in the on axis direction and it is reasonable that a similar expression for non-aligned FWMs exists. Finding such an expression will allow an angular as well as temporal design tool to be built, and any arbitrary pulse field to be constructed by finding its $\mathrm{k}(\mathrm{rho}), \mathrm{k}(\mathrm{z})$ and temporal frequency components.

\subsection{Generalized Low Diffraction Theory}

As an investment in the future, we will study characteristics of existing low diffraction beams and generalize the theory to identify other members of this most interesting family. Our goal is to identify higher efficiency basis sets for generalized LW pulses, which we believe exist, and to determine which members of the set are best used in different problems.

2. Active Systems Design

2.1 Active Array Design

- determine necessary characteristics needed for efficient generation 
- characterize and modify electronics presently used for new drivers

- design optimize drivers for full up LW array

\subsection{Field Trials}

- test generation on multiple element arrays

- test existing electronics modified for sufficient band width

3. Measurement Sensitivity

3.1 Array and Environment Modeling

- acquire or build models of systems of interest

\subsection{Measurement System Simulation}

- acquire or build models of measurement systems in use

- design new measurement systems if necessary for extended band width

\subsection{Noise Simulator}

- acquire or build models of noise in interesting environments

- design spatially dependent noise sources

4. Processing and Identification

\subsection{Optimal Filter Design for LW Fields}

- find optimal filter technique applied to LW

\subsection{Sensitivity Determination}

- determine sensitivity of processor schemes as applied to broad band width pulse

5. Laboratory and Field Systems Upgrade

Our experience in Keyport pointed up shortfalls in our own capability to prepare for field trials. Our computer systems are antiquated, and we have at present no backup experimental computers. We will correct this flaw, and build a robust and easily replaceable system from commonly available components.

We spent too much time simply learning the characteristics of the arrays and sensing elements which we used. This could have been avoided by outfitting our newly acquired large tank facility for some initial testing. We will equip our own laboratory systems to avoid this problem in the future.

\subsection{Field system upgrade}

- upgrade command computer to readily available components

- maintain a backup of the entire system to avoid down-time

5.2 Testbed Upgrade

- outfit our laboratory systems for initial in-house testing 
6.0 Cost breakout for $L W$ project.

6.1 Expenses: Averaged for each team member per FTE

Average Salary and Overhead:220k

Includes all laboratory, departmental, security and safety overhead charges

Average Equipment: $25 \mathrm{k}$

Office computers and software, office space charge

Conferences $8 \mathrm{k}$

One to two conferences per year for peer review of unclassified work Travel $\underline{27 \mathrm{k}}$

Based on past work costs and expectation of off site work

Total cost per FTE $\quad 280 \mathrm{k}$

7.1.1 Upgrade costs entered as FTE expense at equivalent amount.

6.2 Projected cost of work June through September, 1997

June through September 1997

Research and development - $326.7 \mathrm{k}$

Field system upgrade $\quad 70.0 \mathrm{k}$

includes $23.3 \mathrm{k}$ manpower costs

Testbed upgrade

$70.0 \mathrm{k}$

includes $23.3 \mathrm{k}$ manpower costs

Total cost for period $\quad 466.7 \mathrm{k}$

Contact:

D. Kent Lewis, PI Wave Phenomena

Strategic Systems Support Program

Lawrence Livermore National Laboratory

7000 East Ave, MailCode L-372

Livermore, CA 94551-0808

Tel: $\quad(1-510) 4227959$

Fax: (1-510) 4243040

email: kent-lewis@1lnl.gov 